\title{
Development of an indentation method for material surface mechanical properties measurement
}

\author{
Ziheng Yao \\ West Virginia University
}

Follow this and additional works at: https://researchrepository.wvu.edu/etd

\section{Recommended Citation}

Yao, Ziheng, "Development of an indentation method for material surface mechanical properties measurement" (2005). Graduate Theses, Dissertations, and Problem Reports. 2654.

https://researchrepository.wvu.edu/etd/2654

This Dissertation is protected by copyright and/or related rights. It has been brought to you by the The Research Repository @ WVU with permission from the rights-holder(s). You are free to use this Dissertation in any way that is permitted by the copyright and related rights legislation that applies to your use. For other uses you must obtain permission from the rights-holder(s) directly, unless additional rights are indicated by a Creative Commons license in the record and/ or on the work itself. This Dissertation has been accepted for inclusion in WVU Graduate Theses, Dissertations, and Problem Reports collection by an authorized administrator of The Research Repository @ WVU.

For more information, please contact researchrepository@mail.wvu.edu. 


\title{
Development of an Indentation Method for Material Surface Mechanical Properties Measurement
}

\author{
Ziheng Yao
}

Dissertation submitted to the College of Engineering and Mineral Resources at West Virginia University in partial fulfillment of the requirements for the degree of

\author{
Doctor of Philosophy \\ in \\ Mechanical Engineering \\ Bruce Kang, Ph.D., Chair \\ Ever J. Barbero, Ph.D., Co-Chair \\ Xingbo Liu, Ph.D. \\ Julio F. Davalos, Ph.D. \\ Robert C. Creese, Ph.D. \\ Department of Mechanical and Aerospace Engineering \\ Morgantown, West Virginia \\ 2005
}

Keywords: Indentation, Mechanical property, Finite Element, Thin Film Copyright ( 2005 Ziheng Yao 


\section{Abstract \\ Development of an Indentation Method for Material Surface Mechanical Properties Measurement}

\section{Ziheng Yao}

With the utilizing structural materials with small size dimensions (such as MEMS devices), there are growing need to characterize the mechanical properties of the small volumes of materials. Tensile test is not possible to be applied to these small volumes. Indentation technique has emerged as a cost-effective, convenient and non-destructive method to solve this problem. It is also a promising means of obtaining mechanical properties for thin films, which were widely used in various industries nowadays.

In this work, finite element simulations of spherical indentation on various material models were performed to develop a series of methodology, by which the material surface mechanical properties are to be determined through indentation test. Finite element mesh and modeling were first verified by both Hertz analytical solution and experiment results of surface displacement fields from combined Moiré Interferometry and Twyman-Green Interferometry. Method to calculate elastic properties through initial unloading indentation stiffness obtained in load depth curve is verified. Special attention was put to the relationship between the surface displacement fields (U (in-plane radial displacement) and W (out-of-plane displacement)) and material mechanical properties. It is discovered that each point on the surface experienced same equivalent stress strain history governed by input uniaxial stress strain curve. Elasticplastic boundary corresponds to characteristic point in the $\mathrm{W}$ field variation, which indicated an experimental method to capture the E-P boundary. The yield strength could be calculated from Hooke's law by strain fields derived from displacements using kinematic equations. The strain-hardening exponent is to be obtained by modified Meyer's law or Tabor's relation. Hence, the uniaxial stress strain relation of the material could be reestablished using indentation method.

Then the micro spherical indentations on thin film - substrate systems were simulated. The influence of substrate on the load depth curve, surface deformation was studied for both soft film on hard substrate and hard film on soft substrate cases. Then different thickness of thin film was simulated and it is concluded that the effect of substrate is negligible when the indentation depth is less than $10-20 \%$ of film thickness. 


\section{Acknowledgements}

At first, I wish to thank my research advisor and committee chair, Dr. Bruce Kang, for his intellectual support and enthusiasm on the whole process of this research project and dissertation writing, which made this dissertation possible. I thank Dr. Barbero for serving on my dissertation committee co-chair. I genuinely appreciate for his continuous encouragement. Thank all the other committee members, Dr. Xingbo Liu, Dr. Davalos and Dr. Robert Creese, for their precious suggestions and persistent support.

Colleagues and friends in the research group offered many discussions that were helpful for this work. I would like to thank Chuanyu Feng for all his help and discussions during the course of the research.

Special appreciation for Dr. F.M. Haggag, president and chief engineer from Advanced Technology Corp., who provided valuable and substantial help.

I am grateful to my parents for their support and encouragement. At last, this dissertation is dedicated to my wife, Chaohui and my beloved sons, Kevin and Steven. They are my indispensable impetus to move forward. 


\section{Table of Contents}

$\begin{array}{lr}\text { Abstract } & \text { ii } \\ \text { Acknowledgements } & \text { iii } \\ \text { Table of Contents } & \text { iv } \\ \text { List of Figures } & \text { viii } \\ \text { List of Tables } & \text { xi } \\ \text { List of Symbols } & \text { xii }\end{array}$

\section{Chapter 1. Introduction}

1.1 Introduction to hardness test 1

1.2 The contemporary application of indentation technique 5

1.3 Two theoretical branches of current indentation research 9

$\begin{array}{ll}1.4 \text { Objectives } & 12\end{array}$

\section{Chapter 2. Literature Review}

2.1 Analytical solution development of indentation $\quad 15$

2.2 Determination of Young's modulus by load-depth sensing indentation 18

2.3 Correlation between yield strength and hardness 23

2.4 Methodology to extract stress-strain curve from indentation 24

2.5 Surface deformation analysis 28

2.6 Thin film - substrate system 31

\section{Chapter 3. Finite element modeling}

3.1 Introduction to ABAQUS software package 34

3.2 Nonlinear problem analysis in ABAQUS 34

3.3 Material mechanical behavior 36 
3.3.1 Elasticity and plasticity 36

3.3.2 Engineering and true stress-strain relationship 36

3.3.3 Mathematical models to describe stress-strain curve 38

3.4 Finite element modeling of indentation $\quad 40$

3.4.1 Theoretical background 40

3.4.2 Material property input $\quad 42$

3.4.2 Mesh design 43

3.4.4 Boundary conditions and applying load 46

3.4.5 Contact interaction 46

\section{Chapter 4. Factors to influence results}

4.1 Element selection: 'full integration' or 'reduced integration' 48

4.2 Two methods of applying load: 'load control' and 'displacement control' 48

4.3 Boundary condition effect 50

4.4 Coefficient of friction effect 53

4.5 Indenter size effect $\quad 56$

\section{Chapter 5. Verification of finite element model}

5.1 Verification by comparison with elastic analytical results 59

5.1.1 Hertz theory of elastic indentation 59

5.1.1.1 Surface stress and displacement distribution $\quad 60$

5.1.1.2 Load depth (P-hs) relationship 61

5.1.2 Elastic finite element model 62

5.1.3 Comparison between FEM results and analytical solution 63

$\begin{array}{ll}5.2 \text { Experimental verification } & 67\end{array}$

\section{Chapter 6. Data analysis}

6.1 Simulation matrix: material library selection $\quad 70$

6.2 Tabor's stress strain relation $\quad 71$

6.3 Load depth curve 79 
6.4 Surface displacement and strain fields $\quad 85$

6.4.1 Surface displacement fields $\quad 85$

6.4.1.1 Single node tracing for loading process 85

6.4.1.2 Surface displacement fields at certain loading status $\quad 86$

6.4.2 Surface strain fields $\quad 89$

6.4.2.1 Strain components $\quad 89$

6.4.2.2 Total strain $\quad 92$

6.5 Stress strain relation $\quad 94$

6.5.1 Elastic zone and plastic zone $\quad 94$

6.5.2 Stress strain relation (single node tracing for loading process) 95

6.5.3 Stress strain relation (surface contour at certain loading status) 97

6.6 Elastic rebound during unloading and cyclic reloading 102

$\begin{array}{ll}\text { 6.6.1 Elastic rebound during unloading } & 102\end{array}$

6.6.2 Cyclic reloading 106

6.7 Strain hardening and Meyer's law 108

$\begin{array}{ll}6.8 \text { Conclusions and discussions } & 114\end{array}$

6.8.1 Two methods to get Young's modulus, E value 114

6.8.2 Yield stress, $\sigma_{\mathrm{y}}$ determination $\quad 115$

6.8.3 Strain hardening exponent determination $\quad 116$

$\begin{array}{ll}\text { 6.8.4 Discussions } & 116\end{array}$

\section{Chapter 7. Thin film - substrate system}

$\begin{array}{ll}7.1 \text { Introduction } & 118\end{array}$

7.2 Soft film on hard substrate and hard film on soft substrate 119

$\begin{array}{ll}\text { 7.2.1 Load depth curve and Young's modulus calculation } & 119\end{array}$

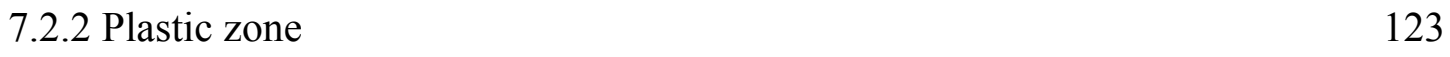

$\begin{array}{ll}\text { 7.2.3 Tabor's relation } & 125\end{array}$

7.2.4 Surface deformation 128

$\begin{array}{ll}7.3 \text { Thin film thickness effect } & 130\end{array}$ 
Chapter 8. Conclusions and discussions

$\begin{array}{ll}\text { Bibliography } & 135\end{array}$

Curriculum Vitae $\quad 142$ 


\section{LIST OF FIGURES}

Figure 1.1 (a) Scheme of Vickers hardness test [43]. 2

Figure 1.1 (b) Scheme of Vickers impression [43]. 2

Figure 1.2 Schematic diagram of Rockwell hardness test [43]. 3

Figure 1.3 Schematic diagram of Brinell hardness test [43]. 4

Figure 1.4 Degradation of material properties of a GTD 111 DS First Stage Blade $\begin{array}{ll}\text { after } 49,000 \text { hours of service. } & 8\end{array}$

Figure 1.5 Spherical indent in 1015 steel (20 $\mu \mathrm{m}$ grain size $)$ obtained at a force of $2 \mathrm{~N}$ using a $254 \mu \mathrm{m}$ (0.010 in) diameter indenter. 13

Figure 2.1 Johnson's elastic-plastic model [2,3]. 15

Figure 2.2 Schematic drawing of load displacement curve. 19

Figure 2.3 Different regimes of plastic flow at the tip of a sharp indenter [19]. 25

Figure 2.4 Schematic drawing of piling-up and sinking in of material around spherical indents.

Figure 2.5 Dependence of the surface deformation parameter $\mathrm{c}^{2}-1$ for spherical indentation on the strain hardening exponent $\mathrm{n}[18] . \quad 30$

Figure 3.1 Engineering and true stress strain curve (Aluminum $6061 \mathrm{~T} 6$ ). 37

Figure 3.2 (a) Mesh design in the entire model. $\quad 44$

Figure 3.2 (b) Magnified view of mesh design under the sphere indenter. 45

Figure 4.1 Load-depth curve comparisons for different loading methods. 50

Figure 4.2 (a) Scheme of (A) boundary condition setup (roller bottom, roller side). 51

Figure 4.2 (b) Scheme of (B) boundary condition setup (fixed bottom, free side). $\quad 51$

Figure 4.3 (a) Load-depth curve for different boundary conditions. 52

Figure 4.3 (b) Surface W field contour for different boundary conditions. 52

Figure 4.4 (a) Load-depth curve comparisons for different coefficient of friction. $\quad 54$

Figure 4.4 (b) U2 distributions for the same material but different friction property. $\quad 55$

Figure 4.5 Normalized surface displacement distribution for different indenter size. 56

Figure 4.6 Normalized surface von Mises distribution for different indenter size. $\quad 57$

Figure 4.7 Normalized load depth curve for different indenter size. 58

Figure 5.1 von Mises stress contour plot beneath the rigid spherical indenter. 63

Figure 5.2 (a) W $\left(\mathrm{u}_{\mathrm{z}}\right)$ field comparison between FEM and analytical results. $\quad 64$

Figure $5.2(\mathrm{~b}) \sigma_{\mathrm{z}}$ comparison between FEM and analytical results. $\quad 64$

Figure $5.3(\mathrm{a}) \mathrm{U}\left(\mathrm{u}_{\mathrm{r}}\right)$ field comparison between FEM and analytical results. 65

Figure 5.3 (b) $\sigma_{\mathrm{r}}$ comparison between FEM and analytical results. 65

Figure 5.4 Load depth curve comparison between FEM and analytical results. $\quad 66$

Figure 5.5 (a) In-plane U field fringe pattern $(0.833 \mu \mathrm{m} /$ fringe $)$.

Figure 5.5 (b) Out-of-plane W field fringe pattern $(0.3164 \mu \mathrm{m} /$ fringe $)$.

Figure 5.6 (a) Residual out-of-plane W field comparison. $\quad 68$

Figure 5.6 (b) Residual in-plane U field comparison $\quad 69$ 
Figure 6.2.1 Tabor's stress strain relation

(a) Material set $\mathrm{E}=69 \mathrm{GPa}, \sigma_{\mathrm{y}}=275 \mathrm{MPa}, \mathrm{n}=0.09,0.18,0.27,0.36$ respectively. $\quad 72$

(b) Material set $\mathrm{E}=69 \mathrm{GPa}, \sigma_{\mathrm{y}}=500 \mathrm{MPa}, \mathrm{n}=0.09,0.18,0.27,0.36$ respectively. $\quad 73$

(c) Material set $\mathrm{E}=200 \mathrm{GPa}, \sigma_{\mathrm{y}}=242 \mathrm{MPa}, \mathrm{n}=0.1,0.2,0.3$ respectively. $\quad 73$

(d) Material set $\mathrm{E}=200 \mathrm{GPa}, \sigma_{\mathrm{y}}=500 \mathrm{MPa}, \mathrm{n}=0.1,0.2,0.3$ respectively. $\quad 74$

(e) Material set $\mathrm{E}=200 \mathrm{GPa}, \sigma_{\mathrm{y}}=750 \mathrm{MPa}, \mathrm{n}=0.1,0.2,0.3$ respectively. $\quad 74$

(f) Material set $\mathrm{E}=26.2 \mathrm{GPa}, \sigma_{\mathrm{y}}=22.5 \mathrm{MPa}, \mathrm{n}=0.026,0.1,0.2,0.3$ respectively. $\quad 75$

Figure 6.2.2 Finite element graph of plastic zone expansion during ball indentation

(a) Elastic regime $\quad 76$

(b) Elastic-plastic regime $\quad 76$

(c) Fully plastic regime $\quad 77$

$\begin{array}{ll}\text { Figure 6.2.3 } p_{m} / \sigma \text { fluctuation. } & 78\end{array}$

Figure 6.3.1 Load depth curves

(a) Material set $\mathrm{E}=69 \mathrm{GPa}, \sigma_{\mathrm{y}}=275 \mathrm{MPa}, \mathrm{n}=0.09,0.18,0.27,0.36$ respectively. $\quad 79$

(b) Material set $\mathrm{E}=69 \mathrm{GPa}, \sigma_{\mathrm{y}}=500 \mathrm{MPa}, \mathrm{n}=0.09,0.18,0.27,0.36$ respectively. $\quad 80$

(c) Material set $\mathrm{E}=200 \mathrm{GPa}, \sigma_{\mathrm{y}}=242 \mathrm{MPa}, \mathrm{n}=0.1,0.2,0.3$ respectively. $\quad 80$

(d) Material set $\mathrm{E}=200 \mathrm{GPa}, \sigma_{\mathrm{y}}=500 \mathrm{MPa}, \mathrm{n}=0.1,0.2,0.3$ respectively. $\quad 81$

(e) Material set $\mathrm{E}=200 \mathrm{GPa}, \sigma_{\mathrm{y}}=750 \mathrm{MPa}, \mathrm{n}=0.1,0.2,0.3$ respectively. $\quad 81$

(f) Material set $\mathrm{E}=26.2 \mathrm{GPa}, \sigma_{\mathrm{y}}=22.5 \mathrm{MPa}, \mathrm{n}=0.026,0.1,0.2,0.3$ respectively. $\quad 82$

Figure 6.4.1 Two nodes W displacement variation vs. indentation depth. 85

Figure 6.4.2 $\mathrm{U}$ and $\mathrm{W}$ contour distribution at maximum loading and total unloading status

(a) $\mathrm{n}=0.09$. 86

(b) $\mathrm{n}=0.18$.

(c) $n=0.27$.

(d) $n=0.36 . \quad 88$

Figure 6.4.3 Strain components E11 and E33 distribution at maximum loading, where $E 11=\varepsilon_{r}, E 33=\varepsilon_{\theta} . \quad 90$

Figure 6.4.4 Strain components E22distribution at maximum loading, where $E 22=\varepsilon_{z} . \quad 91$

Figure 6.4.5 Total strain distribution comparisons. 93

Figure 6.5.1 Elastic and plastic zone at maximum loading $(320 \mu \mathrm{m})$

(a) For piling up material ( $\left.\mathrm{E}=69 \mathrm{GPa}, \sigma_{\mathrm{y}}=275 \mathrm{MPa}, \mathrm{n}=0.09\right)$. 94

(b) For sinking in material ( $\left.\mathrm{E}=69 \mathrm{GPa}, \sigma_{\mathrm{y}}=275 \mathrm{MPa}, \mathrm{n}=0.36\right)$. 95

Figure 6.5.2 Two nodes von Mises stress tracing for loading process. 96

Figure 6.5.3 Total strain vs. von Mises stress by tracing single node for loading $\begin{array}{ll}\text { process. } & 97\end{array}$

Figure 6.5.4 Schematic drawing of surface state of stress for piling up material. 99

Figure 6.5.5 Von Mises stress distribution at both maximum loading and unloading $\begin{array}{ll}\text { status. } & 100\end{array}$

Figure 6.5.6 Stress strain curve obtained from surface von Mises stress and total 101 strain fields.

Figure 6.6.1 Unloading W field comparison between FEM and analytical results

(a) Piling up material, $\mathrm{n}=0.09$. 
(b) Sinking in material, $\mathrm{n}=0.36$.

Figure 6.6.2 Derivative of $\mathrm{W}$ comparison between FEM and analytical results

(a) Piling up material, $\mathrm{n}=0.09$.

105

(b) Sinking in material, $n=0.36$.

105

Figure 6.6.3 Load depth curve comparison: direct loading and cyclic loading. $\quad 107$

Figure 6.7.1 $\log (\mathrm{a})$ vs. $\log (\mathrm{P})$ plot

(a) Material set $\mathrm{E}=69 \mathrm{GPa}, \sigma_{\mathrm{y}}=275 \mathrm{MPa}, \mathrm{n}=0.09,0.18,0.27,0.36$ respectively.

(b) Material set $\mathrm{E}=69 \mathrm{GPa}, \sigma_{\mathrm{y}}=500 \mathrm{MPa}, \mathrm{n}=0.09,0.18,0.27,0.36$ respectively.

109

(c) Material set $\mathrm{E}=200 \mathrm{GPa}, \sigma_{\mathrm{y}}=242 \mathrm{MPa}, \mathrm{n}=0.1,0.2,0.3$ respectively.

(d) Material set $\mathrm{E}=200 \mathrm{GPa}, \sigma_{\mathrm{y}}=500 \mathrm{MPa}, \mathrm{n}=0.1,0.2,0.3$ respectively.

(e) Material set $\mathrm{E}=200 \mathrm{GPa}, \sigma_{\mathrm{y}}=750 \mathrm{MPa}, \mathrm{n}=0.1,0.2,0.3$ respectively.

(f) Material set $\mathrm{E}=26.2 \mathrm{GPa}, \sigma_{\mathrm{y}}=22.5 \mathrm{MPa}, \mathrm{n}=0.026,0.1,0.2,0.3$ respectively.

Figure 6.7.2 n vs. $\mathrm{f}(\mathrm{n})$ plot for 21 material models

Figure 7.1 Mesh design for thin film substrate system simulations.

Figure 7.2 (a) Load depth curve for bulk Si and thin film Si on Al substrate. 120

Figure 7.2 (b) Load depth curve for bulk Al and thin film Al on Si substrate. $\quad 120$

Figure 7.3 Parameter $\alpha$ as a function of normalized punch size. 122

Figure 7.4 (a) Computed plastic regions for bulk aluminum. 123

Figure 7.4 (b) Computed plastic regions for bulk silicon. $\quad 124$

Figure 7.5 (a) Computed plastic zone of soft film (Al) on hard substrate (Si). 124

Figure 7.5 (b) Computed plastic zone of hard film (Si) on soft substrate (Al). 125

Figure 7.6 (a) Tabor's relation comparison for thin film Al and bulk Al. 126

Figure 7.6 (b) Tabor's relation comparison for thin film Si and bulk Si. 127

Figure 7.7 (a) W field distribution: comparison between bulk Al and thin film Al. 129

Figure 7.7 (b) W field distribution: comparison between bulk Si and thin film Si. $\quad 129$

Figure 7.8 (a) Load depth curve for different thickness of aluminum thin film. $\quad 130$

Figure 7.8 (b) W field distribution: comparison for different film thickness. 131

Figure 7.9 Load depth curve for different thickness of silicon thin film. 131 


\section{LIST OF TABLES}

Table 6.1 Simulation matrix: material library selection.

Table 6.2 Using indentation stiffness to calculate Young's modulus.

Table 7.1 Material properties used for the finite element simulations for thin film substrate system

Table 7.2 Using indentation stiffness to calculate elastic modulus for bulk materials.

Table 7.3 Using indentation stiffness to calculate elastic modulus for thin film materials. 


\section{LIST OF SYMBOLS}

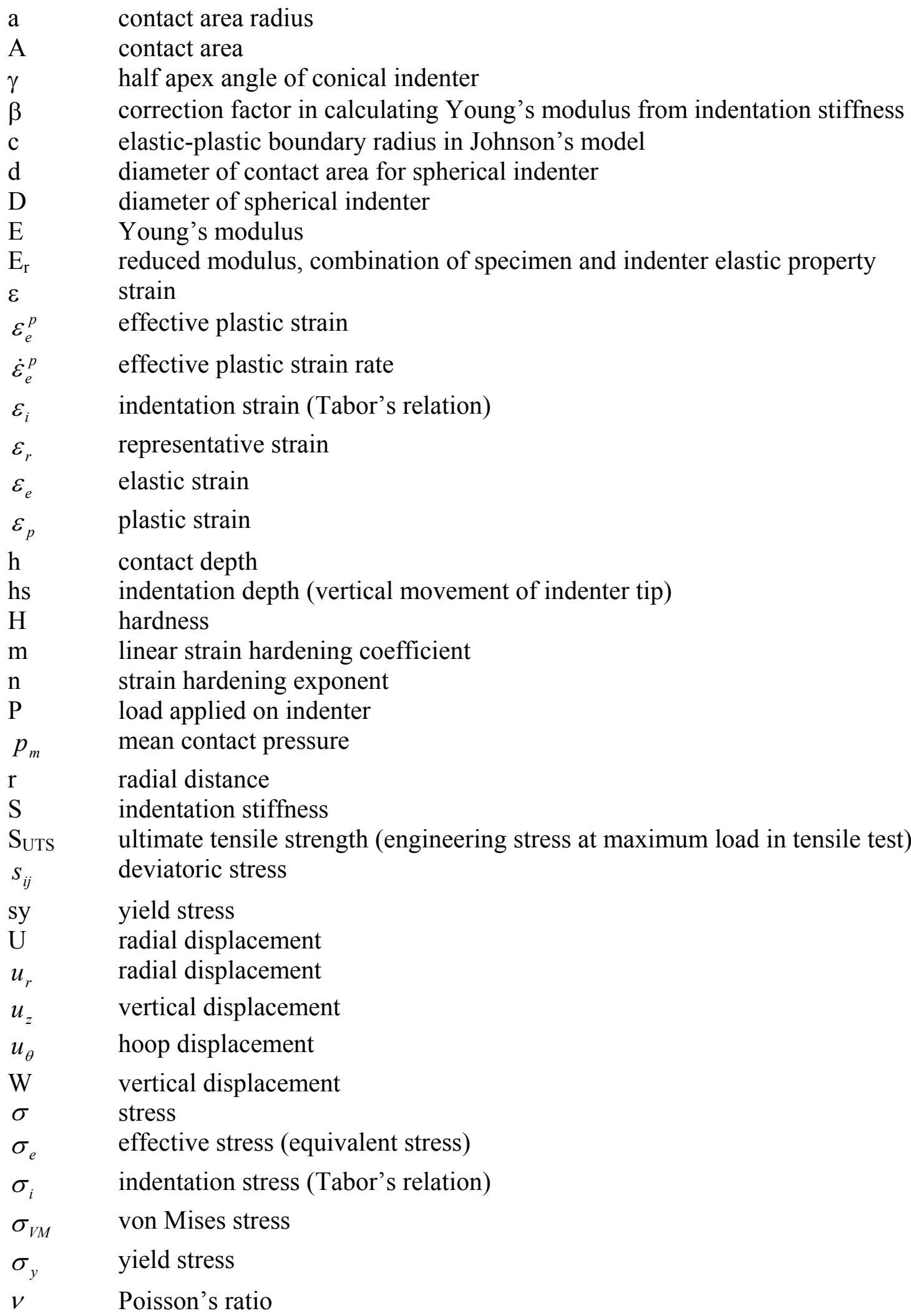


$\delta_{i j} \quad$ Kronecker Delta 


\section{Chapter 1}

\section{Introduction}

\subsection{Introduction to hardness test}

Indentation tests, in many cases referred to as hardness tests, have for a long time been a standard method for material characterization. The hardness test consists of loading an indenter made of diamond or any other hard material (e.g., Tungsten Carbide) and pressing it into the surface of a softer material to be examined. The further into the material the indenter sinks (for a given load), the softer the material is and the lower its yield strength. Hardness is not an intrinsic material property dictated by precise definitions in terms of fundamental units of mass, length and time. A hardness property value is the result of a defined measurement procedure.

Hardness tests are the most commonly used non-destructive testing procedures in the metal industry and in research because they provide an easy, inexpensive and reliable method of evaluating basic properties of developed or new materials. The hardness test indenter is so small that it scarcely damages the bulk material; therefore, it can be used for routine batch tests on small samples of materials to ascertain that they are up to specifications on yield without damaging them.

The usual method to achieve a hardness value is to measure the depth or area of an indentation left by an indenter of a specific shape, with a specific force applied for a specific time. There are three principal standard test methods for expressing the relationship between hardness and the size of the impression, these being Vickers, Rockwell and Brinell.

1) Vickers hardness test 
The Vickers indenter is a square based pyramid with an angle of 136 degrees between the faces and a ratio of diagonals of 1:1 (as shown in Figure 1.1 (a)).

The Vickers hardness number is one of the most widely used measures of hardness in engineering and science. The Vickers diamond hardness, VDH, is calculated using the indenter load and the actual surface area of the impression. The resulting quantity is usually expressed in $\mathrm{kgf} / \mathrm{mm}^{2}$.

$\mathrm{F}=$ Load in $\mathrm{kgf}$

$\mathrm{d}=$ Arithmetic mean of the two diagonals, $\mathrm{d} 1$ and $\mathrm{d} 2 \mathrm{in} \mathrm{mm}$ (Figure 1.1 (b))

$\mathrm{HV}=\mathrm{Vickers}$ hardness

$H N=\frac{2 F \sin \frac{136^{\circ}}{2}}{d^{2}} \quad H=1.854 \frac{F}{d^{2}}$ approximately
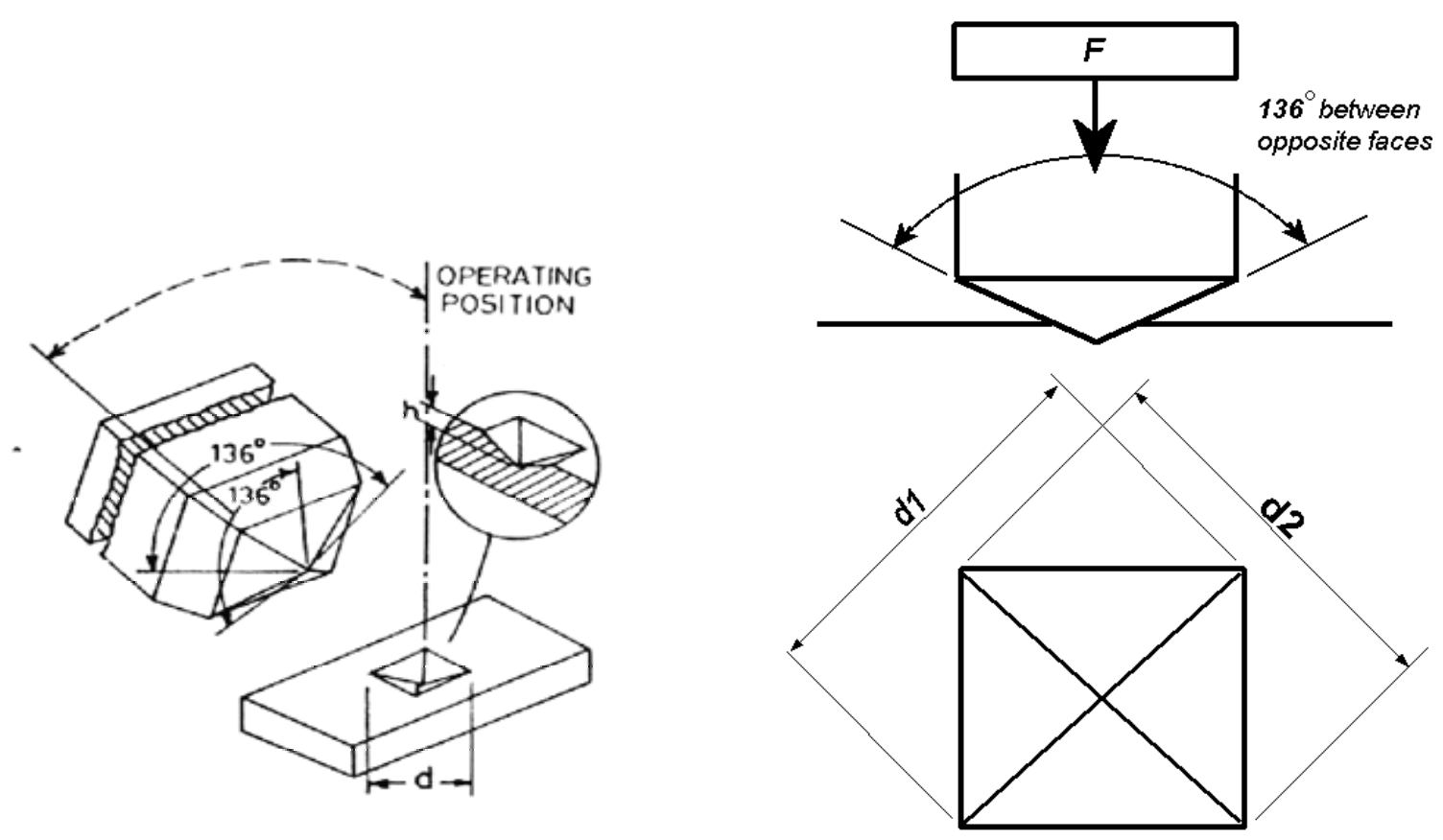

Figure1.1 (a) Scheme of Vickers hardness test [43]

(b) Scheme of Vickers impression [43] 
2) Rockwell hardness test

The Rockwell hardness test method consists of indenting the test material with a diamond cone or hardened steel ball indenter. The indenter is forced into the test material under a preliminary minor load $F 0$ usually $10 \mathrm{kgf}$. When equilibrium has been reached, an indicating device, which follows the movements of the indenter and so responds to changes in depth of penetration of the indenter, is set to a datum position. While the preliminary minor load is still applied an additional major load is applied with resulting increase in penetration. When equilibrium has again been reach, the additional major load is removed but the preliminary minor load is still maintained. Removal of the additional major load allows a partial recovery, so reducing the depth of penetration. The permanent increase in depth of penetration, resulting from the application and removal of the additional major load is used to calculate the Rockwell hardness number.

$$
\mathrm{HR}=E-e
$$

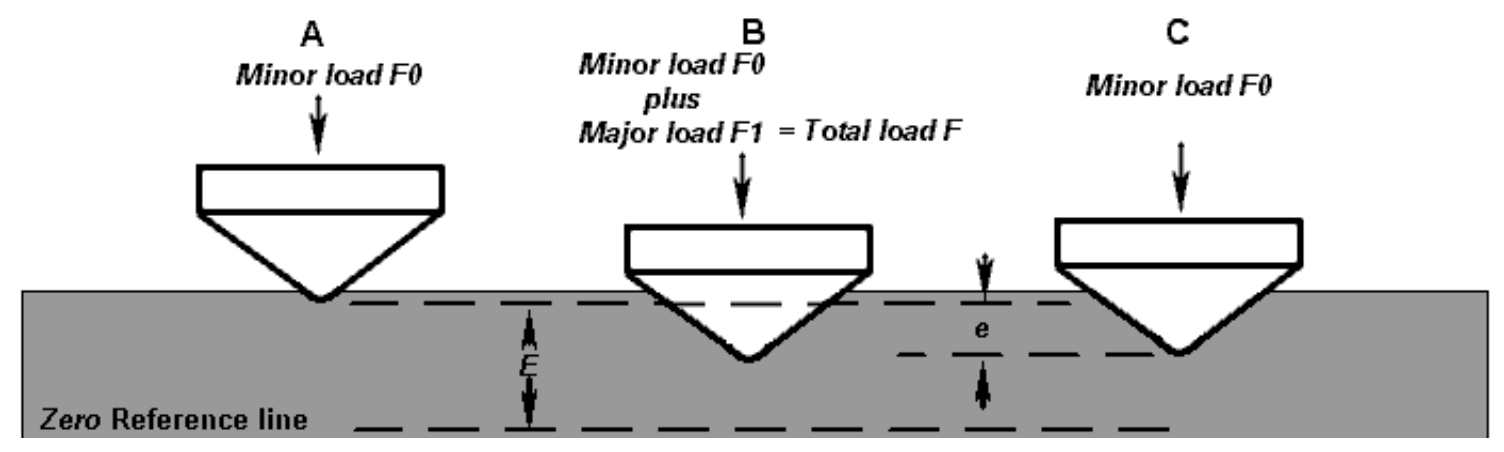

Figure 1.2 Schematic diagram of Rockwell hardness test [43].

Rockwell hardness test scales from A to V, classified by indenter shape and size.

Details could be found in reference [43].

3) Brinell hardness and Meyer hardness 
The Brinell hardness test method consists of indenting the test material with a 10 $\mathrm{mm}$ diameter hardened steel or carbide ball subjected to a load of $3000 \mathrm{~kg}$. For softer materials the load can be reduced to $1500 \mathrm{~kg}$ or $500 \mathrm{~kg}$ to avoid excessive indentation. The full load is normally applied for 10 to 15 seconds in the case of iron and steel and for at least 30 seconds in the case of other metals. The diameter of the indentation left in the test material is measured with a low powered microscope. The Brinell harness number is calculated by dividing the load applied by the surface area of the indentation.

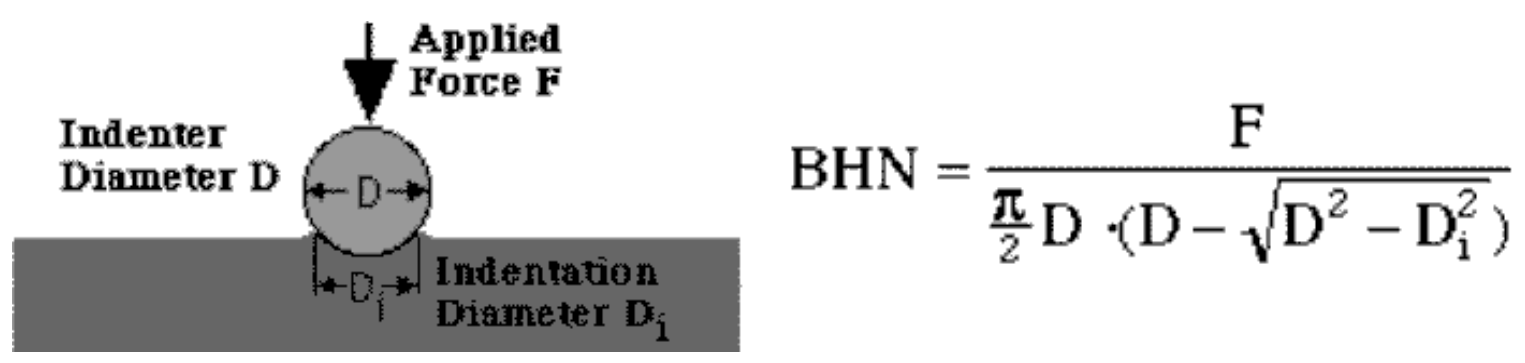

Figure 1.3 Schematic diagram of Brinell hardness test [43].

The Meyer hardness is similar to the Brinell hardness except that the projected area of contact rather than the actual curved surface area is used to determine the hardness. In this case, the hardness number is equivalent to the mean contact pressure between the indenter and the surface of the specimen. The mean contact pressure is a quantity of considerable physical significance. The Meyer hardness is given by: $H=p_{m}=\frac{P}{A}=\frac{P}{\pi a^{2}}$

$\mathrm{A}$ is the projected contact area and $\mathrm{a}$ is the real contact radius during indentation. 


\subsection{The contemporary application of indentation technique}

Although well known for approximately 100 years, classical hardness test has gained new popularity during the last decades.

The advantage of the indentation test, in comparison with a uniaxial tensile test, is of course the relative simplicity of the experimental setup. On the other hand, an obvious drawback is the very complicated mechanical problem arising owing to inelastic and/or inhomogeneous deformation in the indented materials. Therefore, until recently the interpretation of indentation tests have relied heavily on semi-empirical formulae. The work by Tabor [39] (1951) is perhaps the best example of this, with no or little theoretical foundation. With the advent of modern computers and advanced numerical methods, however, the understanding of the mechanics involved during ball indentation (Hill et al., 1989 [56]; Kral et al., 1993 [53]; Larsson, 1994 [40]), cone indentation (Laursen and Simo, 1992 [52]) and Vickers Indentation (Giannakopoulos and Suresh, 1994 [19]), has increased rapidly in recent years.

These new interests in the mechanical behavior of indentation testing are to a large extent a result of the increased use of new materials. These materials are notoriously difficult to characterize through uniaxial or other standard tests, which in many cases make indentation testing the only possible alternative for determining their mechanical properties.

The traits of small size and non-destructive test capability make the indentation technique superior to the tension test. For very small volumes of material, the uniaxial test is inapplicable. Furthermore, the structural materials may not be removed to do the tension test in most cases, for instance, the materials used in the electronic solders or 
engineering welds. Indentation technique can evaluate the material properties while keeping the structural integrity.

Sharp indentation tests also serve to initiate and control fracture in brittle materials. Much effort has been devoted to fracture toughness determination. Other applications that refer to, or use indirectly, the Vickers hardness include abrasive wear problem, armor plating, machining, etc. Most tests are of static type, however, several dynamic tests are also performed.

Another main application area of indentation technique lies in the thin film substrate systems research. Thin film coatings have been widely used in various industries in recent decades. The coated products have become crucial to machine parts performance and durability, multiplying the life span of some parts by 10 to 20 times.

For instance, ceramic thermal barrier coatings (TBCs) have been widely used in gas turbine applications over roughly the past two decades due to their ability to provide heat and corrosion resistance. They are used to insulate and protect such components as combustors, vanes and blades in aircraft engines. Application of TBCs has allowed increases in turbine efficiency by allowing increases in hot section temperatures. The TBC system is a multi-layered structure consisting of a super alloy substrate, a metallic bond coat, a thermally grown oxide layer (TGO) and the TBC itself. The TBC is typically yttria stabilized zirconia (YSZ) and is applied by either plasma spraying or by electron beam physical vapor deposition (EBPVD).

In die casting industry, the casting molds are usually coated to extend die life by increasing the wearing resistance so that the die maintenance, machine downtime and labor costs are reduced. The coating materials are usually chromium carbide, chromium 
nitride or titanium nitride. The commonly used coating process is Physical Vapor Deposition (PVD), Chemical Vapor Deposition (CVD) or Thermo-Reactive Deposition \& Diffusion (TRD \&D).

In the cutting tools industry, diamond coated cutting tools significantly improve productivity. Crystalline diamond coating can increase tool life 10 to 20 times in the process of non-ferrous material machining and more over uncoated tools with faster material removal, more reliable unattended high precision machining and reduced use of coolants in recommended materials.

Coatings have traditionally done an excellent job of doing what they were designed to do: prolong life by protecting component parts from oxidation and corrosion, erosion by particulate debris, and other potential hazards. The sacrifice of the virtue is the exposure of the coating to severe working conditions. The material properties of the thin film coatings would degrade after a period of time of working under the extreme environment. The neglect of the degradation will cause the failure of the coating, which will further cause the catastrophic failure of the whole component part.

For the gas turbine, hot section component materials and coatings degrade when they are exposed to service. The extent of degradation depends on both metal temperature and service time. In a cooled component, the material and coating degradation varies from location to location. The extent of in-service degradation increases with increasing metal temperature and/or service time. In-service material and coating degradation lowers mechanical properties, which lower the service life of components. 


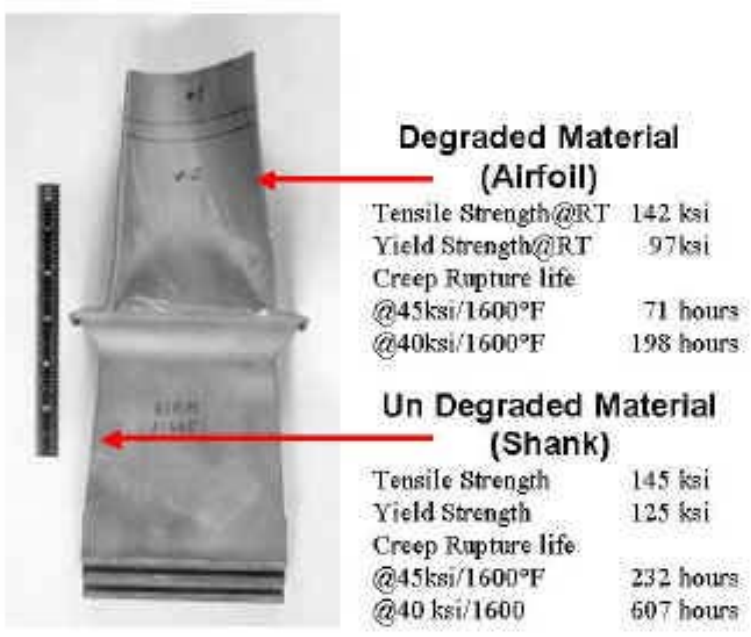

Figure 1.4 Degradation of material properties of a GTD 111 DS First Stage Blade after 49,000 hours of service.

(Excerpted from http://www.swri.edu/4org/d18/mechflu/planteng/gasturb/conditas.htm)

Various methods have been developed to assess the degradation of the thin film coating. Micro - indentation is one of the most powerful and popularly used. There are two main research areas in the thin film - substrate system: one is to evaluate the interfacial fracture toughness between the thin film coating and substrate material (Aditad Vasinaonta and Jack L. Beuth, 2000 [38]); another is to evaluate the intrinsic thin film material mechanical properties. As for the latter one, it is concluded that as long as the indentation depth is $10 \%$ to $20 \%$ of the thin film thickness, the effect of substrate is negligible. The behavior of the indentation parameters is essentially the responding of the thin film material.

Another incentive to apply indentation technique to thin film research is from the processing consideration. In recent years various sophisticated thin film deposition techniques have been developed in response to demands for better materials for high technological applications. These techniques, such as: sputtering, vapor deposition, ion 
implantation, laser glazing and other modern surface modification techniques, involve tailored control of the mechanical and structural properties of the material involved. With respect to mechanical properties, these kinds of control depend on a trial and error approach and little is known fundamentally about the relationships between the film stress, hardness, yield strength, elastic modulus and the film thickness. The mechanical properties of thin film material are different from those of the bulk material due to the different processing. How to find the thin film mechanical properties has been a delicate problem. The traditional uniaxial test is impossible to be applied in this field due to the size of the specimen. The micro indentation technique is emerging as one of the popular ways to study the elastic and plastic deformation of thin films on substrates.

During the last decade a new generation of indentation devices, so-called ultralow load or depth-sensing indentation systems [see for example Pethica et al. (1983) and Loubet et al. [6] (1984)], have been developed in order to make possible in situ testing of mechanical properties of thin film coatings. The development of an ultra low load indentation system (nano indentation) increased the use of hardness testing on small volumes of material, for example, electronics, corrosion protection coatings, paints and wear-resistant coatings for tooling and other tribological applications. One of the most important aspects of the instrumented indentation is the continuous reading of the applied load versus the indentation depth during the whole indentation cycle, which also enables extraction of information about the elastic properties of the material. The details are to be discussed in chapter 2 .

\subsection{Two theoretical branches of current indentation research}


Indentation methods are finding increasing use in the study of mechanical properties of bulk and thin-film materials over a wide range of size scales. There are basically two theoretical branches applied in indentation research, which are classified by the indentation size scale. One is traditional continuum mechanics while the other is newly developed strain gradient plasticity theory $[45,46,47,50]$.

Continuum mechanics has enjoyed a tremendous success in many disciplines of engineering, in which typical length scales of components and deformation are larger than millimeters. In recent years, continuum mechanics has been applied to microelectronic industry, where the characteristic length scales are very small, typically from 0.1 to 10 microns. Accordingly, several experiments have been developed to measure mesoscale mechanical properties.

Recent experiments, however, have shown that materials display strong size effects when the characteristic length scale associated with non-uniform plastic deformation is on the order of microns. For example, Fleck et al. (1994) $[45,46]$ observed in the twisting of thin copper wires that the scaled strength increases by a factor of three as the wire diameter decreases from 170 to 12 microns, while the increase of work hardening in simple tension is negligible. In bending, of ultra thin beams, Stolken and Evans (1998) [47] observed a significant increase in the normalized bending hardening as the beam thickness decreases from 100 to 12.5 microns, while data from simple tension displays no size dependence. For an aluminum-silicon matrix reinforced by silicon carbide particles, Lloyd (1994) [48] observed a substantial strength increase when the particle diameter was reduced from 16 to 7.5 microns with the particles volume fraction fixed at $15 \%$. More convincing experimental evidence of the size dependence of 
material behavior at the micron level comes from the micro-indentation or nanoindentation hardness test. The measured indentation decreases from 10 microns to 1 micron (Nix, 1989; Stelmeshenko et al., 1993 [49]; Ma and Clarke, 1995; Poole et al., 1996 [50]; McElhaney et al., 1998 [51]).

The classical plasticity theories cannot predict this size dependence of material behavior at the micron or nano scale because their constitutive models possess no internal length scale. The predictions based on the classical plasticity theories for non-uniform deformation (e.g. twisting, bending, deformation of composites, micro- or nanoindentation) do not exhibit size dependence after normalization. However, there is an impending need to deal with design and manufacturing issues at the micron level, such as in thin films where film thickness is on the order of 1 micron or less; sensors, actuators and micro-electromechanical systems (MEMS) where the entire system size is less than 10 microns; microelectronics packaging where features are smaller than 10 microns; advanced composites where particle or fiber size is on the order of 10 microns; as well as in micro-machining. The current design tools, such as finite element analysis (FEA) and computer aided design (CAD), are based on classical continuum theories, which may not be suitable at such a small length scale. On the other hand, it is still not possible to perform quantum and atomistic simulations on realistic time and length scales, which are required for the micron level structures. A continuum theory for micron level applications is thus necessary to bridge the gap between conventional continuum theories and atomistic simulations. Strain gradient plasticity represents such a theory that connects classical plasticity to dislocation mechanics.

The following chart gives a schematic scale idea of which theory to be applied. 


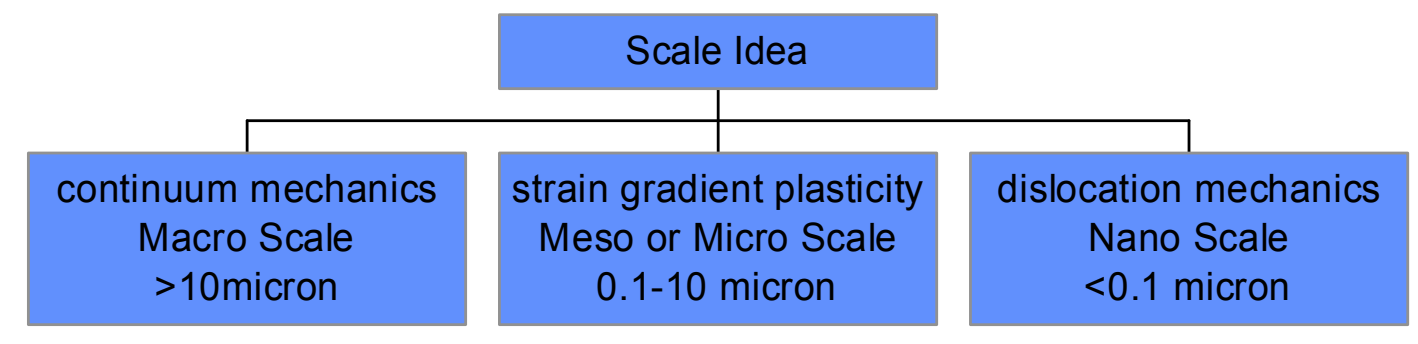

\subsection{Objectives}

In this research, based on a series of finite element (FE) indentation simulations of various materials, we aim to establish an indentation test methodology to obtain material surface mechanical properties, i.e. the stress-strain curve. The surface displacement fields from the FE simulations combined with indentation load-depth curve will be used to develop the proposed methodology to obtain bulk material stress-strain curve. The stress-strain curve is assumed to be a power-law hardening type with three definitive parameters: E (Young's modulus), $\sigma_{\mathrm{y}}$ (Yield Stress) and n (Strain hardening exponent).

Furthermore, the methodology will be extended to determine the mechanical properties (i.e. stress-strain curve) of thin film coating. By studying the loaddisplacement curve (P-hs curve), surface deformation besides the indenter, we are trying to extract the surface coating mechanical properties from indentation tests which will provide a simple, non destructive way to evaluate the thin film coating degradation. This proposed methodology would have enormous potential application in the gas turbine, diecasting, and cutting tools and other coating related industries.

We will use nonlinear, incremental FEM simulation of the micro indentation procedure to develop and verify the proposed methodology for stress-strain determination. The FEM is useful for this purpose because it provides a convenient and 
straightforward way to vary material properties over a wide range and the analysis also provides direct information on stress, strain, displacement, reaction force, contact opening, etc.

Since in polycrystalline materials contact areas due to nanoindentation may lie well inside a single grain or grain boundary, the properties obtained may be those of the indented grain or grain boundary only. While such measurements are desirable in many cases, an evaluation of bulk properties from nanoindentation is not straightforward. Macro indentation experiments, on the other hand, do represent the bulk behavior of the material, in which a large amount of grains are indented. Since the stress-strain curve is based on the macro scale assumptions, i.e. the material is isotropic, homogeneous; continuum mechanics is employed in the analysis. Therefore, relatively large indenter, who will cover at least several grains on the specimen surface, should be used to minimize the size effect of the tested materials and the macro response of the material is detected instead of local grain or grain boundary properties.

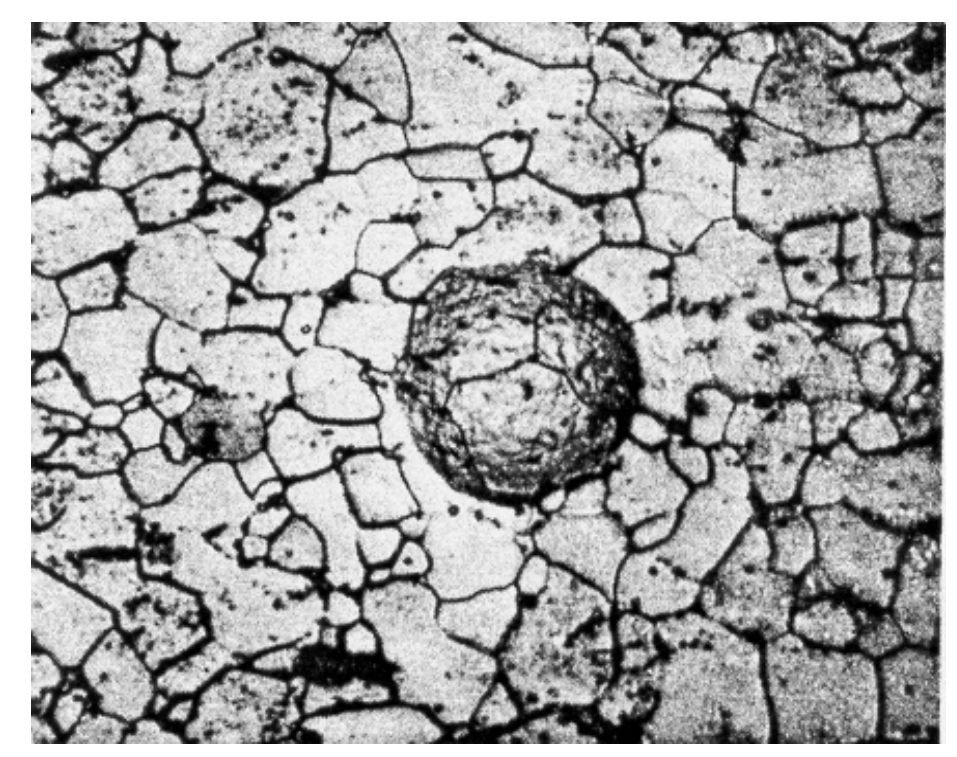


Figure 1.5 Spherical indent in 1015 steel $(20 \mu \mathrm{m}$ grain size $)$ obtained at a force of $2 \mathrm{~N}$ using a $254 \mu \mathrm{m}(0.010 \mathrm{in})$ diameter indenter. Notice that the progressive ball indentation at lowest depth increment should cover more than three grains in order to obtain macroscopic stress-strain properties. (Courtesy of [54]) 


\section{Chapter 2}

\section{Literature Review}

\subsection{Analytical solution development of indentation}

The nature of the stresses arising from the contact between two elastic bodies was first studied by Hertz in 1881 . His theory is found to be very accurately describing the stress, strain, and displacement fields in the elastic specimen by comparing with the finite element simulation results. Details are to be discussed in Chapter 4 and Hertz theory is to be served as verification of current finite element modeling. The problem of determining the distribution of stress within an elastic half space when it is deformed by the normal pressure against its boundary of a rigid punch have been considered first by Boussinesq in 1893. Another major contribution was made by Ian N. Sneddon [1] who derived general relationships among the load, displacement, and contact area for any punch that can be described as a solid of revolution of a smooth function. For flat-ended cylindrical punch problem, Sneddon showed the relationship between the applied load (P) and indentation depth (hs) as:

$$
\begin{aligned}
& P=\frac{2 E a h s}{1-v^{2}} \\
& \frac{d P}{d h_{s}}=\frac{2 E a}{1-v^{2}}
\end{aligned}
$$

In Equation (2.1), a is the actual contact radius at maximum load, which, for the case of a cylindrical punch, is equal to the radius of the indenter. E and $v$ are the Young's modulus and Poisson's ratio of the indented specimen respectively. This relationship is developed later to determine the elastic property of the specimen by loaddepth sensing indentation experiment. From their theories, the elastic stress fields 
generated by an indenter, although complex, are well defined within the terms of the classical theory of elasticity.

However, the nature of general materials' elastoplastic property challenges researchers more. K.L. Johnson [2,3] postulated that the analysis of Hill [4, chapter 5], for the expansion of a sphere cavity in an elastic-plastic material could be applied to the hemispherical radial mode of deformation observed in indentation tests by replacing the cavity with an incompressible, hemispherical core of material directly beneath the indenter of radius equal to the contact circle. Surrounding the core is a hemispherical plastic zone, which connects with the elastically strained material at some larger radius $\mathrm{c}$.

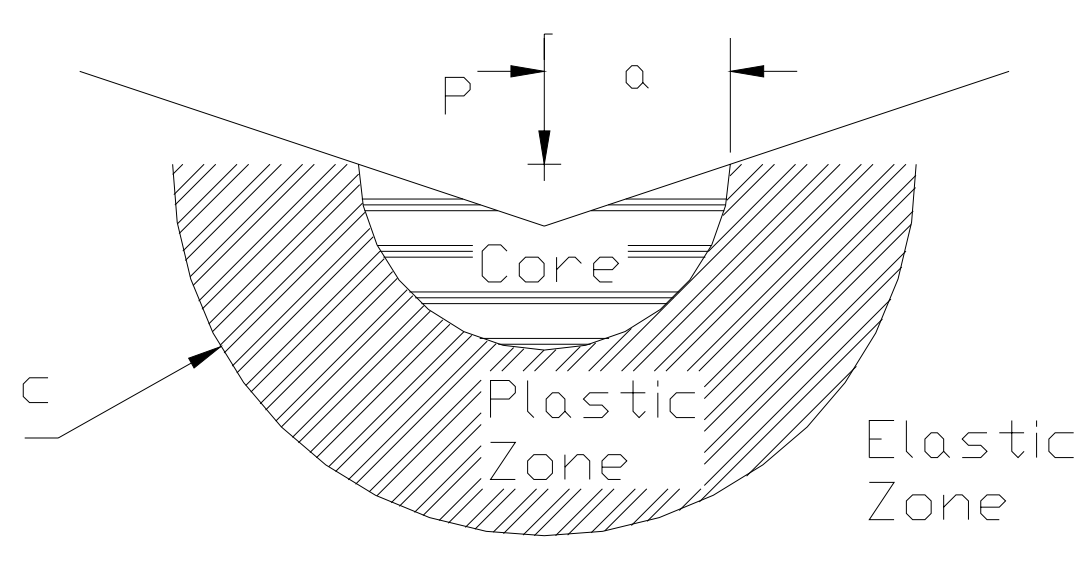

Figure 2.1 Johnson's elastic-plastic model [2,3].

The volume of the material displaced by the indenter is accommodated by radial displacement du(a) of material at the moving boundary of the rigid, hydrostatic core. These displacements give rise to stresses that are sufficiently high to cause plastic 
deformation in the surrounding material - the plastic zone. Within the plastic zone, $\mathrm{a}<\mathrm{r}<\mathrm{c}$, the stresses are given by Hill as:

$$
\begin{aligned}
& \frac{\sigma_{r}}{Y}=-2 \ln \left(\frac{c}{r}\right)-\frac{2}{3} \\
& \frac{\sigma_{\theta}}{Y}=-2 \ln \left(\frac{c}{r}\right)+\frac{1}{3}
\end{aligned}
$$

Radial displacement of material at the boundary of the core $\mathrm{r}=\mathrm{a}$, according to Hill, can be found from:

$$
\frac{d u(a)}{d c}=\frac{\sigma_{Y}}{E}\left[3(1-v) \frac{c^{2}}{a^{2}}-2(1-2 v) \frac{a}{c}\right]
$$

The elastic-plastic boundary is located by

$$
\frac{E}{\sigma_{Y}} \tan \beta=6(1-v)\left(\frac{c}{a}\right)^{3}-4(1-2 v)
$$

where $\beta$ is the angle between the tangential line and initial surface.

The conclusion above has been restricted to elastic - perfectly plastic solids which have a constant yield stress $\sigma_{\mathrm{Y}}$ in simple compression. It cannot be applied to material with strain hardening plastic behavior.

Modeling indentation contact that includes plasticity effect is a much more complex problem. Since the constitutive equations are nonlinear and a number of material parameters must be included to describe material behavior (e.g., yield strength and work hardening exponent), analytical solutions are not easily obtained. Previous theoretical treatments are limited because of the simplifying assumptions required to make such analysis tractable. As a result, much of our understanding of the importance of plasticity in indenter contact problems has been derived through experimentation and finite element simulation. 


\subsection{Determination of Young's modulus by load-depth sensing indentation}

For the past two decades, the advent of nano- and micro- scale science, engineering and technology coupled with substantial progress in instrumentation has resulted in 'instrumented' indentation or 'load-depth sensing' indentation. It primarily consists of a controlled load (P) applied through a diamond tip that is in contact with a specimen. The penetration depth $\left(\mathrm{h}_{\mathrm{s}}\right)$ of the tip into the material is recorded as a function of the applied load.

There is no question that the loading part is elastic-plastic response. The unloading part is usually considered pure elastic rebound of the material. It is only related to the elastic property of the material. If the area in contact is assumed to remain constant during initial unloading, the elastic behavior may be modeled as that of a blunt punch indenting an elastic solid. Loubet et al. [6], Doerner and Nix [8] adopted the solution of Sneddon [1] and equated the projected area of contact with the indenter to the area of the flat-ended punch. They obtained: 


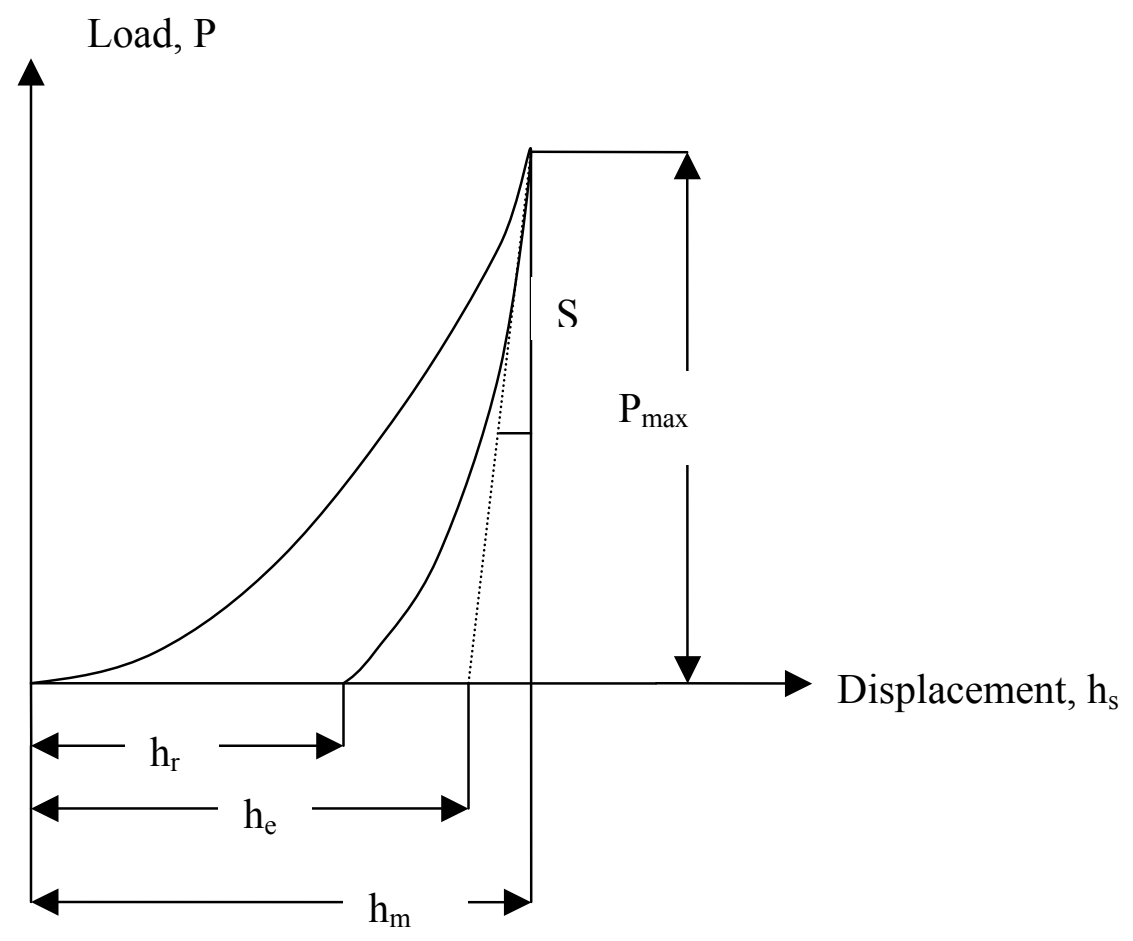

Figure 2.2 Schematic drawing of load displacement curve.

$S=\frac{d P}{d h_{s}}=2 E_{r} \sqrt{\frac{A}{\pi}}$

where

$\frac{1}{E_{r}}=\frac{1-v^{2}}{E}+\frac{1-v_{0}^{2}}{E_{0}}$

and $E_{r}$ is called reduced modulus or combined modulus, $S=d P / d h_{s}$ is the experimentally measured stiffness of the upper portion of the unloading data, which is the slope of the curve fitted straight line of the initial part of unloading, $\mathrm{A}$ is the projected contact area of the indenter at maximum loading condition, E and $v$ are Young's modulus and Poisson's ratio for the specimen, and $\mathrm{E}_{0}$ and $v_{0}$ are the same parameter for the indenter. 
A.C. Fischer-Cripps [7] showed that the substitution of reduced modulus in Equation (2.5) for indentation test data is valid. Because of the utilization of the slope or unloading stiffness, it makes no difference whether or not the deflection of the indenter is accommodated explicitly or transferred to that occurring within the specimen by artificially reducing the specimen modulus from its true value to lower value, the reduced modulus.

Usually the indenter is assumed to be perfectly rigid and $\mathrm{E}_{0}=\infty$. So, the Equation (2.6) becomes:

$E_{r}=\frac{E}{1-v^{2}}$

and plug into Equation (2.5), we get:

$$
E=\frac{\left(1-v^{2}\right)}{2} \sqrt{\frac{\pi}{A}} \frac{d P}{d h_{s}}
$$

Pharr, Oliver and Brotzen [9] have shown that Equation (2.8) is applicable to any indenter that can be described as a body of revolution of a smooth function.

To evaluate independently the projected contact area A, A.K. Bhattacharya and W.D. Nix [10] proposed a simple empirical method based on extrapolating the initial linear portion of the unloading curve to zero load and using the extrapolated depth with the indenter shape function to determine the contact area.

One of the more commonly used methods to get contact area by analyzing micro indentation load-displacement data is that of Oliver and Pharr [12], which expands on ideas developed by Loubet et al. [6] and A. K. Bhattacharya and Nix [10]. They found the load-displacement curves during unloading are not linear for most materials, even in 
the initial stages. The analysis begins by fitting the unloading curve to the power-law relation $P=B\left(h_{s}-h_{f}\right)^{m}$

where $\mathrm{P}$ is the indentation load, $\mathrm{h}_{\mathrm{s}}$ is the displacement, $\mathrm{B}$ and $\mathrm{m}$ are empirically determined fitting parameters, and $\mathrm{h}_{\mathrm{f}}$ is the final displacement after complete unloading. By differentiating above equation at the maximum depth of penetration, $\mathrm{h}_{\mathrm{s}}=\mathrm{h}_{\mathrm{m}}$, giving stiffness S:

$S=\frac{d P}{d h_{s}}\left(h_{s}=h_{m}\right)=m B\left(h_{m}-h_{f}\right)^{m-1}$

The depth along which contact is made between the indenter and the specimen, $\mathrm{h}_{\mathrm{c}}$, can also be estimated from the load-displacement data using:

$h_{c}=h_{m}-\varepsilon \frac{P_{m}}{S}$

where $\mathrm{P}_{\mathrm{m}}$ is the peak indentation load and $\varepsilon$ is a constant which depends on the geometry of the indenter. With these basic measurements, the projected contact area, A, is derived by evaluating an indenter shape function at the contact depth, $\mathrm{h}_{\mathrm{c}}$, that is $\mathrm{A}=\mathrm{f}\left(\mathrm{h}_{\mathrm{c}}\right)$. Finally, substitute $\mathrm{S}$ in Equation (2.10) and the projected area A into Equation (2.8) to obtain the Young's modulus of the specimen.

It is important to note these equations were derived from pure elastic contact solution derived by Sneddon, and how well they work for elastic/plastic indentation is not entirely clear. One important way in which the elastic solution fails to properly describe elastic/plastic behavior concerns pileup and sink-in of material around the indenter. In the pure elastic contact solution, material always sinks in, while for elastic/plastic contact, material may either sink in or pile up. Since this has important effects on the 
indentation contact data, it is not surprising that the Oliver-Pharr method has been found to work well for hard ceramics, in which sink-in predominates, but significant errors can be encountered when the method is applied to soft metals that exhibit extensive pileup.

A.Bolshakov and G.M. Pharr [16] discussed the influences of pileup on the measurement of Young's modulus and pointed out that when pileup is large, the areas deduced from analysis of the load displacement curves underestimates the true contact areas by as much as $60 \%$. This, in turn, leads to overestimate the hardness and elastic modulus. The parameter $h_{f} / h_{\max }$, which can be measured experimentally and correlated with the material parameters $\mathrm{E}, v, \sigma_{\mathrm{y}}$ and $\eta(\eta=d \sigma / d \varepsilon)$ which control indentation deformation, can be used as an indication of whether or not pileup is an important factor. Pileup is significant only when $h_{f} / h_{\max }>0.7$ and the material does not appreciably work harden. When $h_{f} / h_{\max }<0.7$, or in all materials that moderately work harden, pileup is not a significant factor and the Oliver-Pharr data analysis procedure can be expected to give reasonable results. They used Finite Element Method to complete their analysis and the real contact area is available by examining of the contact profiles in the finite element mesh.

Recently, Hay et al. [32,33] have shown that since the boundary conditions used to derive elastic contact models employed in indentation allow for inward displacement of the surface, a correction factor, $\beta$, needs to be added to Equation (2.5) which becomes:

$$
S=\frac{d P}{d h_{s}}=2 \beta E_{r} \sqrt{\frac{A}{\pi}}
$$

So the formula to find Young's modulus (2.8) becomes: 


$$
E=\frac{\left(1-v^{2}\right)}{2 \beta} \sqrt{\frac{\pi}{A}} \frac{d P}{d h_{s}}
$$

For a conical indenter with half apex angle $(\gamma)$ less than $60^{\circ}$, the function $\beta$ reads [32]:

$$
\beta=1+\frac{2(1-2 v)}{4(1-v) \tan \gamma}
$$

while for larger cone angles, i.e., a Berkovich indenter, the function $\beta$ reads [32]:

$$
\beta=\pi \frac{\pi / 4+0.1548 \cot \gamma \frac{1-2 v}{4(1-v)}}{\left[\pi / 2-0.8311 \cot \gamma \frac{1-2 v}{4(1-v)}\right]^{2}}
$$

For a spherical indenter the function $\beta$ is given by [33]:

$$
\beta=1+\frac{2(1-2 v) a}{3 \pi(1-v) R}
$$

where $\mathrm{a}$ is the contact radius and $\mathrm{R}$ is the radius of the sphere indenter.

\subsection{Correlation between yield strength and hardness}

The development of indentation methodologies for the micro mechanical characterization of materials requires a precise understanding of the correlation between uniaxial mechanical properties and hardness. One of such fundamental correlations was found by Tabor [39] for pyramidal (Vickers) indenters. Considering indentation experiments conducted in specimens of pure copper and mild steel which were previously subjected to different amounts of strain hardening, Tabor proposed that hardness is, to a great extent, proportional to the uniaxial stress at a plastic strain of 0.08 . Namely,

$$
H=C \sigma_{r}
$$

where $\mathrm{H}$ is the Vickers hardness of the material (as evaluated by the ratio between maximum applied load and projected contact area, which is different from the definition 
introduced in section 1.1), $\mathrm{C}=3.3$, and $\sigma_{r}$ is the uniaxial stress corresponding to a characteristic uniaxial strain $\left(\varepsilon_{r}\right)$ of 0.08 . Over the years, Equation (2.17) was simplified leading to conception that hardness is proportional to the yield strength. Obviously this empirical simplification is inaccurate for materials exhibiting considerable strain hardening, as in the case of the metals that were originally studied by Tabor.

\subsection{Methodology to extract stress-strain curve from indentation}

In general, the material mechanical properties, e.g. Young's modulus and yield strength can be evaluated from its uniaxial test stress strain curve ( $\sigma-\varepsilon$ curve). What is the relationship between the $\sigma-\varepsilon$ curve and indentation test data?

A typical work done by M. Dao and Suresh in MIT [13] is to use FEM to study the cone indentation's load displacement curve responses of 76 different combinations of elasto-plastic properties that encompass the wide range of parameters commonly found in pure and alloyed engineering metals. They picked up three variables from the P-hs curve which are:

$$
\begin{aligned}
& C=\frac{P}{h s^{2}} \\
& S=\left.\frac{d P}{d h s}\right|_{h_{m}}
\end{aligned}
$$

$\frac{h_{r}}{h_{m}}$

where $C$ is the loading curvature, $S$ is the stiffness of the initial unloading and $h_{r} / h_{m}$ is the ratio of residual depth to maximum depth. They assume the three parameters are related to the material elasto-plastic property $\left(\mathrm{E}, v, \sigma_{\mathrm{y}}\right.$ and $\left.\mathrm{n}\right)$. From the FEM simulation results, analytical expressions were derived to relate indentation data to elasto-plastic properties of the material through dimensional analysis. Forward and reverse analysis algorithms 
were thus established. However, these plastic properties are very strongly influenced by even small variations in the parameters extracted from instrumented indentation experiments.

Yang-Tse Cheng and Che-Min Cheng [17] pointed out that even for different material properties, it is possible to get very resembling load-displacement response in the indentation test. Consequently the feasibility of extracting $\sigma-\varepsilon$ curve from loaddisplacement curve alone is questionable.

In the paper by A.E. Giannakopoulos and S. Suresh [19], they identified a general theoretical framework for instrumented sharp indentation and outlined a methodology, which enables the determination of elastic and plastic properties of materials by employing instrumented sharp indentation. A key feature of the method is that it provided unique correlations between the penetration depth hs and true contact area A for commercially available sharp indenters. It circumvented the need for contact area measurement through visual observations while, at the same time, taking into account pile-up and sink-in.

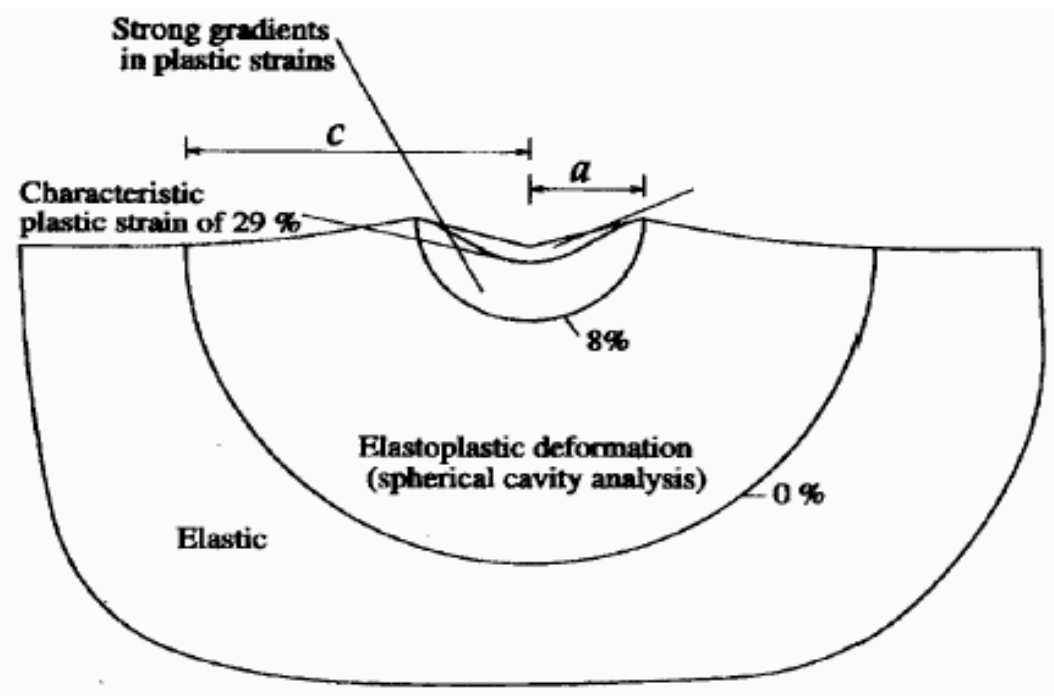

Figure 2.3 Different regimes of plastic flow at the tip of a sharp indenter [19]. 
Another important concept is that there exists a unique characteristic equivalent plastic strain, which separates different modes of plastic deformation directly beneath the sharp indenter. This is schematically shown in Figure 2.3, which is similar to Johnson's cavity model. The innermost region comprises a zone where the material is 'cut' by the sharp indenter; this cutting mode is amenable to analysis by means of the classical slipline theories of rigid-perfectly plasticity. Surrounding the region is a zone of strong gradients in plastic strains which is engulfed by an elastoplastic regime where the socalled 'cavity' model of Johnson can be applied to extract the hemi-spherically shaped elastoplastic boundary. A unique characteristic strain separates the innermost 'cutting' region from its surroundings. In Giannakopoulos's approach, this characteristic plastic strain is set to be 0.29 . Invoking the existence of this characteristic strain is a key step in the determination of mechanical properties by sharp indentation in their approach.

With the theoretical framework, a step-by-step method is presented for the estimation of bulk mechanical properties. Properties such as Young's modulus, compressive yield strength, strain hardening exponent, strength at a plastic strain of 0.29 and hardness can be determined in-situ from an automated recording of P-hs curves.

Another research group led by Fahmy M. Haggag $[14,15]$ in Oak Ridge National Laboratory has developed an automated ball indentation (ABI) method for stress-strain curve determination. Their Stress-Strain Microprobe (SSM) system is capable of determining the tensile deformation and fracture properties based on the ABI method. It involves strain-controlled multiple indentations at the same location on the material surface by a small spherical indenter. A series of equations were derived to relate the indentation data with the stress-strain curve parameters. 
At first the yield stress was calculated by the simple equation:

$\sigma_{Y}=\beta_{m} A$

where $\beta_{m}$ is a constant for a given class of materials. The value of $\beta_{m}$ for each class of materials is determined independently using yield strength obtained from standard tensile tests, and value of A obtained from ABI test. A is obtained by linear regression analysis following the relationship:

$P / d_{t}^{2}=A\left(d_{t} / D\right)^{m-2}$

where

$\mathrm{P}$ is the applied indentation load;

$\mathrm{m}$ is the Meyer's coefficient and A is a material parameter obtained from the regression;

$d_{t}=2 \sqrt{D h_{s}-h_{s}{ }^{2}}$

$\mathrm{D}$ is the diameter of the ball indenter

The true stress $\left(\sigma_{t}\right)$ and plastic strain $\left(\varepsilon_{\mathrm{p}}\right)$ values are calculated using the following equations:

$$
\begin{gathered}
\varepsilon_{p}=0.2 \frac{d_{p}}{D} \\
\sigma_{t}=\frac{4 P}{\pi d_{p}{ }^{2} \delta}
\end{gathered}
$$

where $\mathrm{d}_{\mathrm{p}}$ and $\delta$ are parameters related to indentation data. They are computed by iterating the following equations: 


$$
\begin{aligned}
& d_{p}=\sqrt[3]{(2.735 P D) \frac{\left(\frac{1}{E}+\frac{1}{E_{0}}\right)\left(4 h_{p}{ }^{2}+d_{p}{ }^{2}\right)}{\left(4 h_{p}{ }^{2}+d_{p}{ }^{2}-4 h_{p} D\right)}} \\
& \delta=\left\{\begin{array}{cc}
1.12 & \phi \leq 1 \\
1.12+\tau \ln \phi & 1<\phi \leq 27 \\
2.87 \alpha_{m} & \phi>27
\end{array}\right.
\end{aligned}
$$

where:

$$
\begin{aligned}
\tau & =\frac{2.87 \alpha_{m}-1.12}{\ln (27)} \\
\phi & =\varepsilon_{p} E_{\text {spec }} / 0.43 \sigma_{t}
\end{aligned}
$$

$\alpha_{\mathrm{m}}$ is material dependent and varies between 0.9 and 1.25 for structural steels.

Although the ABI technique has been applied as an in-situ testing instrument for non-destructive assessment of local stress-strain behavior of operating structural components, the methodology is empirical and lack of theoretical support. The concept (i.e. Equation (2.22)) was based on Tabor's experimental work in 1950's [39]. Most of the coefficients are based on empirical, curve fitting or regression data for a given class of materials. The effect of surface deformation (piling up or sinking in) is not considered. Also, since the size of the indenter is large (diameter varying from $0.254 \mathrm{~mm}$ to $1.575 \mathrm{~mm}$ ), it is hard to apply to determine mechanical properties of thin film materials.

\subsection{Surface deformation analysis}

As mentioned before, it is questionable to extract the stress-strain relation from load-displacement (P-hs) curve obtained from indentation test. More experimentally obtainable indentation features are needed to fulfill the objective. 
In recent years, some researchers have put their focus on the surface deformation mode during the indentation test. They have found the pile-up or sink-in mode during indentation most depend on the strain-hardening exponent of the material. The research by J. Alcala [18] pointed out that sinking-in predominates in materials where $n>0.2$. Such surface deformation modes influence hardness and elastic modules measurements as the true contact area between the indenter and the specimen increases in the case where piling-up predominates and deceases in the event that sinking-in occurs. Moreover, the evaluation by using the instrumented indentation requires a precise knowledge of the surface deformation modes, as errors of up to $30 \%$ can be introduced in the computation of contact area if the development of piling-up and sinking-in is not taken into account.
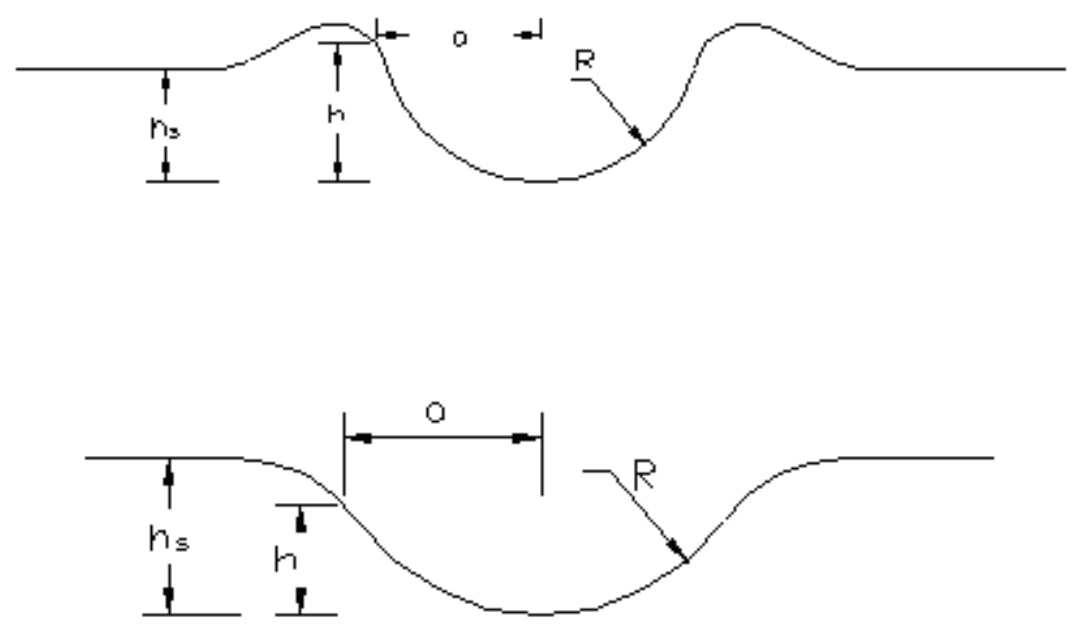

Figure 2.4 Schematic drawing of piling-up and sinking in of material around spherical indents.

A quantification of piling-up and sinking-in around spherical indenter is given by the parameter $\mathrm{c}$ in the equation:

$h=h_{s} c^{2}$ 
where $h_{s}$ is the maximum depth of penetration underneath the original surface and $h$ sets the location of the contact area. It is obvious that the parameter $\mathrm{c}^{2}-1$ provides the amount of piling-up $\left(\mathrm{c}^{2}-1>0\right)$ or sinking-in $\left(\mathrm{c}^{2}-1<1\right)$ as a fraction of the maximum penetration of the indenter, $\mathrm{h}_{\mathrm{s}}$. Factor $\mathrm{c}^{2}$ is anticipated to depend on the strain hardening exponent, $\mathrm{n}, \mathrm{by}$ means of a continuous function. A master curve shown in Figure 2.5 was established to show the relation between $\mathrm{c}^{2}-1$ and $\mathrm{n}$ value, from which, the $\mathrm{n}$ value could be determined by examining surface deformation mode.

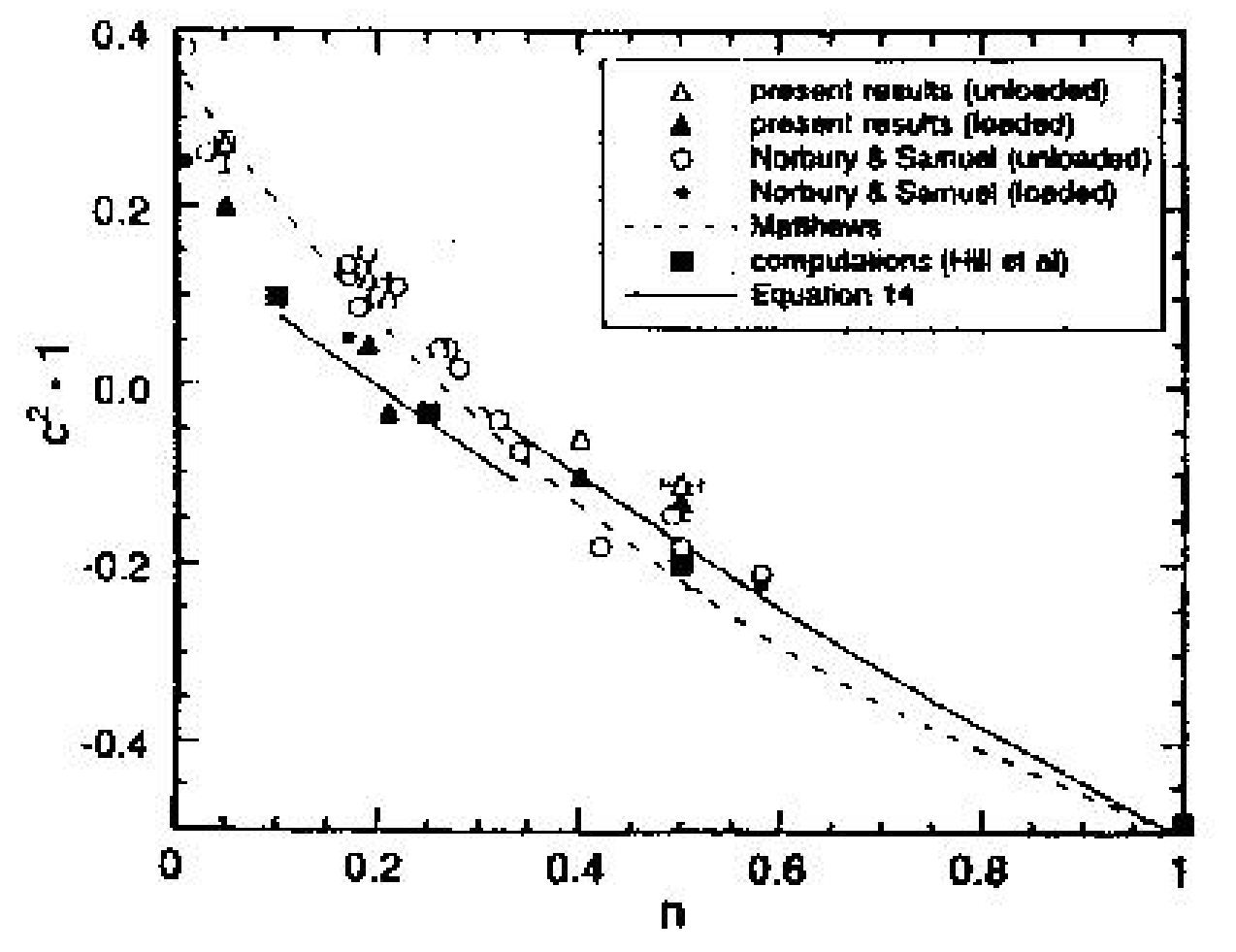

Figure 2.5 Dependence of the surface deformation parameter $\mathrm{c}^{2}-1$ for spherical indentation on the strain hardening exponent $\mathrm{n}[18]$.

Also an equation was given [18]:

$c^{2}=\left[\frac{2 / n-1}{4 / n+1}\right]\left[\frac{4 s+1}{2 s}\right]$ 
where $\mathrm{s}$ is a dimensionless parameter which depends on the pressure distribution over the contact surface.

There are scarce literatures to discuss the surface displacement field, either experimentally or theoretically. Only John H. Underwood described his method to measure residual stress using surface displacement around spherical indentation in reference [44] in 1973. The method uses optical interference to measure the permanent surface deformation around a shallow spherical indentation in a polished area on the metal specimen.

\subsection{Thin film - substrate system}

In recent years, various methods were developed to measure the thin film mechanical properties. For example, Kurt E. Petersen and C.R. Guarnieri [24] used optical experimental apparatus to measure mechanical resonant frequencies of the cantilever beam made of the thin film to be determined. Then they obtained the thin film Young's modulus by the relationship between the resonant frequency and the modulus on the cantilever beam. The most common case is that the thin film is usually bonded with the substrate. Moreover, its mechanical properties will degrade after a period of working in critical conditions (high temperature, high pressure, chemical reaction, etc). How to find the thin film mechanical properties while keeping the structural integrity? Micro- or nano- indentation has been an essential tool to solve this problem.

Recently, many experiment and analytical efforts have been made to measure the hardness and stiffness of a thin film in the presence of a substrate. In general, indentations with contact depths of less than $10 \%$ to $20 \%$ of the film thickness are needed in order to obtain intrinsic film properties and to avoid the so-called substrate effect [23]. 
This is often not practical as the thickness of films used in many cases continues to decrease. For example, the thickness of barrier films currently used in semiconductor devices is well below $50 \mathrm{~nm}$. Due to experimental equipment limitations, it is very difficult to perform indentation tests at such a shallow depth. It is inevitable for the substrate to affect the final measurements.

In the case of thin films on substrates, the linear relationship of initial unloading P-hs curve no long holds due to the varying contribution of the film and substrate to the measured compliance. The variation of overall compliance (1/S, reciprocal of stiffness) of a thin film/substrate composite was studied by Doerner and Nix [29]. Later King [5] used basis functions and an integral equation technique to perform elastic analysis for punches of different shapes and derived more accurate equations for the compliance in terms of the projected area of contact under the indenter. King's equation, when simplified for a perfectly rigid indenter, becomes:

$$
\frac{d h}{d P}=\frac{1}{\beta \sqrt{A}}\left\{\frac{1-v_{f}^{2}}{E_{f}}\left[1-\exp \left(-\frac{\alpha t_{f}}{\sqrt{A}}\right)\right]+\frac{1-v_{s}^{2}}{E_{s}}\left[\exp \left(-\frac{\alpha t_{f}}{\sqrt{A}}\right)\right]\right\}
$$

where $\mathrm{A}$ is the projected contact area, $\beta$ is a numerical factor related to the shape of the indenter and $\alpha$ a parameter dependent on the depth and available from a set of curves given by King [5].

A.K. Bhattacharya and W.D. Nix [11] pointed out that Equation (2.27) is in reasonable agreement with the FEM results for large depth of indentation. But for smaller depth of indentation, it appears that the agreement between Equation (2.27) and FEM results is bad. An approximate analysis by H. Gao et al. [30] yields the following expression for the reduced modulus of a film on substrate: 


$$
E_{r}=\frac{2 \mu_{e f f}}{1-v_{\text {eff }}}
$$

where

$$
\left\{\begin{array}{c}
v_{e f f}=v_{s}+\left(v_{f}-v_{s}\right) I_{1}\left(t_{f} / a\right) \\
\mu_{e f f}=\mu_{s}+\left(\mu_{f}-\mu_{s}\right) I_{0}\left(t_{f} / a\right)
\end{array}\right.
$$

Here, $\mu_{\mathrm{f}}$ and $\mu_{\mathrm{s}}$ are the shear modulus of the film and substrate respectively. The two functions, $\mathrm{I}_{0}$ and $\mathrm{I}_{1}$ are weight functions that reflect the substrate effect and take the following form:

$$
\begin{aligned}
& I_{1}(\xi)=\frac{2}{\pi} \arctan \xi+\frac{\xi}{\pi} \ln \frac{1+\xi^{2}}{\xi^{2}} \\
& I_{0}(\xi)=\frac{2}{\pi} \arctan \xi+\frac{1}{2 \pi(1-v)}\left[(1-2 v) \xi \ln \frac{1+\xi^{2}}{\xi^{2}}-\frac{\xi}{1-\xi^{2}}\right]
\end{aligned}
$$

where $\xi=\mathrm{t}_{\mathrm{f}} / \mathrm{a}$. Both of these functions approach unity when $\xi$ is very large (thick film and shallow indentation) and measured indentation modulus is equal to that of the film.

Xi Chen and Joost J. Vlassak [22] applied finite element method (FEM) to investigate the effect of the substrate on hardness and stiffness measurements for a variety of thin film systems. The comparison of his results with Gao's theoretical result shows that the weight function $\mathrm{I}_{0}$ in Equation (2.31) overestimates the substrate effect when the film is stiffer than the substrate and that the substrate influence is underestimated if the film is more compliant. 


\section{Chapter 3}

\section{Finite Element Modeling}

\subsection{Introduction to ABAQUS software package}

Commercial finite element analysis (FEA) software package ABAQUS ${ }^{\mathrm{TM}}$ version

6.3 is used in the indentation simulation. ABAQUS ${ }^{\mathrm{TM}}$ is developed by Hibbitt, Karlsson \& Sorensen, Inc. It is a suite of powerful engineering simulation programs, based on the finite element method, which can solve problems ranging from relatively simple linear analysis to the most challenging nonlinear simulations.

ABAQUS consists of two main analysis modules -ABAQUS/Standard and ABAQUS/Explicit. ABAQUS/Standard is a general-purpose analysis module that can solve a wide range of linear and nonlinear problems efficiently, accurately and reliably. ABAQUS/Explicit is a special-purpose analysis module that uses an explicit dynamic finite element formulation. It is suitable for short, transient dynamic events. The indentation procedure is assumed to be quasi-static problem, in which no rate effect is considered. So ABAQUS/Standard is employed in this work.

A complete ABAQUS analysis usually consists of three distinct stages: preprocessing, simulation and post processing.

ABAQUS/CAE is the complete ABAQUS environment that includes capabilities for creating ABAQUS models, interactively submitting and monitoring ABAQUS jobs and evaluating results. In our job, the ABAQUS/CAE is used as the preprocessor (Part, Property, Assembly, Step, Interaction, Load, Mesh, Job modules) and postprocessor (Visualization module).

\subsection{Nonlinear problem analysis in ABAQUS}


In the indentation simulation, there are two sources of nonlinearity: material nonlinearity and geometric nonlinearity.

Most metals have a fairly linear stress/strain relationship at low strain values but at higher strains the material yields, at which point the response becomes nonlinear and irreversible (material nonlinearity). Also, the indentation procedure produces large deformation in the solids underneath and near the indenter. The magnitude of displacement affects the response of the structure (geometry nonlinearity).

ABAQUS uses the Newton-Raphson method to obtain solutions for nonlinear problems. The solution is found by applying the specified loads gradually and incrementally working towards the final solution. Therefore, ABAQUS breaks the simulation into a number of load increments and finds the approximate equilibrium configuration at the end of each load increment. It often takes ABAQUS several iterations to determine an acceptable solution to a given load increment. The sum of all of the incremental responses is the approximate solution for the nonlinear analysis.

The load history for a simulation consists of one or more steps. Users define the steps, which generally consists of an analysis procedure option, loading options and output requests options. An increment is a part of a step. In nonlinear analysis the total load applied in a step is broken into smaller increments so that the nonlinear solution path can be followed. Users suggest the size of the first increment and ABAQUS automatically chooses the size of the subsequent increments. At the end of each increment the structure is in approximate equilibrium and results are available for writing to the restart, data or results files. In each increment, ABAQUS uses several iterations to attempt to find equilibrium solution. In a nonlinear analysis a step takes place over a 
finite period of "time", although this "time" has no physical meaning unless inertial effects or rate dependent behavior are important.

\subsection{Material mechanical behavior}

\subsubsection{Elasticity and plasticity}

In uniaxial tensile test, many metals have approximately linear elastic behavior at low strain magnitudes and stiffness of the material, known as the Young's or elastic modulus, is constant. At higher stress (and strain) magnitudes, metals begin to have nonlinear, inelastic behavior, which is referred to as plasticity. The plastic behavior of a material is described by its yield point and its post-yield hardening. In most metals the yield stress is 0.05 to $0.1 \%$ of the material's elastic modulus. The stiffness of a metal typically decreases dramatically once the material yields. A ductile metal that has yields will recover its initial elastic stiffness when the applied load is removed. Often the plastic deformation of the material increases its yield stress for subsequent loading: this behavior is called work hardening.

\subsubsection{Engineering and true stress-strain relationship}




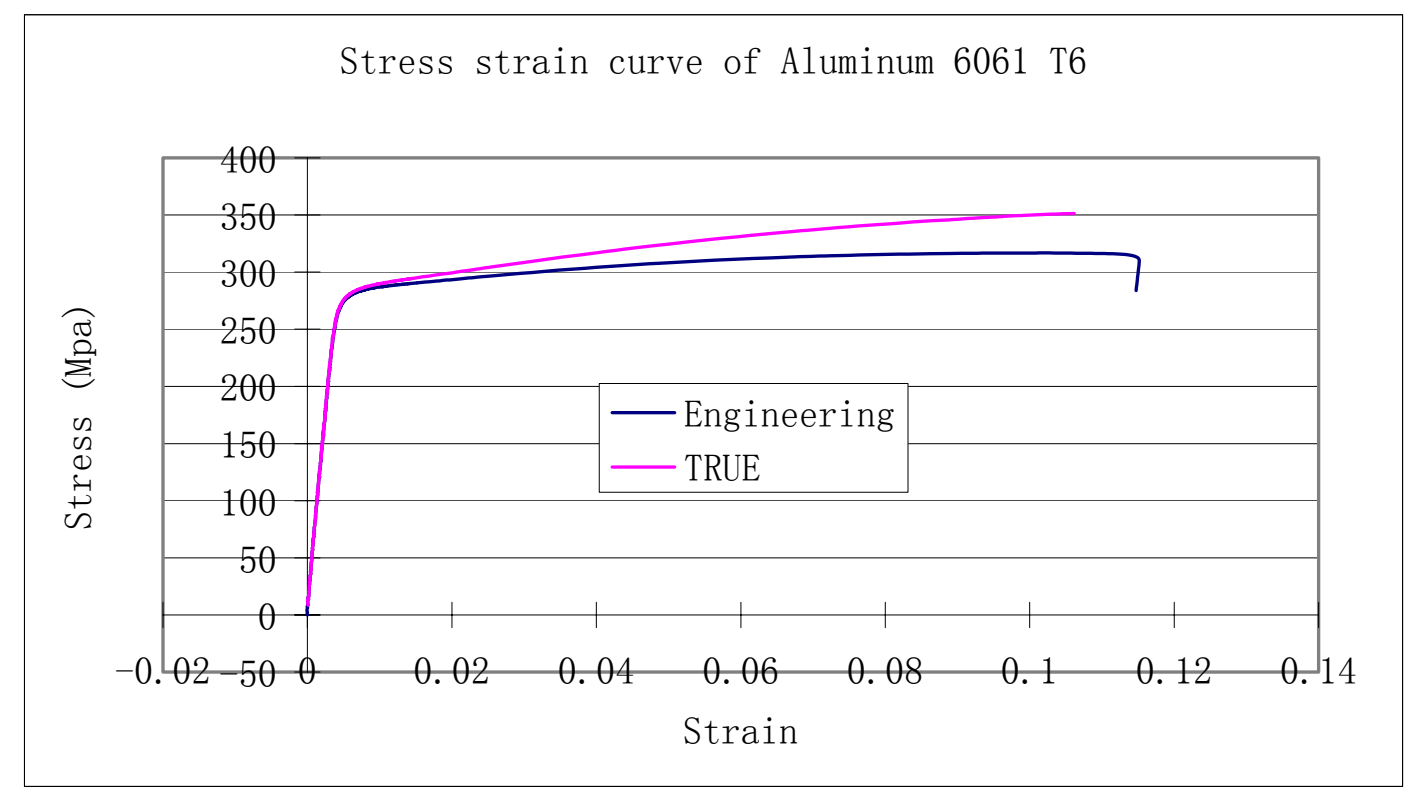

Figure 3.1 Engineering and true stress strain curve (Aluminum $6061 \mathrm{~T} 6$ ).

The Figure 3.1 is the uniaxial tension test data of Aluminum 6061-T6. The blue line represents engineering stress (s) and engineering strain (e), which are defined as:

$$
\begin{aligned}
& e=\frac{L-L_{0}}{L_{0}} \\
& s=\frac{P}{A_{0}}
\end{aligned}
$$

where $P$ is the load $(N), A_{0}$ is the original cross-section area $\left(\mathrm{mm}^{2}\right), L_{0}$ is the original length of the specimen, $\mathrm{L}$ is the length under loading condition.

The red line represents true stress $(\sigma)$ and true strain $(\varepsilon)$. The relationships between the engineering and true stress strain are:

$$
\begin{aligned}
& \varepsilon=\ln (1+e) \\
& \sigma=s(1+e)
\end{aligned}
$$

In finite element method, since the plastic deformation produces geometry nonlinearity and material nonlinearity, the true stress-strain curve is required as the 
material property input. Then ABAQUS applies these data into the constitutive equations to get the solution for stress, strain, and displacement in 3-dimentional state of stress.

\subsubsection{Mathematical models to describe stress-strain curve}

Different materials demonstrate different shape of stress strain curves, which then are described by different math models. Several commonly used math models to describe of the true stress-strain curve are:

a) Piecewise linear elastic - power-law plastic model

$$
\begin{aligned}
& \varepsilon=\left\{\begin{array}{cc}
\sigma / E & \sigma<\sigma_{y} \\
\frac{\sigma_{y}}{E}\left(\frac{\sigma}{\sigma_{y}}\right)^{1 / n} & \text { otherwise }
\end{array}\right. \\
& \text { or } \\
& \sigma=\left\{\begin{array}{cc}
E \varepsilon & \sigma<\sigma_{y} \\
\sigma_{y}\left(\frac{E}{\sigma_{y}}\right)^{n} \varepsilon^{n} & \text { otherwise }
\end{array}\right.
\end{aligned}
$$

where $\varepsilon$ is the total strain $\left(\varepsilon=\varepsilon_{\mathrm{e}}+\varepsilon_{\mathrm{p}}\right), \sigma_{\mathrm{y}}$ is yield strength and $\mathrm{n}$ is the power law strainhardening coefficient. This equation accurately describes the elastic regime of metallic materials and their subsequent plastic stress - strain curve of metals at least to intermediate values of plastic deformation. Also this equation is formulated so that the elastic behavior matches the plastic response at $\sigma=\sigma_{y}$. The existence of such welldefined yield strength allows one to model the response of metallic materials when subjected to different amounts of strain hardening.

The value of $\mathrm{n}$ represents the stage of the materials strain hardening and it varies from 0 to 1 for different materials. Two extreme cases are: $n=0$ represents elastic perfectly plastic material and $n=1$ represents pure elastic material. For average elastic plastic materials, $\mathrm{n}$ value lies between 0 and 1 , that is $0<\mathrm{n}<1$. For most engineering metallic materials, $0<\mathrm{n}<0.5$. 
b) Linear elastic - linear strain hardening plastic model

$\varepsilon=\left\{\begin{array}{cl}\sigma / E & \sigma<\sigma_{y} \\ \left(\sigma-\sigma_{y}\right) / m+\sigma_{y} / E & \text { otherwise }\end{array}\right.$

where $\mathrm{m}$ is the linear strain hardening coefficient.

However, there are seldom materials whose plasticity behavior in stress -strain curve is linear. So the application of the model is limited.

c) Another equation being used to describe the stress strain relation is:

$\sigma=\left\{\begin{array}{cc}E \varepsilon & \sigma<\sigma_{y} \\ \sigma_{y}+K\left(\varepsilon-\frac{\sigma}{E}\right)^{n} & \text { otherwise }\end{array}\right.$

d) Ramberg-Osgood model

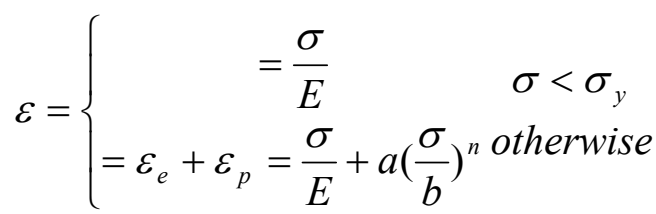

Both (c) and (d) equations divide the strain as the summation of the elastic strain and plastic strain.

In our work, the Equation (3.3) is used. So, once the $\mathrm{E}, \sigma_{\mathrm{y}}$ and $\mathrm{n}$ three parameters are determined, the whole stress strain curve is defined. Once the true stress-true strain curve is obtained, the ultimate tensile stress can be determined in an indirectly way. Since $n$ is equal to the true strain at the ultimate tensile stress of the material under tensile loading, which is $\varepsilon_{u}=n=\ln \left(1+e_{u}\right)$, thus the true ultimate tensile stress can be obtained from Equation (3.3):

$\sigma_{T S}=\sigma_{y}\left(\frac{E}{\sigma_{y}}\right)^{n} n^{n}$ 
The ultimate tensile strength (engineering stress), $\mathrm{S}_{\mathrm{UTS}}$, is then obtained from the equation:

$$
S_{U T S}=\frac{\sigma_{T S}}{1+e_{u}}=\sigma_{y}\left(\frac{E}{\sigma_{y}}\right)^{n} \frac{n^{n}}{1+e_{u}}=\sigma_{y}\left(\frac{E}{\sigma_{y}}\right)^{n}\left(\frac{n}{e}\right)^{n}
$$

In all the former research, it is acknowledged in consensus that the Poisson's ratio effect is negligible in the indentation analysis. The finite element simulation results from A. Bolshakov and G. M. Pharr [16] showed that varying Poisson's ratio in the range $0 \leq v \leq 0.45$ has only minor influence on the contact profile. The subsequent work focuses on how to relate the indentation data with the three parameters, E, $\sigma_{y}$ and $n$.

\subsection{Finite element modeling of indentation}

\subsubsection{Theoretical background}

Finite element simulations are performed for the problem of a rigid spherical indenter brought into contact against an infinite half space. Both the indenter and half space are modeled as axisymmetric geometry. The constitutive model of the half space is taken to follow $\mathrm{J}_{2}$-associated flow rule with rate-independent deformation and isotropic hardening. While the isotropic hardening has been regarded as an accurate model in prior finite element modeling of indentation, this assumption is merely a simplification of the true mechanical response of the material as the shape of the yield locus in most metals varies during plastic flow. M. Mata et al. [20] stated that 'the hypothesis of isotropic hardening is considered to provide accurate results in the analysis of loading histories where no reversed plastic deformation occurs well beyond the yield surface.'

The response is taken to be rate independent, so in the quasi-static calculations presented here no rate effects are represented. The influence of large deformation is 
included in the analysis by using geometry nonlinearity activated in ABAQUS. The von Mises yield criterion is taken for the computation. Thus, yielding occurs when

$$
f\left(\sigma_{i j}\right) \equiv J_{2}-k^{2}=\frac{1}{2} s_{i j} s_{i j}-k^{2}=0
$$

where $f\left(\sigma_{i j}\right)$ denotes the von Mises yield surface, $s_{i j}$ is the stress deviator, which is related to the stress by

$s_{i j}=\sigma_{i j}-\frac{1}{3} \sigma_{k k} \delta_{i j}$

and $\mathrm{k}$ is a material parameter. Such plasticity model pertains to metallic materials, where plastic flow is governed by dislocation motion [4].

The flow rule used in the integration procedure is $\mathrm{J}_{2}$ flow theory:

$$
\dot{\varepsilon}_{i j}^{p}=\frac{3}{2} \dot{\varepsilon}_{e}^{p} \frac{s_{i j}}{\sigma_{e}}
$$

where $\dot{\varepsilon}_{i j}^{p}$ is the plastic strain rate. In accordance with Equation (3.11), the uniaxial stress-strain relations dictating the hardening response of the half space are implemented into the simulations using the von Mises equivalent stress

$$
\sigma_{e}=\sqrt{\frac{3}{2} s_{i j} s_{i j}}
$$

and effective plastic strain rate

$$
\dot{\varepsilon}_{e}^{p}=\sqrt{\frac{2}{3} \dot{\varepsilon}_{i j}^{p} \dot{\varepsilon}_{i j}^{p}}
$$

Thus, $\sigma_{e}$ is a function of $\varepsilon_{e}^{p}$ matching the uniaxial stress-plastic strain curve of the solid. 
The material is assumed to be incompressible at plastic deformation, i.e. $\dot{\varepsilon}_{k k}^{p}=0$, and thus the strain rate reduces to its deviatoric part. The equivalent strain $\varepsilon_{e}^{p}$, which characterizes the accumulated distortion of the material, is the integral

$\varepsilon_{e}^{p}=\int_{0}^{t} \dot{\varepsilon}_{e}^{p} d t$

along the deformation path.

To spare the computation source, 2-dimentional, axisymmetric models are used.

The indenter is assumed to be analytical rigid body and the specimen is deformable axisymmetric shell. Since it is axisymmetric, the real specimen it represents is a solid cylinder. The effect of the size of the indenter will be discussed in Chapter 4.

\subsubsection{Material property input}

We assume elastic power hardening plastic material model, which could be employed to describe most of engineering metals' stress-strain relationship. The elastic property includes Young's modulus E and Poisson's ration v. For plastic property, the uniaxial true stress $(\sigma)$ true plastic strain $\left(\varepsilon_{\mathrm{p}}\right)$ data are required to input by ABAQUS.

$\sigma=\left\{\begin{array}{cc}E \varepsilon & \sigma<\sigma_{Y} \\ \sigma_{y}\left(\frac{E}{\sigma_{y}}\right)^{n} \varepsilon^{n} & \text { otherwise }\end{array}\right.$

Since the power law hardening material model is used, a true stress strain data spreadsheet at first is being established by the Equation (3.3). Then use the equation: $\varepsilon_{p}=\varepsilon-\frac{\sigma}{E}$, to get plastic strain $\left(\varepsilon_{\mathrm{p}}\right)$ starting from the yield stress.

One thing to point out is the unit system in the FEM simulation. Since the ABAQUS does not designate unit system, the users could use unit system arbitrarily, as long as they are in consistency in one problem. In our problem, we are taking the force 
unit as Newton $(\mathrm{N})$ and displacement unit as micrometer $(\mu \mathrm{m})$. So the input mechanical properties value should be converted to be consistent with the units. For example, for $6061 \mathrm{~T} 6$ aluminum alloys, $\mathrm{E}=69 \mathrm{GPa}, \sigma_{\mathrm{Y}}=275 \mathrm{MPa}$. In ABAQUS, the value of Young's modulus should input as $\mathrm{E}=0.069 \mathrm{~N} / \mu \mathrm{m}^{2}$ and $\sigma_{\mathrm{y}}=275 \times 10^{-6} \mathrm{~N} / \mu \mathrm{m}^{2}$.

\subsubsection{Mesh design}

Since the indenter is much stiffer than the solids, the indenter is considered to be perfectly rigid and is modeled as analytical rigid surface. It is cost-effective since the only variables associated with a rigid surface are the translations and rotations on a single node, known as the rigid body reference node. In our case, reference point is assigned on the indenter tip, which manipulates the rigid body translation of the indenter. In addition, ABAQUS does not need to calculate the stiffness or stresses within the rigid body. The rigid surface is always the master surface in a contact pair.

Four-node axisymmetric linear quadrilateral elements are utilized. Reduced integration is employed to spare calculation time. The element type used in ABAQUS is 'CAX4R', in which the letter or number indicates the element is continuum, axisymmetric, 4-node bilinear, reduced integration with hourglass control respectively.

According to [31], 'when selecting elements for contact analysis, it is better to use first-order elements for those parts of a model which will form a slave surface. Secondorder elements can cause problems in contact simulations because of the way these elements calculate consistent nodal loads for a constant pressure.'

The mesh, which has a total of 21,573 elements, is designed so that the meshing is very fine near the indenter (in order to resolve the contact conditions and allow for accurate contact area determination), but also is sufficiently large so that it approximates 
a semi-infinite solid. Accordingly, the mesh is chosen large enough for each calculation so that the results obtained are insensitive to the movement of the outer boundaries of the mesh. The half-space solid is modeled 20 times larger than the radius of the indenter so that the boundary condition doesn't make effect on the simulation results.

To validate the convergence of the mesh, a finer mesh simulation is performed. No significant change is observed by comparing results. So the fineness of this meshing is validated. For these reasons this mesh should provides a very good approximation to an infinite solid.

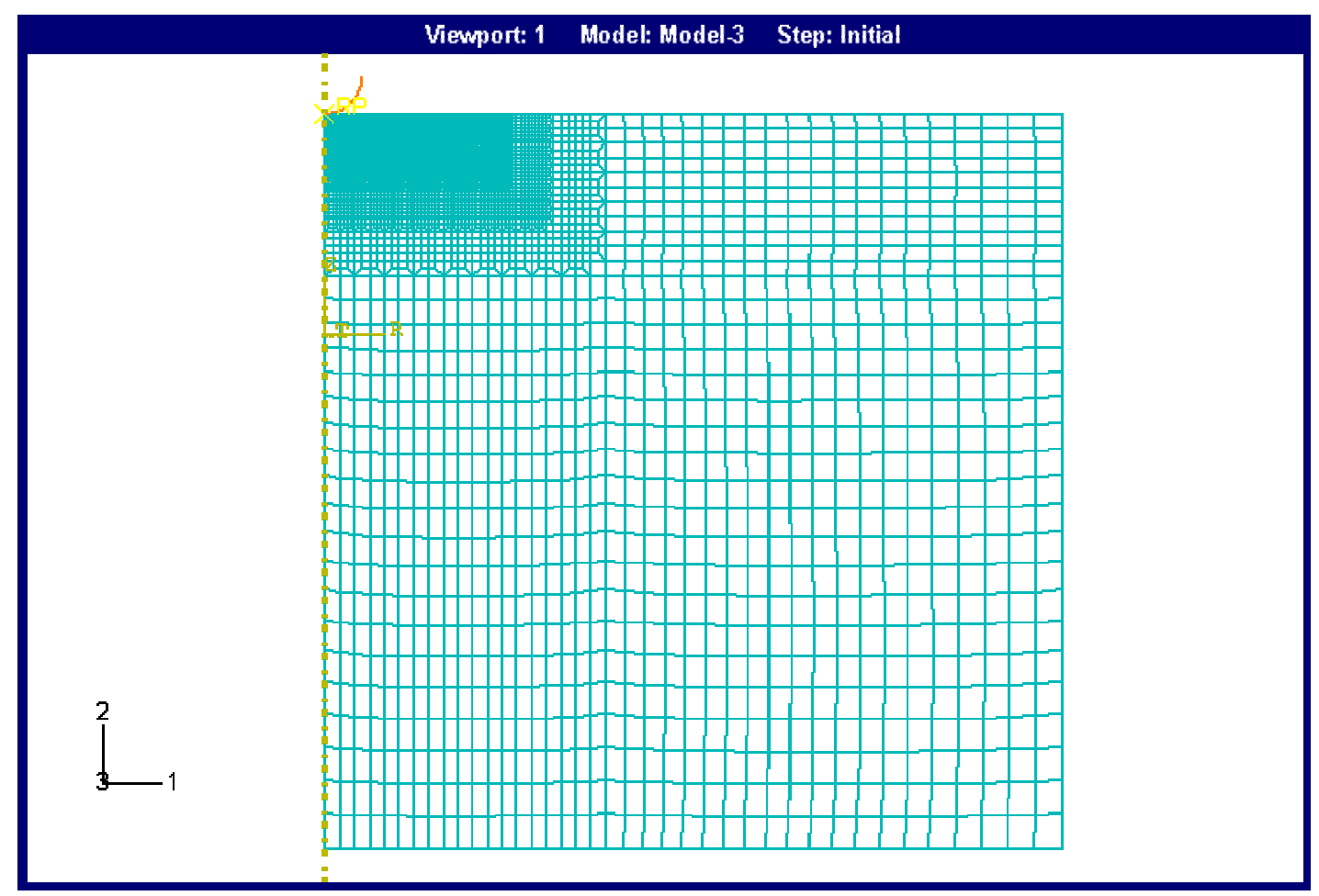

Figure 3.2 (a) Mesh design in the entire model. 


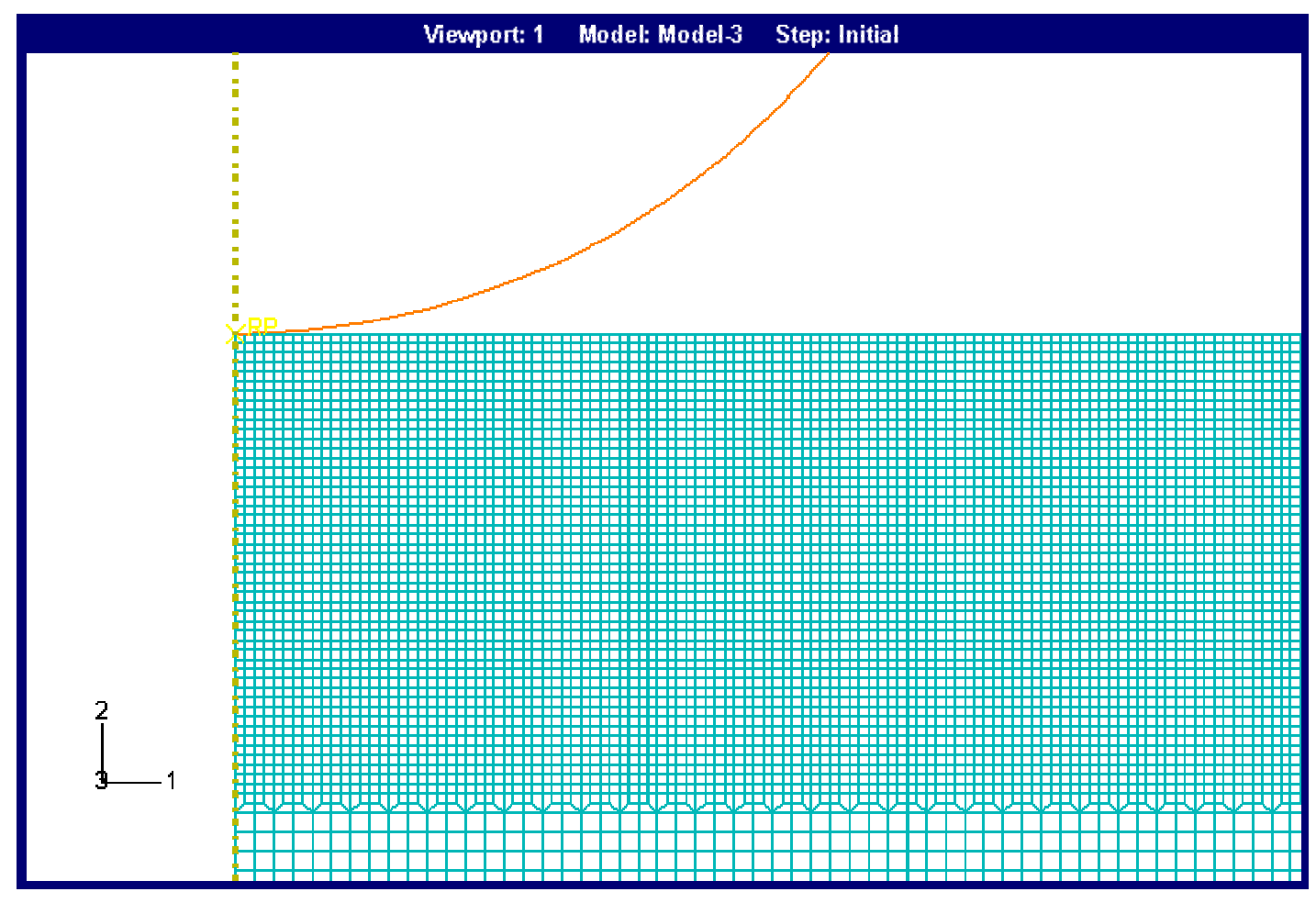

Figure 3.2 (b) Magnified view of mesh design under the sphere indenter.

Since the indentation is a localized process and the surface deformation in the area close to the indenter is of primary interest, the biased element distribution is used to save computation time while fulfill our goal. Very fine mesh is used for the area just under the indenter, and then the mesh goes coarser for the area that is farther from the indenter. The region with finer element size that fully surrounds the indenter during simulations has 10,000 elements. The presence of these very small elements on the material surface is found to have virtually no effect on the contact load calculated for a given indentation depth, but is critical in order to assure that the area is accurately calculated. A maximum of 57 elements in such region become directly in contact with the rigid spherical indenter during penetration. The trapezoid shape of element, which provides a continuum connection, is used to realize the transition from fine to coarse mesh. 
Thus, mesh design for this problem involves a delicate tradeoff between accurate resolution of the indentation zone and local behavior on one hand, and suitable representation of the far-field boundary conditions and reasonable calculation period on the other. In this study, we feel both aspects are properly handled.

\subsubsection{Boundary conditions and applying load}

To prevent the rigid body motion of the solid, it is necessary to apply boundary condition to constraint the half space solid. The bottom line is set to be fixed. The right side and top edges are free. The left side is axis of symmetry, which only allows $\mathrm{Y}$ direction translation. To verify the ratio of specimen size to indenter size is large enough so that boundary condition setup has no significant influence on the final results, another boundary condition setup is established and results are compared. Details are to be discussed in Chapter 4.

There are two methods of applying load: one is 'load control' and the other is 'displacement control'. Details are to be discussed in Chapter 4.

\subsubsection{Contact interaction}

The procedure of indenting is a typical contact problem. The interaction between contacting surfaces consists of two components: one normal to the surfaces and one tangential to the surfaces. The normal component refers to contact pressure. The tangential component consists of the relative motion (sliding) of the surfaces and,

possibly, frictional shear stresses. Coulomb friction is the model used in ABAQUS to describe the interaction of contacting surfaces. The model characterizes the friction behavior between the surfaces using a coefficient of friction $\mu$. The product $\mu \mathrm{p}$, where $\mathrm{p}$ is the contact pressure between the two surfaces, gives the limiting frictional shear stress. 
The contact surfaces will not slip (sliding relative to each other) until the shear stress across their interface equals the limiting frictional shear stress, $\mu \mathrm{p}$.

The interaction between the indenter and the specimen is modeled as contact pair. According to [31], the indenter surface is defined as the 'master' surface since the indenter is rigid body. The top of the specimen is the 'slave' surface. The friction property between them is part of the contact property and can be modified easily.

A Pentium 4, $2.2 \mathrm{GHz}$ CPU, 1 Gigabytes RAM computer under the Windows 2000 environment, completes simulations. The typical computation time for the computation is $1 \sim 2$ hours or so. 


\section{Chapter 4}

\section{Factors to Influence Results}

Before make any conclusions from FE simulation results, several influential factors are considered.

\subsection{Element selection: 'full integration' or 'reduced integration'}

'Full integration' refers to the number of Gauss points required to integrate the polynomial terms in an element's stiffness matrix exactly when the element has a regular shape. 'Reduced integration' elements use one fewer integration point in each direction than the fully integrated elements. Reduced integration, linear elements have just a single integration point located at the element's centroid. Hence, the calculation time needed by CPU is dramatically reduced.

According to [31], reduced integration elements tend to be too flexible because they suffer from severe hourglass problem. However, it is pointed out that 'as long as a reasonably fine mesh is used, that linear reduced-integration elements can give acceptable results'. Since fine mesh is put near the indenter, the hourglass effect should be eliminated in this simulation.

To make sure the hourglass effect is limited, 'full integration' elements (CAX4) is used to replace current 'reduced integration' elements (CAX4R). No significant difference in result comparison is observed. So, the reduced integration elements are used in following simulations.

\subsection{Two methods of applying load: 'load control' and 'displacement control'}

Two methods could be applied to press the indenter into half-space material: load control and displacement control. 
When the load is controlled, the concentrated force is applied on the analytical rigid indenter. Due to the nonlinearity of this finite element analysis, the final load is applied through incremental steps. Total time increment is 1 . For each increment, use the linear proportion to calculate real load P. The indentation depth (hs) is extracted by observing vertical displacement of node which is just below the indenter. Thus the loaddepth curve could be established.

When using displacement control method, the vertical displacement of the rigid indenter is set as input, which is also corresponding to indentation depth. For each increment, the indentation depth (hs) is calculated by linear proportion to the incremental time. The real load (P) applied on the indenter is the summation of $\mathrm{Y}$ reflection force on the bottom line of the half space. The P-hs curve is thus obtained.

Two simulations based on these two methods respectively are performed to observe the influence. The material is selected as Inconel 783 super alloy, which has the material mechanical properties as below:

$$
\begin{aligned}
& \mathrm{E}=177.3 \mathrm{GPa}, \nu=0.3 \\
& \sigma_{\mathrm{y}}=779 \mathrm{MPa} \\
& \mathrm{n}=0.107
\end{aligned}
$$

Also the same coefficient of friction for contact interaction between indenter and half space surface is used in both simulations. The spherical indenter which has diameter of $1.6 \mathrm{~mm}$ is simulated. The load-displacement curves (P-hs) are extracted respectively from both simulations and results are plotted in Excel. 
P-hs curve comparison

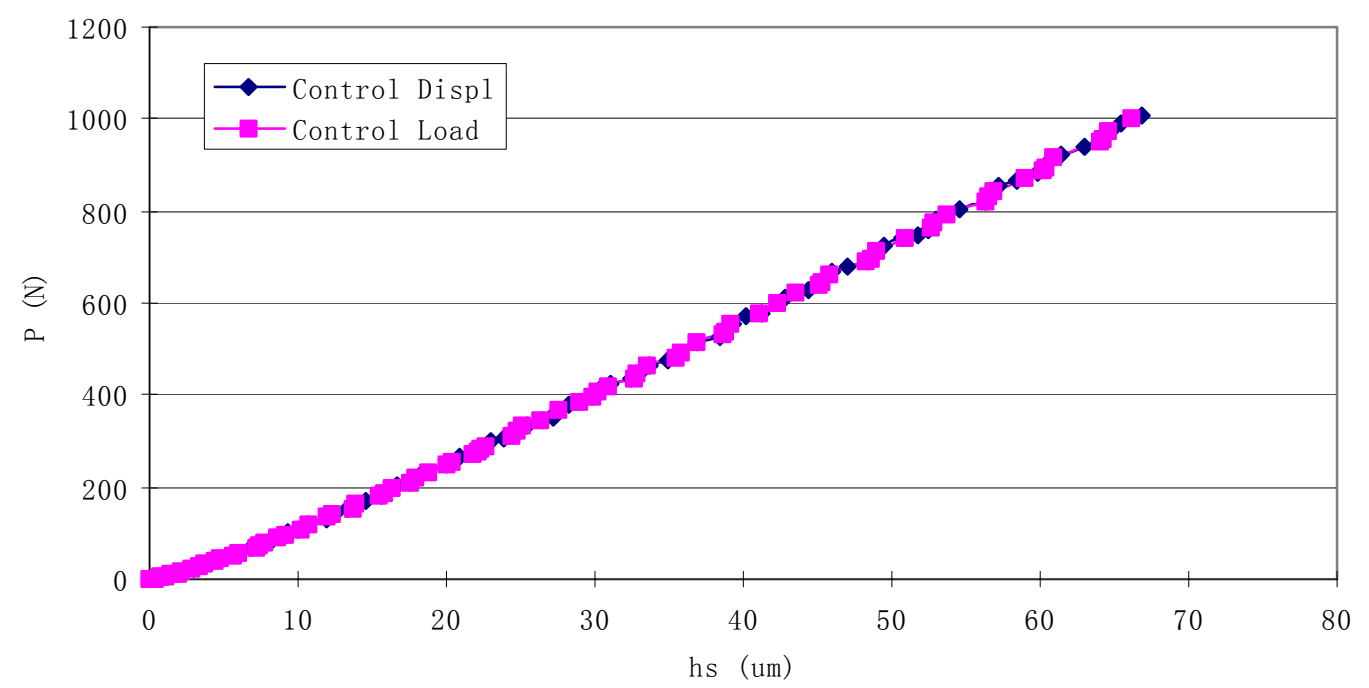

Figure 4.1 Load-depth curve comparisons for different loading methods.

The comparison shows the two curves overlapped each other very well, which indicates the results are essentially the same no matter which method of applying load is used. In the subsequent simulations, the 'control displacement' method is to be employed if no special indication.

\subsection{Boundary condition effect}

As discussed in Chapter 3, to evaluate whether the specimen dimensions are large enough to approximate the behavior of a semi infinite half-space, two different boundary conditions are applied in the modeling.
A: Roller side, roller bottom
B: Free side, fixed bottom 


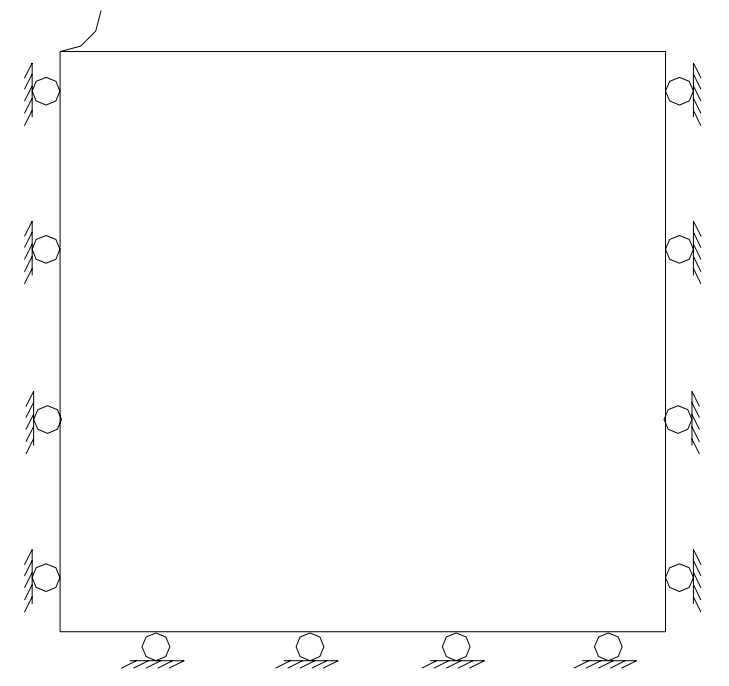

Figure 4.2 (a) Scheme of (A) boundary condition setup (roller bottom, roller side).

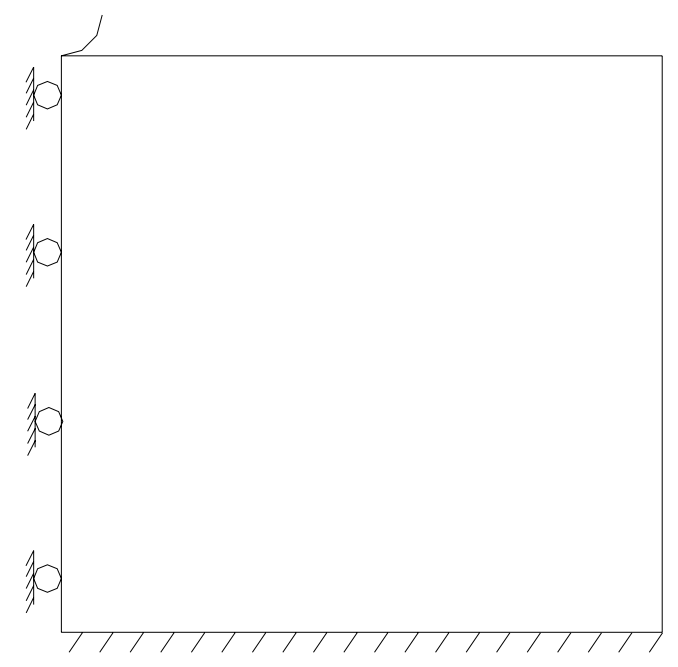

Figure 4.2 (b) Scheme of (B) boundary condition setup (fixed bottom, free side).

In both simulations, the same material, AL6061 T6, is selected to perform. The mechanical properties of this material are: $\mathrm{E}=69 \mathrm{GPa}, \mathrm{v}=0.33, \sigma_{\mathrm{y}}=275 \mathrm{MPa}, \mathrm{n}=0.09$. Spherical indenter with diameter $\mathrm{D}=1.6 \mathrm{~mm}$ is used. Results of both load-depth curve and surface contour are compared. 


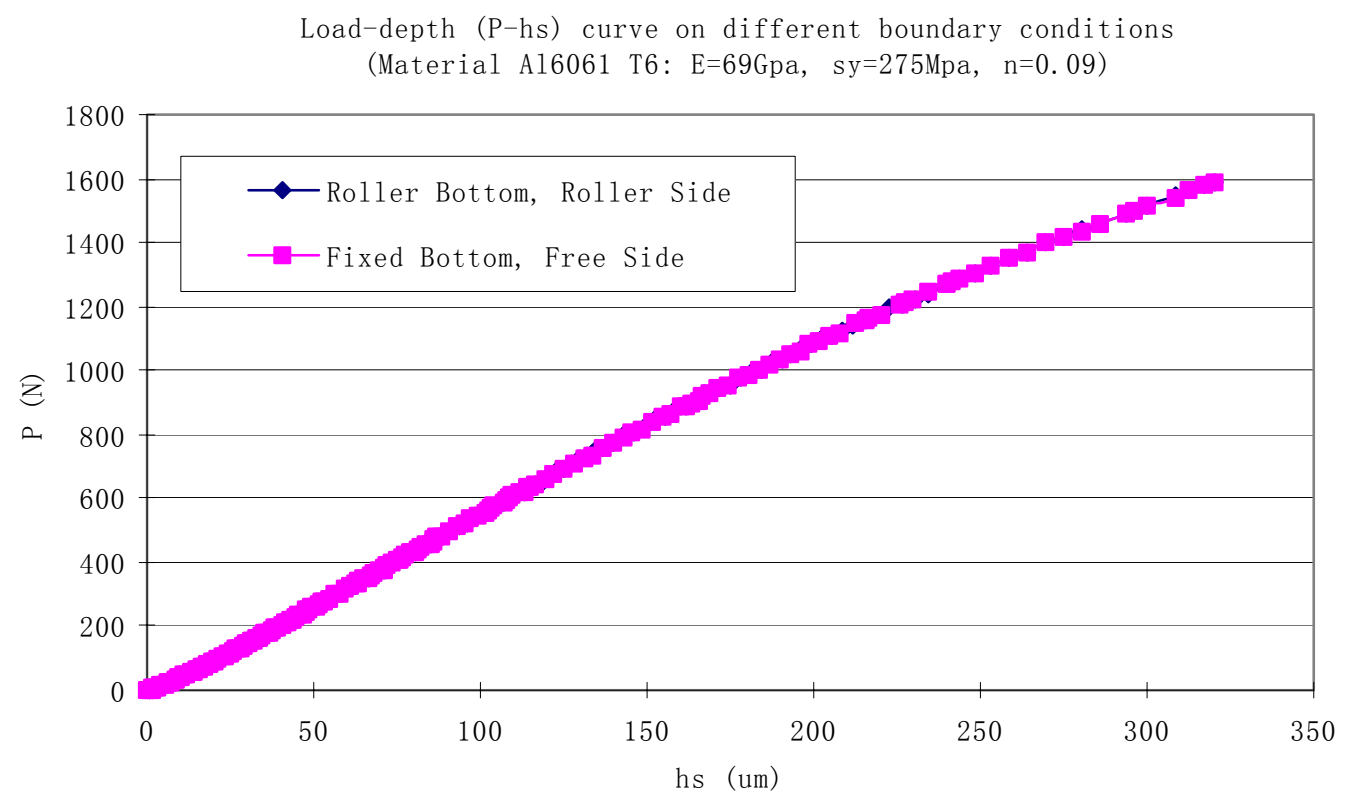

Figure 4.3 (a) Load-depth curve for different boundary conditions.

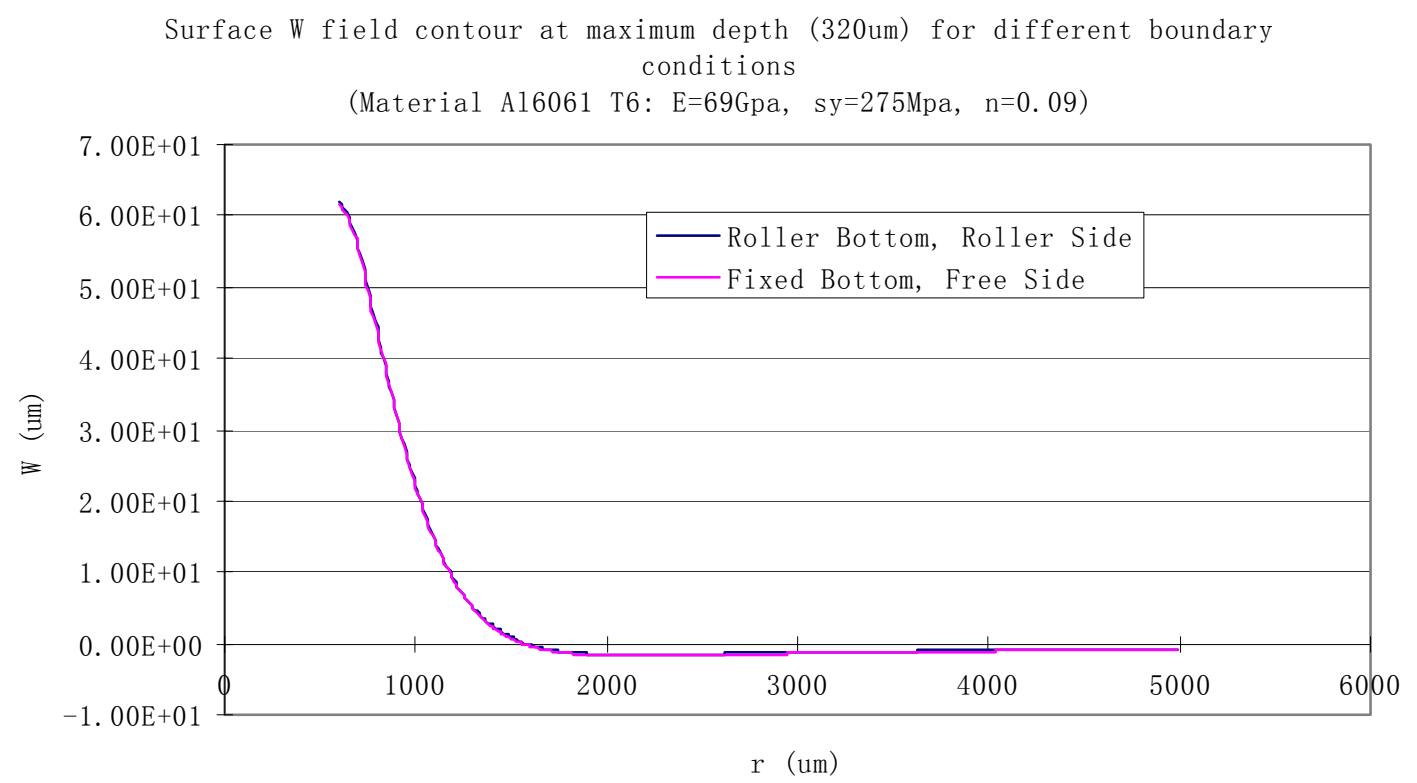

Figure 4.3 (b) Surface W field contour for different boundary conditions.

The results indicated that the ratio of specimen size to the indenter size is large enough and boundary condition has negligible effect on the simulation results. In 
subsequent simulations, the B boundary conditions (fixed bottom, free side) are employed.

\subsection{Coefficient of friction effect}

Special attention is paid to the friction coefficient effect between the indenter and the specimen surface. In earlier research, the indenter- specimen interaction property is usually set to be frictionless. In Nix's simulation [10,11], 'the interface between the specimen and the indenter was assumed to be frictionless since no noticeable change in the load-displacement response was observed by using a friction coefficient of $1 . '$ Although Bower et al. [35] and Komvopoulos [36] may lead to disregard the influence of friction on the contact response; further analysis by Mesarovic and Fleck [37] showed that contact area could decrease by $8 \%$ for the extreme case of a sticking spherical indenter. Vasinonta and Beuth [38] claimed the friction coefficient used in the modeling could considerably affect the numerical results.

In our research, the surface deformation is the main concern, which will be greatly affected by the friction between the indenter and the specimen. Four different friction simulations are performed to compare the results, which are

1) Frictionless $(\mu=0)$

2) Friction coefficient $\mu=0.1$

3) Friction coefficient $\mu=0.2$

4) Rough contact $(\mu=\infty$, which means there cannot have relative sliding movement once the indenter is contacted with the specimen surface)

The typical material, Aluminum $6061 \mathrm{~T} 6$ is analyzed here. The original uniaxial tensile test data is given in Chapter 3, Figure 3.1. The material mechanical properties are: 
$\mathrm{E}=69 \mathrm{GPa}, v=0.33, \sigma_{\mathrm{y}}=275 \mathrm{MPa}, \mathrm{n}=0.09$. The ball indentation with diameter $\mathrm{D}=0.1 \mathrm{~mm}$ is simulated.

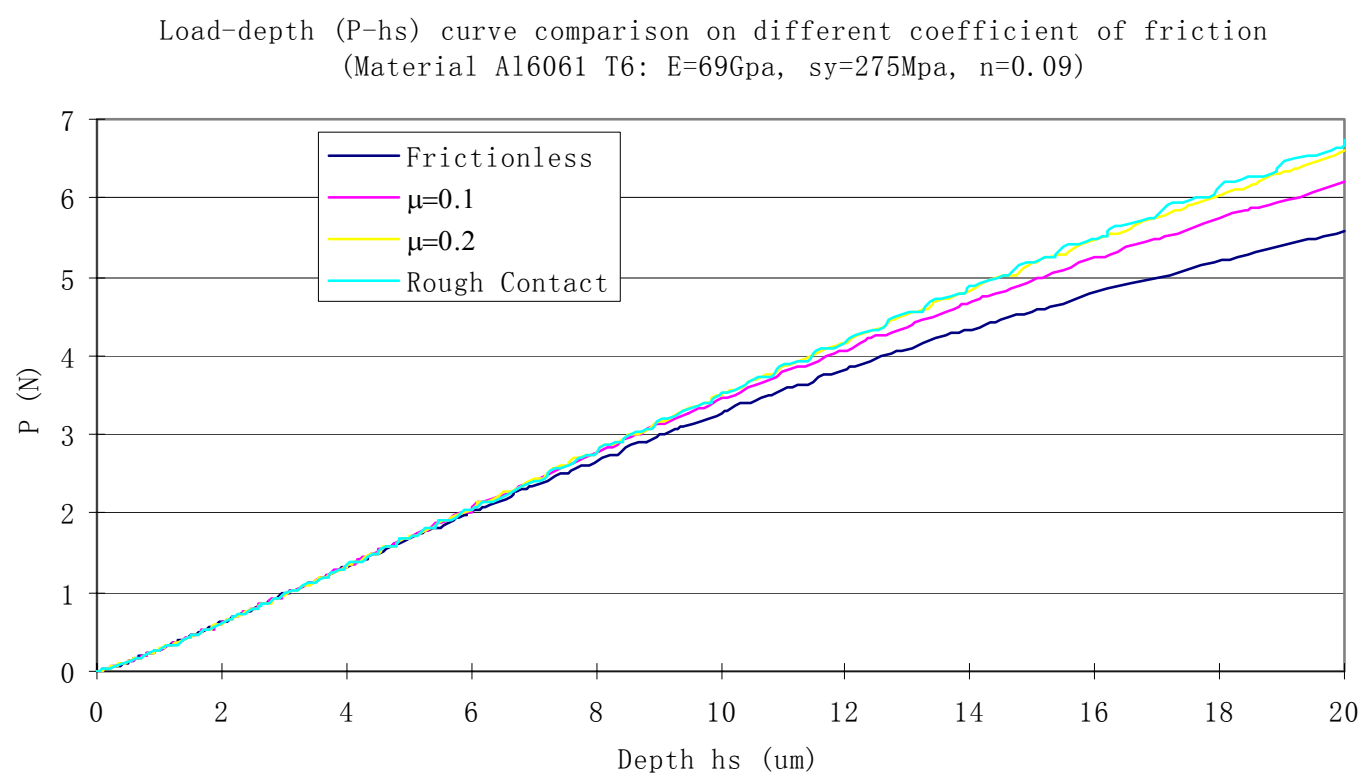

Figure 4.4 (a) Load-depth curve comparisons for different coefficient of friction.

Figure 4.4 (a) indicates that the P-hs curve would get higher with the increase of the friction coefficient, which is consistent with the simulation results of Aditad Vasinonta and Jack Beuth [38] for cone indentation. 
The effect of friction coefficient on $W$ distribution

(Material: $\mathrm{E}=69$ GPa $\sigma_{\mathrm{y}}=275 \mathrm{MPa} \mathrm{n}=0.09$ )

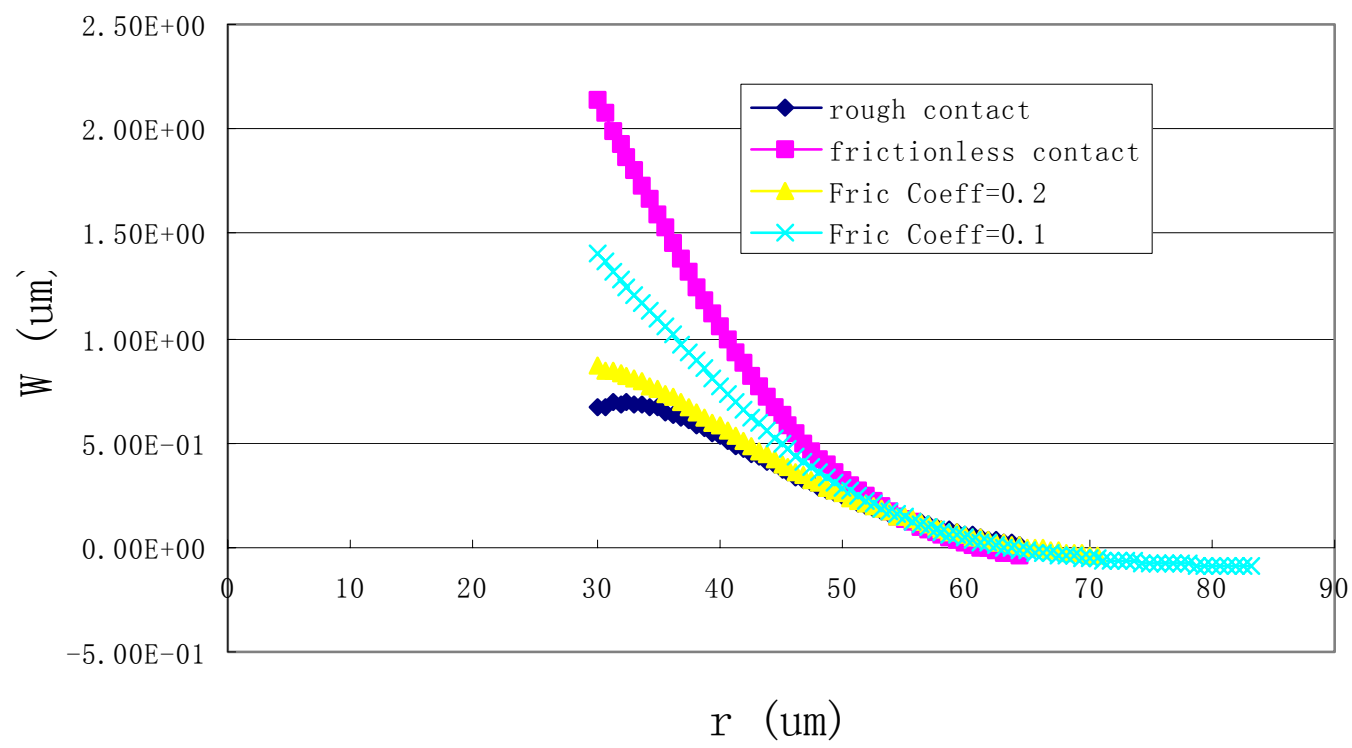

Figure 4.4 (b) W distributions for the same material but different friction property.

(All the 4 cases have the same indentation depth $\mathrm{hs}=10 \mu \mathrm{m}$ for $\mathrm{D}=0.1 \mu \mathrm{m}$ ball indenter)

Figure 4.4 (b) indicates the $\mathrm{W}$ field distribution along the radial direction at the same indentation depth for the four kinds of friction property. As shown in the graph, it is concluded that the friction property has significant effect on the surface deformation for the area that is very close to the indenter. The influence becomes less when the observed area is far away from the indenter.

It shows that the friction coefficient used in the modeling can considerably affect the numerical results, both load-depth curve and the surface displacement contour. Thus inclusion of frictional effects is typically important in modeling indentation. According to the reference [34], the static friction coefficient between the diamond and metal is usually $0.1 \sim 0.15$. And the dynamic friction coefficient is smaller than the static one. So, we chose friction coefficient $\mu=0.1$ for the subsequent simulations. 


\subsection{Indenter size effect}

To assess the indenter size effect on the simulation results, two simulations are performed. The diameter of the spherical indenter are $\mathrm{D}=0.1 \mathrm{~mm}$ and $\mathrm{D}=1.6 \mathrm{~mm}$ (Rockwell B) respectively. The size of the half space specimen is modeled as $1 \mathrm{~mm} \times 1 \mathrm{~mm}$ and $16 \mathrm{~mm} \times 16 \mathrm{~mm}$ correspondingly.

The same material and friction characteristics are employed for both simulations. The selected material is Al6061 T6 with mechanical properties as $\mathrm{E}=69 \mathrm{GPa}, \mathrm{v}=0.33$, $\sigma_{\mathrm{y}}=275 \mathrm{MPa}, \mathrm{n}=0.09$, coefficient of friction $\mu=0.1$

Surface contour distribution at max load for different size of indenter comparison

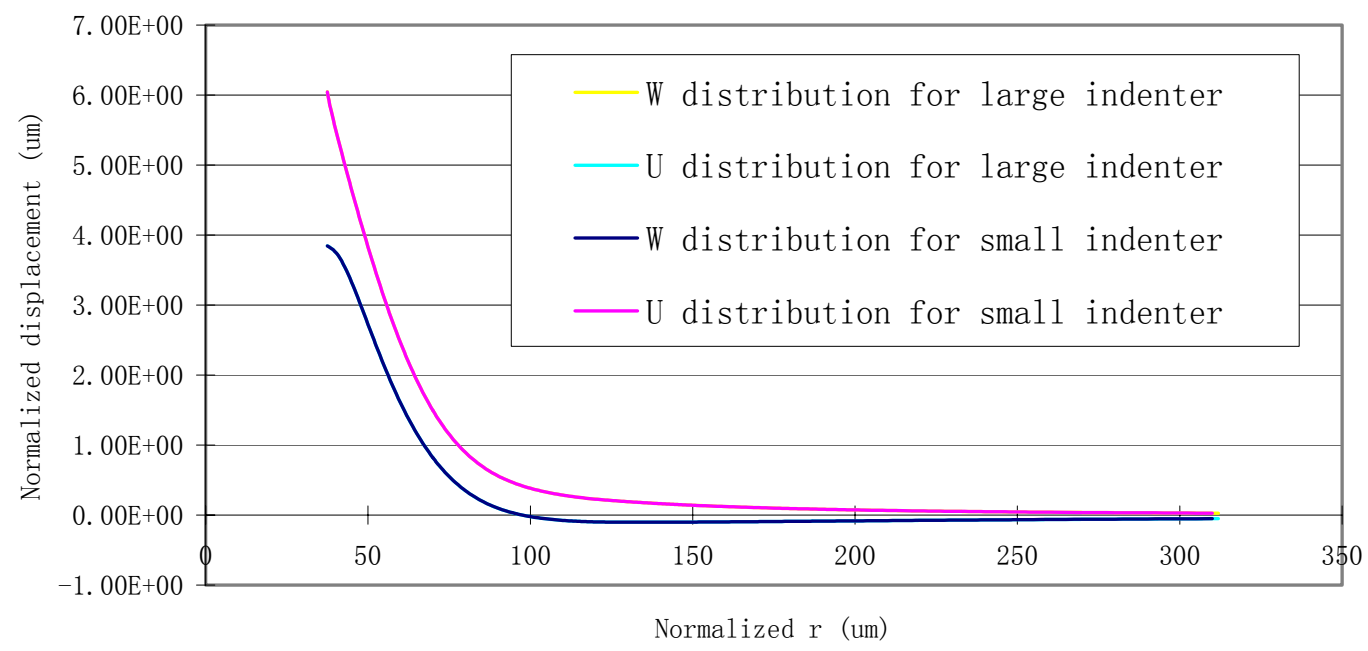

Figure 4.5 Normalized surface displacement distribution for different indenter size.

Simulation results are extracted out at the same loading stage (e.g., maximum loading depth, which is $1 / 5$ of the indenter diameter). In Figure 4.5, normalized $r$ is the real location for small indenter and real location divided by 16 for large indenter. The same definition for normalized displacement. It is indicated in Figure 4.5 that both normalized $\mathrm{U}$ and $\mathrm{W}$ displacement values on all surface nodes overlapped perfectly when 
comparing the case 1 and case 2 simulation results. So conclusively, the indenter size effect on displacement fields is characteristic of self-similarity.

von Mises stress distribution for different indenter size

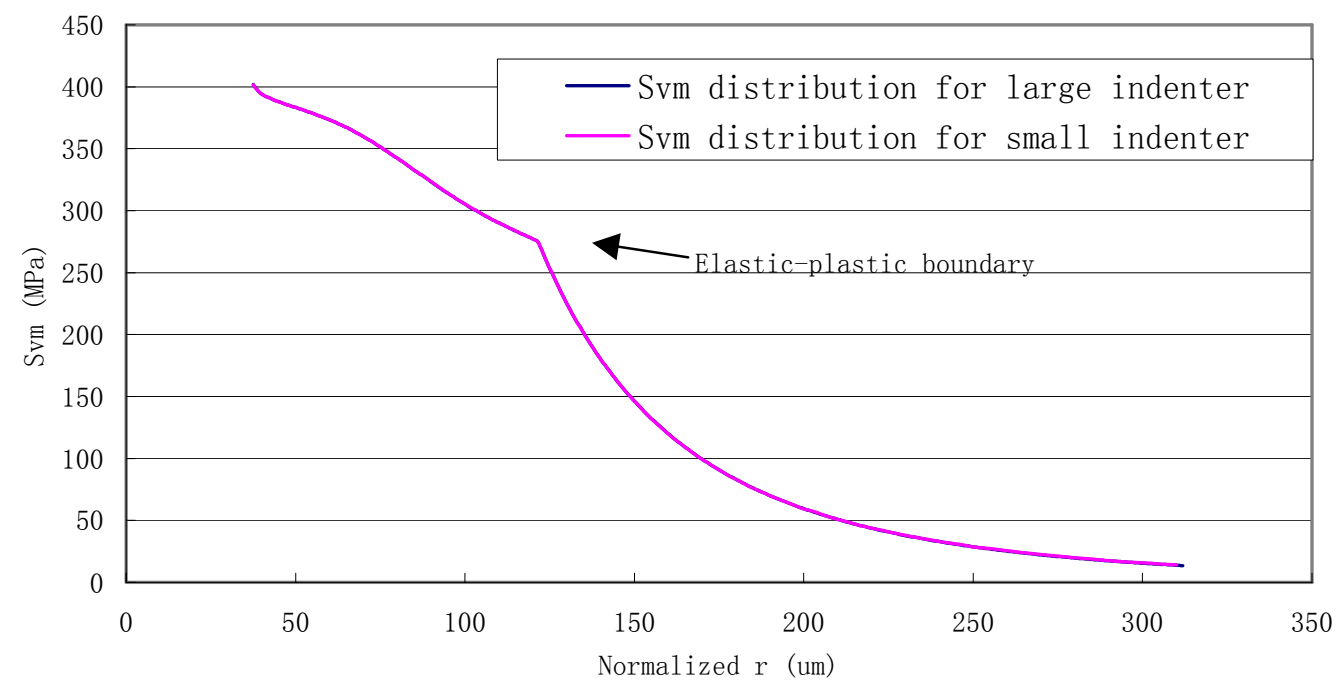

Figure 4.6 Normalized surface von Mises distribution for different indenter size.

The self-similarity characteristics also exist in the von Mises stress distribution, which is shown in Figure 4.6. Since the elastic-plastic boundary is distinguished by the yield stress, which is von Mises effective stress in multidimensional state of stress, the EP boundary is also self-similar. 


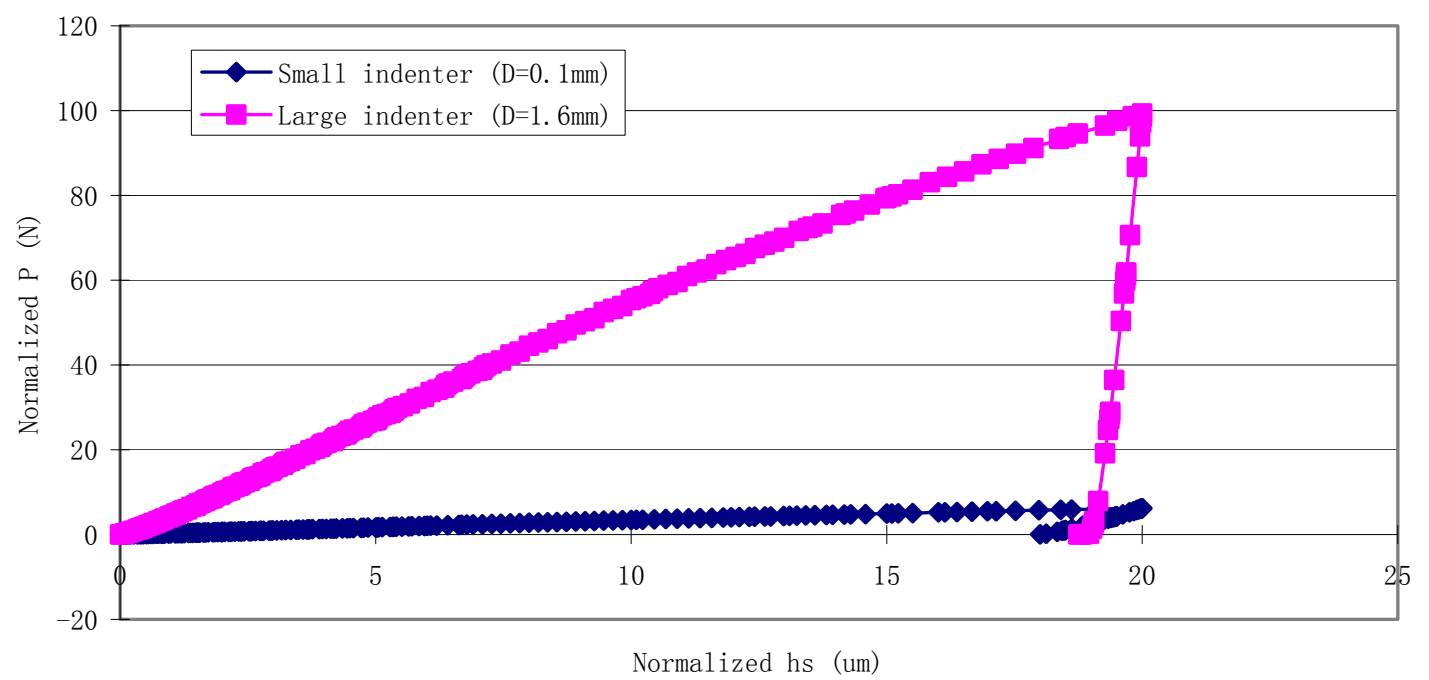

Figure 4.7 Normalized load depth curve for different indenter size.

Similar graph is plotted for the load-depth curve, shown in Figure 4.7. When checking the force applied on the indenter to make the same proportion of depth $(1 / 5$ of the indenter diameter), the self-similarity characteristics does not exist. For large indenter, the normalized force needed to press the indenter to the same normalized depth as small indenter needed is much larger. The increase of the size of the indenter will increase the load significantly to achieve the same normalized depth. 


\section{Chapter 5}

\section{Verification of the Finite Element Model}

\subsection{Verification by comparison with elastic analytical results}

To examine the suitability of the current finite element mesh and modeling assumptions, elastic indentation simulations are performed and the solutions for the surface stresses and displacements are compared with results from the elastic analysis of Hertz.

\subsubsection{Hertz theory of elastic indentation}

Hertz was concerned with the nature of the localized deformation and the distribution of pressure between two elastic bodies placed in mutual contact. He sought to assign a shape to the surface of contact that satisfied certain boundary conditions, namely:

i. The displacements and stresses must satisfy the differential equations of equilibrium for elastic bodies and the stresses must vanish at a great distance from the contact surface.

ii. The bodies are in frictionless contact.

iii. At the surface of the bodies, the normal pressure is zero outside and equal and opposite inside the circle of contact.

iv. The distance between the surfaces of the two bodies is zero inside and greater than zero outside the circle of contact.

v. The integral of the pressure distribution within the circle of contact with respect to the area of the circle of contact gives the force acting between the two bodies. 
These conditions define a framework within which a mathematical treatment of the problem may be formulated.

\subsubsection{Surface stress and displacement distribution}

The normal pressure distribution directly beneath a spherical indenter was given by Hertz:

$$
\frac{\sigma_{z}}{p_{m}}=-\frac{3}{2}\left(1-\frac{r^{2}}{a^{2}}\right)^{1 / 2} \quad r \leq a
$$

From the above equation, $\sigma_{z}=1.5 p_{m}$ is a maximum at the center of contact and is zero at the edge of the contact circle. Outside the contact circle, the normal stress $\sigma_{z}$ is zero, it being a free surface.

The out-of-plane displacement of points on the surface of the specimen within the contact circle, measured with respect to the original specimen free surface, is:

$$
u_{z}=\frac{1-v^{2}}{E} \frac{3}{2} p_{m} \frac{\pi}{4 a}\left(2 a^{2}-r^{2}\right) \quad r \leq a
$$

and outside the contact circle is:

$$
u_{z}=\frac{1-v^{2}}{E} \frac{3}{2} p_{m} \frac{1}{2 a}\left[\left(2 a^{2}-r^{2}\right) \sin ^{-1} \frac{a}{r}+a r\left(1-\frac{a^{2}}{r^{2}}\right)^{1 / 2}\right] \quad r \geq a
$$

Equation (5.3) shows that the depth beneath the original surface of the contact circle (at $r / a=1$ ) is exactly one-half of the total depth at $r=0$, which indicates the surface deformation mode is always sinking-in for pure elastic materials.

Inside the contact circle, the radial stress distribution at the surface is:

$$
\frac{\sigma_{r}}{p_{m}}=\frac{1-2 v}{2} \frac{a^{2}}{r^{2}}\left[1-\left(1-\frac{r^{2}}{a^{2}}\right)^{3 / 2}\right]-\frac{3}{2}\left(1-\frac{r^{2}}{a^{2}}\right)^{1 / 2} \quad r \leq a
$$


and on the surface outside the contact circle:

$$
\frac{\sigma_{r}}{p_{m}}=\frac{1-2 v}{2} \frac{a^{2}}{r^{2}} \quad r>a
$$

The maximum value of $\sigma_{r}$ occurs at $\mathrm{r}=\mathrm{a}$.

In-plane displacements on the surface beneath the indenter in the radial direction are given by:

$$
u_{r}=-\frac{(1-2 v)(1+v)}{3 E} \frac{a^{2}}{r} \frac{3}{2} p_{m}\left[1-\left(1-\frac{r^{2}}{a^{2}}\right)^{3 / 2}\right] \quad r \leq a
$$

Note that for all values of $r<a$, the radial displacement of points on the surface is inward toward the center of contact. Outside the contact area, the radial displacements are given by:

$u_{r}=-\frac{(1-2 v)(1+v)}{3 E} \frac{a^{2}}{r} \frac{3}{2} p_{m} \quad r>a$

The hoop stress, on the surface, is always a principal stress and outside the contact circle is equal in magnitude to the radial stress:

$$
\sigma_{\theta}=-\sigma_{r} \quad r>a
$$

The in-plane displacements on the hoop direction are always zero due to the axisymmetric characteristics of the problem.

\subsubsection{Load depth (P-hs) relationship}

A general theoretical framework can be proposed in terms of the original formulation derived by Hertz. In the context of P-hs measurements on a flat surface (with infinite radius of curvature), which is indented by an elastic sphere, Hertz showed that: 


$$
P=C h_{s}^{3 / 2}, \quad C=\frac{2 \sqrt{2}}{3} E_{r} D^{1 / 2}
$$

where $\mathrm{D}$ is the diameter of the sphere and Er is the reduced Young's modulus of the specimen-indenter system defined by Equation (2.6).

When the spherical indenter is assumed to be perfectly rigid, the Equation (5.8) deforms to be:

$$
P=\frac{2 \sqrt{2}}{3} \cdot \frac{E \sqrt{D}}{1-v^{2}} h_{s}^{3 / 2}
$$

\subsubsection{Elastic finite element model}

In the elastic finite element model, the mesh discussed in Chapter 3 is employed. Frictionless interface is assumed to compare results with Hertz analytical solution. Only elastic properties are required to be input in ABAQUS. Here $E=69 \mathrm{MPa}$ and $v=0.33$ are used.

Since the analytical elastic solution is based on the assumption of small deformation, a relatively shallow indentation is simulated to compare with it. The maximum indentation depth $\mathrm{hs}=10 \mathrm{um}$, using diameter $\mathrm{D}=1.6 \mathrm{~mm}$ indenter. Also the indenter here is assumed to be perfectly rigid. 


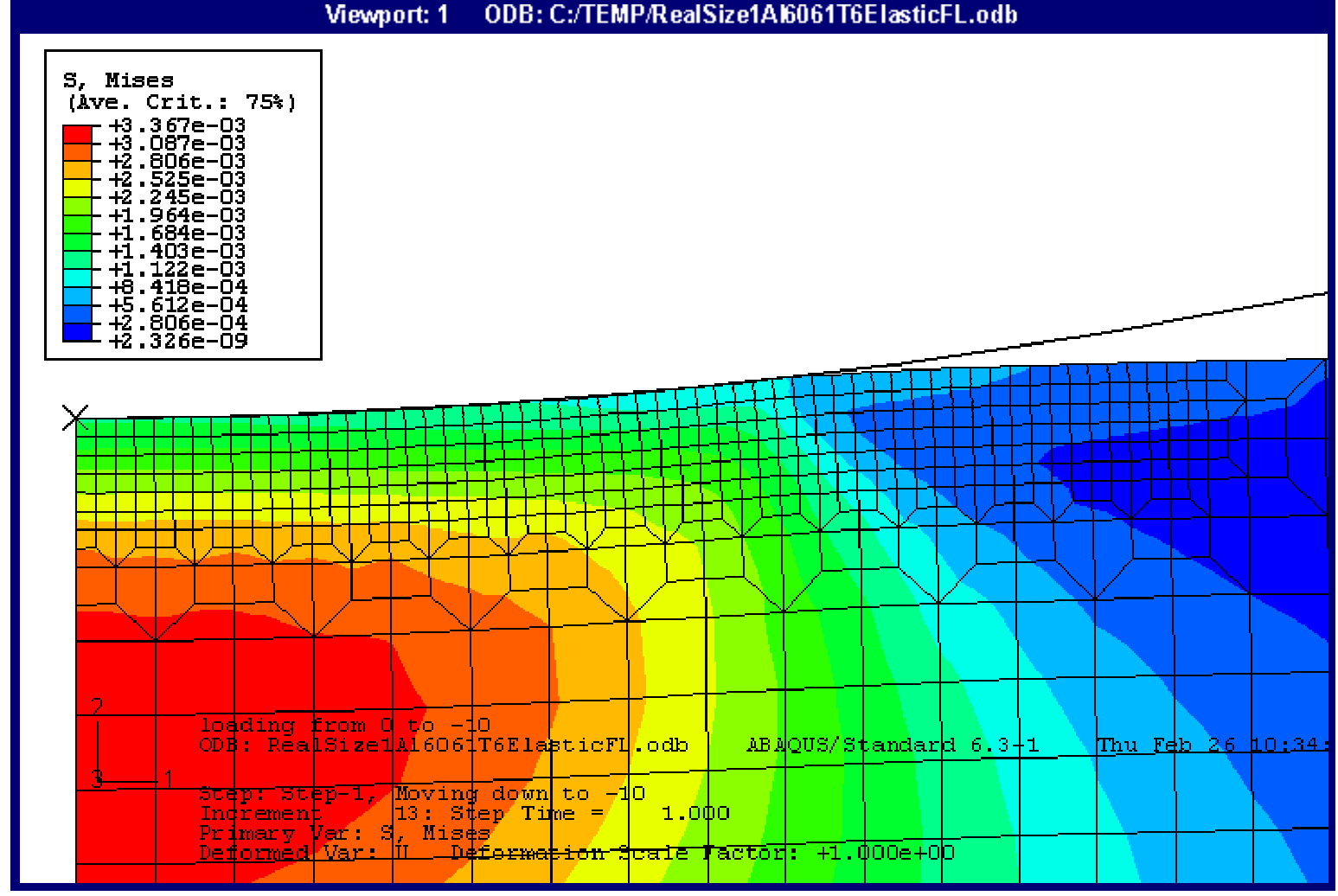

Figure 5.1 von Mises stress contour plot beneath the rigid spherical indenter.

From the FEM results, it is obtained that:

Maximum load $P_{\max }=93.4915 N$

Contact radius $a=89.5 \mu m$

So the contact pressure could be calculated by:

$$
p_{m}=\frac{P_{\max }}{A}=\frac{P_{\max }}{\pi a^{2}}=3715.15 M P a
$$

Also the nodal $\mathrm{W}\left(\mathrm{u}_{\mathrm{z}}\right)$ and $\mathrm{U}\left(\mathrm{u}_{\mathrm{r}}\right)$ displacements, $\sigma_{\mathrm{z}}$ and $\sigma_{\mathrm{r}}$ on the specimen surface are extracted out to compare with the Hertz analytical solution.

\subsubsection{Comparison between FEM results and analytical solution}


W $\left(\mathrm{u}_{\mathrm{z}}\right)$ field comparison between FEM and analytical results

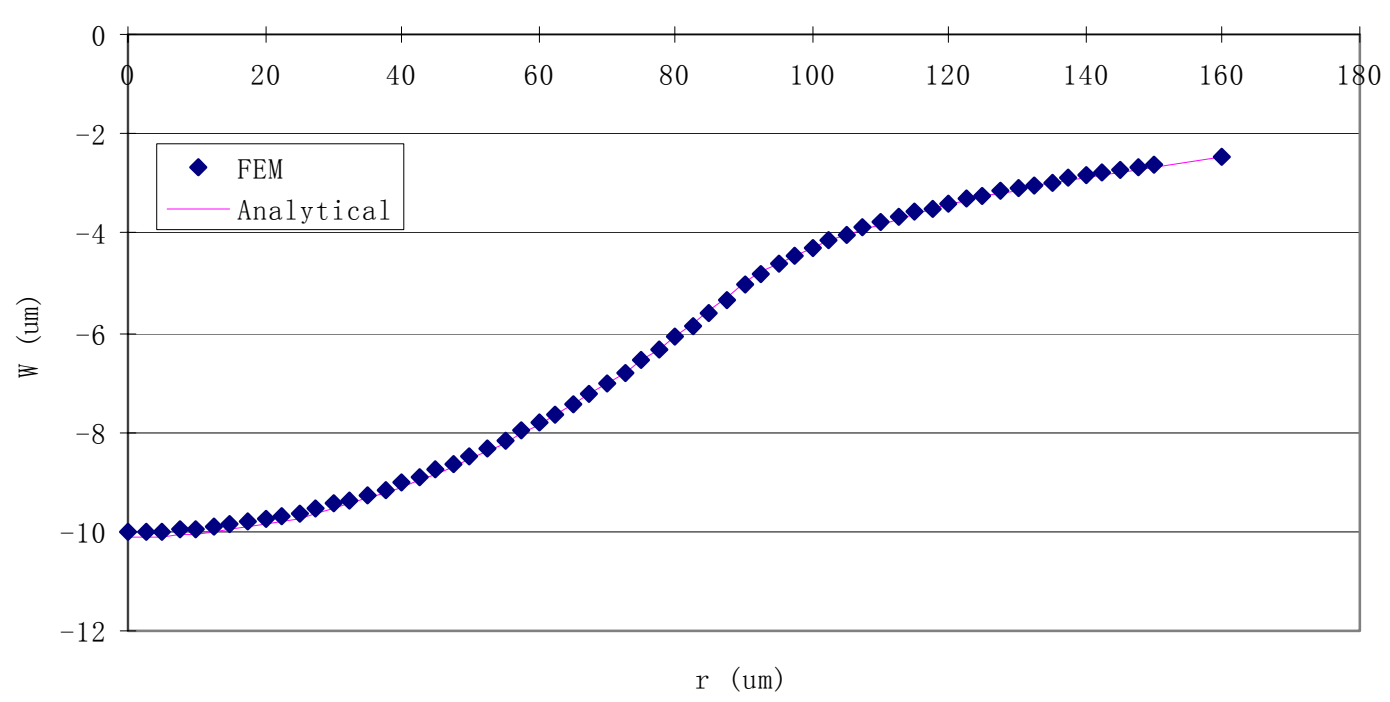

Figure $5.2(\mathrm{a}) \mathrm{W}\left(\mathrm{u}_{\mathrm{z}}\right)$ field comparison between FEM and analytical results.

$\sigma_{\mathrm{z}}$ comparison between FEM and Analytical results

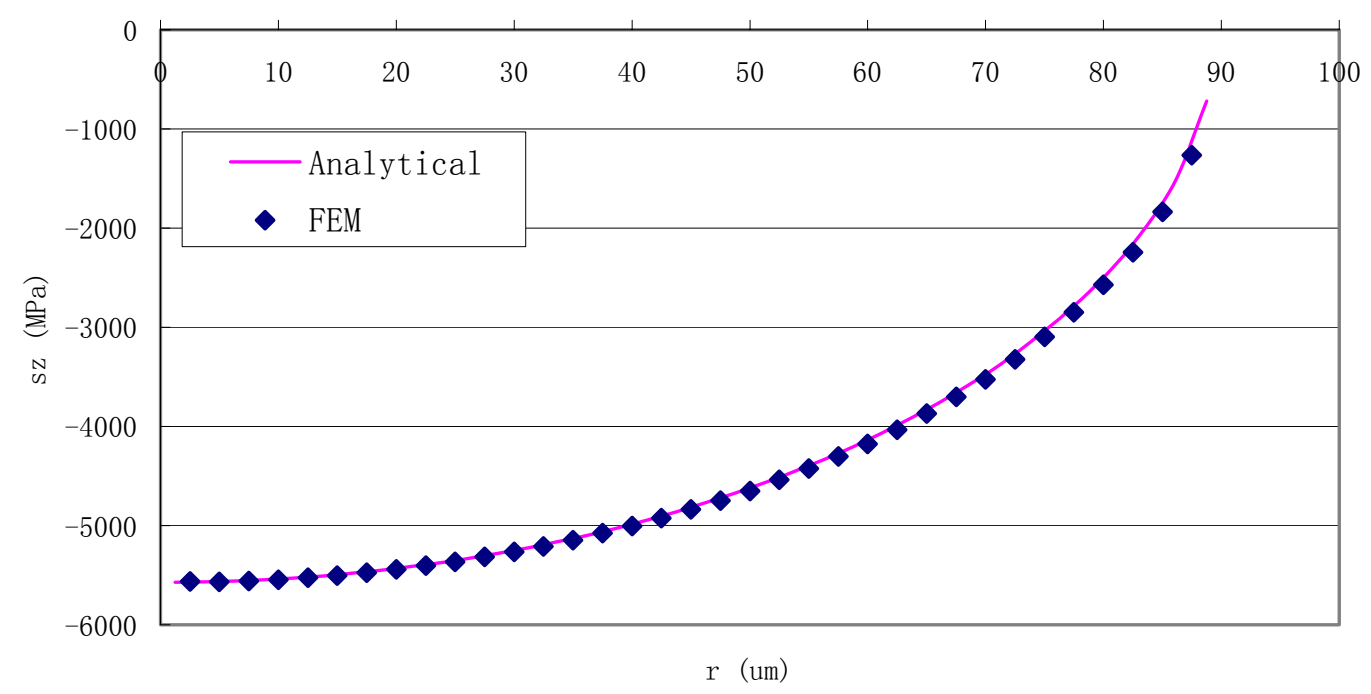

Figure $5.2\left(\right.$ b) $\sigma_{z}$ comparison between FEM and analytical results. 
$\mathrm{U}\left(\mathrm{u}_{\mathrm{r}}\right)$ comparison between FEM and analytical results

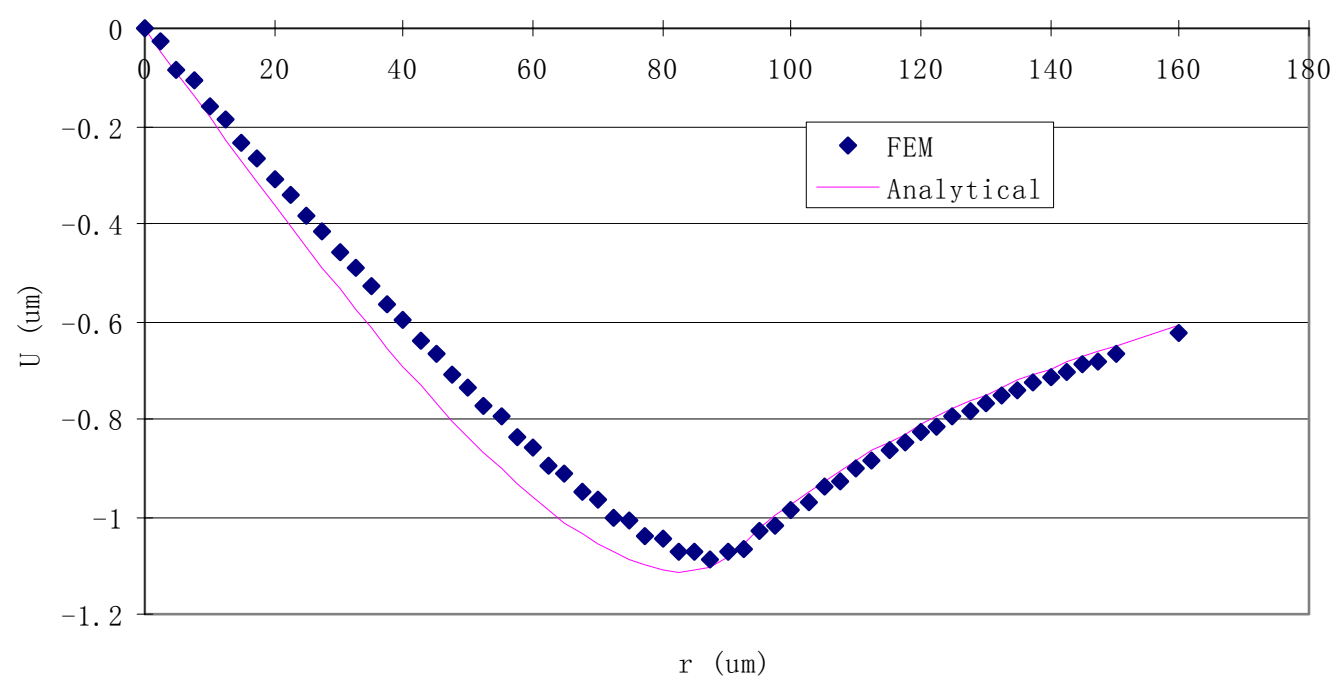

Figure 5.3 (a) $\mathrm{U}\left(\mathrm{u}_{\mathrm{r}}\right)$ field comparison between FEM and analytical results.

$\sigma_{\mathrm{r}}$ comparison between FEM (node) and analytical results

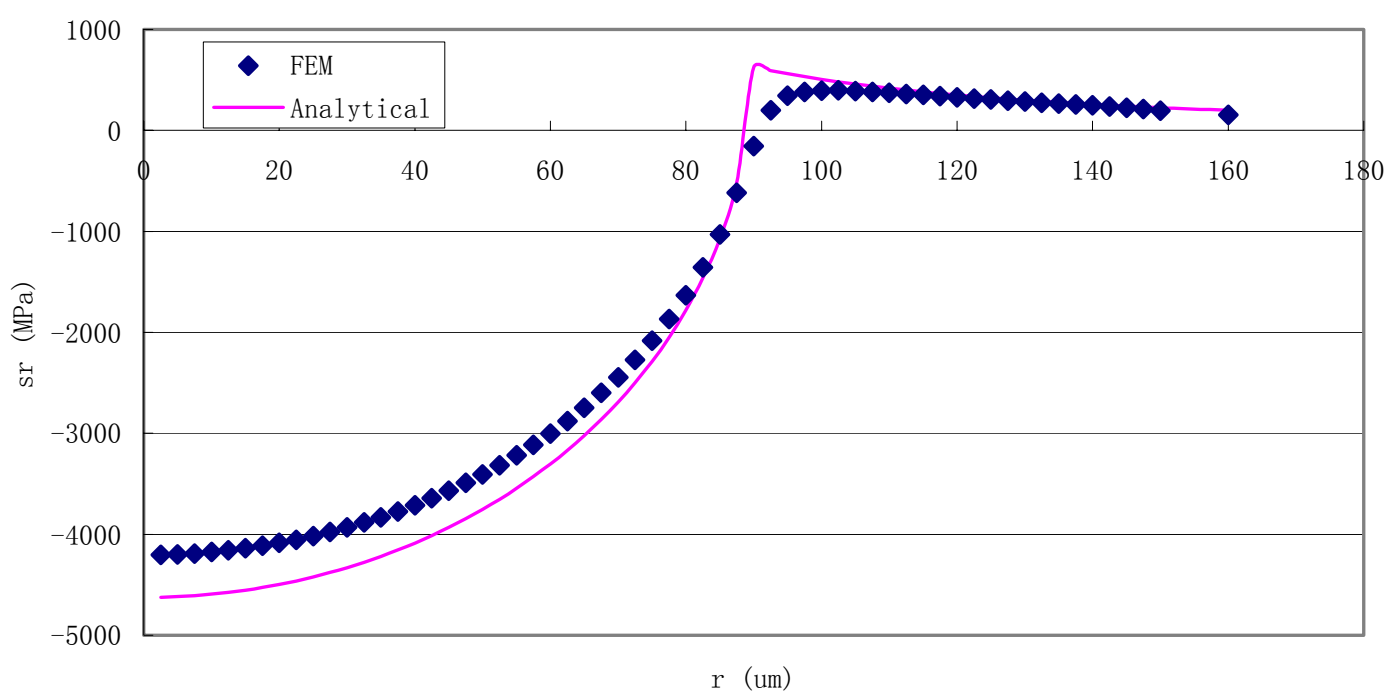

Figure $5.3(\mathrm{~b}) \sigma_{\mathrm{r}}$ comparison between FEM and analytical results. 
Load depth curve comparison between FEM and analytical solution

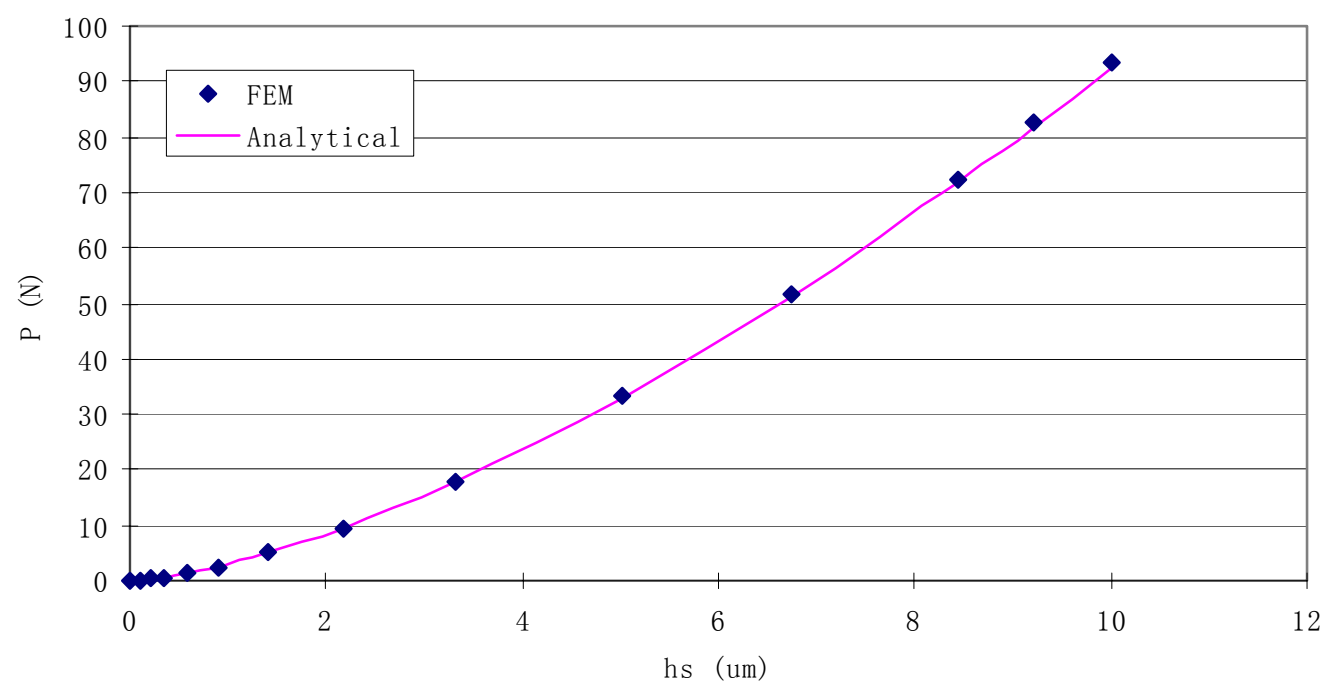

Figure 5.4 Load depth curve comparison between FEM and analytical results.

The figures indicate the agreement between finite element and analytical results is appreciably good, especially for the $\mathrm{W}\left(\mathrm{u}_{\mathrm{z}}\right)$ displacements and $\sigma_{\mathrm{z}}$ stress comparisons and load depth curve comparison. The small errors in the comparison of $\mathrm{U}\left(\mathrm{u}_{\mathrm{r}}\right)$ displacements and $\sigma_{\mathrm{r}}$ stress can be attributed to the discretization of the continuous surface and averaging of the stress data from the integration points to boundary nodes, which do not have elements on all sides.

It is also noticed that the agreement of load-depth curve fits better in the initial load than the large load. The latter part error on the comparison curve could result from the large deformation underneath the indenter caused by large load, which is not compatible with the small deformation assumption of elastic theory.

In view of the favorable comparisons with Hertz analytical results, it may be concluded that the finite element mesh and modeling assumptions are appropriate for simulating the indentation of a half-space by a rigid sphere. 


\subsection{Experimental verification}

Real ball indentation experiments are performed on Inconel 783 Alloy, made by Special Metals Company. The material mechanical properties are obtained from [57], which are $\mathrm{E}=177.3 \mathrm{GPa}, \nu=0.31, \sigma_{\mathrm{y}}=779 \mathrm{MPa}$. From the uniaxial tensile test on this material, the stress strain curve behaves double linear relationship. It is given $\sigma_{\mathrm{ult}}=1194$ $\mathrm{MPa}$ at 20\% elongation in [57].

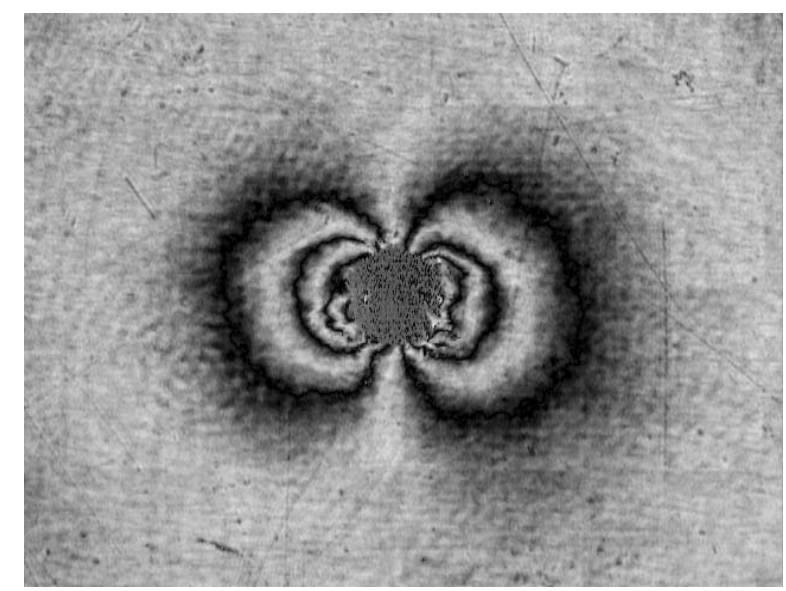

Figure 5.5 (a) In-plane $U$ field fringe pattern $(0.833 \mu \mathrm{m} /$ fringe $)$.

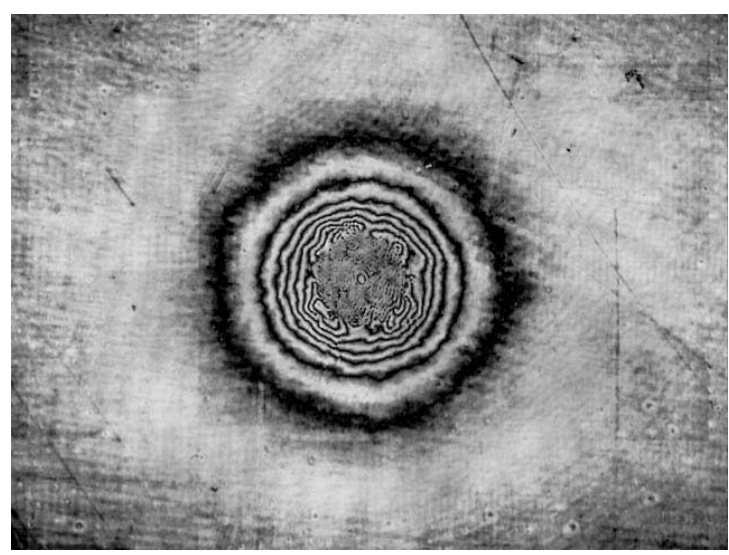

Figure 5.5 (b) Out-of-plane $\mathrm{W}$ field fringe pattern $(0.3164 \mu \mathrm{m} /$ fringe $)$.

The indenter diameter is $1.6 \mathrm{~mm}$. Maximum load is $550 \mathrm{~N}$. Residual $\mathrm{U}$ and $\mathrm{W}$ field is measured by combined Twyman-Green Interferometry and Moiré Interferometry method [58]. Fringe patterns of residual $U$ and $W$ field are shown in Figure 5.5 (a) and 
(b) respectively. The values of $\mathrm{U}$ and $\mathrm{W}$ displacements are obtained by quantification of gray scale value at each point in the image.

Accordingly, finite element simulation is performed based on the model described in Chapter 3. 'Control load' method is employed to reach maximum load 550N. The contact interface between the indenter and specimen is assumed to be frictionless. Then the residual displacement fields ( $\mathrm{U}$ and $\mathrm{W}$ ) are extracted to compare with experimental results, which is shown in Figure 5.6 (a) and (b).

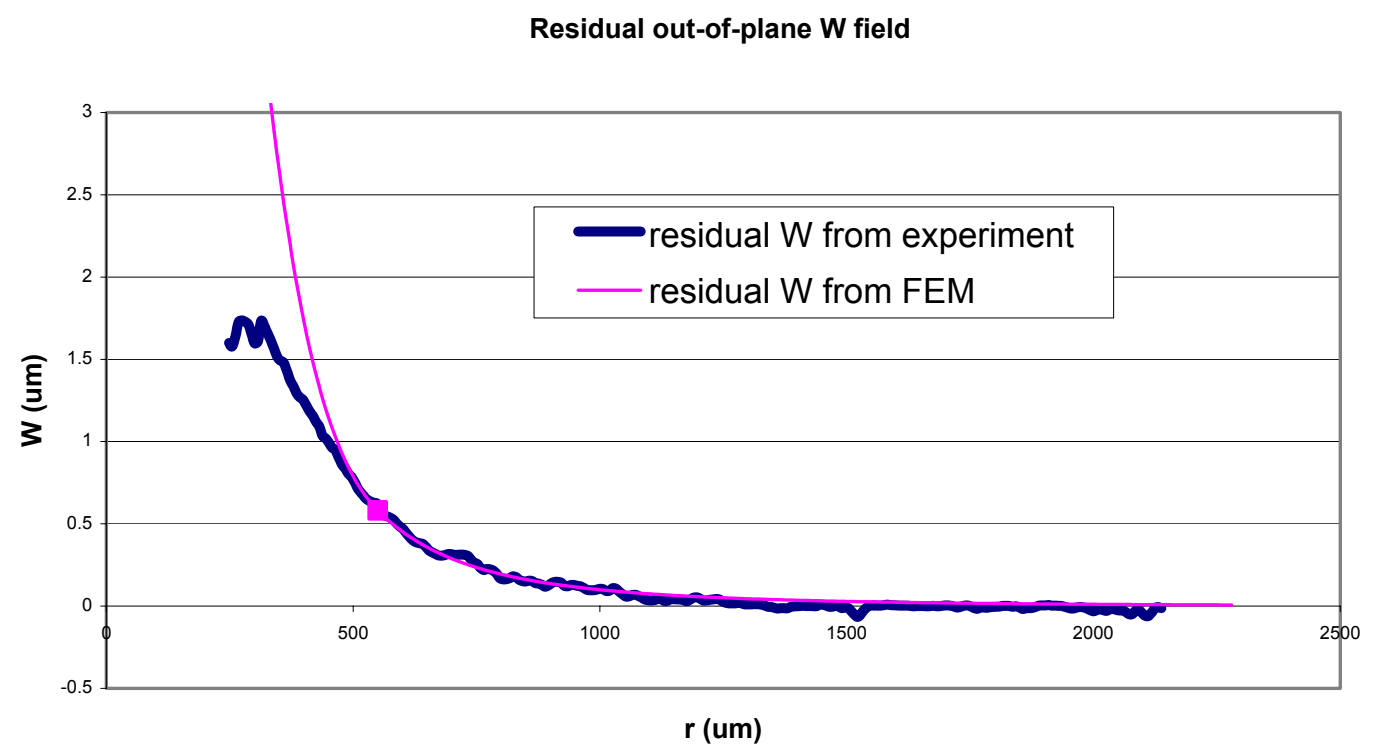

Figure 5.6 (a) Residual out-of-plane W field comparison. 
Residual in-plane U field

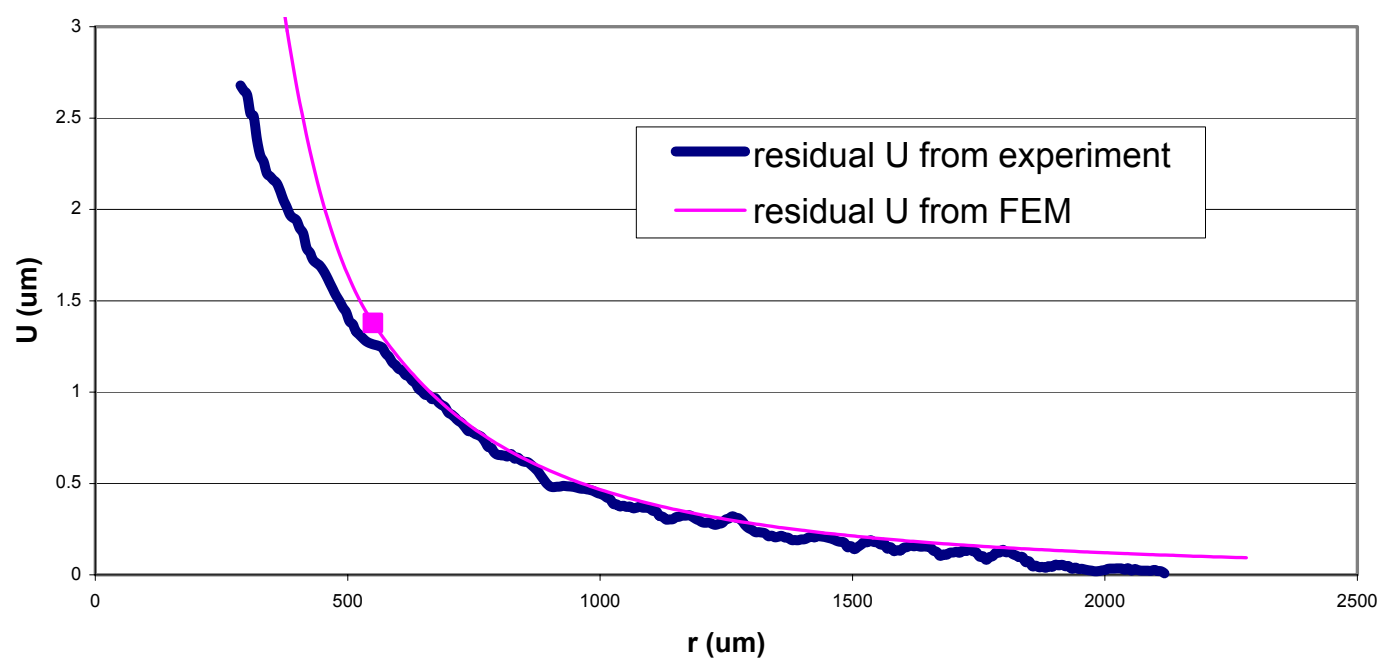

Figure 5.6 (b) Residual in-plane U field comparison.

It is found from the comparison graphs that the finite element results of $\mathrm{U}$ and $\mathrm{W}$ fields are in appreciably good agreement with experimental result in elastic deformation zone. In plastic deformation zone, finite element gives higher value for both $\mathrm{U}$ and $\mathrm{W}$ fields than experiment does. This discrepancy could be attributed to two reasons:

1) Coefficient of friction effect. As discussed in Chapter 4, the displacement fields in the area nearby indenter will be influenced much by the value of coefficient of friction. The inclusion of friction will drastically decrease the $\mathrm{W}$ displacement values, compared with frictionless case.

2) In the vicinity of indenter, the real material would have failed due to large plastic deformation. This failure cannot be simulated in finite element.

The experimental results favorably support the finite element simulations based on current mesh and modeling. Conclusions based on finite element simulation results could be drawn with great confidence. 


\section{Chapter 6}

\section{Data Analysis}

\subsection{Simulation matrix: material library selection}

A large variety of material models are selected to perform the finite element simulation, considering the real engineering materials mechanical properties. Basically three sets of engineering alloys are selected: Aluminum alloys, Steel alloys and lead free solder materials.

Table 6.1 Simulation matrix: material library selection

\begin{tabular}{|l|l|l|l|l|l|l|l|}
\hline \multicolumn{7}{|c|}{ Aluminum alloys (E=69 GPa, $v=0.33)$} \\
\hline $\mathrm{n}=0.09$ & $\mathrm{n}=0.18$ & $\mathrm{n}=0.27$ & $\mathrm{n}=0.36$ & $\mathrm{n}=0.09$ & $\mathrm{n}=0.18$ & $\mathrm{n}=0.27$ & $\mathrm{n}=0.36$ \\
& & & & & & & \\
\hline
\end{tabular}

\begin{tabular}{|l|l|l|l|l|l|l|l|l|}
\hline \multicolumn{2}{|c|}{ Steel (E=200 GPa, v=0.3) } \\
\hline \multicolumn{2}{|c|}{$\sigma_{\mathrm{y}}=242 \mathrm{MPa}$ (mild steel) } & \multicolumn{3}{|c|}{$\sigma_{\mathrm{y}}=500 \mathrm{MPa}$} & \multicolumn{3}{c|}{$\sigma_{\mathrm{y}}=750 \mathrm{MPa}$} \\
\hline $\mathrm{n}=0.1$ & $\mathrm{n}=0.2$ & $\mathrm{n}=0.3$ & $\mathrm{n}=0.1$ & $\mathrm{n}=0.2$ & $\mathrm{n}=0.3$ & $\mathrm{n}=0.1$ & $\mathrm{n}=0.2$ & $\mathrm{n}=0.3$ \\
\hline
\end{tabular}

\begin{tabular}{|c|c|c|c|}
\hline \multicolumn{4}{|c|}{ Lead free solder materials $(E=26.2 \mathrm{GPa}, \mathrm{v}=0.3)$} \\
\hline \multicolumn{4}{|c|}{$\sigma_{\mathrm{y}}=22.5 \mathrm{MPa}(\mathrm{Sn}-3.5 \mathrm{Ag})$} \\
\hline $\mathrm{n}=0.026$ & $\mathrm{n}=0.1$ & $\mathrm{n}=0.2$ & $\mathrm{n}=0.3$ \\
\hline
\end{tabular}

There are altogether 21 materials simulated in the $1.6 \mathrm{~mm}$ diameter spherical indentation test, which include very soft material (lead free solder material) and relatively strong material (steel alloy). Basically, strain-hardening exponent $\mathrm{n}$ values vary for the same $E$ and $\sigma_{y}$ value to evaluate the influence of $n$ value on the indentation data. 
Considering the real material property, for a designated set of materials, Young's modulus doesn't change much (e.g., for aluminum alloys E=69 GPa, for steel alloys $\mathrm{E}=200 \mathrm{GPa}$ ). However, the $\sigma_{\mathrm{y}}$ and $\mathrm{n}$ values lies in a broad range due to different composition and heat treatment in the same set of alloys.

In general, the indenter is impressed $1 / 5$ of its diameter. In this case, since the indenter diameter is $1.6 \mathrm{~mm}$, the maximum depth of $320 \mu \mathrm{m}$ is simulated for all the material models. The applied force at each increment is then calculated by summation of bottom line reaction force of the half space. The incremental increase of the depth to its peak value followed by the incremental unloading until the contact pair separates will be referred to as a "load cycle".

\subsection{Tabor's stress strain relation}

In Tabor's empirical work in 1950's [39], it was pointed out that for spherical indentation, the Meyer's hardness, which was defined as the mean contact pressure $p_{m}=\frac{P}{A_{p r o j}}$, has a close agreement with the uniaxial stress-strain curve. Given the definition of indentation stress and strain:

$$
\left\{\begin{array}{c}
\varepsilon_{i}=0.2 \frac{d}{D} \\
\sigma_{i}=\frac{p_{m}}{2.8}
\end{array}\right.
$$

where $\mathrm{d}$ is the indentation impression diameter, $\mathrm{D}$ is the ball indenter diameter, Tabor claimed that this indentation stress-strain relation is equal to the uniaxial compression stress-strain curve in the fully plastic region. The conclusion was based on experiments on two materials: mild steel and anneal copper. It was also pointed out that this relationship is independent of indenter size. 
Here, for each material model, both the contact diameter $\mathrm{d}$ and load $\mathrm{P}$ could be extracted from the FEM results for each loading increment. Once plugged into Equation (6.1), the indentation stress and strain under different loading status are plotted as discrete data points (Fig. 6.2.1). Then, the results are compared with the input material stress strain curve, as shown in Figs 6.2.1 (a)-(f) for 21 material models.

Tabor's stress strain relation Material model set: $E=69 \mathrm{GPa}$, sy=275 MPa

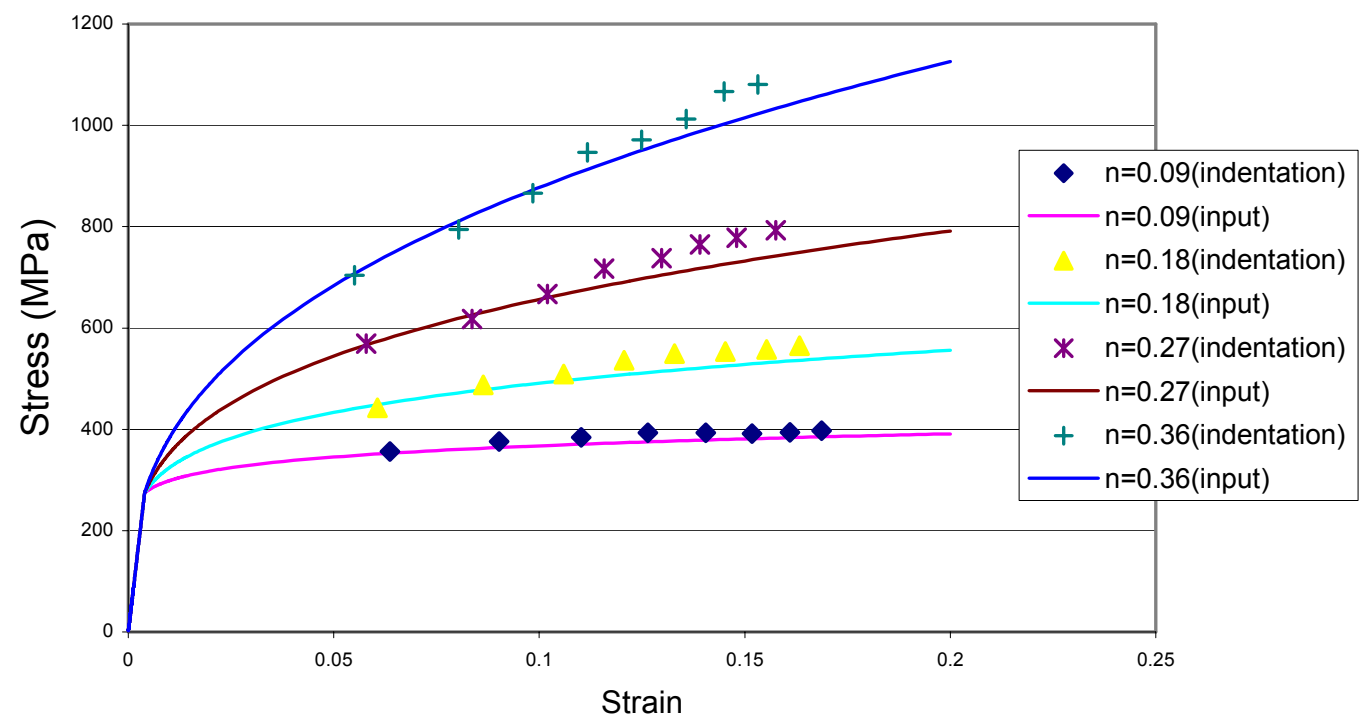

Figure 6.2.1 (a) Tabor's stress strain relation for material set E=69 GPa, $\sigma_{Y}=275 \mathrm{MPa}$, $\mathrm{n}=0.09,0.18,0.27,0.36$ respectively. 
Tabor's stress strain relation

Material model set: E=69 GPa, sy=500 MPa

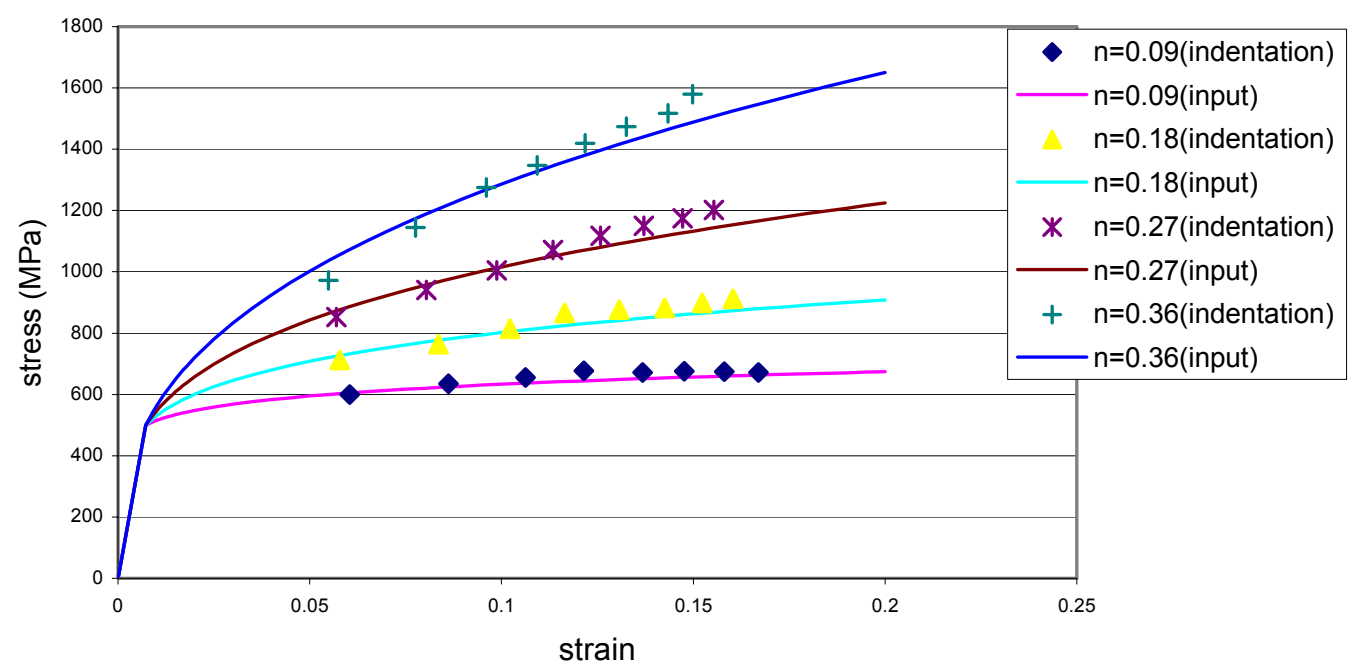

Figure 6.2.1 (b) Tabor's stress strain relation for material set E=69 GPa, $\sigma_{Y}=500 \mathrm{MPa}$, $\mathrm{n}=0.09,0.18,0.27,0.36$ respectively.

Tabor's stress strain relation

Material model set: $\mathrm{E}=200 \mathrm{GPa}$, sy=242 $\mathrm{MPa}$

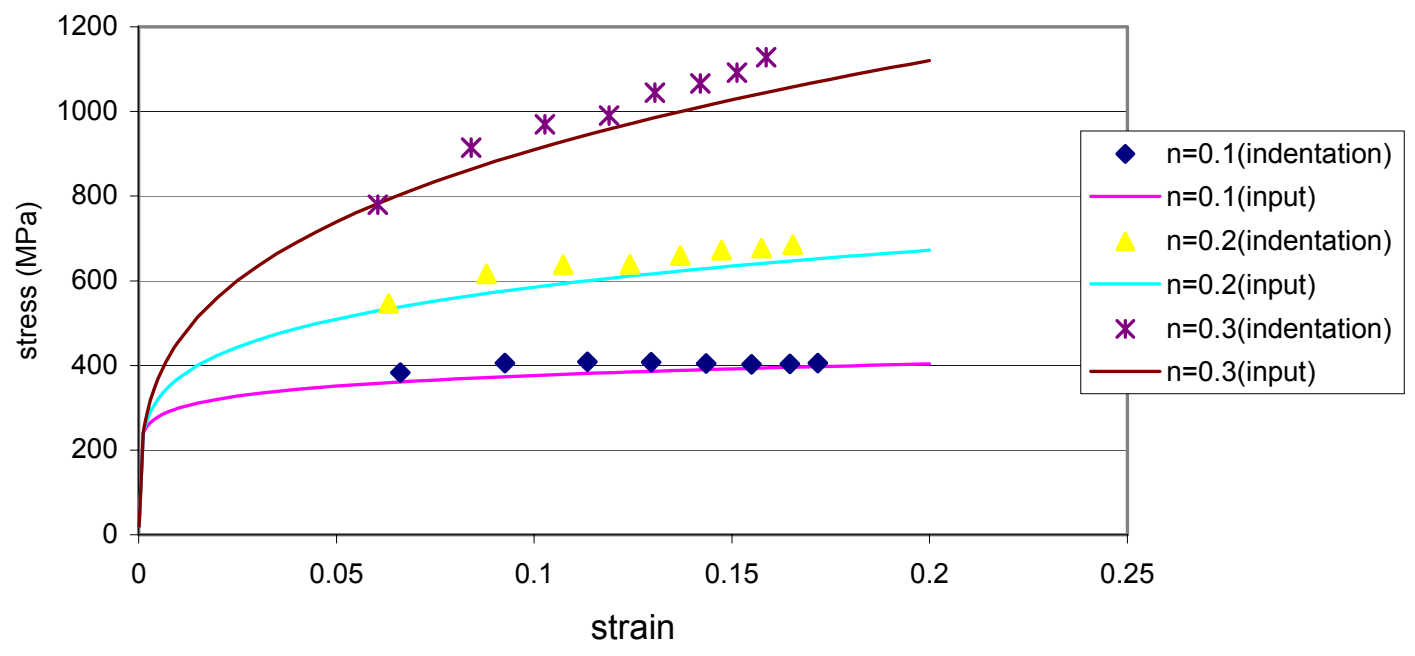

Figure 6.2.1 (c) Tabor's stress strain relation for material set E=200 GPa, $\sigma_{Y}=242 \mathrm{MPa}$, $\mathrm{n}=0.1,0.2,0.3$ respectively. 
Tabor's stress strain relation

Material model set: $\mathrm{E}=200 \mathrm{GPa}, \mathrm{sy}=500 \mathrm{MPa}$

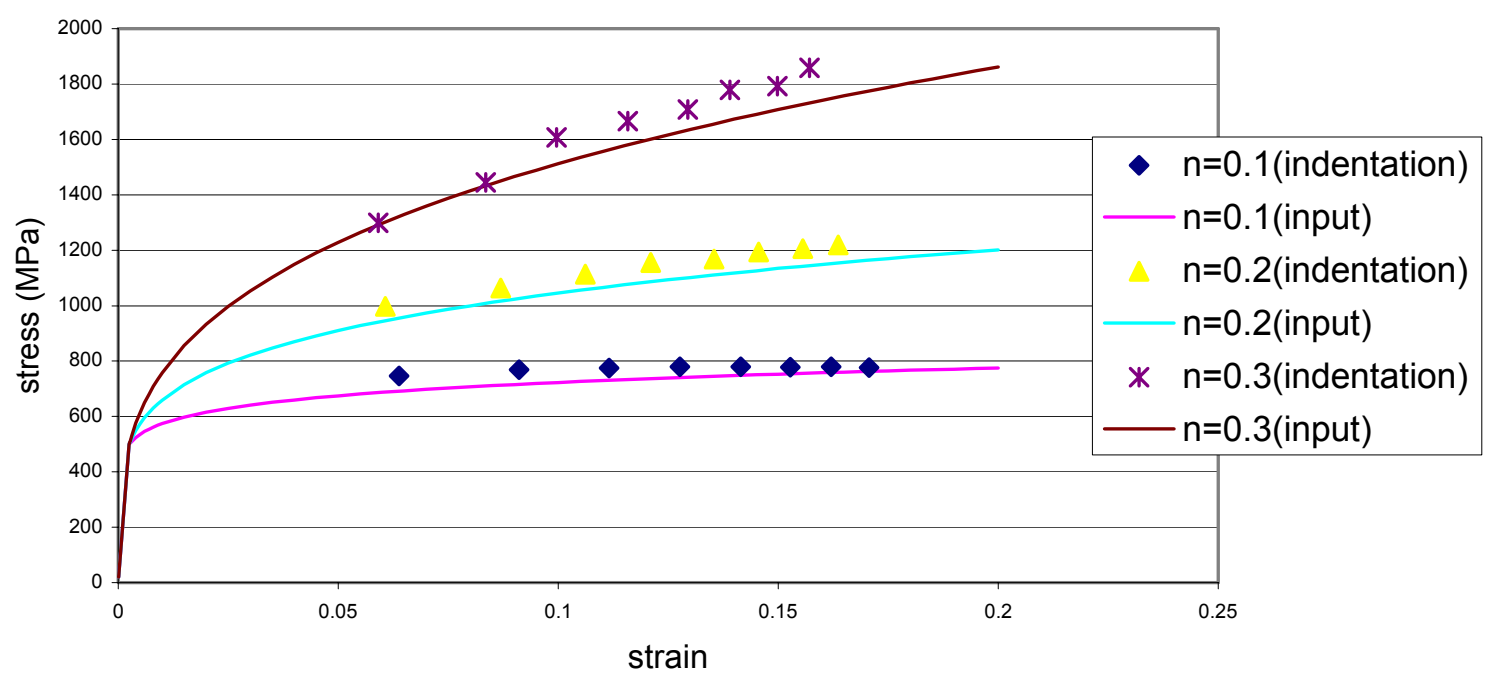

Figure 6.2.1 (d) Tabor's stress strain relation for material set E=200 GPa, $\sigma_{Y}=500 \mathrm{MPa}$, $\mathrm{n}=0.1,0.2,0.3$ respectively.

Tabor's stress strain relation Material model set: E=200 GPa,sy=750 MPa

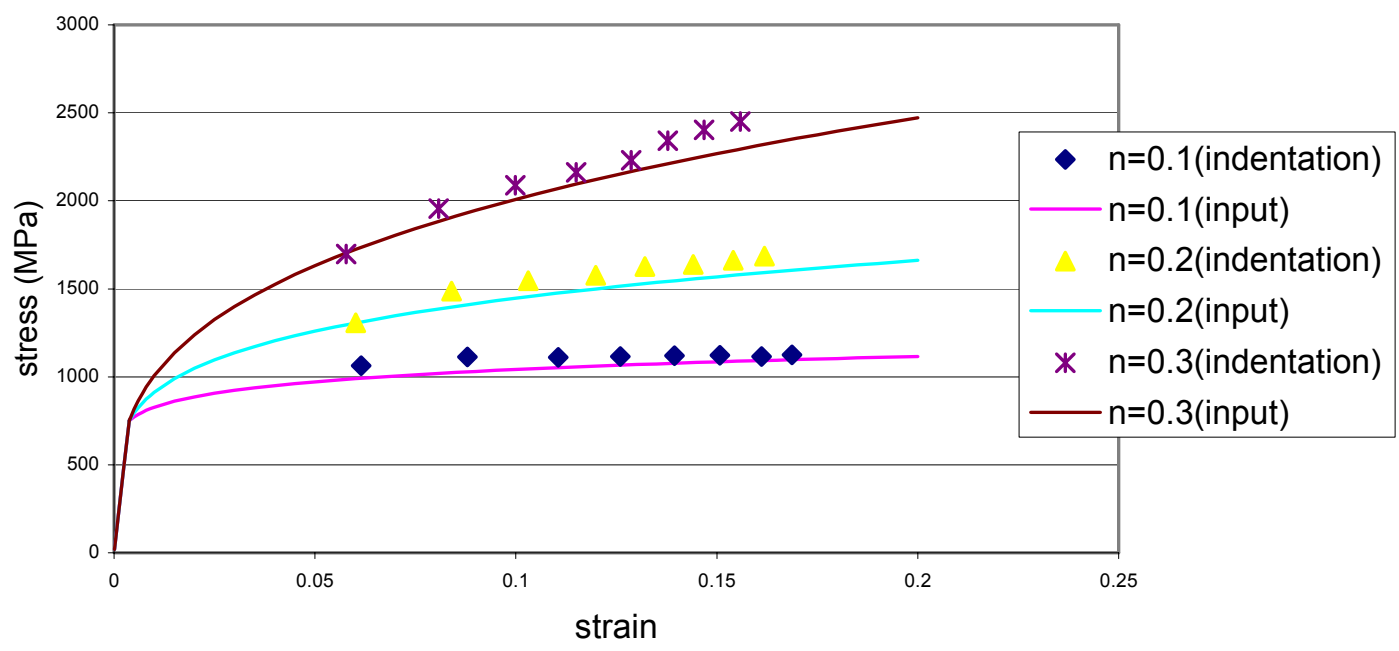

Figure 6.2.1 (e) Tabor's stress strain relation for material set E=200 $\mathrm{GPa}, \sigma_{\mathrm{Y}}=750 \mathrm{MPa}$, $\mathrm{n}=0.1,0.2,0.3$ respectively. 
Tabor's stress strain relation

Material model set: $\mathrm{E}=26.2 \mathrm{GPa}, \mathrm{sy}=22.5 \mathrm{MPa}$

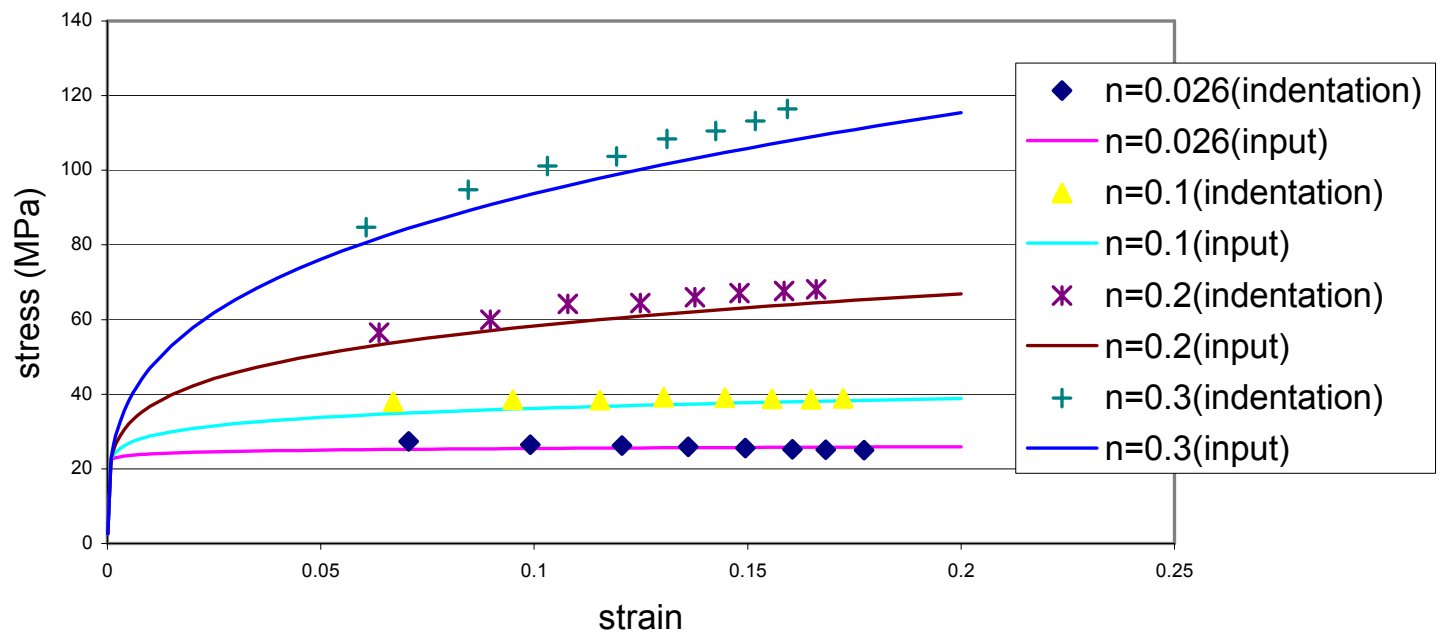

Figure 6.2.1 (f) Tabor's stress strain relation for material set $\mathrm{E}=26.2 \mathrm{GPa}, \sigma_{\mathrm{Y}}=22.5 \mathrm{MPa}$, $\mathrm{n}=0.1,0.2,0.3$ respectively.

Results show good agreement in the fully plastic region (deep indentation) between the Tabor's indentation stress strain and the actual stress strain curve (input to the FE model) for all the 21 materials. The error percentage from all data points is limited within $8 \%$. When the indentation diameter is relatively small $(d / D<0.1)$, the material deformation is affected largely by the elastic property of the material and Tabor's relationship is not consistent with the actual stress strain curve. That is why only large strain data are comparable. Previous literature described this as 'fully plastic condition'.

The progress of indentation is divided into three stages according to the response of indented material: elastic, elastic-plastic, and fully plastic $[3,55]$. This conclusion is verified by current FE simulations. 


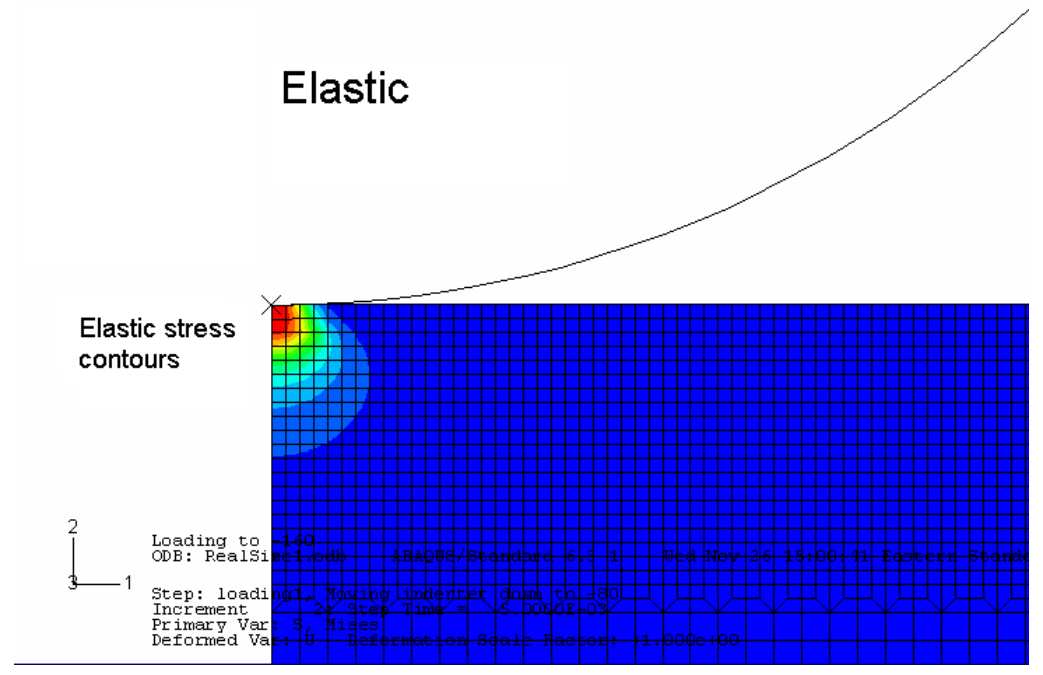

Figure 6.2.2 Finite element graph of plastic zone expansion during ball indentation (a) Elastic regime

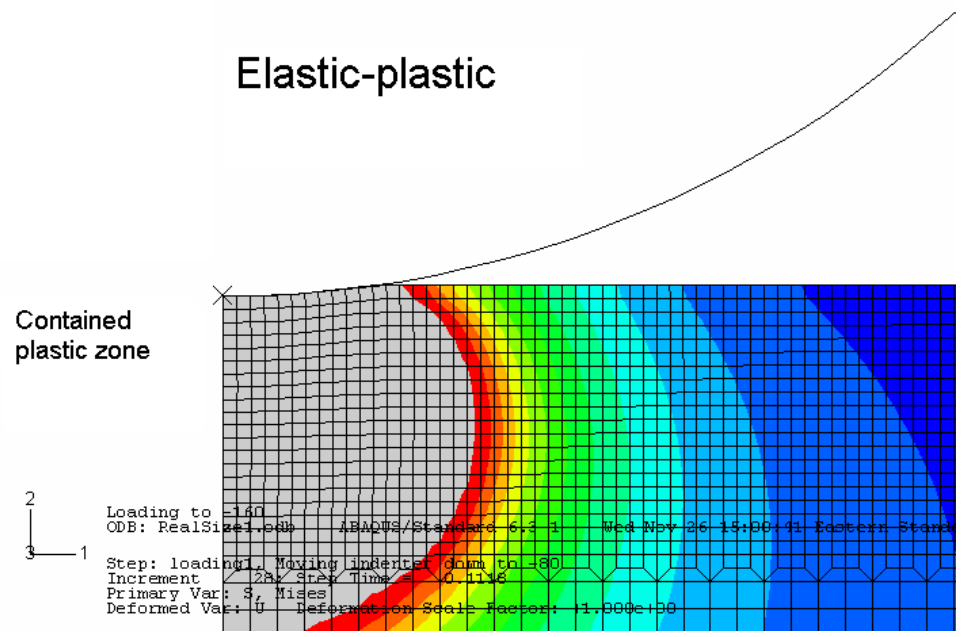

Figure 6.2.2 (b) Elastic-plastic regime 


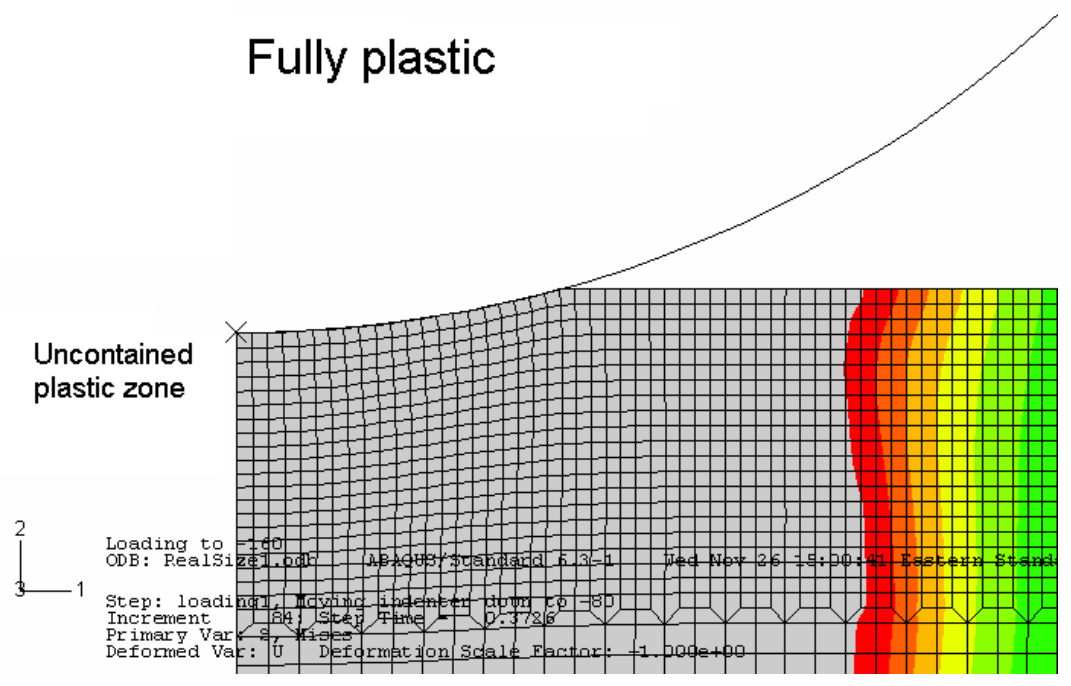

Figure 6.2.2 (c) Fully plastic regime

It is pointed out in Tabor's book [39] that the Tabor's relation Equation (6.1) is only applicable under 'fully plastic' condition, which indicates relatively deep indentation. In Tabor's work, it is said that fully plasticity will be reached for mild steel when $\mathrm{d} / \mathrm{D}$ is greater than about 0.1 and for copper at a smaller value.

The boundary between the elastic-plastic regime and the fully plastic regime is determined by a nondimensional variable $E / \sigma_{y} \cdot \tan \gamma$ and its value is about 30 [3], in which $\gamma$ is contact angle and it is calculated by $\sin \gamma=a / R=d / D$. It is derived from the above analysis that the minimum strain value that could be obtained from Tabor's relation, is:

$$
\varepsilon_{\min }=0.2 \sin \left(\tan ^{-1} 30 \varepsilon_{e}\right)
$$

where $\varepsilon_{e}=\sigma_{y} / E$, which is the elastic strain when yielding.

Direct observation finds out that for large $n$ value $(n>0.2)$, the Tabor's relation usually gives a higher value of stress especially for large plastic strain part. Each 
material model in Figure6.2.1 (a) to (f) is plotted out in Figure 6.2.3. Average of $p_{m} / \sigma$ of all data points is taken.

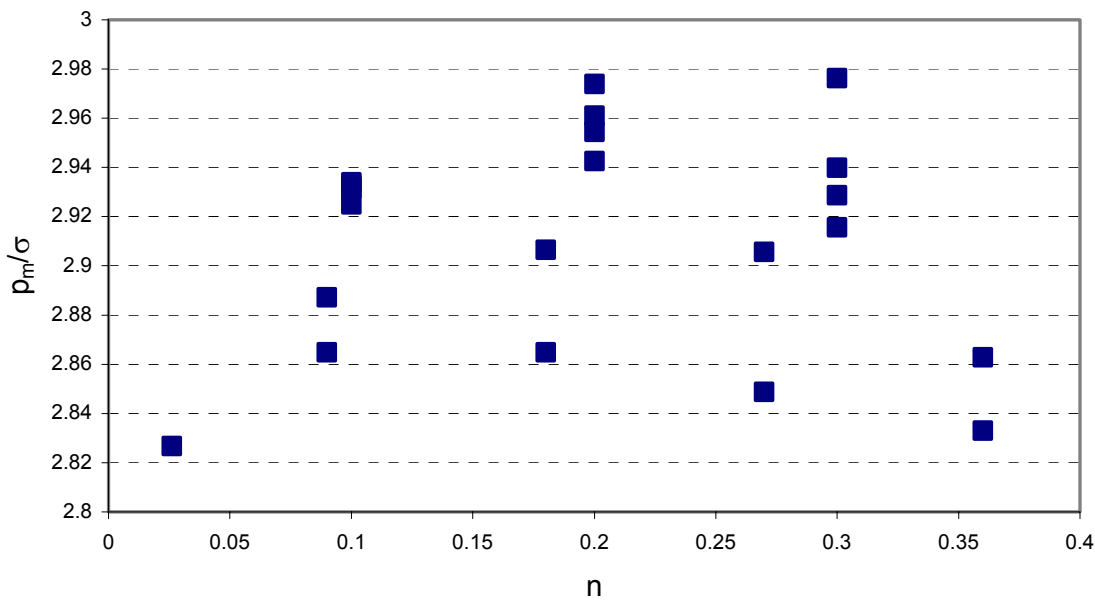

Figure6.2.3 $p_{m} / \sigma$ fluctuation

It is found that the value of $p_{m} / \sigma$ fluctuates between 2.82 to 2.98 . The fluctuation range is small and Tabor's relation is good in predicting post yielding stress strain behavior for different materials. From this work, it is proposed that the indentation stress could be modified as: $\sigma_{i}=p_{m} / 2.9$

Another interesting discovery of this research is that the indentation stress $\sigma_{i}$ approximately equals the von Mises effective stress at the contact separation circle on the surface.

Tabor's relationship provides a simple, reliable experimental method to obtain the material post-yielding stress strain curve from the indentation test. The advantage of this method is that it is not necessary to assume a mathematical model (power law, bilinear, etc.) to describe the uniaxial tensile stress strain curve. The discrete data points are obtained directly from the indentation. 


\subsection{Load depth curve}

Load depth (P-hs) curve is the straightforward characterization of indentation results, which could be precisely obtained by instrumented indentation equipment. For each material model, finite element simulation results gave a set of $\mathrm{P}$ and hs data for each loading step and then these data points are plotted in Excel.

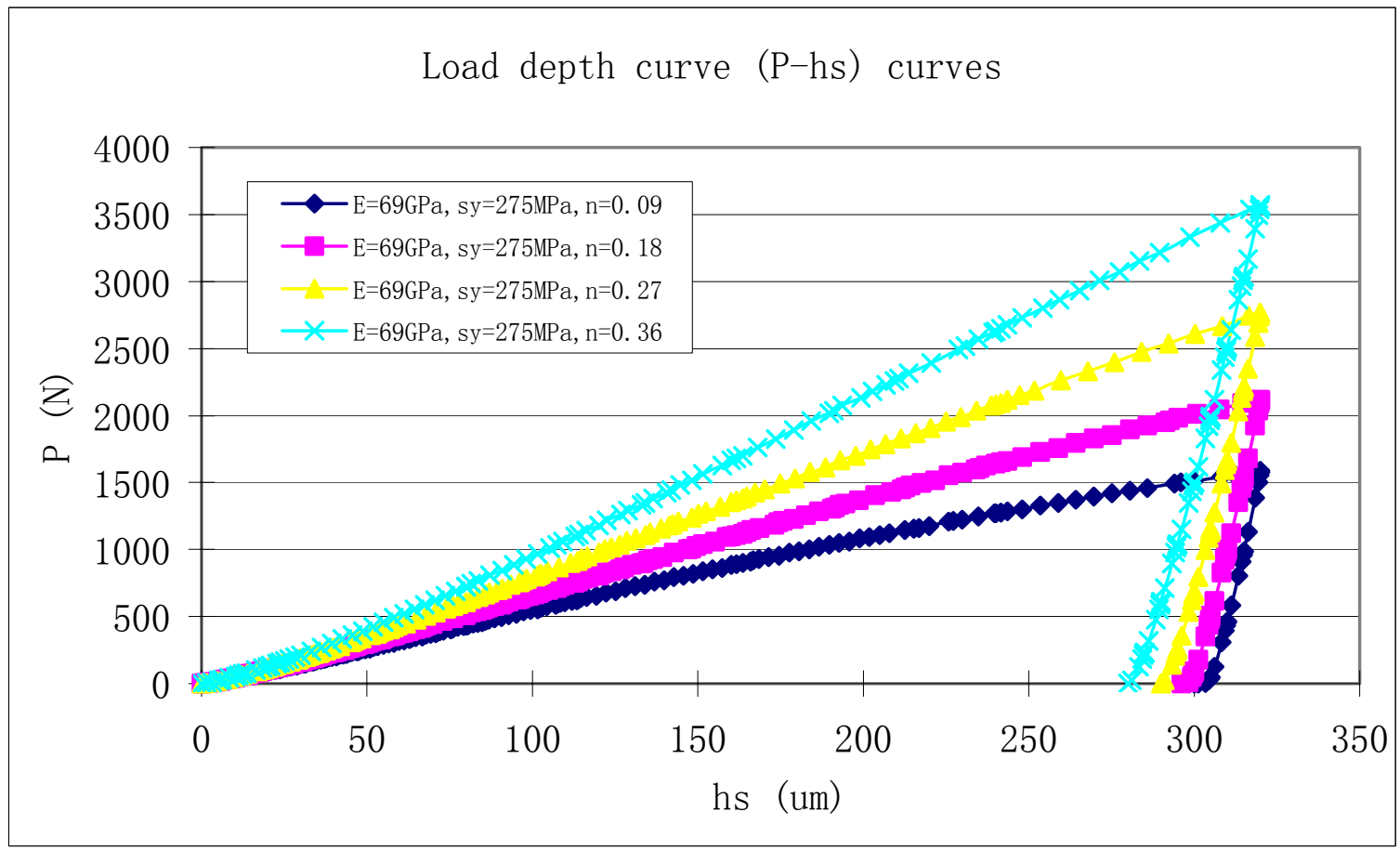

Figure 6.3.1 (a) Load depth curves for material set $\mathrm{E}=69 \mathrm{GPa}, \sigma_{\mathrm{y}}=275 \mathrm{MPa}, \mathrm{n}=0.09$, $0.18,0.27,0.36$ respectively. 


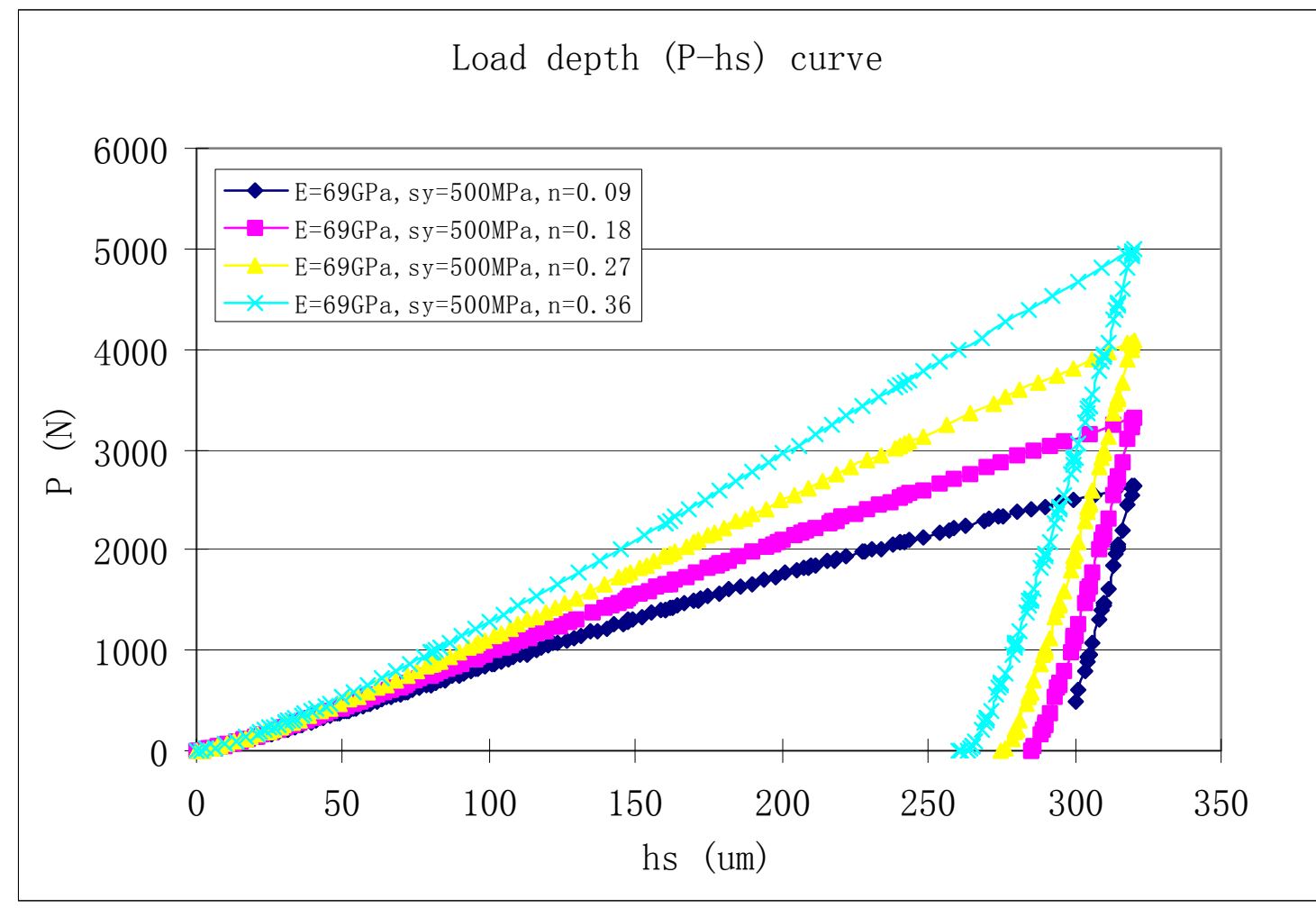

Figure 6.3.1 (b) Load depth curves for material set E=69 GPa, $\sigma_{y}=500 \mathrm{MPa}, \mathrm{n}=0.09$, $0.18,0.27,0.36$ respectively.

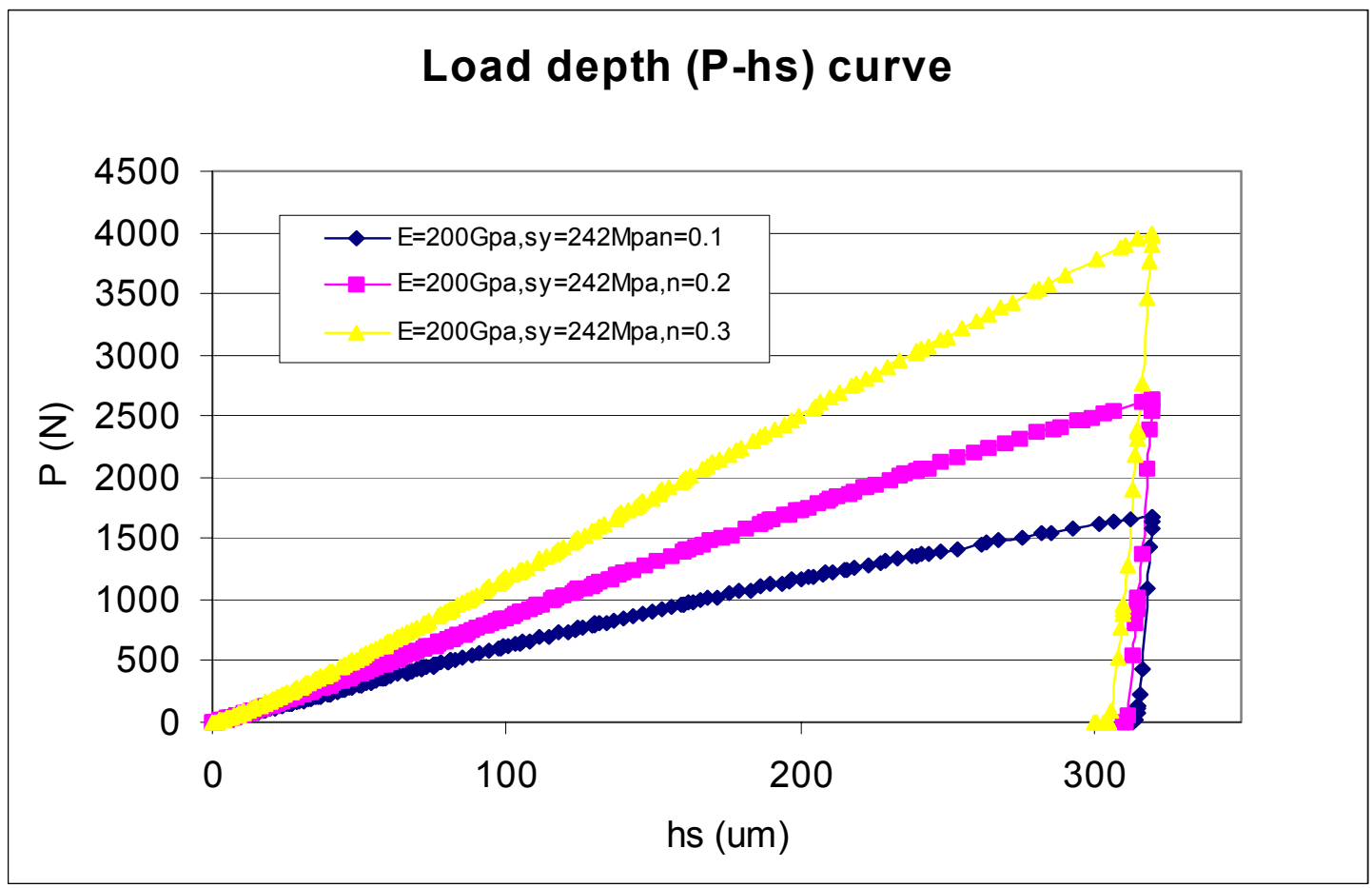

Figure 6.3.1 (c) Load depth curves for material set $\mathrm{E}=200 \mathrm{GPa}, \sigma_{\mathrm{y}}=242 \mathrm{MPa}, \mathrm{n}=0.1,0.2$, 0.3 respectively. 


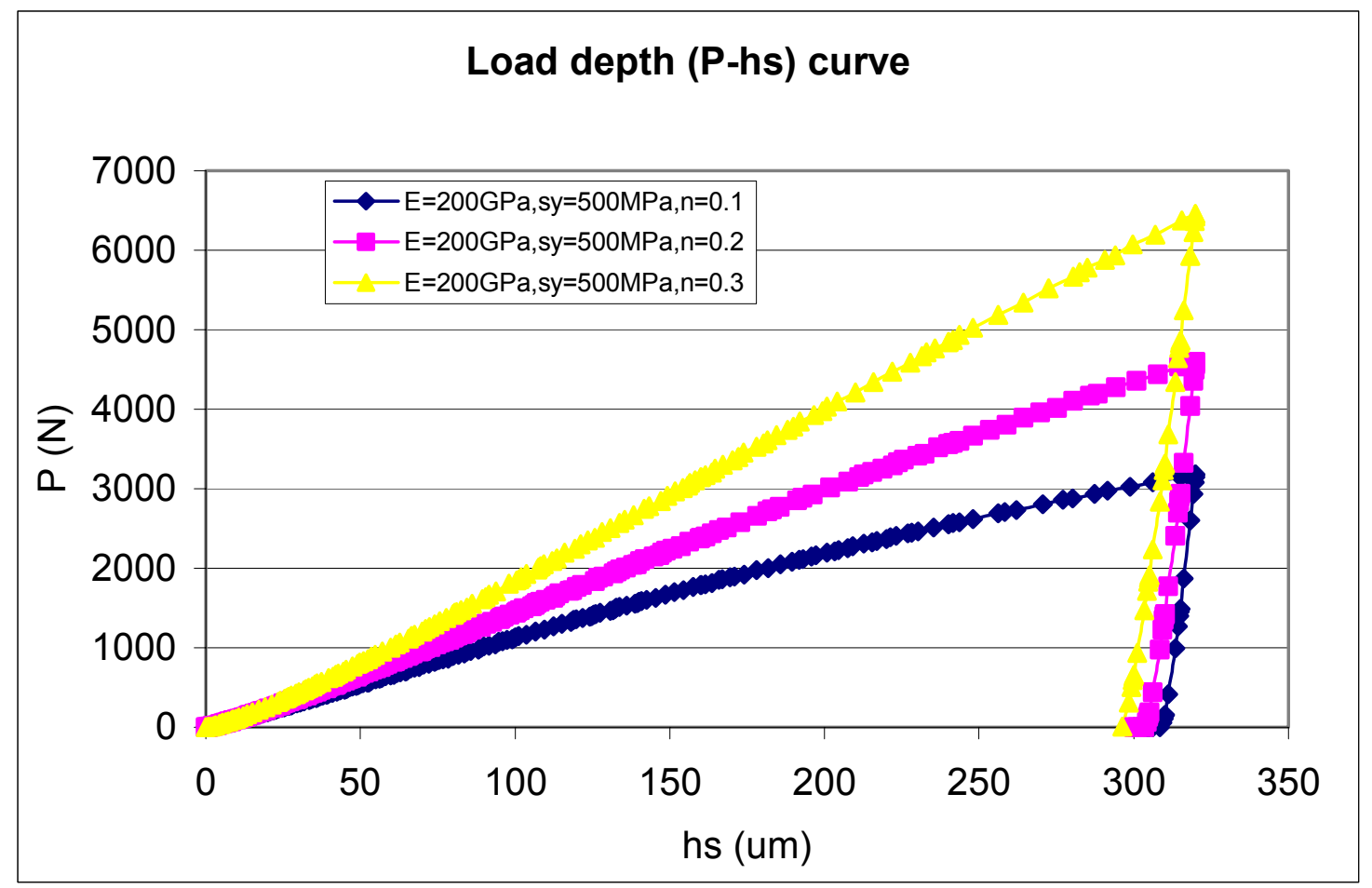

Figure 6.3.1 (d) Load depth curves for material set E=200 GPa, $\sigma_{\mathrm{y}}=500 \mathrm{MPa}, \mathrm{n}=0.1,0.2$, 0.3 respectively.

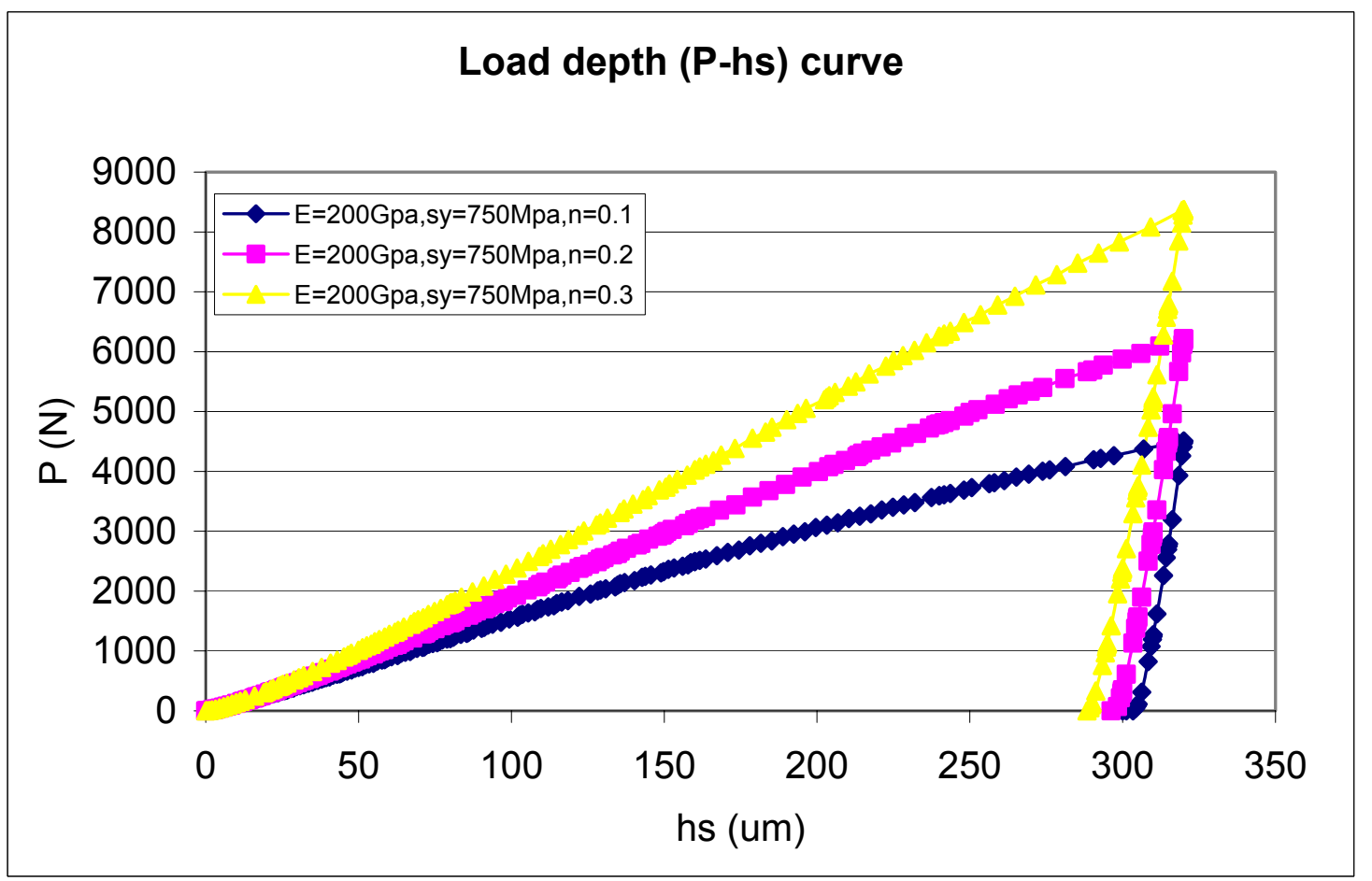

Figure 6.3.1 (e) Load depth curves for material set $\mathrm{E}=200 \mathrm{GPa}, \sigma_{\mathrm{y}}=750 \mathrm{MPa}, \mathrm{n}=0.1,0.2$, 0.3 respectively. 


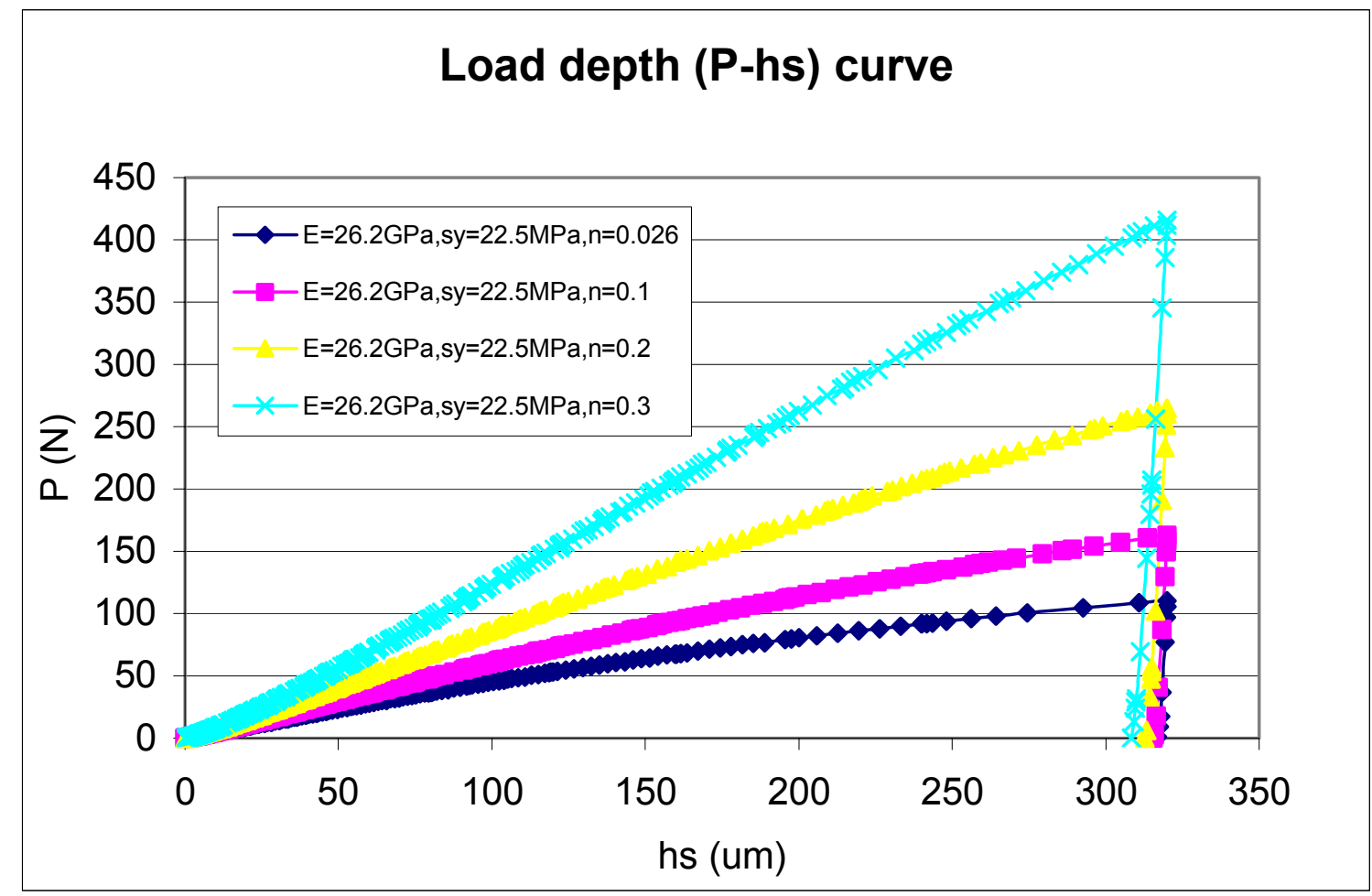

Figure 6.3.1 (f) Load depth curves for material set $\mathrm{E}=26.2 \mathrm{GPa}, \sigma_{\mathrm{y}}=22.5 \mathrm{MPa}, \mathrm{n}=0.026$, $0.1,0.2,0.3$ respectively.

The relationship between P-hs curve and material properties is directly observed.

It is found that with the increase of Young's modulus, yield stress or hardening exponent, the load value (P) gets higher for given depth (hs), which implies for harder and stronger material, a larger load is needed to reach same indentation depth. This conclusion complies with common sense.

In addition to providing some basic information of the indented material, the slope of initial unloading part of the P-hs curve, which is defined as indentation stiffness, is used to quantify material elastic property. The unloading curves shown in Figure 6.3.1 exhibit an initially linear response. Assuming the initial unloading of the indentation is pure elastic rebound, material Young's modulus can be derived from Equation (2.13). 
One of the most delicate problems in the instrumented indentation equipment is how to determine the real contact radius a (and projected contact area $A=\pi a^{2}$ ), which was discussed in detail in Chapter 2. This problem does not exist in this work since the finite element results could provide accurate value of a as long as the mesh underneath the indenter is dense enough.

Table 6.2 Using indentation stiffness to calculate Young's modulus.

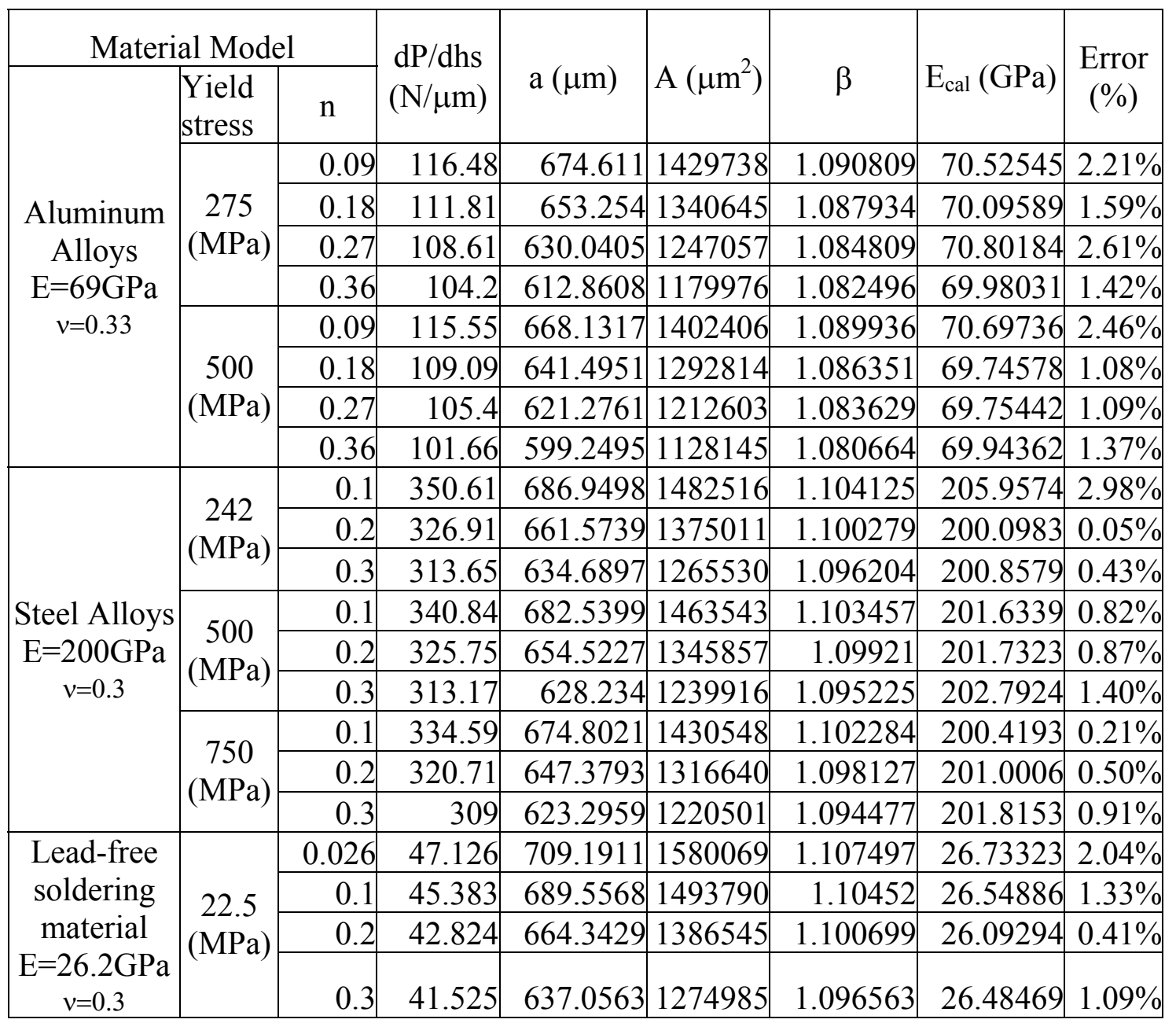

In this work, Equation (2.13) is employed to calculate Young's modulus, in which the correction factor $\beta$ is calculated by Equation (2.16) for spherical indenter. Contact 
radius is given directly from FEM results and projected contact area at maximum load could be calculated.

Comparisons in Table 6.2 indicate good agreement of the input and calculated Young's modulus from FEM simulation results. The error percentage is less than 3\%. The results show the method to calculate material Young's modulus through indentation stiffness is reliable and accurate.

As the unloading progresses, the curves in Figure 6.3.1 show a nonlinearity, which becomes more pronounced with increasing strain hardening. Bhattacharya and Nix [10] have shown that materials with a larger yield strength-to-elastic modulus ratio (more elastic strain percentage in total strain) exhibit greater curvature in the nonlinear portion of the unloading curve, which is in agreement with the results presented in Figures 6.3.1

Another observation of the load-depth curve is the amount of elastic rebound depth, which is defined as:

$$
E R D=h_{s}^{\max }-h_{s}^{r}
$$

where:

$h_{s}^{\max }$ is the indentation depth at maximum load, and

$h_{s}^{r}$ is the indentation depth when load is totally removed (residual).

It is found that variation of all the three parameters in material models would cause the change of ERD value. Specifically the ERD value would increase with the increase of $\sigma_{y}$ and n, while it decreases with the increase of Young's modulus, E. 


\subsection{Surface displacement and strain fields}

\subsubsection{Surface displacement fields}

The spherical indentation is an axisymmetric problem so the loop displacement $u_{\theta}=0$ in cylindrical coordinate system. The non-zero displacement components on surface are in-plane radial displacement $u_{r}$ (or $\mathrm{U}$ ) and out-of-plane vertical displacement $u_{z}($ or $\mathrm{W})$.

\subsubsection{Single node tracing for loading process}

The variation of $\mathrm{W}$ value of two surface nodes vs. indentation depth is plotted.

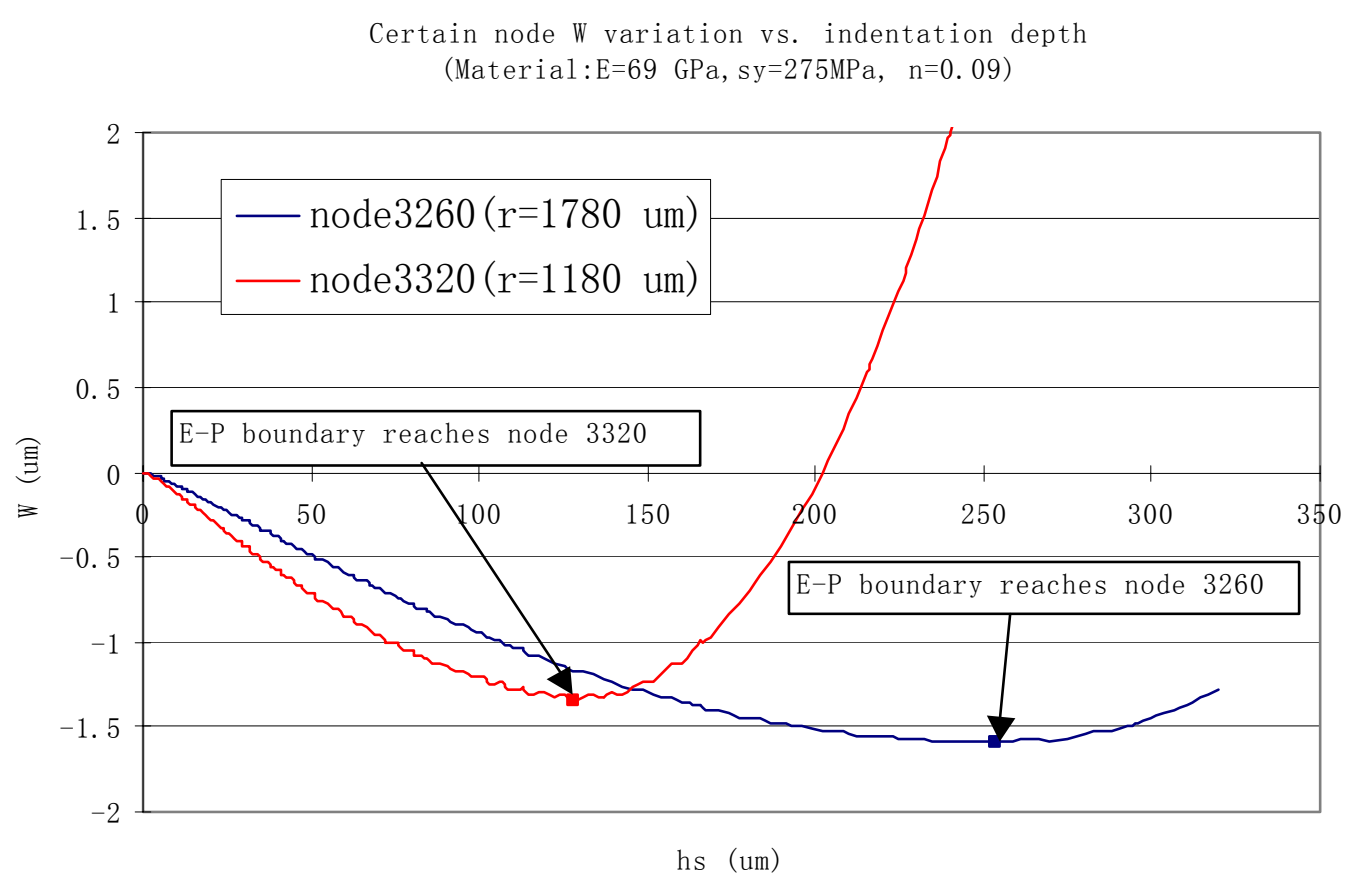

Figure 6.4.1 Two nodes $\mathrm{W}$ displacement variation vs. indentation depth.

From the above plot, it is observed the point on surface would sink first due to the compression of the indenter. With the increasing of indentation depth, surface piling-up will expand. When the expansion reaches the point we are tracing, the $\mathrm{W}$ displacement will start to go up. 
Further observation found that the transition point from $\mathrm{W}$ decreasing to increasing corresponds to the loading status at which elastic-plastic boundary reaches this node. So potentially from the in-situ measurement of $\mathrm{W}$ field, the elastic-plastic boundary could be determined for certain loading status.

One thing needed to point out is this method is only applicable for piling-up material. For sinking-in material, the relationship between the $\mathrm{W}$ variation trend and elastic-plastic boundary does not exist.

\subsubsection{Surface displacement fields at certain loading status}

Then surface displacement fields ( $U$ and $W$ ) contours are plotted for both maximum loading status and residual (totally unloading) status.

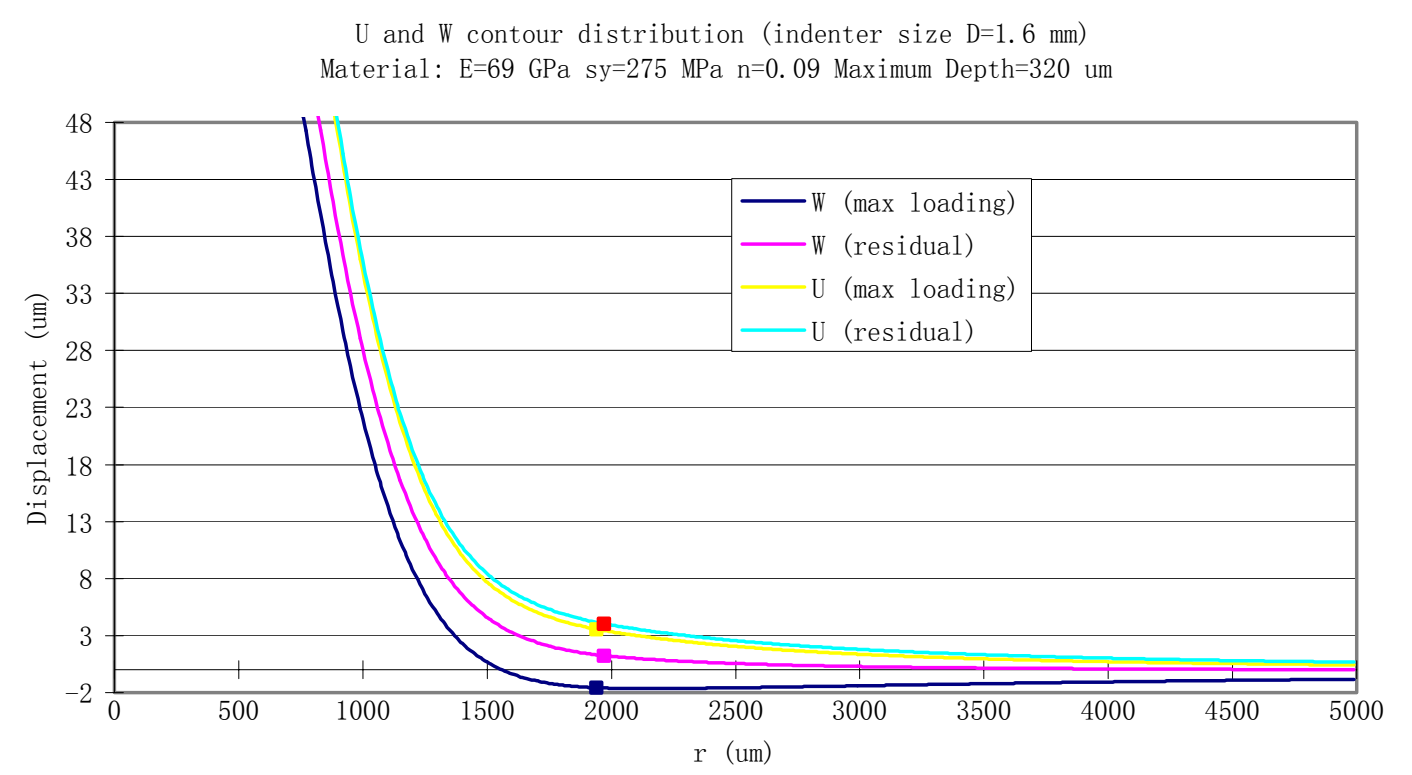

Figure 6.4.2 (a) $\mathrm{U}$ and $\mathrm{W}$ contour distribution at maximum loading and total unloading status $(\mathrm{n}=0.09)$. 


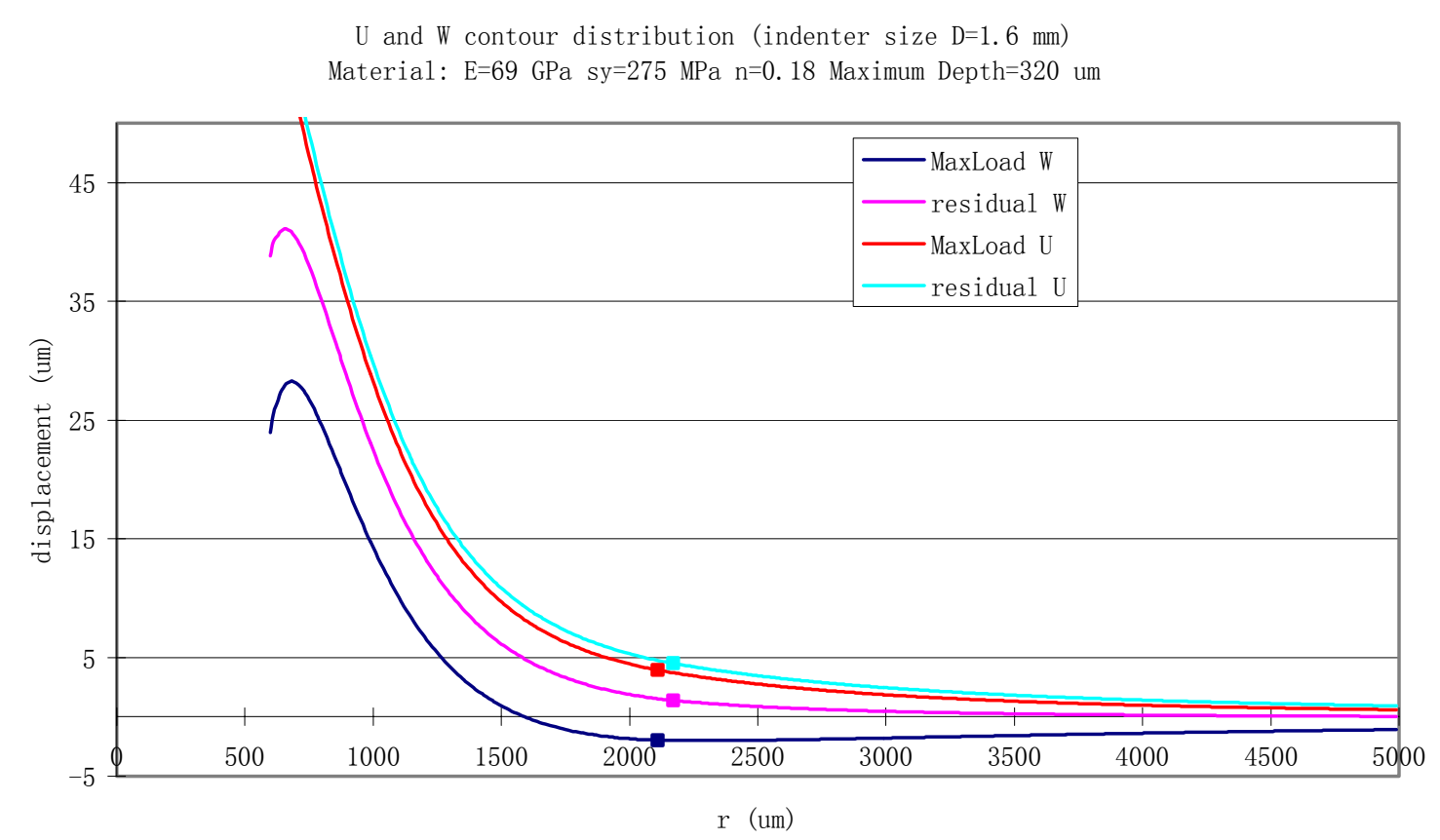

Figure 6.4.2 (b) $\mathrm{U}$ and $\mathrm{W}$ contour distribution at maximum loading and total unloading status $(\mathrm{n}=0.18)$.

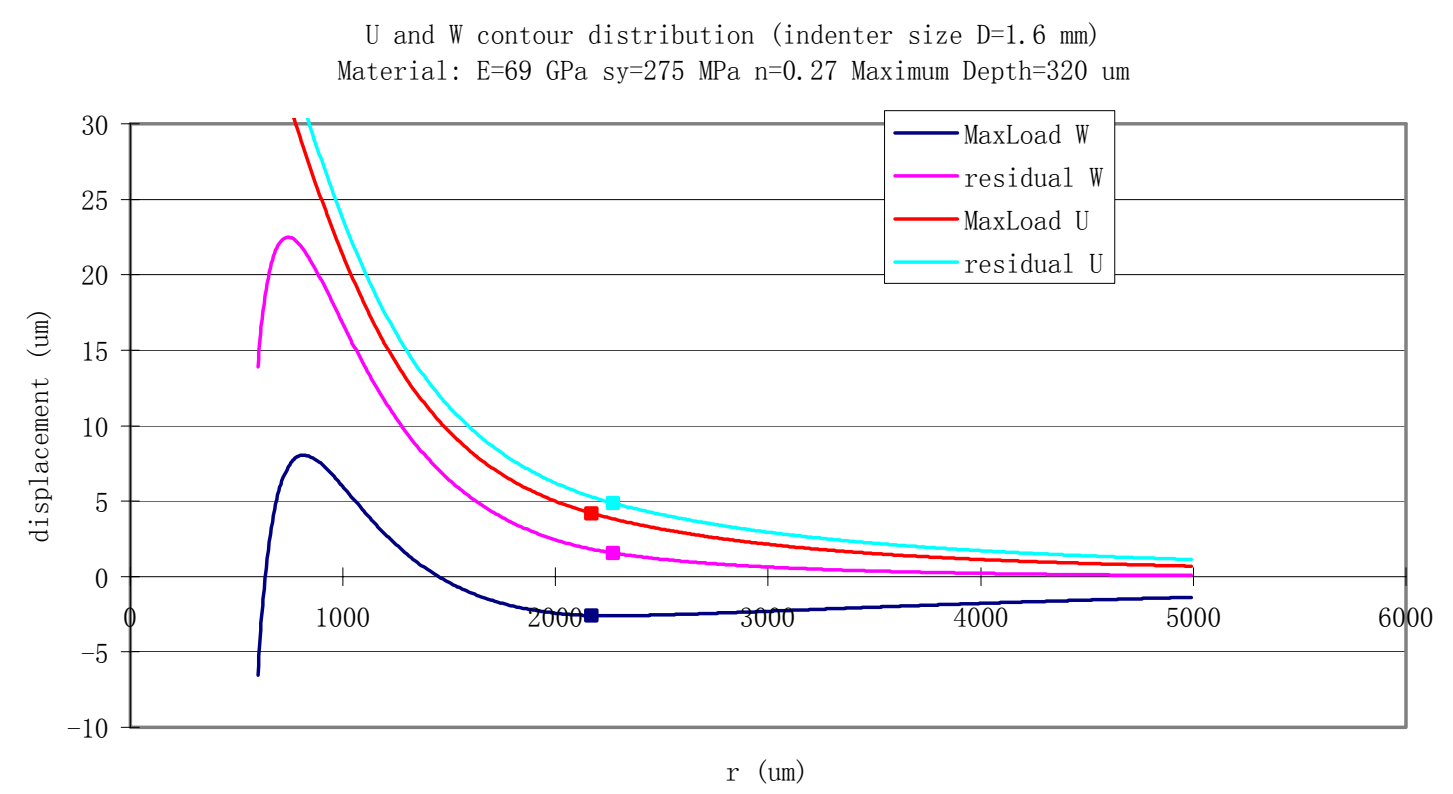

Figure 6.4.2 (c) $\mathrm{U}$ and $\mathrm{W}$ contour distribution at maximum loading and total unloading status $(\mathrm{n}=0.27)$. 


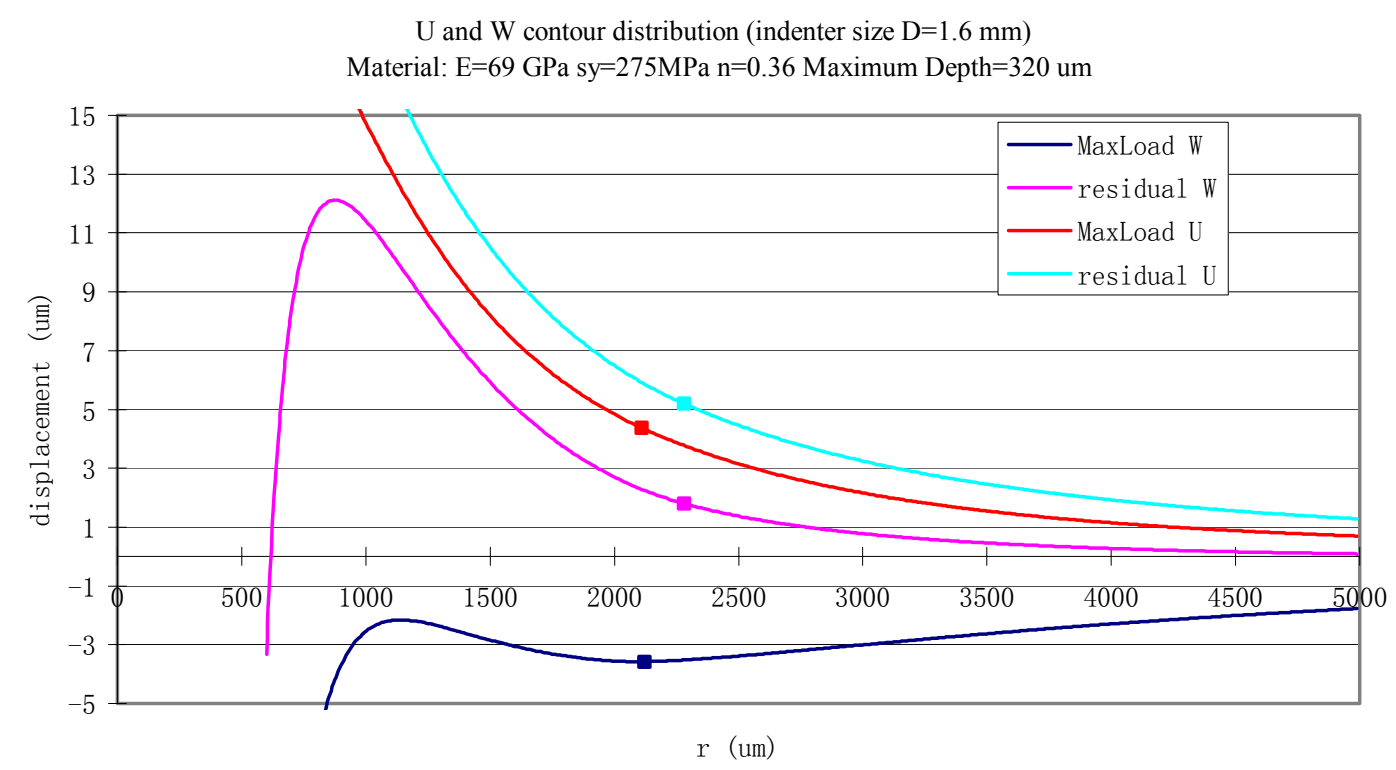

Figure 6.4.2 (d) $\mathrm{U}$ and $\mathrm{W}$ contour distribution at maximum loading and total unloading status $(\mathrm{n}=0.36)$.

Several conclusions could be drawn from above plots:

1. The amount of piling-up and sinking-in is correlated with the strain-hardening exponent of the materials. It is found that sinking-in predominates in materials where $\mathrm{n}>0.2$, and piling-up predominates while $\mathrm{n}<0.2$, which is consistent with the conclusion from J. Alcala [18]. Also the variation trend is consistent with the Hertz elastic solution, in which for pure elastic material $(n=1)$, surface deformation is sinking-in and $\mathrm{h} / \mathrm{hs}=0.5$.

2. U field is always positive, which means the material is pushed out in the radial direction during indentation. Instead of retracting back, the material is moving further away from the indenter during unloading procedure.

3. The residual status of piling up or sinking in is strongly affected by unloading from the peak indentation load. W field is always getting a positive increment during unloading procedure, no matter for piling up or sinking in materials. 
Hence, even for sinking in materials, the residual $\mathrm{W}$ field could indicate piling up surface profile, as shown in Figure 6.4.2 (d).

4. W field changes much more than $U$ field, comparing the maximum loading status and residual status. This is especially obvious for small work hardening materials. Since the unloading procedure is assumed to be pure elastic rebound, this could be explained by Hertz analytical solution. For U field,

$$
u_{r}=-\frac{(1-2 v)(1+v)}{3 E} \frac{a^{2}}{r} \frac{3}{2} p_{m}\left[1-\left(1-\frac{r^{2}}{a^{2}}\right)^{3 / 2}\right]
$$

Such calculations indicate that $\mathrm{U}$ field changing is small. The details of $\mathrm{W}$ field variation during unloading are to be discussed in Section 6.6.

5. The elastic-plastic boundary is corresponding to the characteristic point on the surface contour, which is close to the lowest point in the maximum loading $\mathrm{W}$ distribution plot, no matter for piling-up or sinking-in materials.

6. The elastic-plastic boundary will expand a little bit during the procedure of unloading, which indicates the residual plastic zone is a little larger than the maximum loading plastic zone.

\subsubsection{Surface strain fields}

\subsubsection{Strain components}

The surface strain fields could be obtained from displacement fields through kinematic relationship. The kinematic equations are listed below for axisymmetric problems: 


$$
\left\{\begin{array}{c}
\varepsilon_{r}=\frac{\partial u_{r}}{\partial r} \\
\varepsilon_{\theta}=\frac{u_{r}}{r} \\
\varepsilon_{z}=\frac{\partial u_{z}}{\partial z} \\
\gamma_{z r}=\frac{\partial u_{r}}{\partial z}+\frac{\partial u_{z}}{\partial r}
\end{array}\right.
$$

It is easier to get $\varepsilon_{r}$ and $\varepsilon_{\theta}$ since we could get $u_{r}$ vs. r plot from both finite element result and experiment. Here the FEM results are used to verify the kinematic relation, in which:

$$
\left\{\begin{array}{c}
\left.\varepsilon_{r}\right|_{n}=\frac{u_{r}^{n+1}-u_{r}^{n}}{r^{n+1}-r^{n}} \\
\left.\varepsilon_{\theta}\right|_{n}=\frac{u_{r}^{n}}{r^{n}}
\end{array}\right.
$$

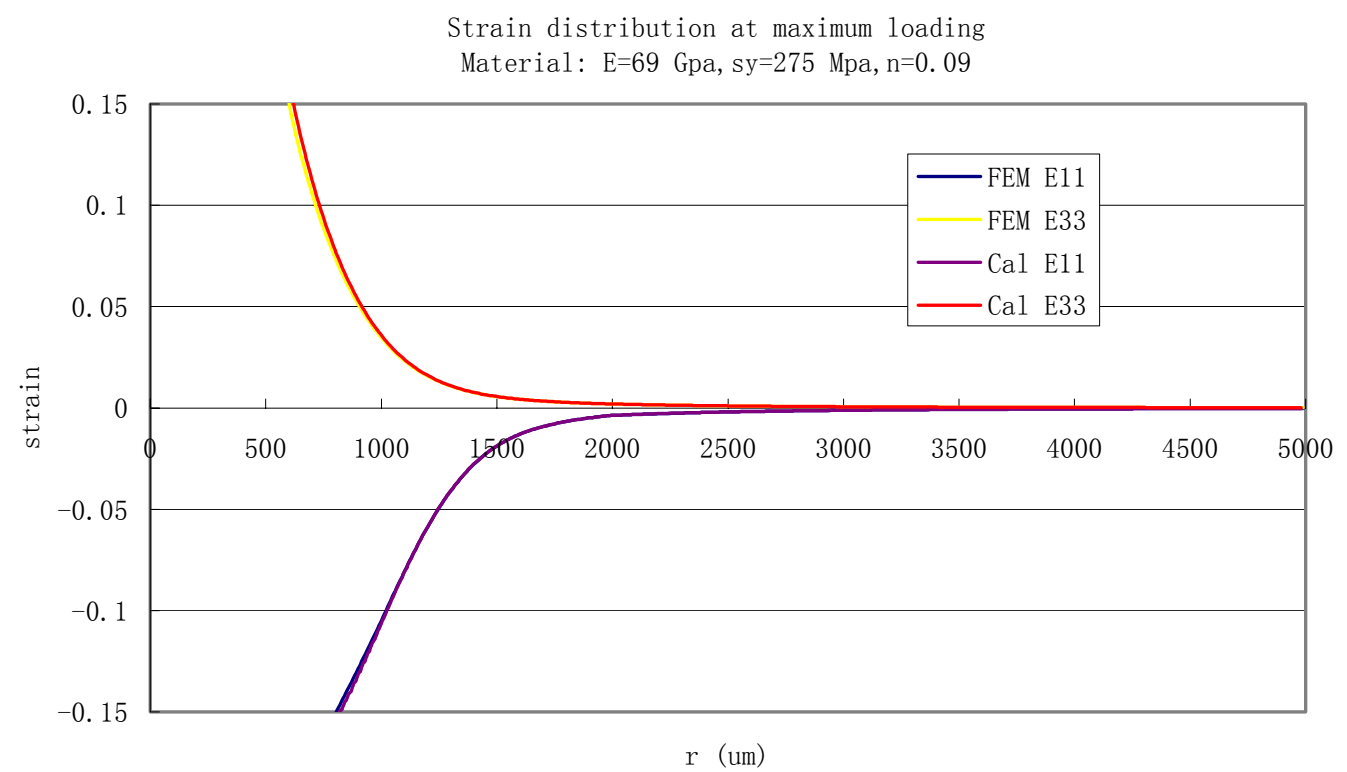

Figure 6.4.3 Strain components E11 and E33 distribution at maximum loading, where

$$
E 11=\varepsilon_{r}, E 33=\varepsilon_{\theta} .
$$


Results showed good agreement between the calculated strain components from Equation (6.5) and those given directly from FEM.

It is difficult to get $\varepsilon_{z}$ directly since it is partial derivative relative to z dimension. However, we can use elastic and plastic information to derive $\varepsilon_{z}$ from known $\varepsilon_{r}$ and $\varepsilon_{\theta}$. In elastic zone, the stress strain relation complies with Hooke's law. Plane stress condition exists on top free surface $\left(\sigma_{z}=0\right)$. So:

$\varepsilon_{z}=-\frac{v}{1-v}\left(\varepsilon_{r}+\varepsilon_{\theta}\right)$, elastic zone

In plastic zone, incompressibility condition is assumed so that

$\varepsilon_{k k}=\varepsilon_{r}+\varepsilon_{\theta}+\varepsilon_{z}=0$. Therefore:

$\varepsilon_{z}=-\left(\varepsilon_{r}+\varepsilon_{\theta}\right)$, plastic zone

The results are compared with real FEM data.

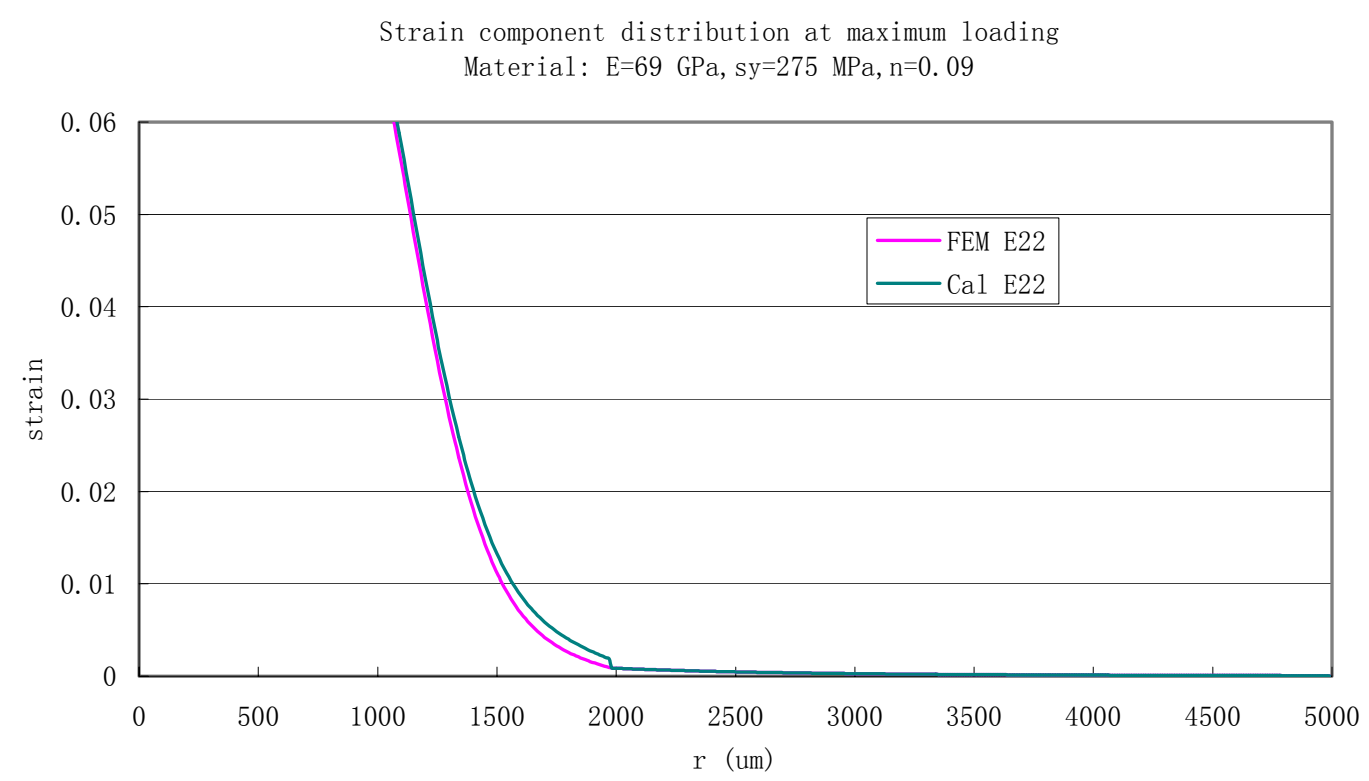

Figure 6.4.4 Strain components E22distribution at maximum loading, where $E 22=\varepsilon_{z}$. 
It is found that good agreement is achieved for elastic zone and large deformation zone (fully plasticity). Relatively large discrepancy is on the vicinity of elastic-plastic boundary.

\subsubsection{Total strain}

The effective plastic strain $\varepsilon_{e}^{p}$ can be extracted directly from FEM results. The relationship between the $\varepsilon_{e}^{p}$ and total strain in the input stress strain curve is:

$\varepsilon_{t}=\varepsilon_{e}^{p}+\frac{\sigma_{V M}}{E}$

Assume the strain components are available from displacement fields; it is necessary to develop a formula to calculate total strain from these components. The definition of von Mises stress for the plane stress problem is:

$\sigma_{V M}=\sqrt{\sigma_{r}{ }^{2}+\sigma_{\theta}{ }^{2}-\sigma_{r} \sigma_{\theta}}$

Then the equivalent total strain (assume pure elastic) is:

$\varepsilon_{t}=\frac{\sigma_{V M}}{E}=\sqrt{\left(\frac{\sigma_{r}}{E}\right)^{2}+\left(\frac{\sigma_{\theta}}{E}\right)^{2}-\frac{\sigma_{r}}{E} \frac{\sigma_{\theta}}{E}}$

From Hooke's law we have:

$$
\left\{\begin{array}{l}
\sigma_{r}=\frac{E}{1-v^{2}}\left(\varepsilon_{r}+v \varepsilon_{\theta}\right) \\
\sigma_{\theta}=\frac{E}{1-v^{2}}\left(\varepsilon_{\theta}+v \varepsilon_{r}\right)
\end{array}\right.
$$

Plug (6.10) into (6.9), we get

$$
\varepsilon_{t}=\frac{1}{1-v^{2}} \sqrt{\left(1-v+v^{2}\right)\left(\varepsilon_{r}{ }^{2}+\varepsilon_{\theta}{ }^{2}\right)-\left(1-4 v+v^{2}\right) \varepsilon_{r} \varepsilon_{\theta}}
$$

The comparison between the FEM results (6.9) and calculated results (6.13) are shown in Figure 6.4.5. 


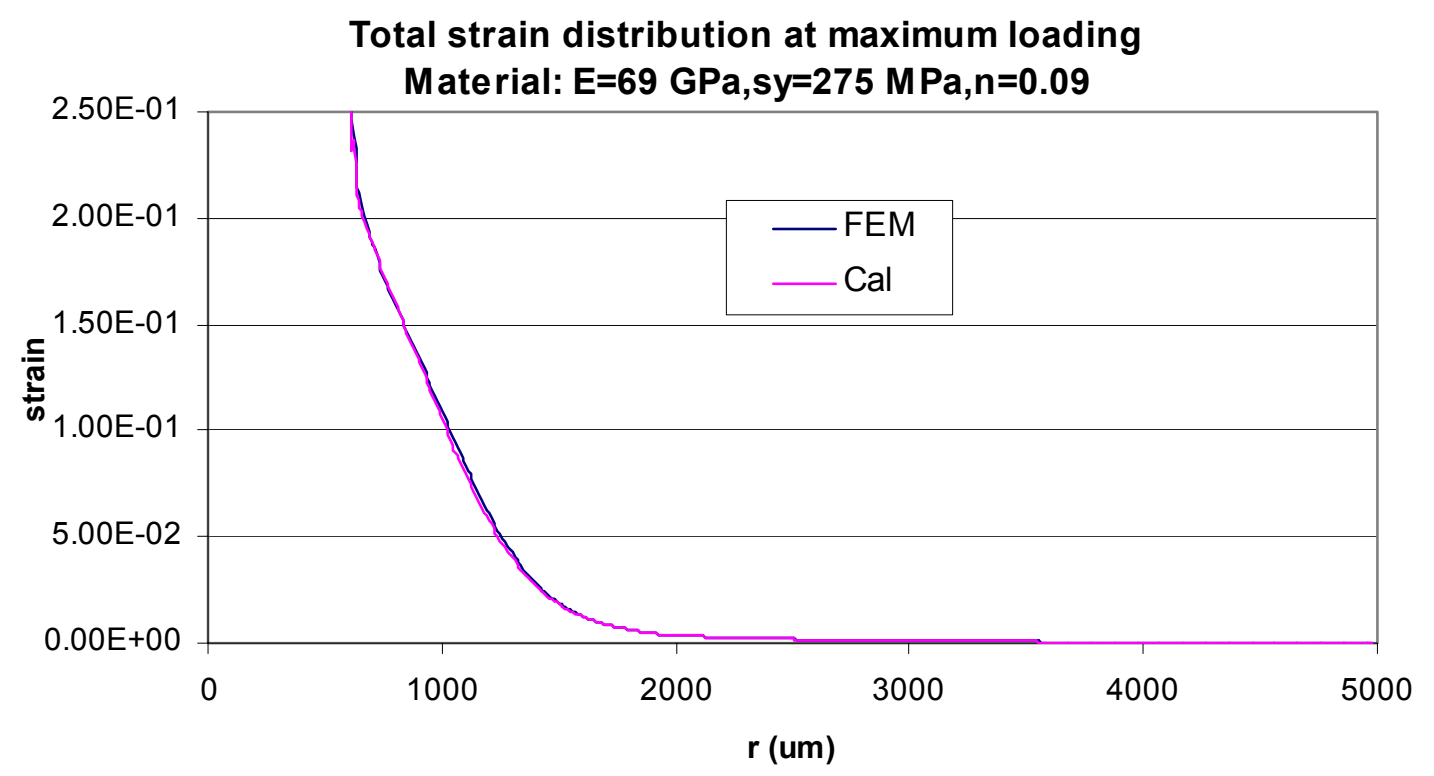

Figure 6.4.5 Total strain distribution comparisons.

The comparison results indicate perfect agreement in the elastic zone and acceptable deviation in the plastic zone. It is concluded the Equation (6.13) to calculate total strain from strain components is applicable. 


\subsection{Stress strain relation}

\subsubsection{Elastic zone and plastic zone}

The material underneath and besides the indenter is under high compressive stress. If the effective stress is higher than the yield stress, the material is in plastic deformation zone; otherwise only elastic deformation occurs. The elastic-plastic boundary is defined as the distinguishing curve of the elastic and plastic zone, on which the effective stress is the material yield stress and effective plastic strain $\varepsilon_{e}^{p}$ is zero. The elastic-plastic boundary will expand with the progressing of indentation.

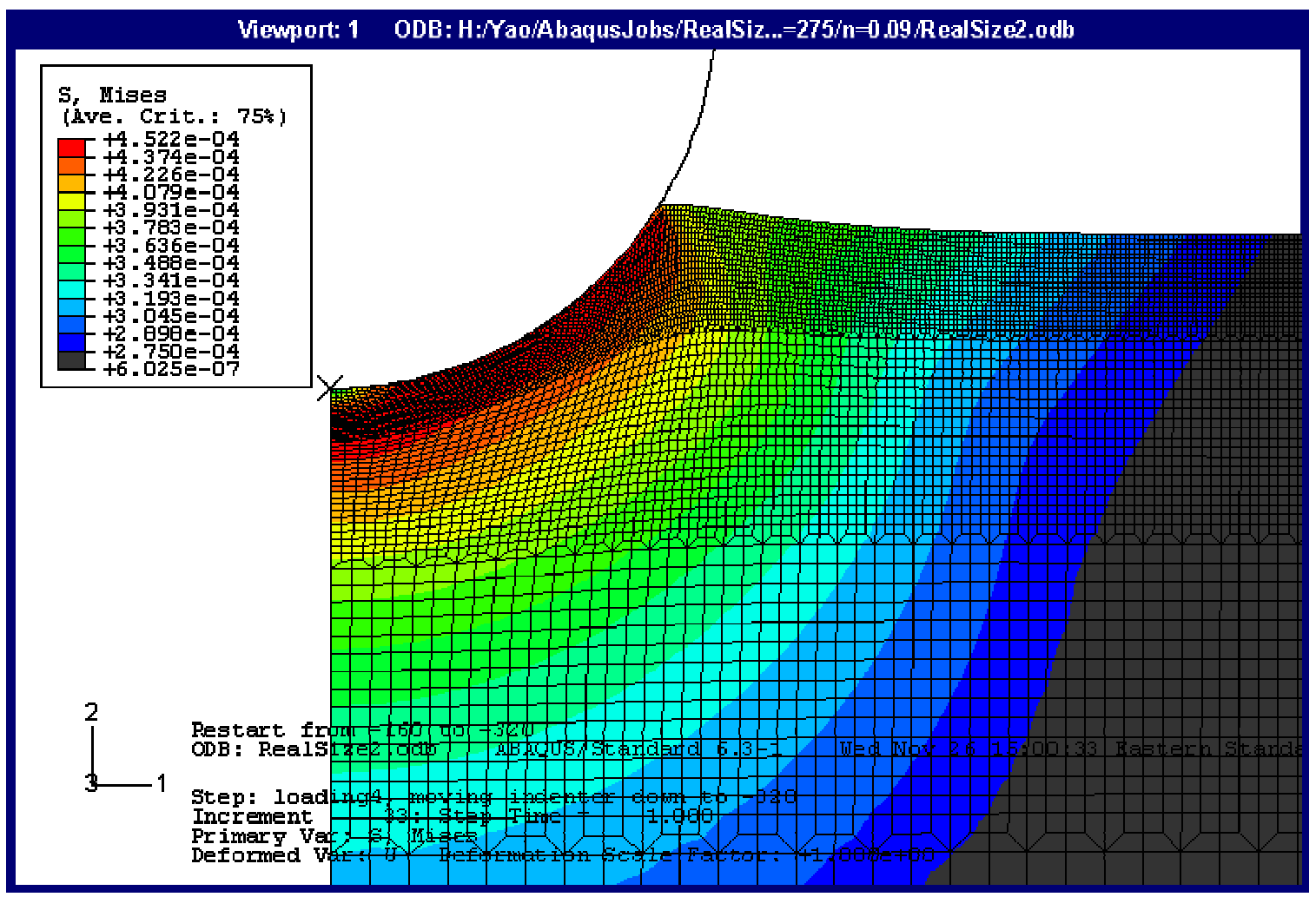

Figure 6.5.1 (a) Elastic and plastic zone for piling up material $\left(\mathrm{E}=69 \mathrm{GPa}, \sigma_{\mathrm{y}}=275 \mathrm{MPa}\right.$, $\mathrm{n}=0.09)$ at maximum loading $(320 \mu \mathrm{m})$. 


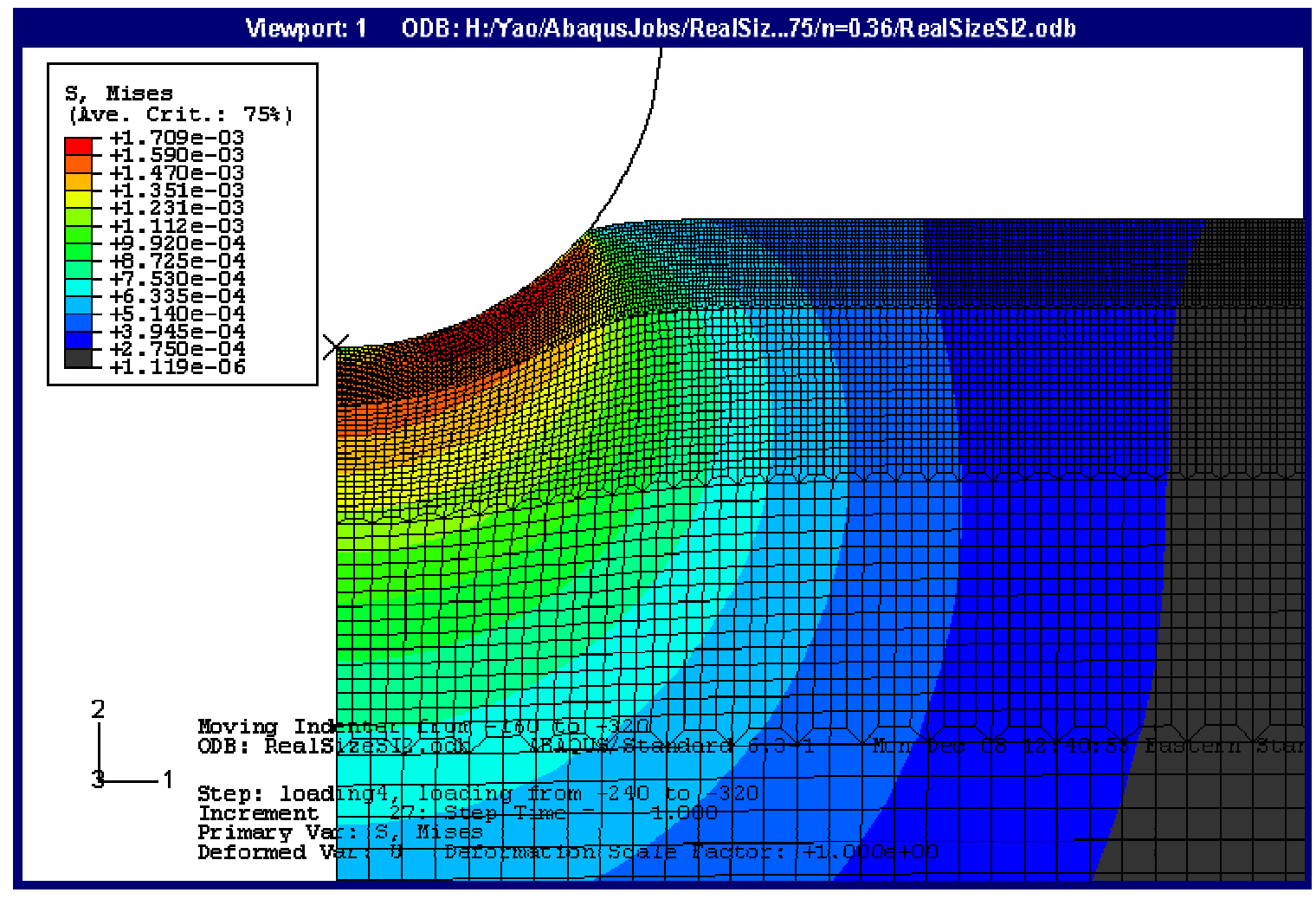

Figure 6.5.1 (b) Elastic and plastic zone for sinking in material $\left(\mathrm{E}=69 \mathrm{GPa}, \sigma_{\mathrm{y}}=275 \mathrm{MPa}\right.$, $\mathrm{n}=0.36)$ at maximum loading $(320 \mu \mathrm{m})$.

The observation of above graphs leads to the conclusion that the plastic zone is quite limited and only occupy $2 \sim 3$ times of the contact area. So, indentation is very localized procedure.

\subsubsection{Stress strain relation (single node tracing for loading process)}

Similar to the displacement field, same two nodes' (3260 and 3320) von Mises stresses are traced through the loading process. 
Single node von Mises stress variation for loading process

Material: $E=69$ GPa,sy=275 MPa,n=0.09

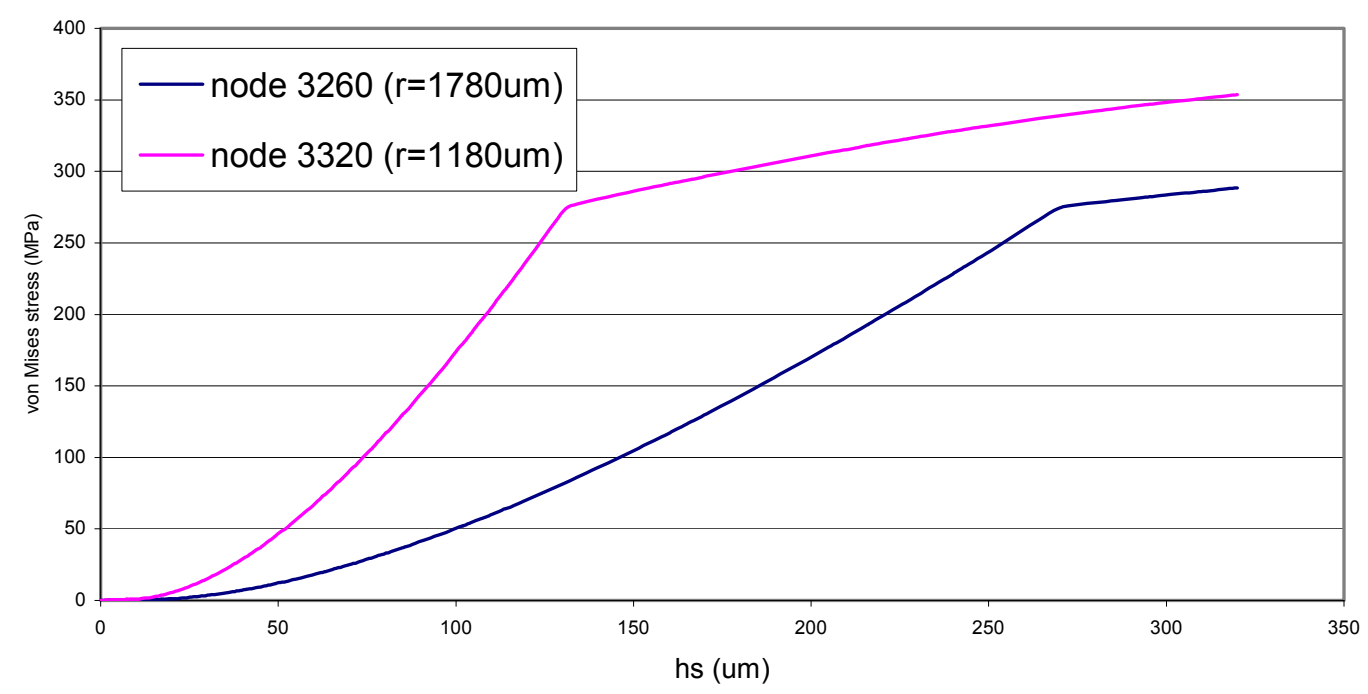

Figure 6.5.2 Two nodes von Mises stress tracing for loading process.

It is found the von Mises stress increases with the loading depth increases. The initial part of the curve is steep since that is the elastic response of the material. Hooke's law is the constitutive relation that should be complied with in this part. With the progressing of indentation, the elastic-plastic boundary will expand. When the elasticplastic boundary reaches and passes through this node, the von Mises stress increasing slows down with the increasing of indentation depth. This point of location enters plastic deformation zone. In this part, flow rule of plasticity dominates the deformation-stress relationship. The von Mises stress of the transition point from elastic to plastic deformation is the material yield stress. Also the farther the node location is from the indenter, the later the node enters plastic zone. In Figure 6.5.2, it is indicated that when $\mathrm{hs}=133 \mu \mathrm{m}$, the elastic-plastic boundary reaches node 3320 position $(\mathrm{r}=1180 \mu \mathrm{m})$. When $\mathrm{hs}=275 \mu \mathrm{m}$, the boundary reaches node 3260 position $(\mathrm{r}=1780 \mu \mathrm{m})$. 
The total strain $\varepsilon_{t}=\varepsilon_{e}^{p}+\frac{\sigma_{V M}}{E}$ could be calculated through its numerical results for the same nodes (3260 and 3320). Then the total strain vs. von Mises stress could be plotted as:

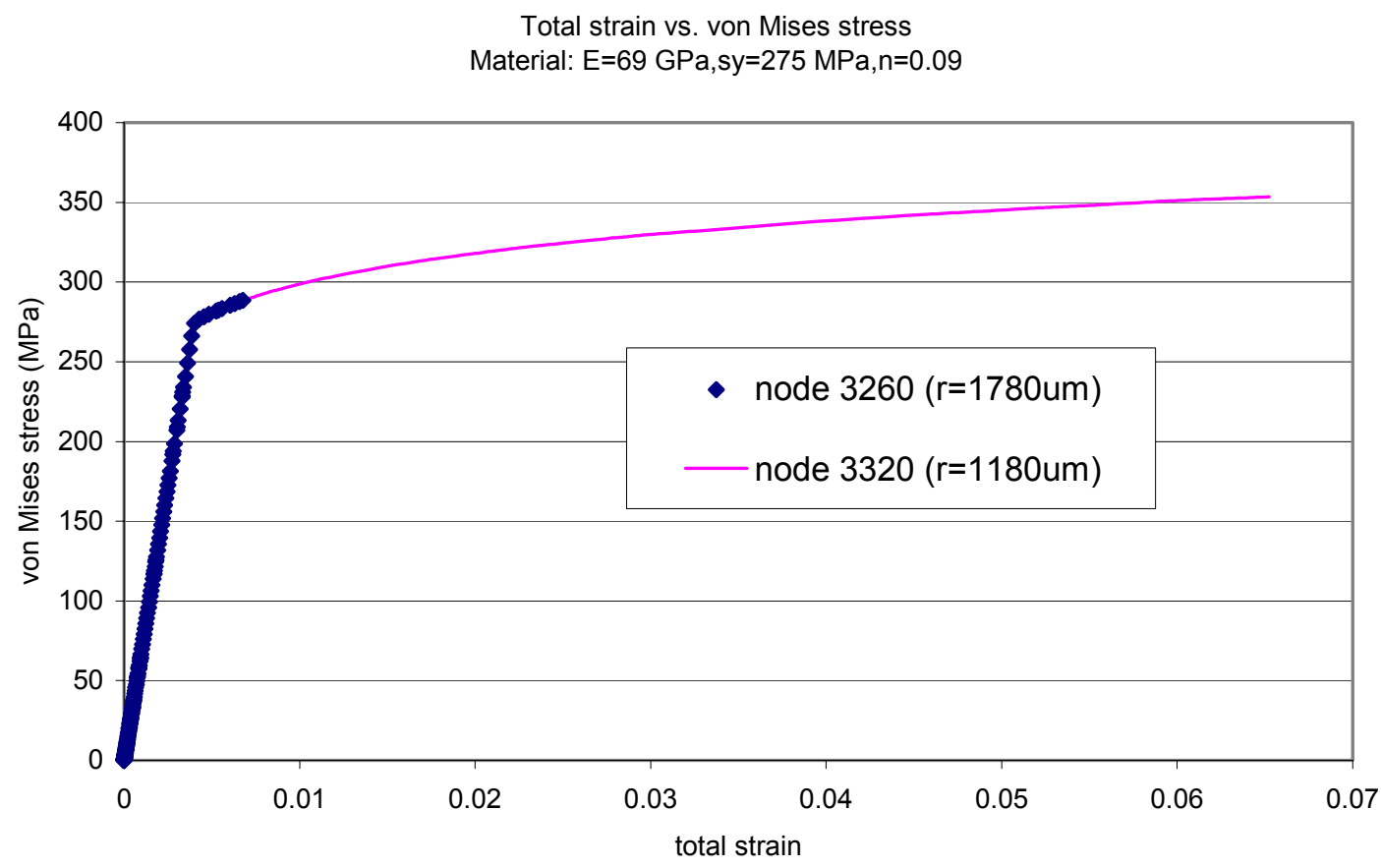

Figure 6.5.3 Total strain vs. von Mises stress by tracing single node for loading process.

It is found that for each node, its total strain (equation) and von Mises equivalent stress relation for the loading process is exactly the input stress strain curve. Each node experienced same stress strain history that is governed by uniaxial stress-strain relationship of the observed material. The difference among them is the extent going into plastic deformation. At the same loading status, the farther the node is, the less plastic deformation it is subjected to. That explains that in Figure 6.5.3 the node 3320 (location $\mathrm{r}=1180 \mu \mathrm{m}$ ) has a much larger plastic strain comparing with node 3260 (location $\mathrm{r}=1780$ $\mu \mathrm{m})$.

6.5.3 Stress strain relation (surface contour at certain loading status) 
The von Mises stress varies on different location on surface at certain loading status. After unloading, there is still stress existing which is called residual stress.

In Section 6.4, the displacement fields and strain fields at both maximum loading and unloading status have been extracted. The stress strain relationship in the elastic zone is governed by Hooke's law, which is:

$$
\left\{\begin{array}{l}
\sigma_{r}=\frac{E}{1-v^{2}}\left(\varepsilon_{r}+v \varepsilon_{\theta}\right) \\
\sigma_{\theta}=\frac{E}{1-v^{2}}\left(\varepsilon_{\theta}+v \varepsilon_{r}\right)
\end{array}\right.
$$

and $\sigma_{z}=0$ on the surface of half space which indicate plane stress condition on surface. $\sigma_{r}, \sigma_{\theta}, \sigma_{z}$ are three principle stresses. Written in the form of $\sigma_{1} \geq \sigma_{2} \geq \sigma_{3}$, it becomes: $\left\{\begin{array}{c}\sigma_{1}=\sigma_{z}=0 \\ \sigma_{2}=\sigma_{\theta} \\ \sigma_{3}=\sigma_{r}\end{array}\right.$ or $\left\{\begin{array}{c}\sigma_{1}=\sigma_{\theta} \\ \sigma_{2}=\sigma_{z}=0, \text { depending on the observing location. The absolute } \\ \sigma_{3}=\sigma_{r}\end{array}\right.$ value of $\sigma_{r}$ is always larger than that of $\sigma_{\theta}$. This conclusion is also confirmed by current FEM simulation result. 


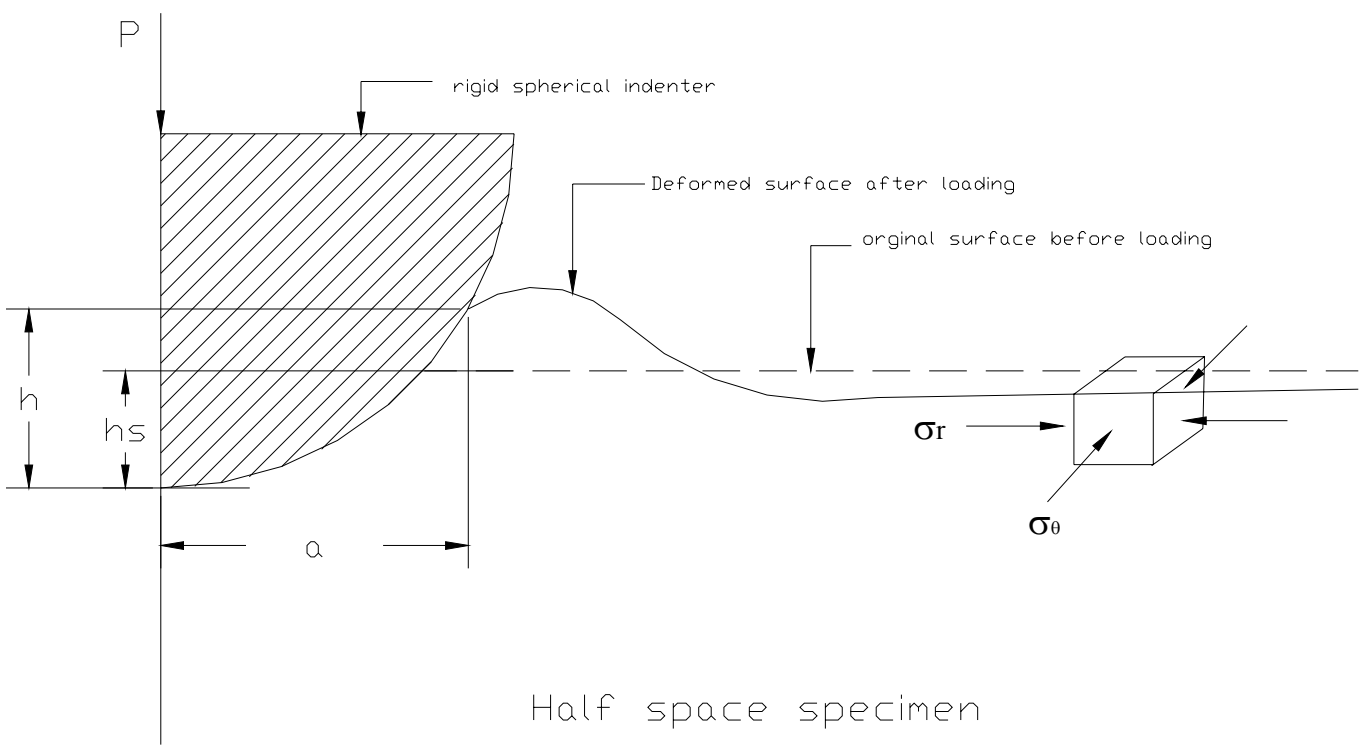

Figure 6.5.4 Schematic drawing of surface state of stress for piling up material.

So, from definition, von Mises stress is:

$\sigma_{V M}=\sqrt{3 J_{2}}=\sqrt{\sigma_{r}{ }^{2}+\sigma_{\theta}{ }^{2}-\sigma_{r} \sigma_{\theta}}$

Through this process, the von Mises stress could be calculated from strain fields. Then the calculation results are compared with the results extracted directly from FEM simulation. 


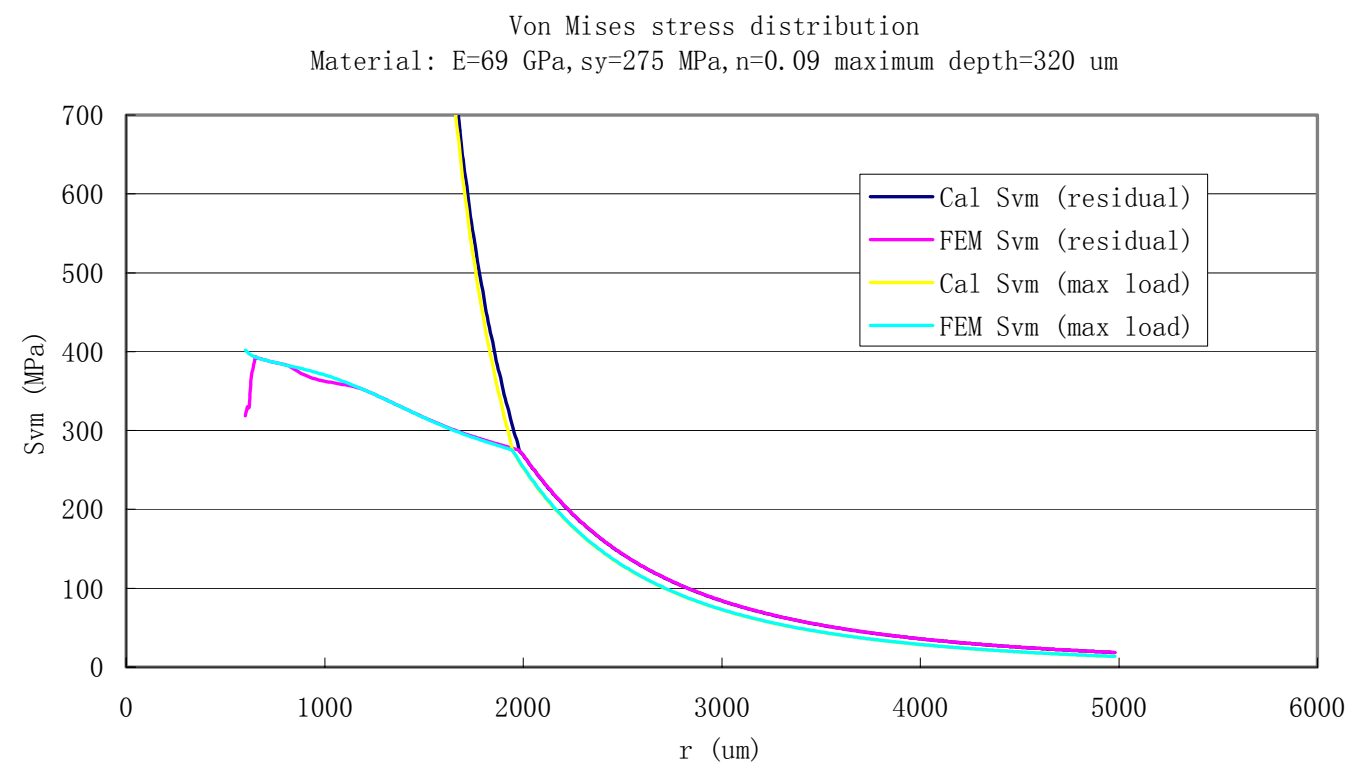

Figure 6.5.5 Von Mises stress distribution at both maximum loading and unloading status.

It is concluded from the above plot that:

1. The calculation of von Mises stress from strain fields using Hooke's law could only be applied in elastic zone. Very good agreement of the comparison between them is reached.

2. For plastic zone, the calculated strain is large due to the plastic flow of the material. The error between the Hooke's law calculation and real results is getting larger.

3. The deviation between the Hooke's law calculation and real FEM occurres at elastic-plastic boundary, at which point the von Mises stress corresponds to yield stress. So, once the position of the elastic-plastic boundary could be determined by W field variation (see Section 6.4), the yield stress could be calculated. 
4. The von Mises stress distribution does not change much from the maximum loading status to totally unloading status. The elastic-plastic boundary even gets farther from the indenter when the load is totally removed.

Also the surface total strain field was discussed in detail in Section 6.4. Each point on surface experiences the same stress strain variation history that is dominated by input material model. The points, which are close to indenter, experience larger strain while those far from indenter experienced small strain. So the von Mises effective stress vs. total effective strain curve is plotted through the surface contour:

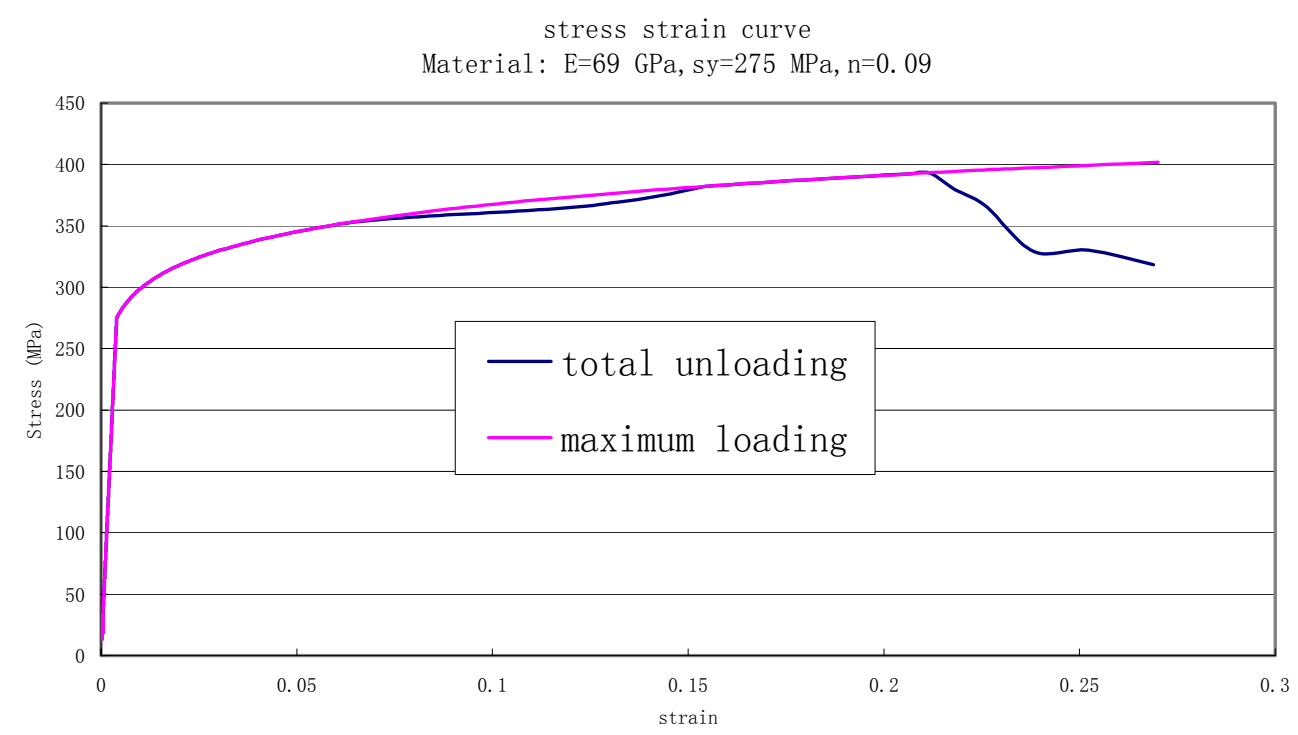

Figure 6.5.6 Stress strain curve obtained from surface von Mises stress and total strain fields.

It is found that the stress strain relationship for maximum loading perfectly agrees with the input stress strain curve. For the unloading curve, some deviation occurs in relatively large deformation area. 


\subsection{Elastic rebound during unloading and cyclic reloading}

\subsubsection{Elastic rebound during unloading}

As shown before, the load removal leads to elastic rebound resulting in an upward displacement of material at the indented surface (along the $\mathrm{z}$ direction), the surface profiles in the unloaded state would be expected to exhibit an increased tendency to piling-up. This process is assumed to be pure elastic rebound and only related to the elastic properties of the material. According to this assumption, Hertz analytical solution could be employed to determine the Young's modulus from the W field variation during this process. In this part, the finite element results would be extracted to compare with the analytical solution to verify and develop this method.

The $\mathrm{W}$ displacement during this elastic process could be obtained by calculating the $\mathrm{W}$ displacement difference between residual and maximum loading status (in these

cases, hs $=320 \mu \mathrm{m})$ from FEM simulation results, which is $W=W_{\text {residual }}-W_{\max \text { Load }}$. The Equation (5.3) provides calculation of Hertz solution. Two materials, one is typical piling-up $(\mathrm{n}=0.09)$ and one is typical sinking-in $(\mathrm{n}=0.36)$, are examined. 
Unloading W field comparison between FEM and analytical results

Material: $\mathrm{E}=69 \mathrm{GPa} \mathrm{sy}=275 \mathrm{MPa} \mathrm{n}=0.09$

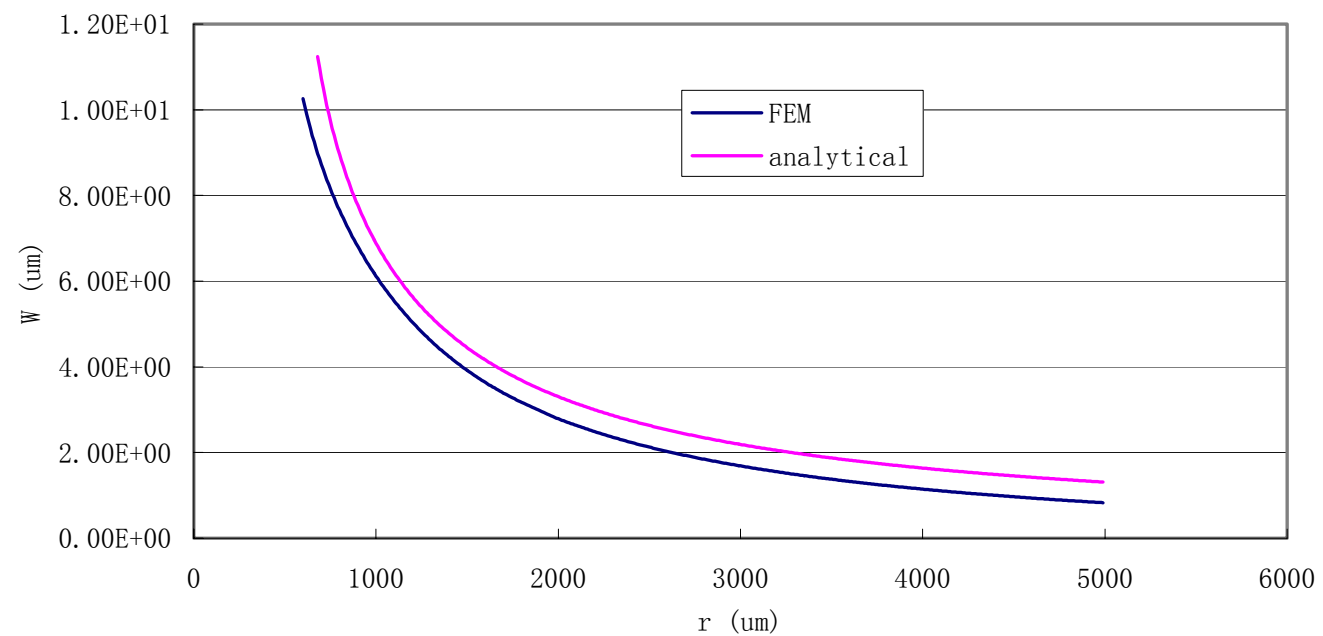

Figure 6.6.1 (a) Unloading W field comparison between FEM and analytical results (piling up material, $\mathrm{n}=0.09$ ).

W field comparison between FEM and analytical results

Material: $\mathrm{E}=69 \mathrm{GPa}$ sy=275 $\mathrm{MPa} \mathrm{n}=0.36$

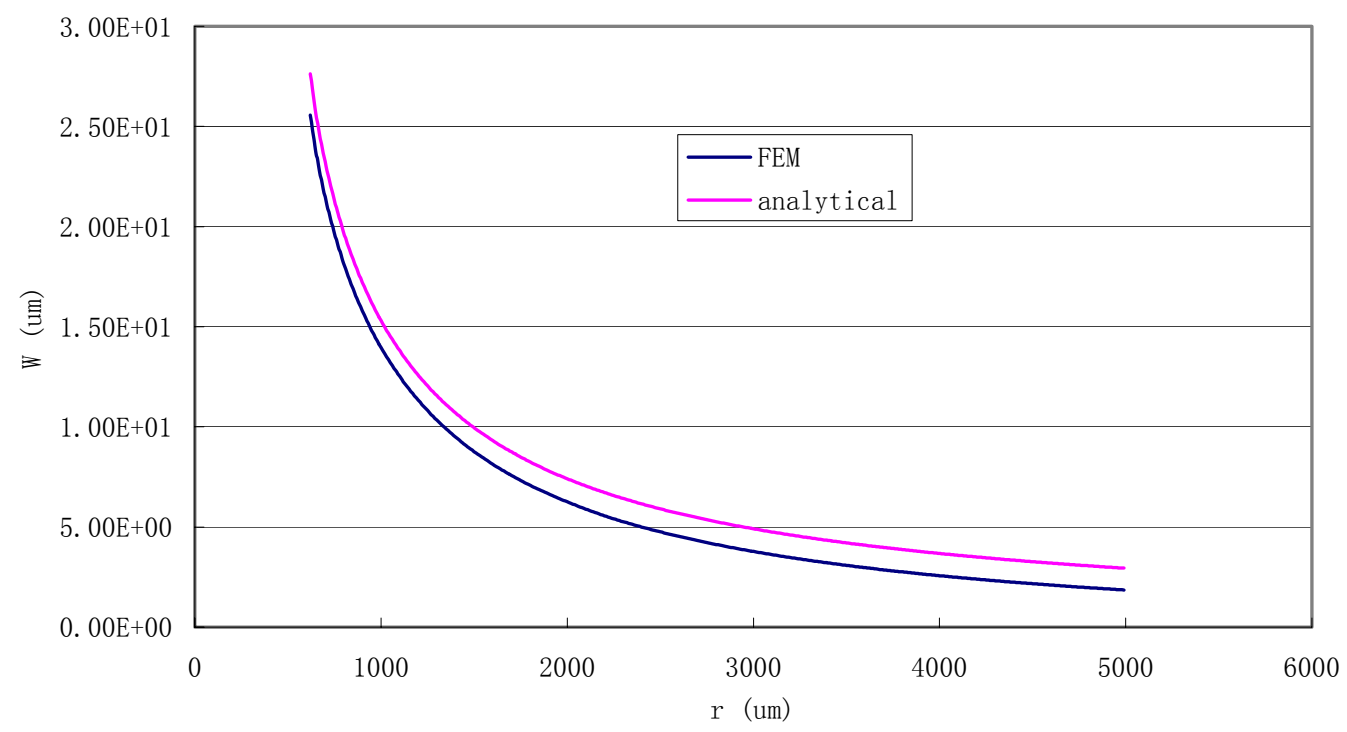

Figure 6.6.1 (b) Unloading W field comparison between FEM and analytical results

(sinking in material, $\mathrm{n}=0.36$ ). 
Both Figures indicate a disagreement between the FEM results and Hertz analytical solution. This difference is expected and reasonable since the datum of displacements in the analytical solution is original flat surface while that in FEM results is deformed contour at residual status. Also it is observed an almost constant difference exists for both materials. The quantity of the constant is related to the amount of surface deformation (piling up or sinking in).

The observation of same variation trend between FEM and analytical solution in the Figure 6.6.1 (a) and (b) provides us an assumption that first derivative should be consistent between them, which will cancel the influence of the constant mentioned above.

For analytical solution, take the first differentiation of equation (5.3):

$$
\frac{d u_{z}}{d r}=\frac{3 p_{m}\left(1-v^{2}\right)}{4 a E}\left[-2 r \sin ^{-1} \frac{a}{r}+a\left(1-2 \frac{a^{2}}{r^{2}}\right)\left(1-\frac{a^{2}}{r^{2}}\right)^{-1 / 2}+a\left(1-\frac{a^{2}}{r^{2}}\right)^{1 / 2}+a \frac{a^{2}}{r^{2}}\left(1-\frac{a^{2}}{r^{2}}\right)^{-1 / 2}\right]
$$

In FEM, using $\left.\frac{d u_{z}}{d r}\right|_{n}=\frac{u_{z}^{n+1}-u_{z}^{n}}{r^{n+1}-r^{n}}$ to calculate derivative at certain point and connect the points as a curve. 


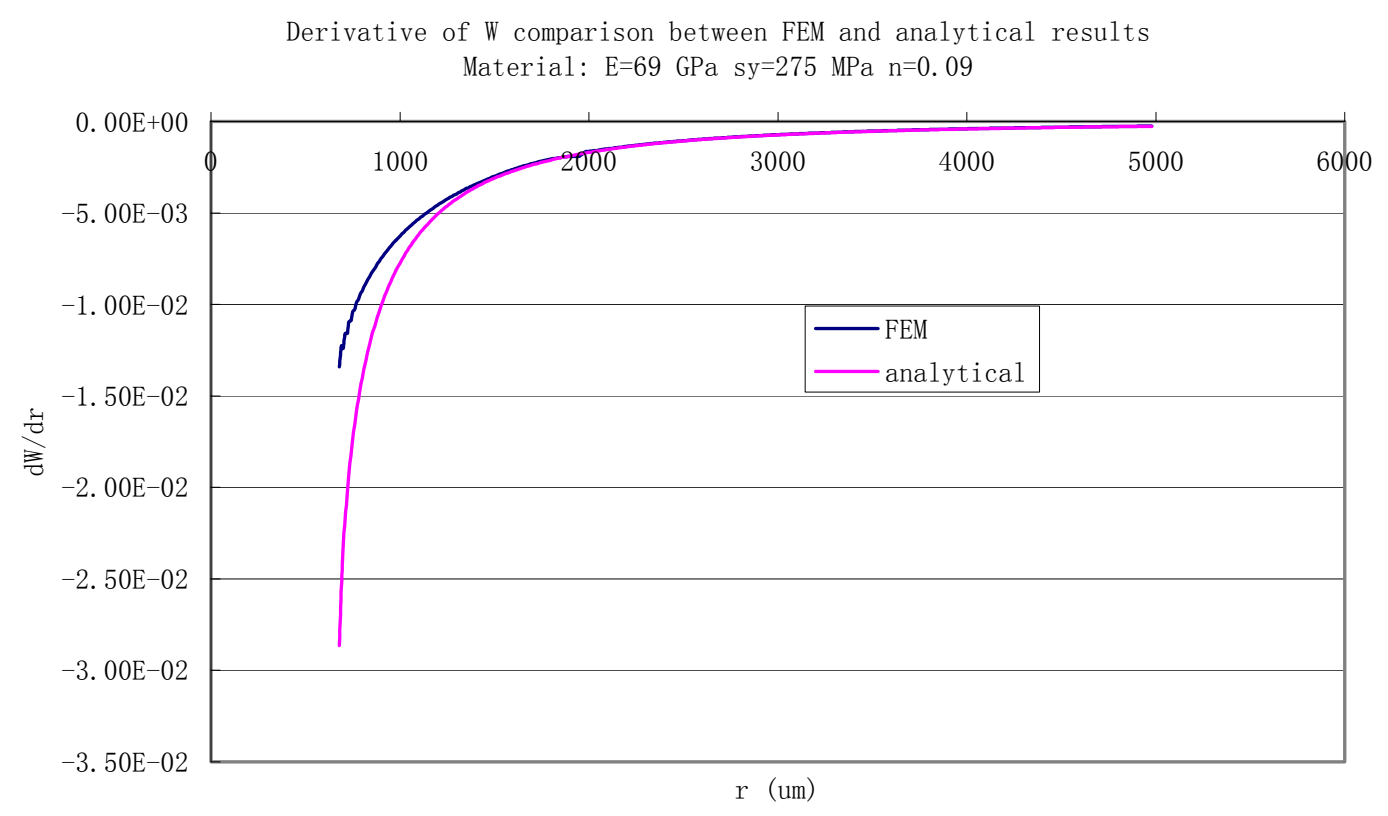

Figure 6.6.2 (a) Derivative of W comparison between FEM and analytical results (Piling up material, $\mathrm{n}=0.09$ ).

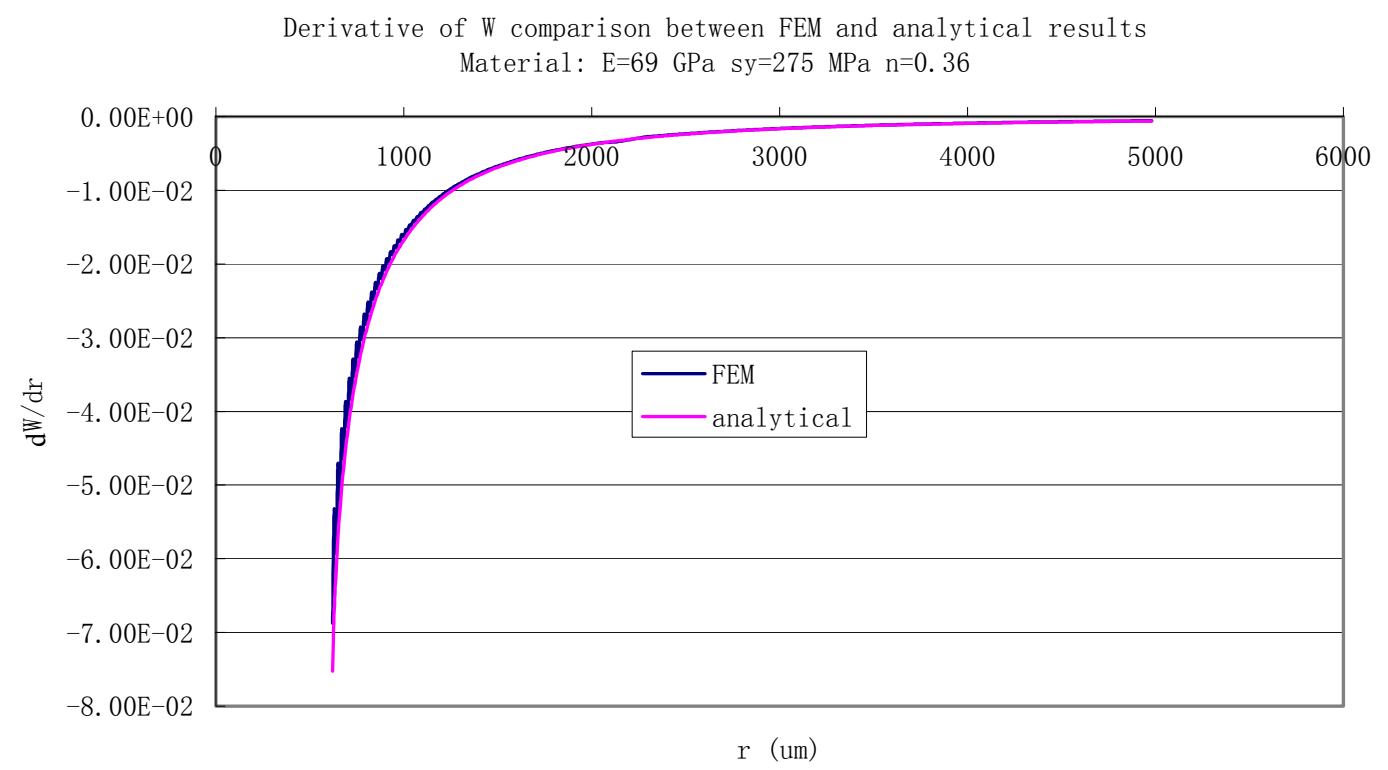

Figure 6.6.2 (b) Derivative of W comparison between FEM and analytical results (Sinking in material, $\mathrm{n}=0.36$ ). 
The results indicate a good agreement between the FEM simulation results and analytical solution, especially for the elastic deformation area (far from the indenter). It is also speculated that the shallower the indentation is, the better the agreement should be observed due to less plastic deformation influence and better agreement with small deformation assumption in Hertz theory.

This discovery provides an alternative method to extract material Young's modulus from $\mathrm{W}$ displacement field, which could be experimentally obtained from optical method (interferometry).

Another observation directly from finite element simulation is that the contact area will keep almost constant during initial unloading. Then the contact between the indenter and half space will start to separate gradually starting from the top with the decrease of load until the total separation of contact when the load is zero. This observation supports the former assumption of constant contact area during initial unloading and thus validates the methods to calculate elastic properties from instrumented indentation tests.

\subsubsection{Cyclic reloading}

It has already been noted that the permanent indentation left in a metal surface deformed by a hard spherical indenter has a larger radius of curvature than that of the indenting sphere. This effect has generally been ascribed to the release of elastic stresses in the specimen. Now that the unloading process is pure elastic rebound, it should be essentially reversible.

One material model (Aluminum 6061-T6, E=69 GPa, $\sigma_{\mathrm{y}}=275 \mathrm{MPa}$ and $\mathrm{n}=0.09$ ) is employed to perform the cyclic loading finite element simulation. The loading process 
is: load to hs=160 $\mu \mathrm{m}$, unloading to $150 \mu \mathrm{m}$, reloading to $160 \mu \mathrm{m}$ and continue loading to $320 \mu \mathrm{m}$, then unloading to $300 \mu \mathrm{m}$. Load depth curve of current FEM simulation is compared with previously extracted load depth curve in which the indentation is loaded to hs $=320 \mu \mathrm{m}$ directly and then unloading to $300 \mu \mathrm{m}$.

Direct loading and cyclic loading comparison (Material model: E=69 GPa, sy=275 MPa, n=0.09)

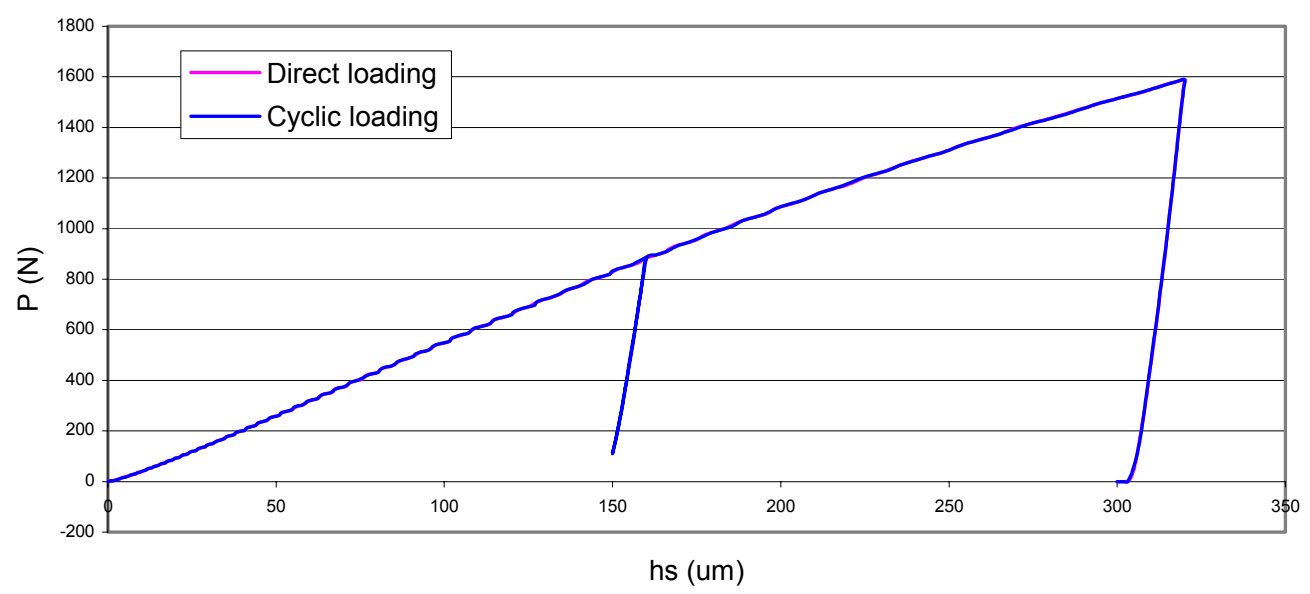

Figure 6.6.3 Load depth curve comparison: direct loading and cyclic loading.

As is found in Figure 6.6.3, the unloading from $160 \mu \mathrm{m}$ to $150 \mu \mathrm{m}$ is totally and perfectly reversible, indicating the unloading is pure elastic rebound of material. Thus the former methods to extract elastic modulus from initial unloading are validated. Afterwards loading and unloading curve is perfectly overlapping with that of direct loading process. So the midway unloading will generate no effect on afterwards mechanical behavior of the material in indentation tests. 


\subsection{Strain hardening and Meyer's law}

The key relation between contact radius (a), indenter diameter (D) and applied load (P) was proposed by Meyer, which governs spherical indentation, namely:

$$
P=K \frac{a^{n+2}}{D^{n}}
$$

where $\mathrm{n}$ and $\mathrm{K}$ are material constants. Over the years Equation (6.16) became known as Meyer's law. This equation can be rearranged as

$$
H=p_{m}=\frac{P}{\pi a^{2}}=\kappa\left(\frac{a}{D}\right)^{n}
$$

where $\mathrm{H}$ is the Meyer hardness and $\kappa=K / \pi$. Further work by O'Neill and Tabor [39] showed that the value $\mathrm{n}$ from indentation tests is equal to the strain hardening exponent from the uniaxial true stress $(\sigma)$ - true plastic strain $\left(\varepsilon_{p}\right)$ curve, which of a wide variety of metals follows:

$$
\sigma=\sigma_{0} \varepsilon_{p}^{n}
$$

However, in our material model, the definition of strain hardening exponent $\mathrm{n}$ is different from above equation. In our definition:

$$
\sigma=\sigma_{y}\left(\frac{E}{\sigma_{y}}\right)^{n} \varepsilon^{n} \text { for } \sigma>\sigma_{y}
$$

Then what is the relationship between $\mathrm{P}$ and this $\mathrm{n}$ value?

At first it is assumed that $P \propto a^{f(n)}, \mathrm{f}(\mathrm{n})$ is an unknown function of $\mathrm{n}$. Now we are trying to derive an uniform $\mathrm{f}(\mathrm{n})$ function from the 21 material models.

Loading process $\log (\mathrm{a})$ vs. $\log (\mathrm{P})$ plots are plotted for the 21 material models. (contact radius a value is directly obtained from FE simulation). 
$\log (a)$ vs. $\log (P)$ plot

Material set: $E=69 \mathrm{Gpa}, \mathrm{sy}=275 \mathrm{Mpa}$

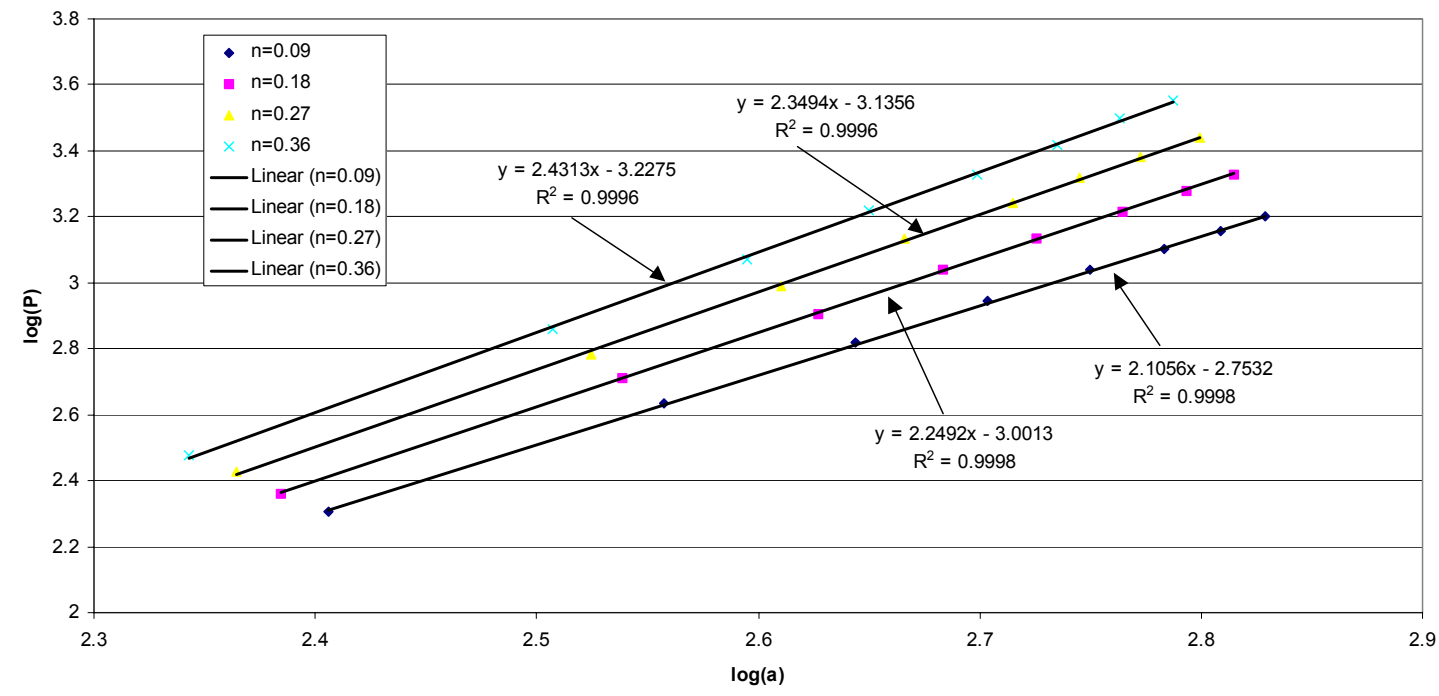

Figure 6.7.1 (a) $\log (\mathrm{a})$ vs. $\log (\mathrm{P})$ plot for material set: $\mathrm{E}=69 \mathrm{GPa}, \sigma_{\mathrm{y}}=275 \mathrm{MPa}, \mathrm{n}=0.09$, $0.18,0.27,0.36$ respectively.

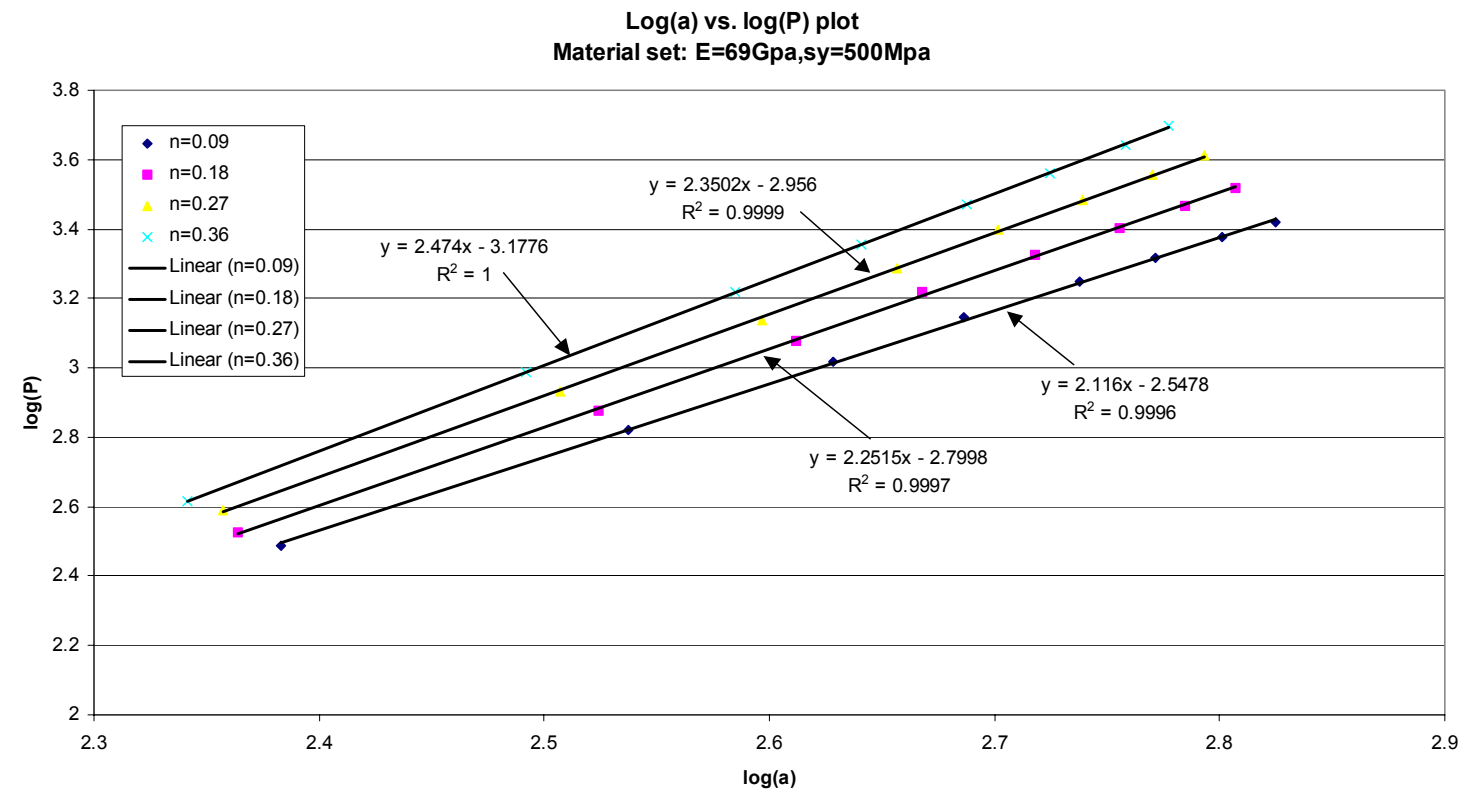

Figure 6.7.1 (b) $\log (\mathrm{a})$ vs. $\log (\mathrm{P})$ plot for material set: $\mathrm{E}=69 \mathrm{GPa}, \sigma_{\mathrm{y}}=500 \mathrm{MPa}, \mathrm{n}=0.09$, $0.18,0.27,0.36$ respectively. 


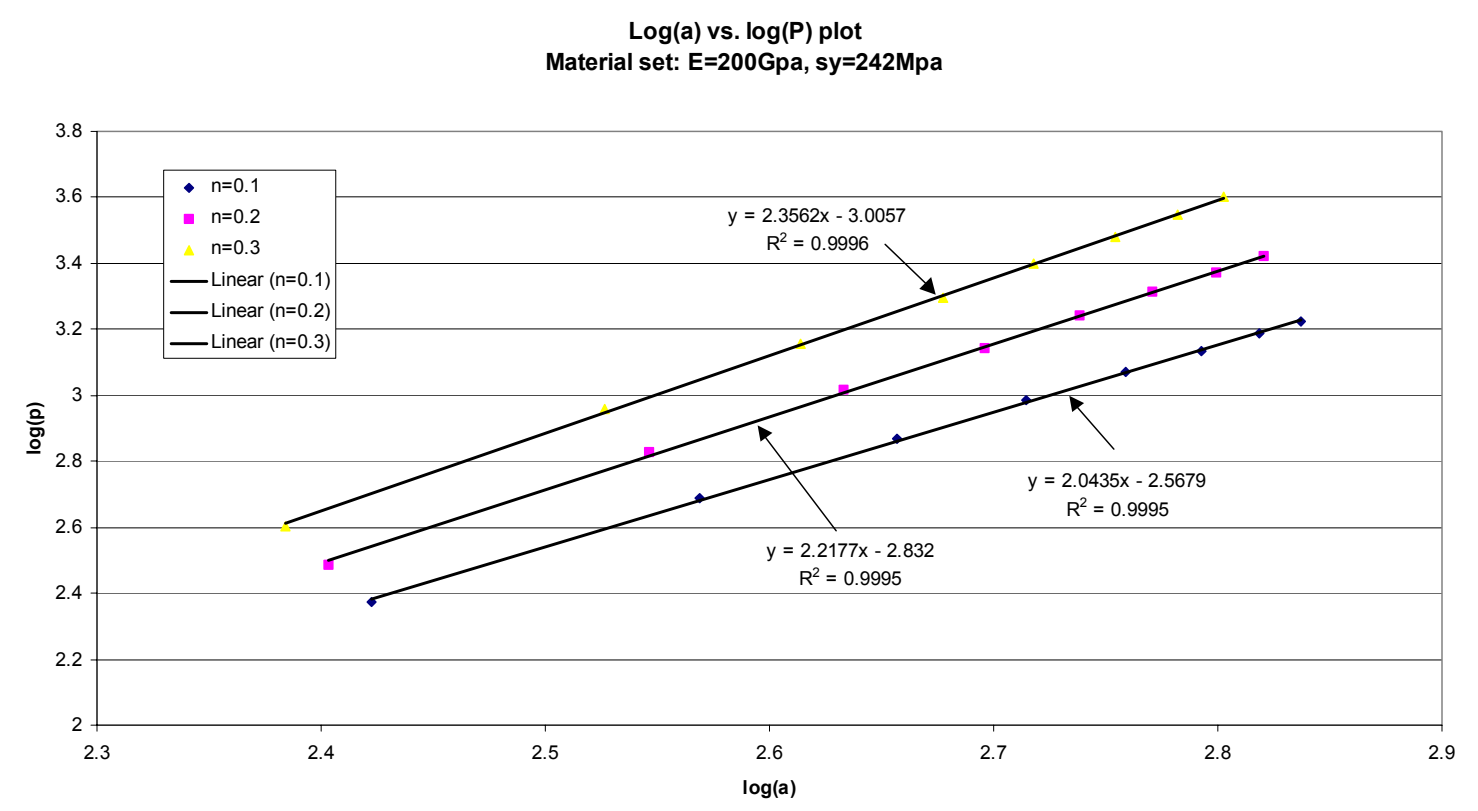

Figure 6.7.1 (c) $\log (\mathrm{a})$ vs. $\log (\mathrm{P})$ plot for material set: $\mathrm{E}=200 \mathrm{GPa}, \sigma_{\mathrm{y}}=242 \mathrm{MPa}, \mathrm{n}=0.1$, $0.2,0.3$ respectively.

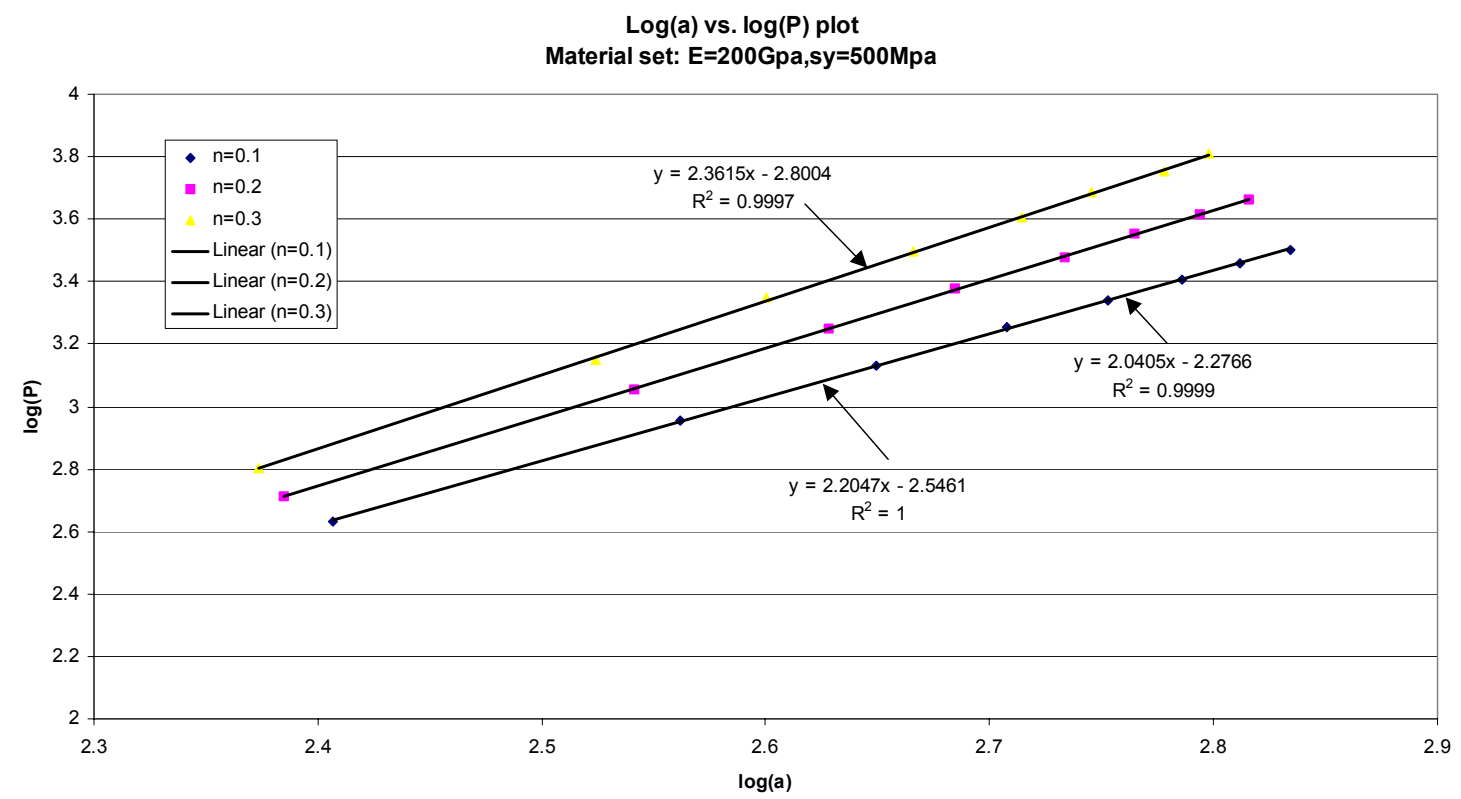

Figure 6.7.1 (d) $\log (\mathrm{a})$ vs. $\log (\mathrm{P})$ plot for material set: $\mathrm{E}=200 \mathrm{GPa}, \sigma_{\mathrm{y}}=500 \mathrm{MPa}, \mathrm{n}=0.1$, $0.2,0.3$ respectively. 


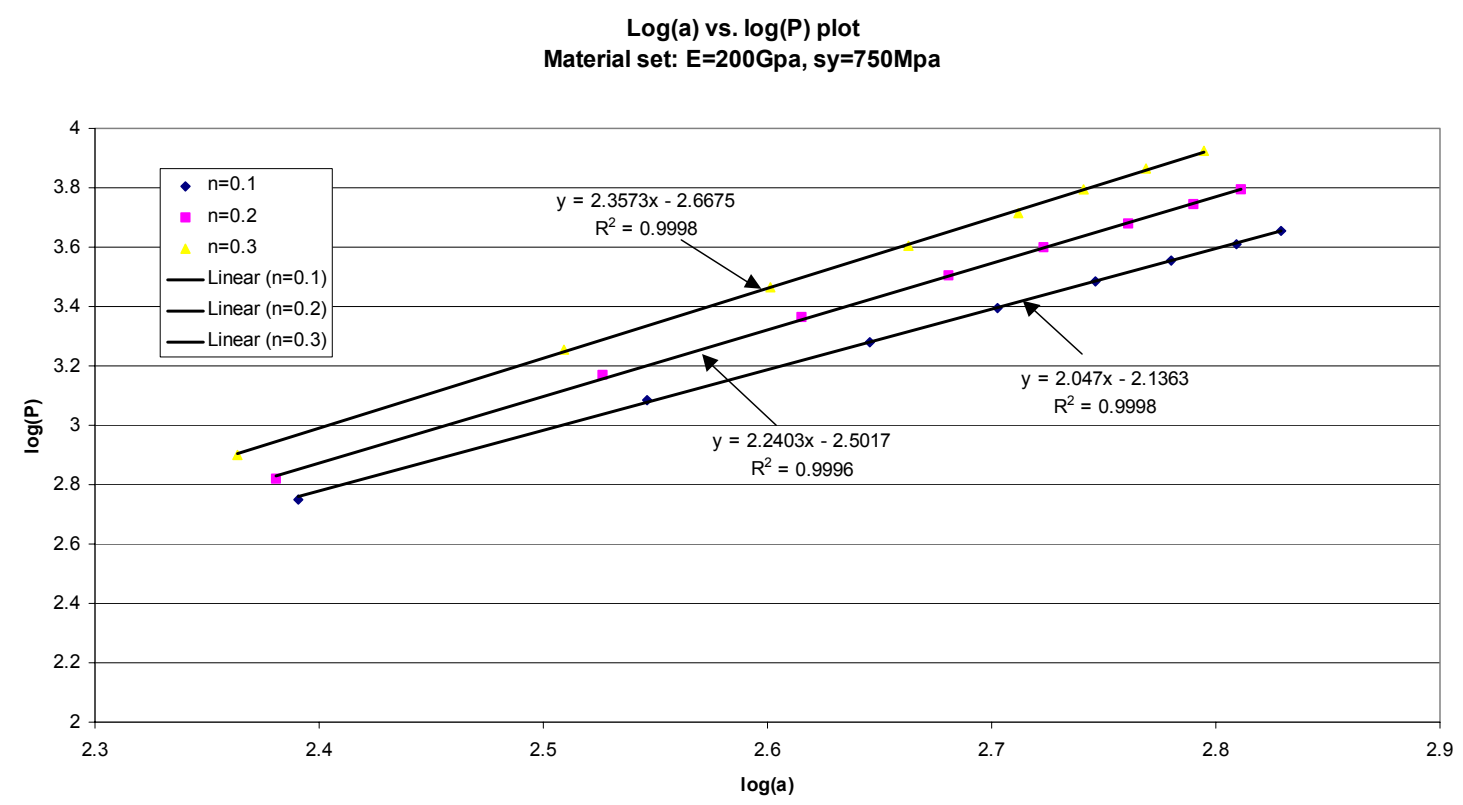

Figure 6.7.1 (e) $\log (\mathrm{a})$ vs. $\log (\mathrm{P})$ plot for material set: $\mathrm{E}=200 \mathrm{GPa}, \sigma_{\mathrm{y}}=750 \mathrm{MPa}, \mathrm{n}=0.1$, $0.2,0.3$ respectively.

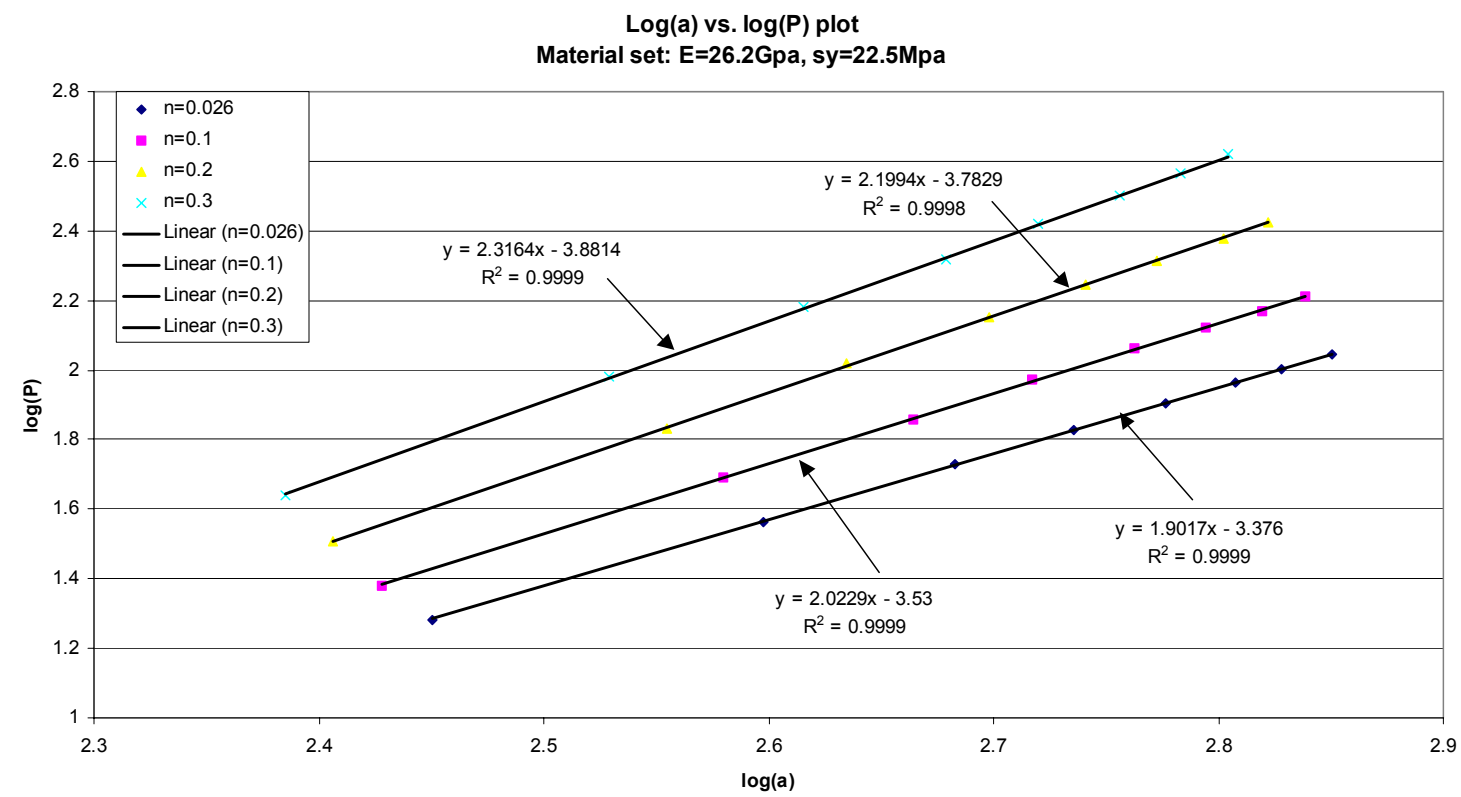

Figure 6.7.1 (f) $\log (\mathrm{a})$ vs. $\log (\mathrm{P})$ plot for material set: $\mathrm{E}=26.2 \mathrm{GPa}, \sigma_{\mathrm{y}}=22.5 \mathrm{MPa}$, $\mathrm{n}=0.026,0.1,0.2,0.3$ respectively. 
The $\log (\mathrm{a}) \mathrm{vs} . \log (\mathrm{P})$ plots for all the 21 material models indicate a good linear relationship so that linear curve fittings are made to extract the slope of the straight line, which is essentially represented by $f(n)$. The results are listed in the table below:

Table 6.3 Slope of $\log (\mathrm{a})$ vs. $\log (\mathrm{P})$ plot

\begin{tabular}{|c|c|c|c|c|}
\hline \multicolumn{3}{|c|}{ Material Model } & \multirow{2}{*}{$\begin{array}{c}\text { Slope of } \log (a) \text { vs. } \log (\mathrm{P}) \\
\text { plot }\end{array}$} & \multirow[b]{2}{*}{ R-squared value } \\
\hline \multirow{9}{*}{$\begin{array}{c}\text { Aluminum } \\
\text { Alloys } \\
\mathrm{E}=69 \mathrm{GPa} \\
v=0.33\end{array}$} & $\begin{array}{l}\text { Yield } \\
\text { stress }\end{array}$ & $\mathrm{n}$ & & \\
\hline & \multirow{4}{*}{$\begin{array}{c}275 \\
(\mathrm{MPa})\end{array}$} & 0.09 & 2.1056 & 0.9998 \\
\hline & & 0.18 & 2.2492 & 0.9998 \\
\hline & & 0.27 & 2.3494 & 0.9996 \\
\hline & & 0.36 & 2.4313 & 0.9996 \\
\hline & \multirow{4}{*}{$\begin{array}{c}500 \\
(\mathrm{MPa})\end{array}$} & 0.09 & 2.116 & 0.9996 \\
\hline & & 0.18 & 2.2515 & 0.9997 \\
\hline & & 0.27 & 2.3502 & 0.9999 \\
\hline & & 0.36 & 2.474 & 1 \\
\hline \multirow{9}{*}{$\begin{array}{c}\text { Steel Alloys } \\
\mathrm{E}=200 \mathrm{GPa} \\
v=0.3\end{array}$} & \multirow{3}{*}{$\begin{array}{c}242 \\
(\mathrm{MPa})\end{array}$} & 0.1 & 2.0435 & 0.9995 \\
\hline & & 0.2 & 2.2177 & 0.9995 \\
\hline & & 0.3 & 2. 3561 & 0.9996 \\
\hline & \multirow{3}{*}{$\begin{array}{c}500 \\
(\mathrm{MPa})\end{array}$} & 0.1 & 2.0405 & 0.9999 \\
\hline & & 0.2 & 2.2047 & 1 \\
\hline & & 0.3 & 2.3615 & 0.9997 \\
\hline & \multirow{3}{*}{$\begin{array}{c}750 \\
(\mathrm{MPa})\end{array}$} & 0.1 & 2.047 & 0.9998 \\
\hline & & 0.2 & 2.2403 & 0.9996 \\
\hline & & 0.3 & 2.3573 & 0.9998 \\
\hline \multirow{4}{*}{\begin{tabular}{|c|} 
Lead-free \\
soldering \\
material \\
$\mathrm{E}=26.2 \mathrm{GPa}$ \\
$\mathrm{v}=0.3$ \\
\end{tabular}} & \multirow{4}{*}{$\begin{array}{c}22.5 \\
(\mathrm{MPa})\end{array}$} & 0.026 & 1.9017 & 0.9999 \\
\hline & & 0.1 & 2.0229 & 0.9999 \\
\hline & & 0.2 & 2. 1994 & 0.9998 \\
\hline & & 0.3 & 2. 3164 & 0.9999 \\
\hline
\end{tabular}

In the above table, R-squared value is defined as:

$R^{2}=1-\frac{S S E}{S S T}$

where 
$\operatorname{SSE}=\sum\left(Y_{i}-\hat{Y}_{i}\right)^{2}$

and

$S S T=\left(\sum Y_{i}^{2}\right)-\frac{\left(\sum Y_{i}\right)^{2}}{n}$

This R-squared value is a number ranging from 0 to 1 that indicates how well the data points fit the linear equation. An R-squared of ' 1 ' indicates perfect fitting. The closer the R-squared value is to 1 , the better the curve fitting is. In the Table 6.3, it is found the R-squared values for all 21 materials are very close to 1 .

Then the $n$ value vs. $\mathrm{f}(\mathrm{n})$ is plotted to find out the relationship between them.

n vs. $\mathrm{f}(\mathrm{n})$ (slope)

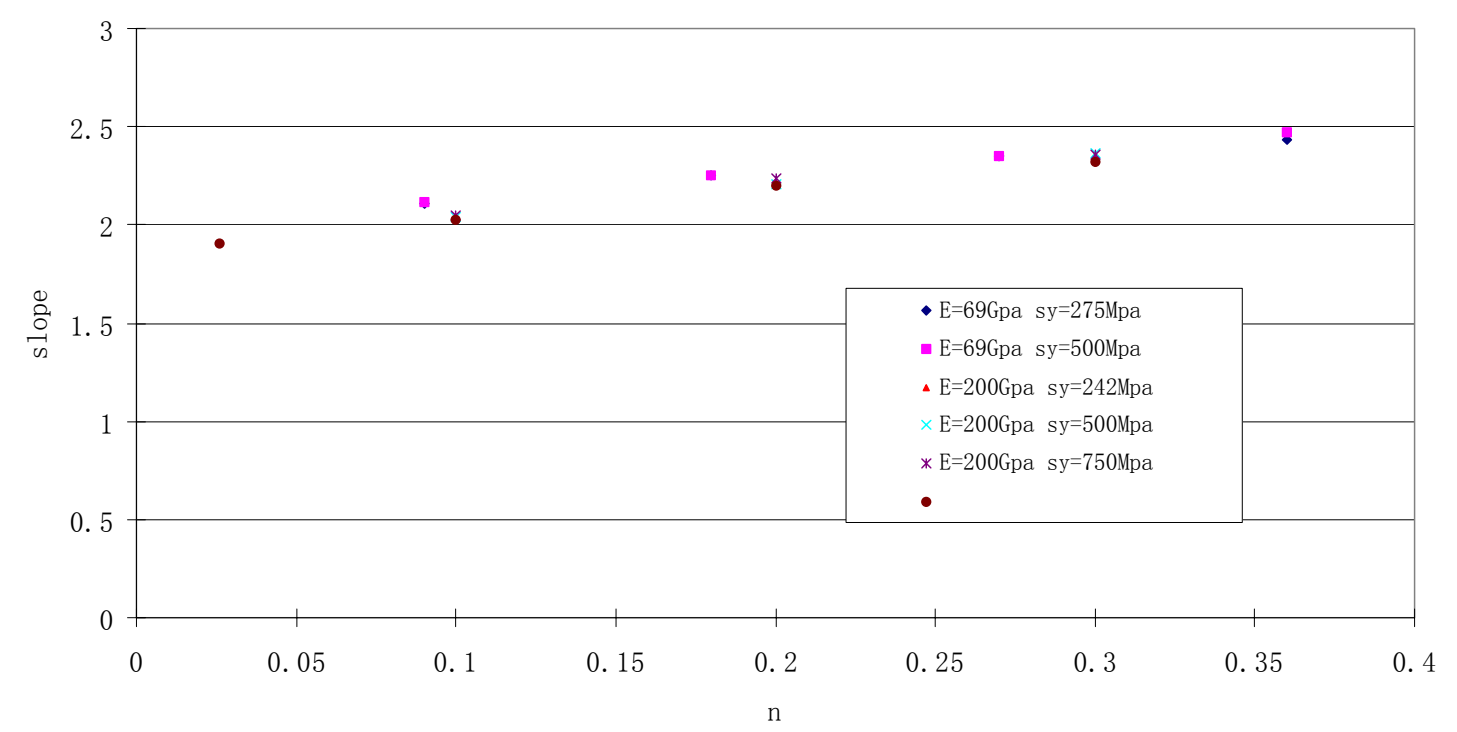

Figure 6.7.2 n vs. $\mathrm{f}(\mathrm{n})$ plot for 21 material models.

It is found from above plot that the slope of $\log (\mathrm{a})$ vs. $\log (\mathrm{P})$ is essentially determined by the strain hardening exponent $n$ value. The variation of Young's modulus and yield stress has insignificant effect on it. Using linear equation to curve fitting the above 21 points, we get the relationship between $\mathrm{n}$ and $\mathrm{f}(\mathrm{n})$, which is:

$f(n)=1.5009 n+1.9187$ 
This modified Meyer's law provides a relatively simple method to estimate material strain hardening behavior based on the experimental measurement of real contact radius a and load $\mathrm{P}$ value. In Equation (6.22), $\mathrm{f}(\mathrm{n})$ represents the slope in the $\log (\mathrm{a})$ vs. $\log (\mathrm{P})$ plot. So $\mathrm{n}$ value is determined once the $\mathrm{f}(\mathrm{n})$ value could be obtained from experiment.

\subsection{Conclusions and discussions}

In a tensile test, the uniaxial deformation is usually contained in the constant volume of the specimen's gage section. Hence, after the completion of elastic/linear loading of a metallic specimen, plastic yielding and work hardening commence and continue until necking occurs. In contrast, in the indentation test the elastic and plastic deformation are not distinctively separated. With increasing indentation penetration depth, an increasing volume of test material is forced to flow under multi axial compression caused by the indenter. Through the surface stress strain analysis, it is found that each point on surface experiences the same stress strain history during the process of loading, which is governed by its uniaxial stress strain curve. The difference between points is the level of deformation due to the different distance from the indenter. In the 3-D indentation, equivalent von Mises stress and total strain are used to relate to uniaxial stress and strain. From the analysis of the finite element simulation results, a series of methodologies are proposed in order to extract bulk material mechanical properties from the indentation test.

\subsubsection{Two methods to get Young's modulus, E value}

Assuming the unloading procedure is pure elastic rebound, two methods could be derived to get the Young's modulus of the indented material: 
1. From the load-depth curve

Using instrumented indentation equipment to record load-depth (P-hs) curve. Then using a linear equation to curve fit the initial part of unloading. The slope of the linear equation is the indentation stiffness: $\frac{d P}{d h_{s}}$. Then the equation $E=\frac{\left(1-v^{2}\right)}{2 \beta} \sqrt{\frac{\pi}{A}} \frac{d P}{d h_{s}}$ is to be employed to calculate Young's modulus.

2. From $\mathrm{W}$ field measurement.

Using an optical method (interferometry) to measure the surface $\mathrm{W}$ field displacement, at both maximum loading and total unloading conditions. Then get the $\mathrm{W}$ field for the unloading process, which is the difference of $\mathrm{W}$ displacement between maximum loading and totally unloading, $W=W_{\text {residual }}-W_{\max \text { Load }}$. Using the equation $\frac{d u_{z}}{d r}=\frac{3 p_{m}\left(1-v^{2}\right)}{4 a E}\left[-2 r \sin ^{-1} \frac{a}{r}+a\left(1-2 \frac{a^{2}}{r^{2}}\right)\left(1-\frac{a^{2}}{r^{2}}\right)^{-1 / 2}+a\left(1-\frac{a^{2}}{r^{2}}\right)^{1 / 2}+a \frac{a^{2}}{r^{2}}\left(1-\frac{a^{2}}{r^{2}}\right)^{-1 / 2}\right]$

to calculate $\mathrm{E}$ value for every location (different $\mathrm{r}$ values) that is in the elastic deformation zone (the elastic-plastic boundary determination will be discussed in 6.8.2). Then, average these values to obtain the Young's modulus of the indented material.

\subsubsection{Yield stress, $\sigma_{\mathrm{y}}$ determination}

In-plane displacement $\mathrm{U}$ and out-of-plane displacement $\mathrm{W}$ fields distribution could be obtained through optical interferometry method. Elastic-plastic boundary under certain loading status corresponds to certain characteristic location of the W-distribution and hence its location can be determined as discussed in Section 6.4. The strain components could be derived from the U-field through the kinematic equations, for both elastic zone and plastic zone. In elastic zone (out of elastic-plastic boundary), Hooke's 
law is employed to calculate the stress components. Then von Mises equivalent stress could be calculated on the elastic-plastic boundary. This stress is the yield stress under uniaxial loading condition.

\subsubsection{Strain hardening exponent determination}

The post-yielding stress strain behavior of the material could be determined using Tabor's relation through cyclic loading on the condition of fully plastic deformation. Using this method, it is not necessary to define any mathematical model to describe the uniaxial stress strain curve. For each loading-unloading cycle, one data point is obtained through Tabor's relation (Equation 6.1). Finally, discrete data points are connected to form a stress strain curve. The simulation results shown in Section 6.2 indicate good agreement with the actual material stress strain curve.

An alternative method, modified Meyer's law, is based on the assumption of a material model; namely elastic power-law plastic stress strain relationship described using Equation (3.3). Then, load (P) vs. real contact radius (a) plots are drawn in

logarithmic scale. A straight line is expected, and the slope can be calculated using linear regression. Then, Equation (6.22) is employed to calculate the $\mathrm{n}$ value, which is the material strain-hardening exponent in Equation (3.3).

\subsubsection{Discussion}

When using the P-hs curve to estimate the Young's modulus of bulk or thin film materials, the estimation accuracy is sensitive to the value of the indentation stiffness $\mathrm{dP} / \mathrm{dhs}$. It is suggested that enough accuracy for the initial unloading load-depth curve must be guaranteed in instrumented indentation tests. 
The post-yielding (plastic) behavior can be obtained through indentation tests either by Tabor's relation or modified Meyer's law. Both of the methods are only valid in the fully plastic region, which requires relatively deeper indentation. How deep the indentation depends on the material yield strength to elastic modulus ratio. The larger the ratio is, the deeper the indentation must be to fulfill the 'fully plastic condition'. The quantitative calculation to determine fully plastic condition is given in Equation (6.2). 


\section{Chapter 7}

\section{Thin film - Substrate System}

\subsection{Introduction}

As discussed in Chapter 2, one of current research potential application areas lies in thin film coating mechanics, which has been widely used in gas turbine, semiconductor, cutting tools, die-casting industries. Here two typical materials, silicon (hard) and aluminum (soft), are selected to form the thin-film substrate system, either of them severed as thin-film or substrate. The material properties used in the calculations are presented in Table 7.1.

Table 7.1 Material properties used for the finite element simulations for thin film substrate system.

\begin{tabular}{|l|c|c|c|c|}
\hline & $\begin{array}{c}\text { Young's Modulus } \\
(\mathrm{GPa})\end{array}$ & Poisson's ratio & $\begin{array}{c}\text { Yield Stress } \\
(\mathrm{MPa})\end{array}$ & $\begin{array}{c}\text { Strain hardening } \\
\text { exponent }\end{array}$ \\
\hline Silicon & 127 & 0.278 & 4410 & 0 \\
\hline Aluminum & 69 & 0.33 & 275 & 0.09 \\
\hline
\end{tabular}

Since the thickness of thin film is usually small (less than $100 \mu \mathrm{m}$ ), a smaller spherical indenter is used, which has diameter of $100 \mu \mathrm{m}$. Maximum depth of indentation is also $1 / 5$ of indenter diameter, which is $20 \mu \mathrm{m}$ in this case.

In finite element modeling, the interface between the bilayer materials is assumed to be perfectly bounded and no fracture would occur during the process of indentation. Similar meshing technique as previous modeling is employed here. The 'stair step' 
pattern of elements is used from the surface elements down throughout the mesh. At the same time, the zoning at the film-substrate interface is kept as dense as those on surface.

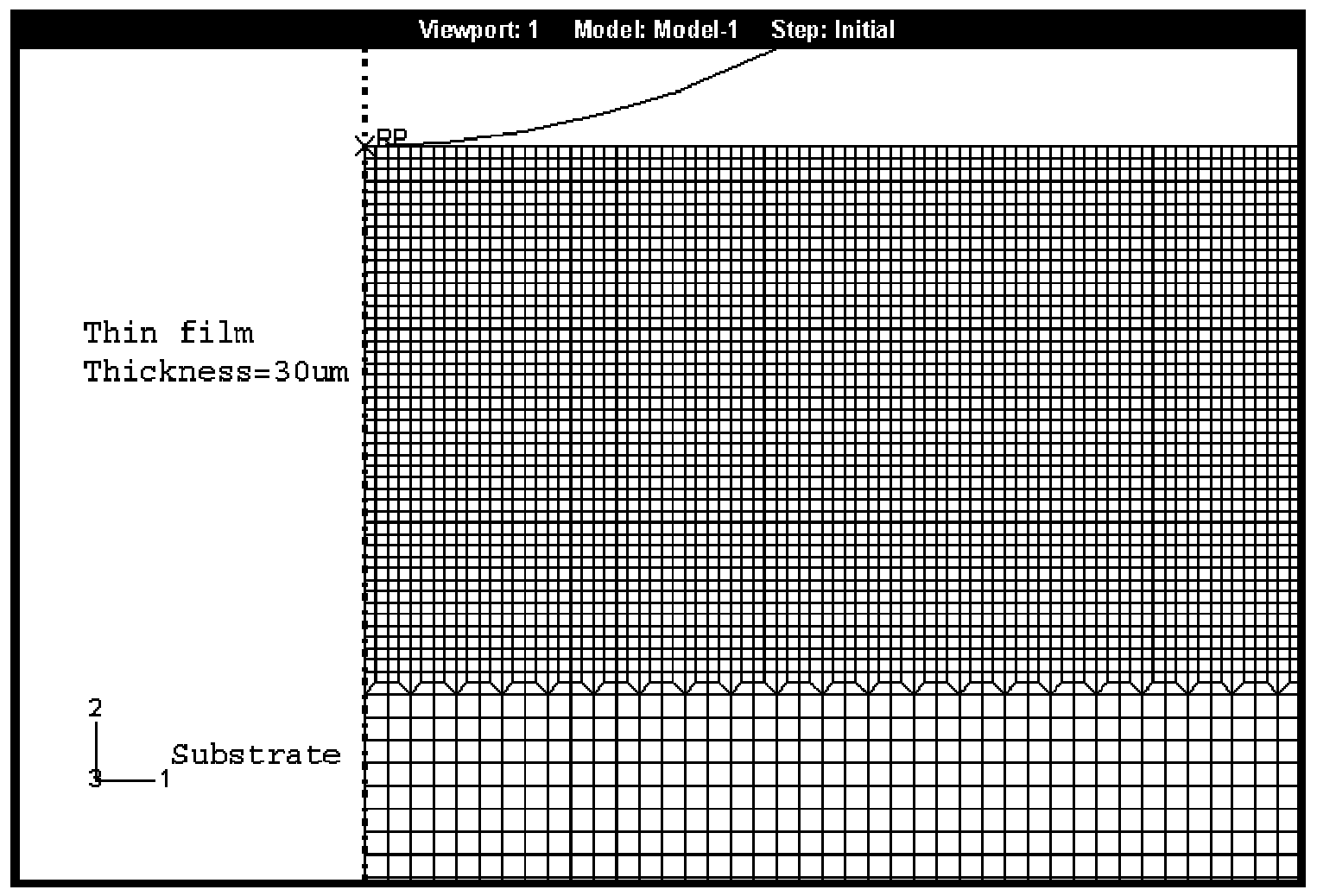

Figure 7.1 Mesh design for thin film substrate system simulations.

\subsection{Soft film on hard substrate and hard film on soft substrate}

At first, simulations of bulk materials are performed respectively for $\mathrm{Al}$ and $\mathrm{Si}$. Then, simulations of two arrangements of thin film and substrate are performed. One arrangement is soft film (Al) on hard substrate ( $\mathrm{Si})$, the other is hard film $(\mathrm{Si})$ on soft substrate (Al). In both models, the thickness of thin film is $30 \mu \mathrm{m}$.

\subsubsection{Load depth curve and Young's modulus calculation}

At first, load depth (P-hs) curves are extracted for all the four cases. 
Load depth (P-hs) curve comparison

(Bulk Si and thin film Si on Al substrate)

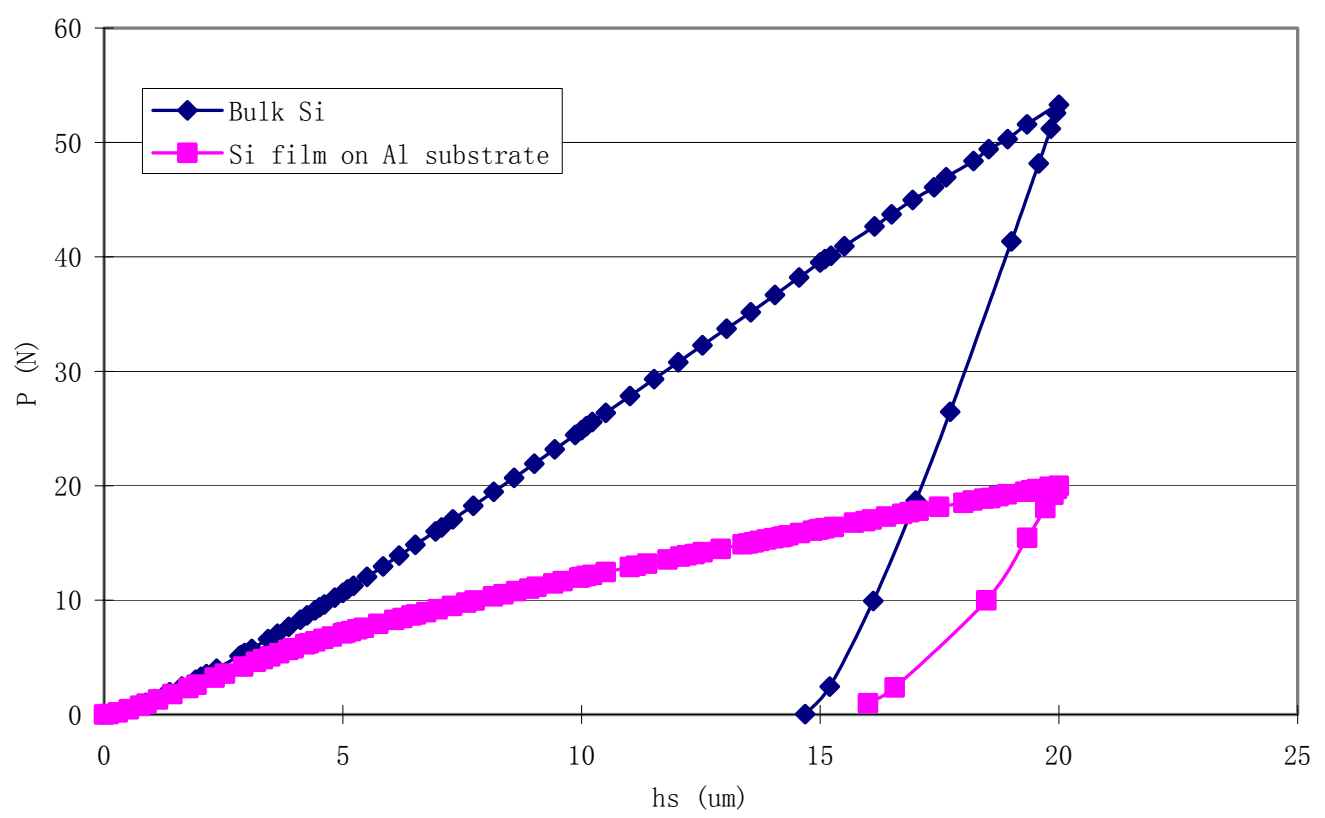

Figure 7.2 (a) Load depth curve for bulk Si and thin film Si on Al substrate.

Load depth (P-hs) curve comparison

(Bulk Al and thin film Al on Si substrate)

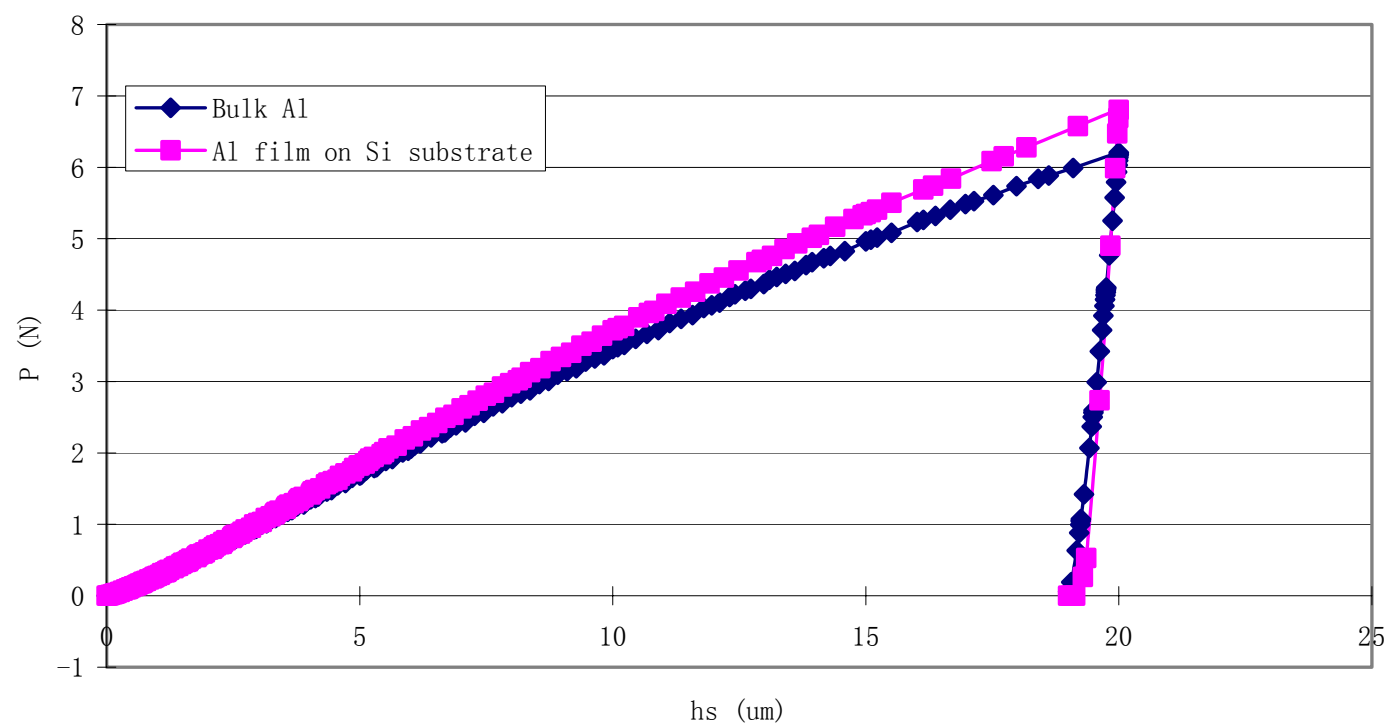

Figure 7.2 (b) Load depth curve for bulk $\mathrm{Al}$ and thin film $\mathrm{Al}$ on Si substrate. 
It is obvious from the loading part of curves that the aluminum film is 'hardened' by the silicon substrate while the silicon film is 'softened' by the aluminum substrate.

For the bulk material, the Young's modulus can be calculated from initial unloading slope (indentation stiffness), which has been described in detail in Chapter 2 and Section 6.3.

Table 7.2 Using indentation stiffness to calculate elastic modulus for bulk materials.

\begin{tabular}{|l|c|c|c|c|c|c|c|}
\hline & $\begin{array}{c}\text { Input Young's } \\
\text { Modulus (GPa) }\end{array}$ & $\begin{array}{c}\mathrm{dP} / \mathrm{dhs} \\
(\mathrm{N} / \mu \mathrm{m})\end{array}$ & $\mathrm{a}(\mu \mathrm{m})$ & $\begin{array}{c}\mathrm{A} \\
\left(\mu \mathrm{m}^{2}\right)\end{array}$ & $\beta$ & $\mathrm{E}_{\text {cal }}(\mathrm{GPa})$ & Error (\%) \\
\hline $\mathrm{Si}$ & 127 & 11.993 & 38.312 & 4611.26 & 1.1 & 131.29 & 3.38 \\
\hline $\mathrm{Al}$ & 69 & 7.567 & 42.408 & 5649.96 & 1.09 & 72.8 & 5.5 \\
\hline
\end{tabular}

As claimed previously in Section 6.3, this method to calculate bulk materials' elastic modulus from indentation tests is reliable and accurate. The error is limited in $6 \%$.

In the literature review of Chapter 2.6, it has been described that R.B. King [5] has derived equations to calculate thin film elastic property from unloading indentation stiffness. Based on current finite element simulation results, King's Equation (2.27) is employed to calculate thin film elastic modulus. Results are shown in Table 7.3, in which $\alpha$ parameter is determined from $\sqrt{A} / t$ by curve shown in Figure 7.3. 

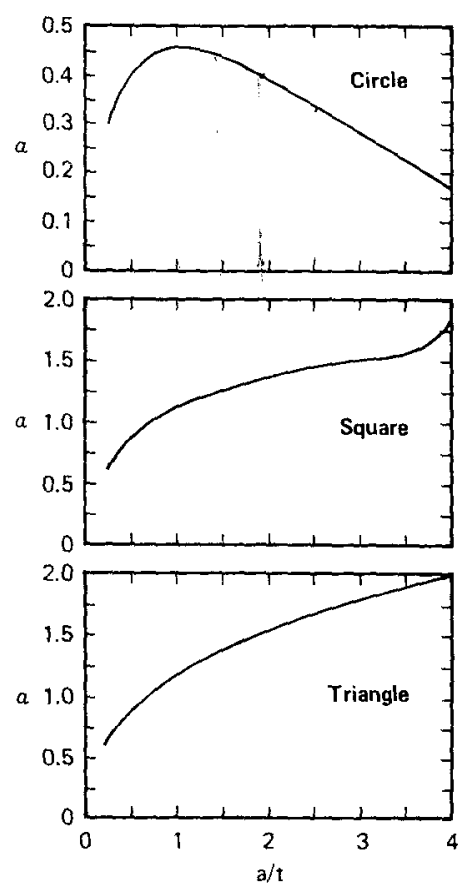

Figure 7.3 Parameter $\alpha$ as a function of normalized punch size.

(Courtesy of R.B. King, [5])

Table 7.3 Using indentation stiffness to calculate elastic modulus for thin film materials.

\begin{tabular}{|c|c|c|c|c|c|c|c|}
\hline $\begin{array}{c}\text { Input Young's } \\
\text { Modulus }(\mathrm{GPa})\end{array}$ & $\begin{array}{c}\mathrm{dP} / \mathrm{dhs} \\
(\mathrm{N} / \mu \mathrm{m})\end{array}$ & $\mathrm{a}(\mu \mathrm{m})$ & $\begin{array}{c}\mathrm{A} \\
\left(\mu \mathrm{m}^{2}\right)\end{array}$ & $\sqrt{A} / t$ & $\alpha$ & $\mathrm{E}_{\text {cal }}(\mathrm{GPa})$ & $\begin{array}{c}\text { Error } \\
(\%)\end{array}$ \\
\hline $\begin{array}{c}\text { Si film on Al sub } \\
\text { Si: } 127 \\
\text { Al: } 69 \\
\mathrm{t}=30 \mu \mathrm{m}\end{array}$ & 6.87 & 32.649 & 3348.8 & 1.93 & 0.37 & 138.21 & 8.83 \\
\hline $\begin{array}{c}\text { Al film on Si sub } \\
\text { Al: } 69 \\
\text { Si: } 127 \\
\mathrm{t}=30 \mu \mathrm{m}\end{array}$ & 11.3 & 43.287 & 5886.6 & 2.56 & 0.3 & 79.98 & 15.92 \\
\hline
\end{tabular}

The error percentage obtained from King's formula is greater than that from method to evaluate elastic modulus of bulk materials. However, the level of agreement with the theory presented by King and current FEM simulation results is encouraging. It is also found the calculation results are sensitive to the value of $\mathrm{dP} / \mathrm{dhs}$ and $\alpha$. Much 
more accurate results could be expected by modifying $\alpha$ vs. $\sqrt{A} / t$ curve presented by King.

\subsubsection{Plastic zone}

We first refer to Figures 7.4 and 7.5, which display the computed plastic zones (for $20 \mu \mathrm{m}$ indentation depth) for bulk $\mathrm{Al}$ and $\mathrm{Si}$ and the two types of film, respectively.

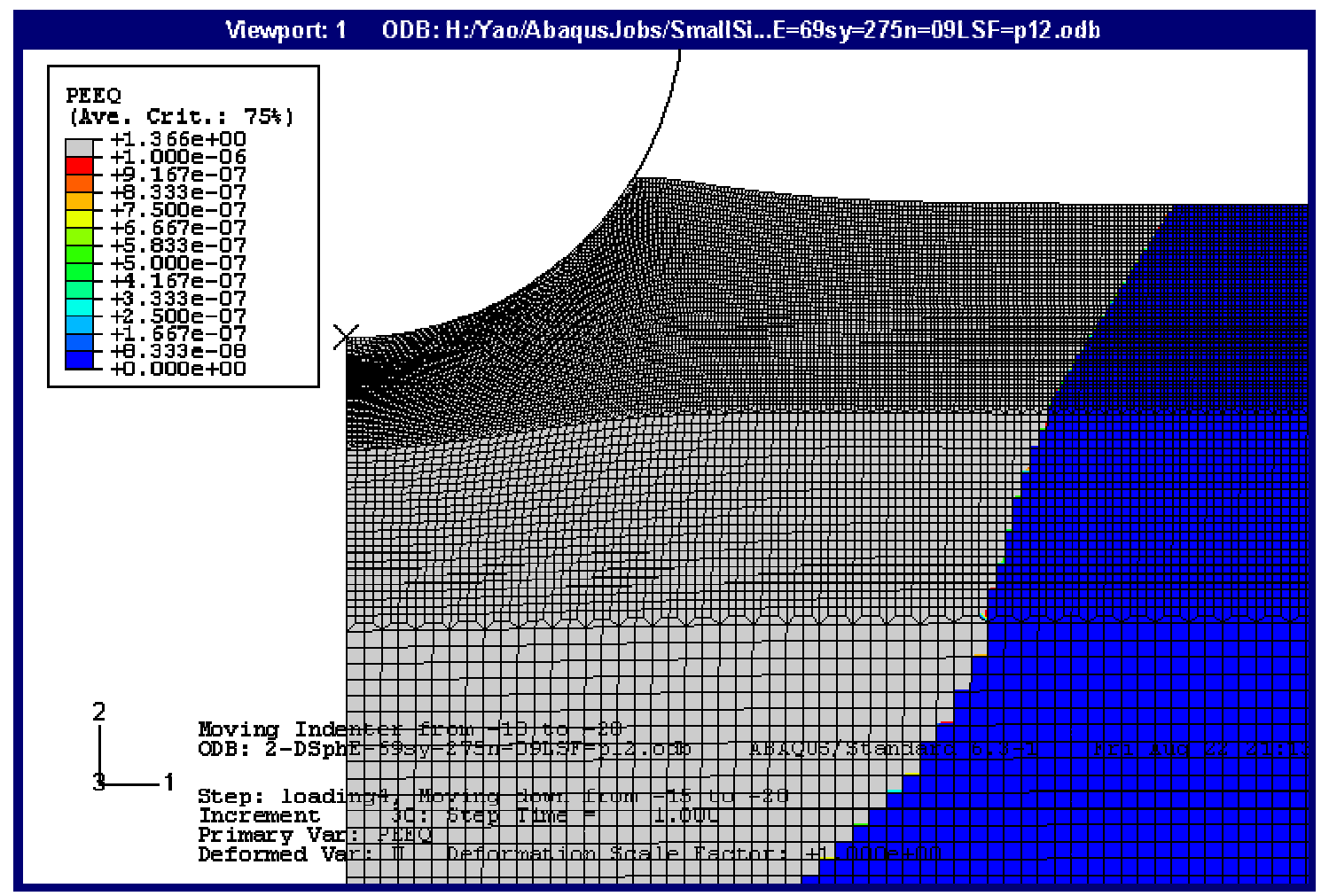

Figure 7.4 (a) Computed plastic regions for bulk aluminum. 


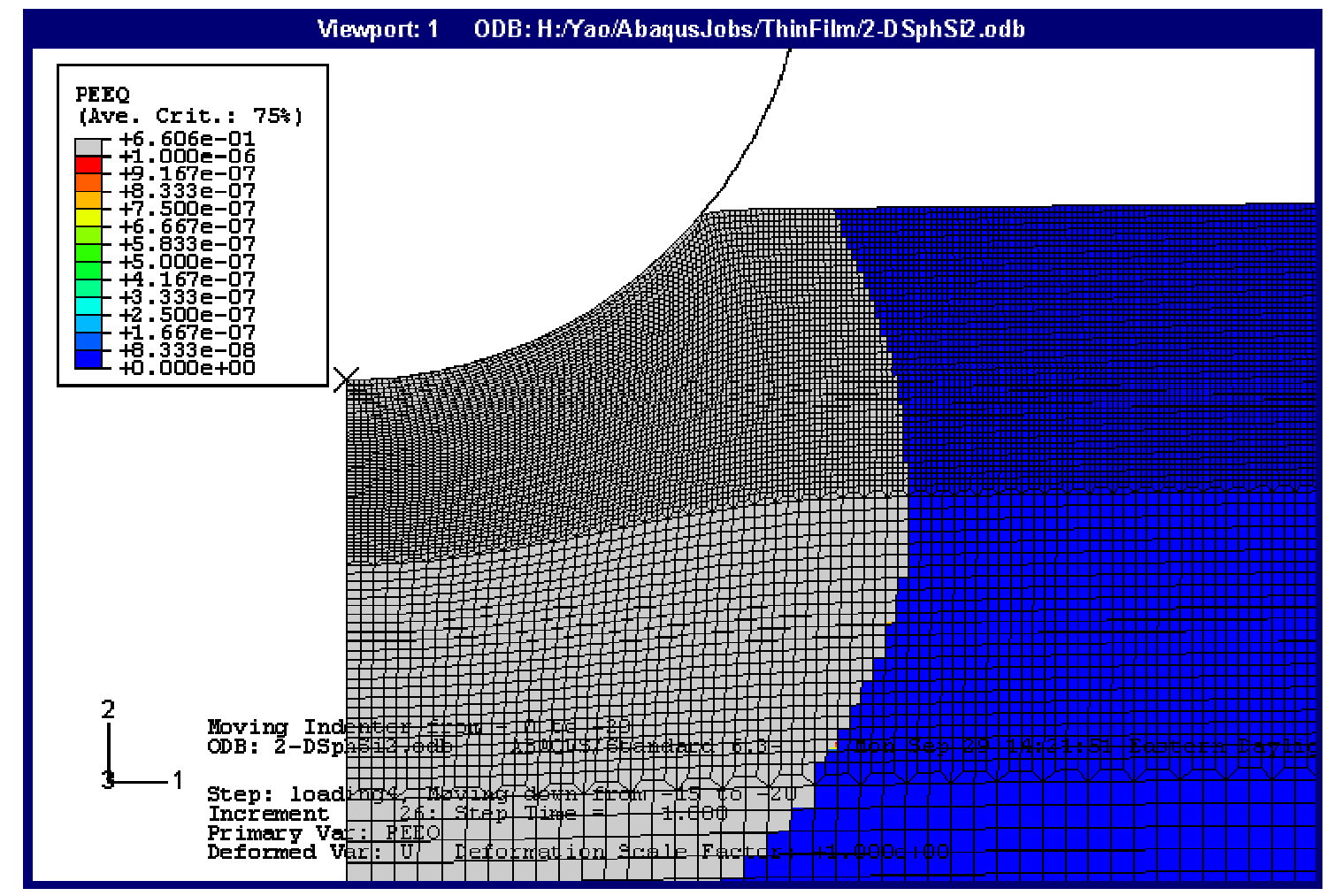

Figure 7.4 (b) Computed plastic regions for bulk silicon.

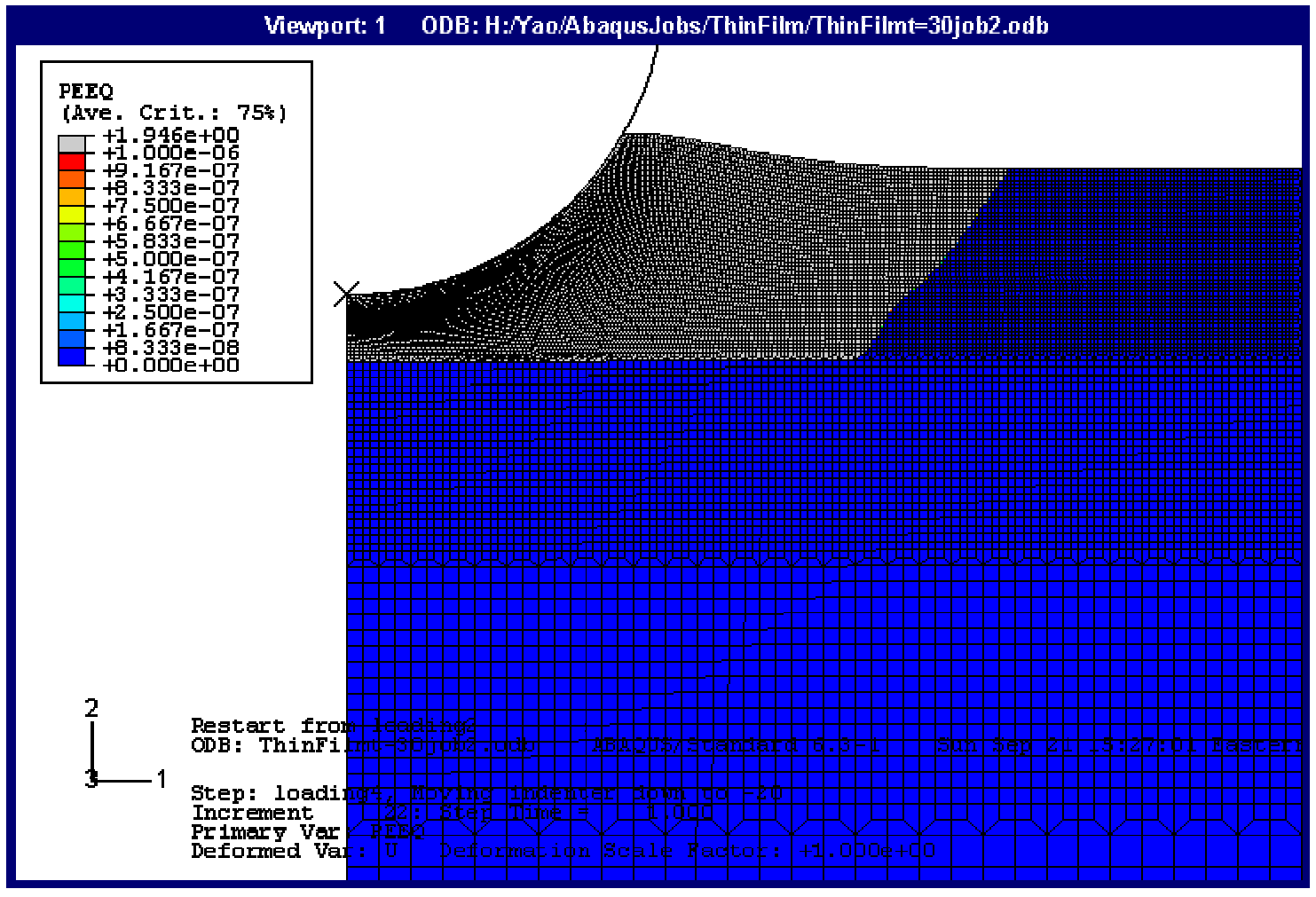

Figure 7.5 (a) Computed plastic zone of soft film (Al) on hard substrate (Si). 


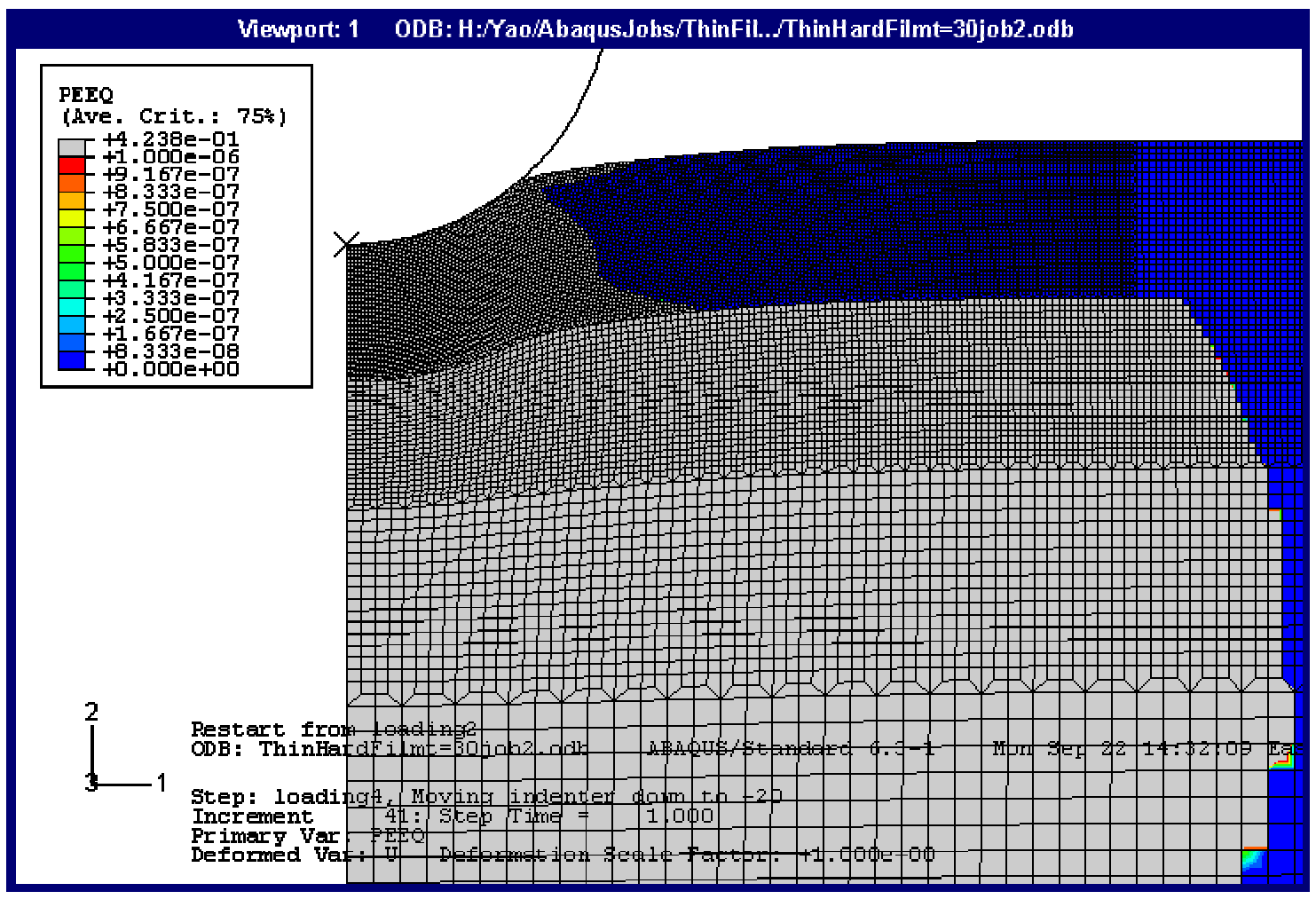

Figure 7.5 (b) Computed plastic zone of hard film (Si) on soft substrate (Al).

As can be noted from Figures 7.4, the softer aluminum displays a much more extensive plastic zone than the relatively harder silicon under the same indentation depth. Figures 7.5 indicate that for the case of softer film on a harder substrate, the plasticity is confined almost exclusively to the film. On the other hand, the opposite arrangement leads to response in which the plasticity is induced mostly in the substrate, with only a small plastic region in the film directly under the indenter.

These observations of plastic zone are in consistent with the conclusion made by T.A. Laursen et al, [52], who pointed out 'as a consequence of these facts, it is clear that any idealization that might be used to provide a simpler analysis of film response must take into account the fact that soft-on-hard and hard-on-soft film systems respond in very different ways'.

\subsubsection{Tabor's relation}


As described in Section 6.2, the Tabor's relation from indentation is in good agreement with uniaxial stress strain curve for bulk materials. Then what is the substrate effect on the thin film material Tabor's relationship?

It is indicated in Figure 7.6 (a) that the Tabor's relation for thin film aluminum is in a worse agreement with the true stress-true strain curve compared with bulk aluminum. For the soft film on hard substrate case, the Tabor's relation gives higher stress data. The effect of substrate cannot be ignored especially when the indentation depth is comparable with the coating thickness. The hard substrate effect will give a higher hardness (mean pressure $\mathrm{p}_{\mathrm{m}}$ ) value, which explains why the thin film stress given by Tabor's relation is higher than bulk stress.

Tabor's relation

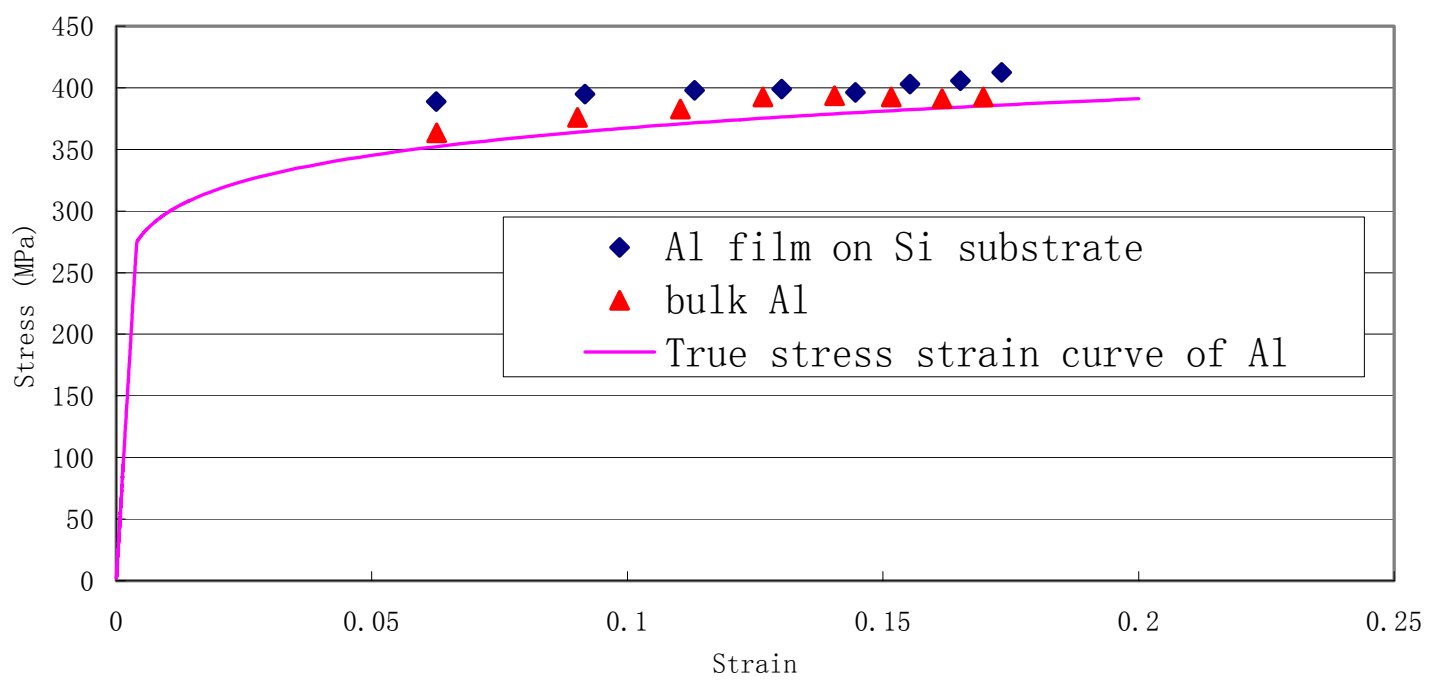

Figure 7.6 (a) Tabor's relation comparison for thin film Al and bulk Al. 
Tabor's relation

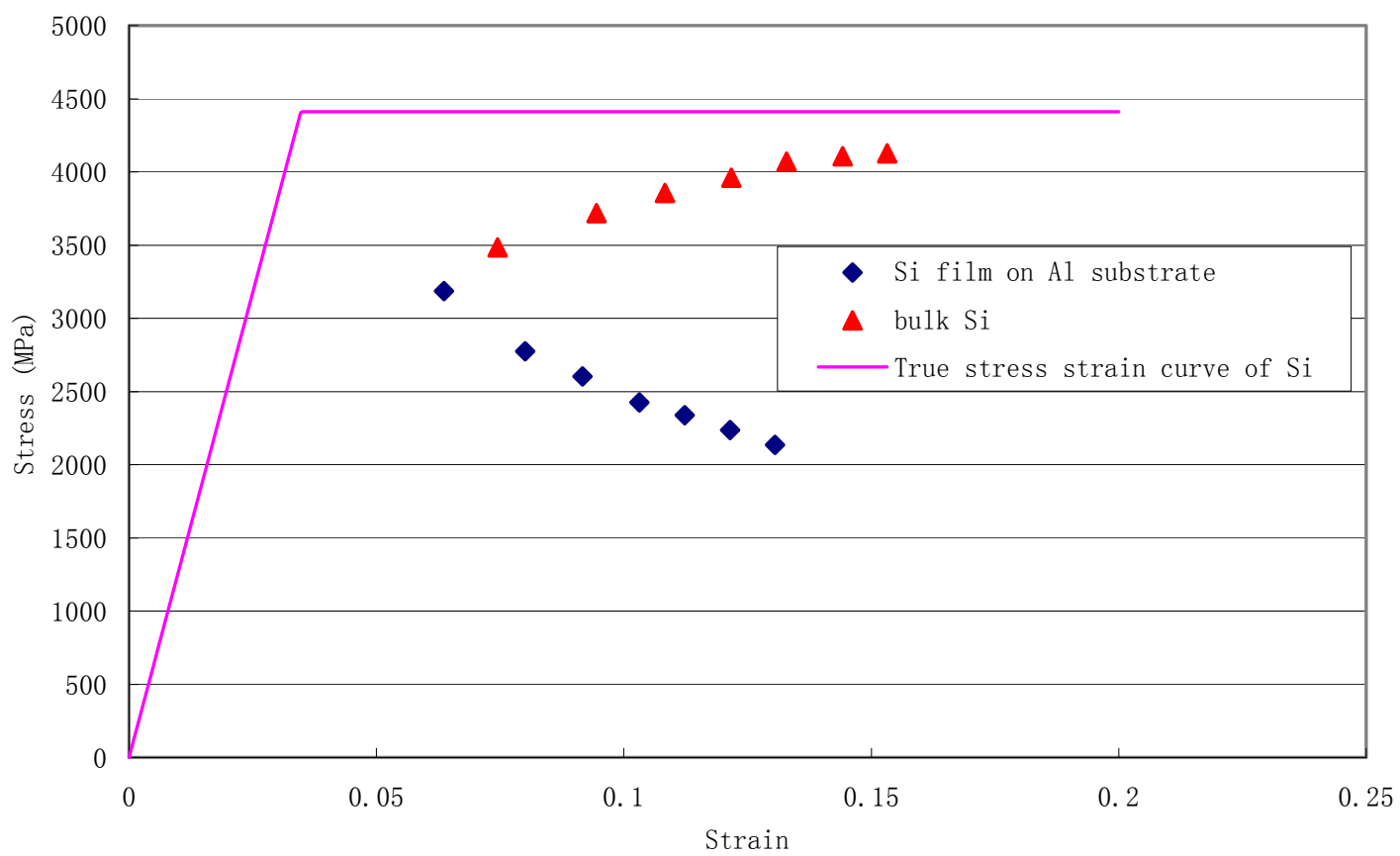

Figure 7.6 (b) Tabor's relation comparison for thin film Si and bulk Si.

Direct observation of Figure 7.6 (b) reminds us that the Tabor's relation is only applicable in fully plastic region in indentation test. The agreement between the Tabor's relation and original stress strain curve for bulk $\mathrm{Si}$ is poor. That can be attributed to the relatively large elastic portion $\left(\sigma_{y} / E\right)$ for this material model. Plug the E and $\sigma_{\mathrm{y}}$ of Si into Equation (6.2) and we get:

$\varepsilon_{\min }=0.2 \sin \left[\tan ^{-1}\left(30 \varepsilon_{e}\right)\right]=0.144$

which indicates that even deeper indentation is needed to get fully plastic condition. That also explains why the indentation stress strain data is getting close to the input for deeper indentation (large strain) rather than shallow indentation (small strain). 
For the thin film silicon on aluminum substrate, Tabor relation result is getting worse with the increasing of indentation depth. The predicted stress using Tabor's relation is much smaller than the actual stress. Since the influence of the soft substrate $\mathrm{Al}$, the deeper the indentation goes, the more discrepancy between Tabor's relation and true stress strain curve is found. This result is the expected character: the soft substrate (Al) makes the Meyer's hardness, which is mean pressure $\left(\mathrm{p}_{\mathrm{m}}\right)$, decrease with the increase of indentation depth.

It is concluded that the Tabor's relation cannot be employed to get true stress strain curve for thin film materials. The effect of substrate cannot be neglected especially when the indentation depth is comparable with the film thickness. For soft film on hard substrate, the stress obtained from Tabor's relation is higher than that in true stress strain curve, while for hard film on soft substrate, the stress from Tabor's relation is smaller than that in true stress strain curve of film material.

\subsubsection{Surface deformation}

Out-of-plane displacement $\mathrm{W}$ fields are extracted out from simulation results for all 4 cases and comparisons are made.

It is obvious in Figure 7.7 (a) that there is much piling up in aluminum thin film on silicon substrate than in bulk aluminum. Also the elastic-plastic boundary is much closer to the indenter for $\mathrm{Al}$ thin film case, which is also corresponding to the lowest point on the $\mathrm{W}$ field contour. 
W field distribution at maximum loading depth (hs=20um)

(Comparison between bulk Al and thin film Al on Si substrate)

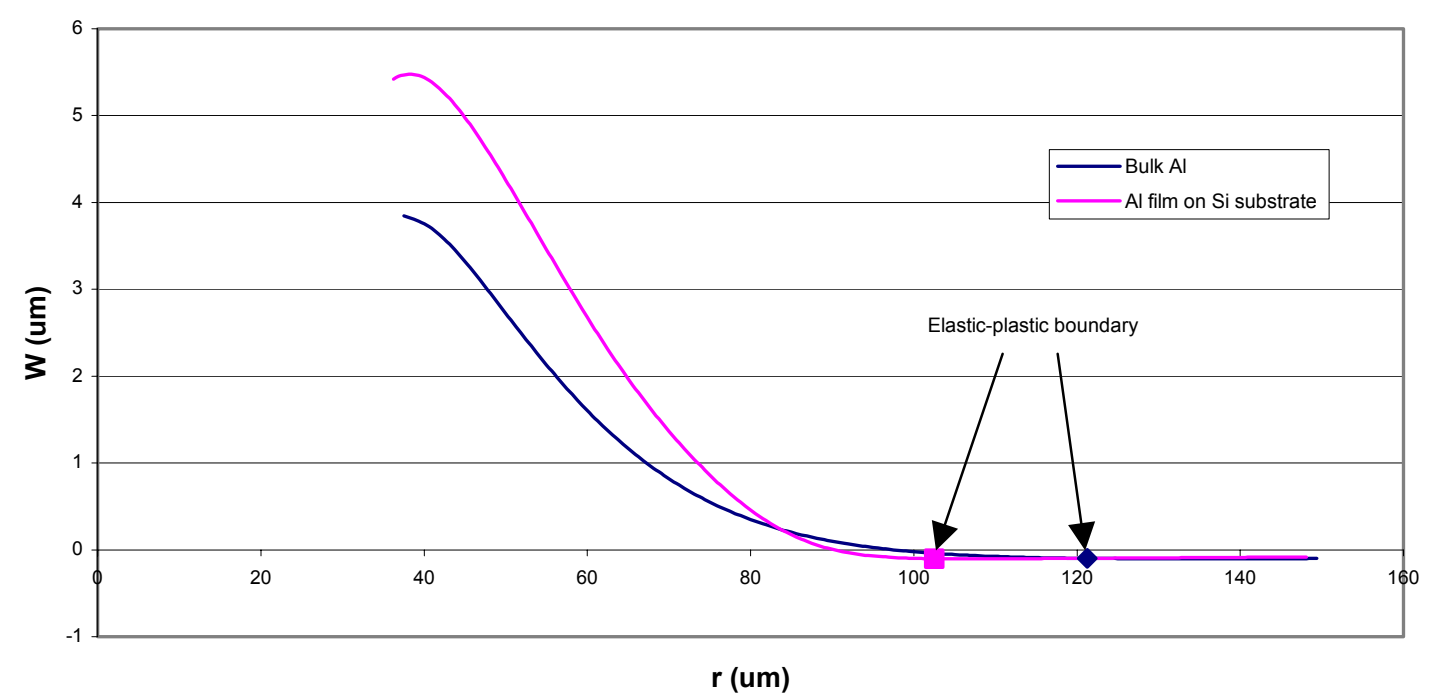

Figure 7.7 (a) W field distribution: comparison between bulk Al and thin film Al.

W field distribution at maximum loading depth (hs=20um) (Comparison between bulk $\mathrm{Si}$ and thin film $\mathrm{Si}$ on Al substrate)

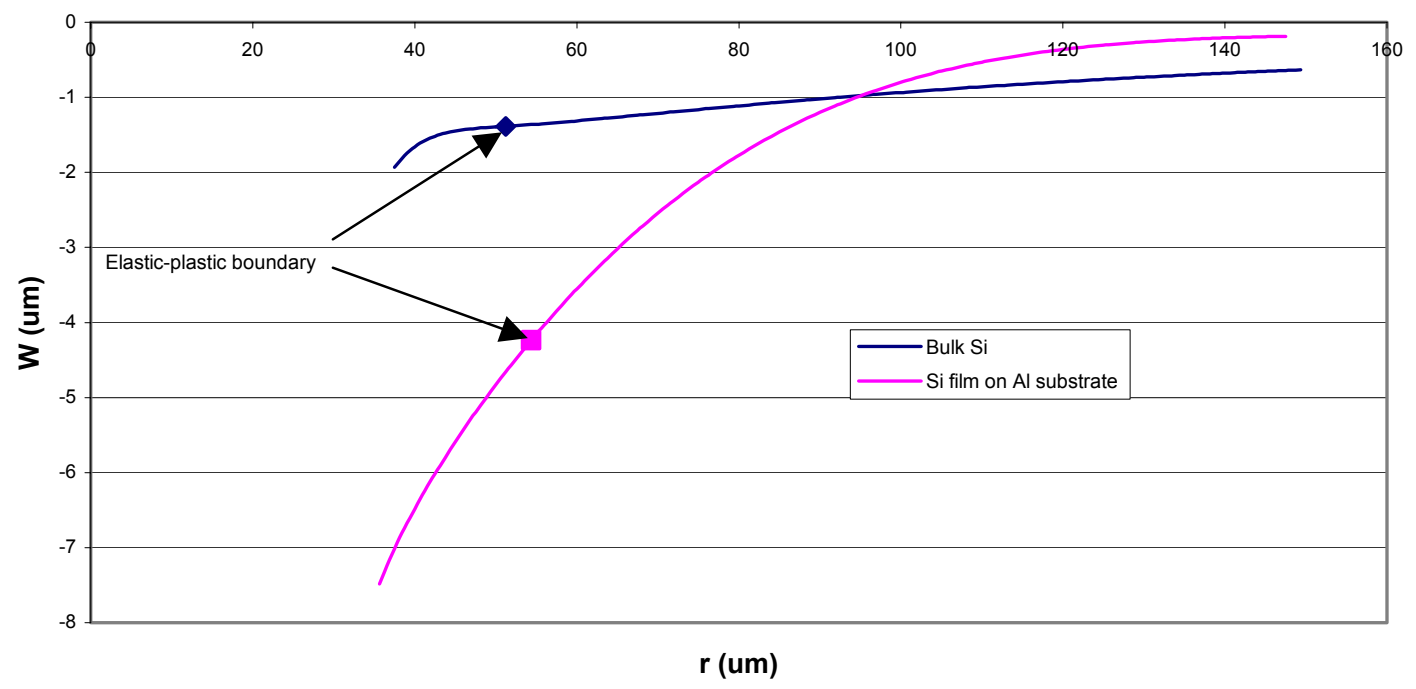

Figure 7.7 (b) W field distribution: comparison between bulk Si and thin film Si.

Observation of blue curve in Figure 7.7 (b) indicates that the conclusions on the relationship between strain hardening exponent $\mathrm{n}$ and surface deformation mode (put forward by Alcala et al [18]) is not necessarily to be true for all materials. For bulk 
silicon, the material property is given as $n=0$ (elastic perfectly plastic). According to [18], the piling up should be predominant surface deformation mode. However, it is obvious in Figure 7.7 (b) that bulk silicon surface is sinking in. When the silicon is made as thin film on aluminum substrate, the sinking in effect is getting more obvious. The location of elastic-plastic boundary doesn't change much compared with bulk silicon.

\subsection{Thin film thickness effect}

To study the thin film thickness effect, two finite element models are established in which the thickness of aluminum film is $100 \mu \mathrm{m}$ on silicon substrate and $100 \mu \mathrm{m}$ thickness Si film on Al substrate. All the other conditions are not changed. Then the simulation results (load-depth curve and surface displacement $\mathrm{W}$ field) are compared with the bulk aluminum and $30 \mu \mathrm{m}$ thickness film.

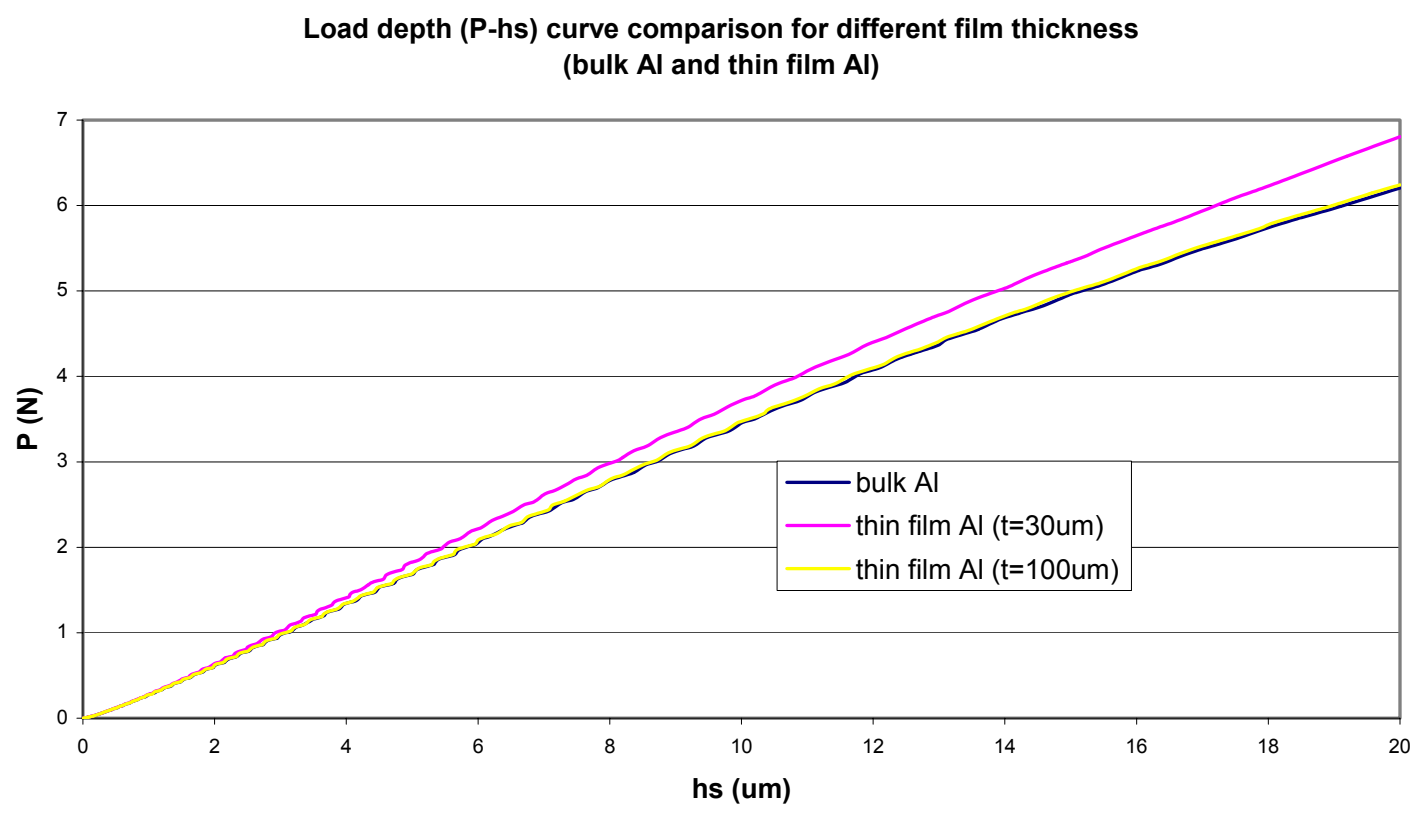

Figure 7.8 (a) Load depth curve for different thickness of aluminum thin film. 
W field distribution at maximum loading depth (hs=20um)

(Comparison between bulk Al and thin film Al on Si substrate for different film thickness)

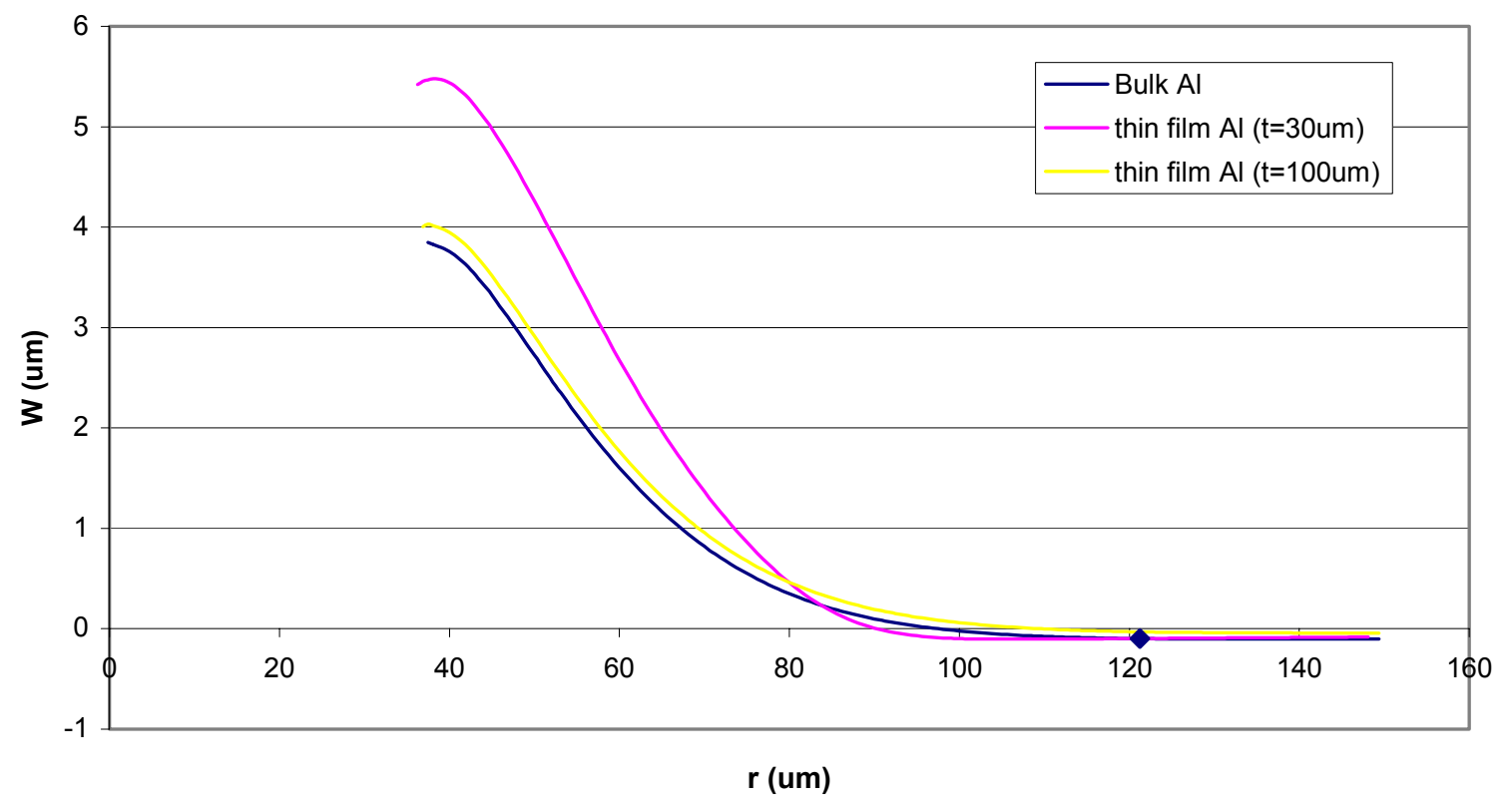

Figure 7.8 (b) W field distribution: comparison for different film thickness.

Load depth (P-hs) curve comparison for different film thickness (bulk Si and thin film Si)

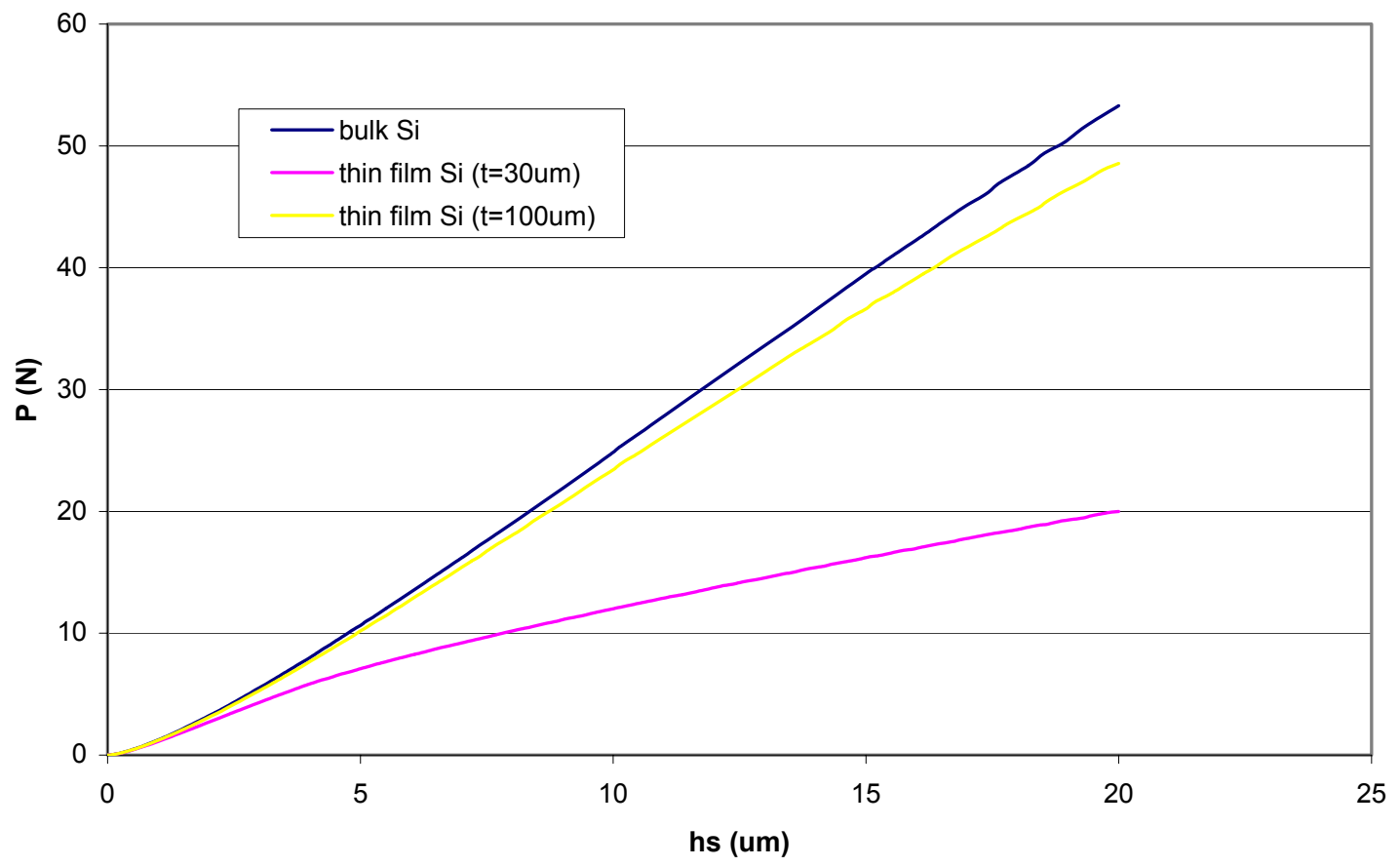

Figure 7.9 Load depth curve for different thickness of silicon thin film. 
It is found that the load depth curve of the $100 \mu \mathrm{m}$ thick $\mathrm{Al}$ thin film is almost overlapped over that of the bulk aluminum, while the $30 \mu \mathrm{m}$ thickness has much more deviation from the bulk curve, which indicate the thicker film will diminish the substrate effect on the indentation data. However, for bulk Si and thin film Si observation in Figure 7.9, the thin film load depth curve is obviously deviated from that of bulk $\mathrm{Si}$, even for $100 \mu \mathrm{m}$ thickness film. It indicates that for the hard film on soft substrate case, the substrate effect is much more pronounced than the soft film on hard substrate case when the film thickness is the same.

It is commonly agreed that when the indentation depth is less than $10 \%$ to $20 \%$ of the film thickness the substrate effect is negligible. From our study, this conclusion could only apply for soft film on hard substrate case. For hard film on soft substrate, the ratio of indentation depth to film thickness should be even smaller to eliminate the effect of substrate. 


\section{Chapter 8}

\section{Conclusions and Discussions}

It is shown that finite element analysis may be effectively used to aid in characterization of the indentation process in both bulk material and thin film - substrate system. Numerical simulations give information difficult to obtain experimentally (area of contact, yielded zone, stress, strain, displacement, etc.), and thus provide a tool that enables better understanding of the mechanics involved in indentation, which provides significant instructions for later experimental work. Finite element simulations of spherical indentation on both bulk and thin film materials are performed. Various influential effects are considered and discussed during the FE modeling process. Both Hertz elastic solution and real optical measurement of surface displacement fields are employed to verify the finite element simulation results. Comprehensive analysis of 21 material models' simulation results, focused on surface displacement fields in addition to the Tabor's relation and load depth curve, are performed to develop a series of methodology to extract material mechanical properties (tensile stress strain curve).

For bulk materials, it is relatively easier to estimate mechanical properties from indentation test. Details have been concluded in Chapter 6. Thin film - substrate system indentation is much more complicated, due to the influence of substrate. At first the King's formula to estimate thin film elastic modulus from indentation stiffness is studied and verified. Then Tabor's relation and surface deformation are studied for thin film substrate indentation. The effect of substrate is becoming more and more obvious when the indentation depth is comparable with thin film thickness. Because of the different shape of plastic zone between hard film on soft substrate and soft film on hard substrate, 
it is necessary to take into account the fact that soft-on-hard and hard-on-soft film systems respond in very different ways. Then by simulating different thickness of thin film, it is found that for the hard film on soft substrate case, the substrate effect is much more pronounced than the soft film on hard substrate case for the same film thickness.

The straightest and simplest way to obtain thin film mechanical properties is to eliminate the substrate effect by minimizing the size of the indenter and limiting indentation depth within certain amount of thin film thickness. For hard film on soft substrate system, the limit should be much smaller than that for soft film on hard substrate case.

It is suggested that future research should focus on the experimental realization of proposed methodology for bulk materials. Much more detailed research work should be performed for thin film - substrate system indentation. 


\section{Bibliography:}

[1] Ian N. Sneddon, 'The Relation between Load and Penetration in The Axisymmetric Boussinesq Problem For A Punch Of Arbitrary Profile', Int. J. Engng Sci, Vol. 3, pp. 4757, 1965

[2] K. L. Johnson, 'The Correlation of Indentation Experiments', J. Mech. Phys. Solids, Vol. 18, pp. 115-126, 1970

[3] K. L. Johnson, 'Contact Mechanics', Cambridge University Press, Cambridge, UK, 1985

[4] R. Hill, 'The Mathematical Theory of Plasticity', Clarendon Press, Oxford, 1950

[5] R. B. King, 'Elastic Analysis of Some Punch Problems for a Layered Medium', Int. J. Solids Structures, Vol. 23, No. 12, pp. 1657-1664, 1987

[6] J. L. Loubet, J. M. Georges, J. M. Marchesini, and G. Meille, 'Vicker’s Indentation Curves of Magnesium Oxide (MgO)', J. Tribology, 106, 43, 1984

[7] A.C. Fischer-Cripps, 'Use of Combined Elastic Modulus in the Analysis of DepthSensing Indentation Data', Journal of Materials Research, Vol 16, pp. 3050-3052, 2001

[8] M. F. Doerner, W. D. Nix, 'A Method For Interpreting the Data From Depth-Sensing Indentation Instruments', J. Mater. Research, 4, 601, 1986

[9] G.M. Pharr, W.C. Oliver, and F.R. Brotzen, 'On the Generality of the Relationship Among Contact Stiffness, Contact Area, and Elastic Modulus During Indentation', Journal of Materials Research, Vol 7, pp. 613-617, 1992

[10] A. K. Bhattacharya, W. D. Nix, 'Finite Element Simulation of Indentation Experiments', Int. J. Solids Structures, Vol. 24, No.9, pp. 881-891, 1988 
[11] A. K. Bhattacharya, W. D. Nix, 'Analysis of Elastic and Plastic Deformation Associated with Indentation Testing of Thin Films on Substrates', Int. J. Solids Structures, Vol. 24, No. 12, pp. 1287-1298, 1988

[12] W. C. Oliver, G. M. Pharr, 'An Improved Technique for Determining Hardness and Elastic Modulus Using Load and Displacement Sensing Indentation Experiments', J. Mater. Res, Vol. 7, No. 6, pp. 1564-1583, 1992

[13] M. Dao, N. Chollacoop, K. J. Van Vliet, T. A. Venkatesh and S. Suresh, 'Computational Modeling of the Forward and Reverse Problems in Instrumented Sharp Indentation', Acta Mater, Vol. 49, pp 3899-3918, 2001

[14] F. M. Haggag, H. Wong, D. J. Alexander, R. K. Nanstad, 'The Use of Field Indentation Microprobe in Measuring Mechanical Properties of Welds', Recent Trends in Welding Science and Technology, Proceedings of the $2^{\text {nd }}$ International Conference on Trends in Welding Research, Gatlinburg, Tennessee, pp. 843-849, 1989

[15] K. L. Murty, M. D. Mathew, P. Q. Miraglia, V. N. Shah and F. M. Haggag, 'Nondestructive Evaluation of Deformation and Fracture Properties of Materials Using StressStrain Microprobe', Non-destructive Characterization of Materials in Aging Systems, Materials Research Society, Penn, 1998

[16] A. Bolshakov, G. M. Pharr, 'Influence of Pileup on the Measurement of Mechanical Properties by Load and Depth Sensing Indentation Techniques', J. Mater. Res, Vol. 13, No. 4, pp. 1049-1058, 1998

[17] Yang-Tse Cheng, Che-Min Cheng, 'Can Stress-Strain Relationships be obtained from indentation Curves Using Conical and Pyramidal Indenters?', Journal of Materials Research, Vol. 14, pp. 3493-3496, 1999 
[18] J. Alcala, A.C. Barone and M. Anglada, 'The Influence of Plastic Hardening on Surface Deformation Modes Around Vickers and Spherical Indents', Acta Materialia, Vol. 48, pp. 3451-3464, 2000

[19] A.E. Giannakopoulos and S. Suresh, 'Determination of Elastoplastic Properties by Instrumented Sharp Indentation', Scripta Materialia, Vol. 40, pp. 1191-1198, 1999 [20] M. Mata, M. Anglada and J. Alcala, 'Contact Deformation Regimes Around Sharp Indentations and the Concept of the Characteristic Strain', Journal of Materials Research, Vol. 17, pp. 964-976, 2002

[21] J. Alcala, A.E. Giannakopoulos, and S. Suresh, 'Continuous Measurements of Loadpenetration Curves with Spherical Microindenters and the estimation of Mechanical Properties', Journal of Materials Research, Vol. 13, pp. 1390-1400, 1998

[22] Xi Chen and Joost J. Vlassak, 'Numerical Study on the Measurement of Thin Film Mechanical Properties by Means of Nanoindentation', Journal of Materials Research, Vol. 16, pp. 2974-2982, 2001

[23] Standard Test Method for Vickers Hardness of Metallic Materials, American Society for Testing and Materials, West Conshohocken, Pa, 1987

[24] Kurt E. Peterson and C.R. Guarnieri, 'Young's Modulus Measurements of Thin Films Using Micromechanics', Journal of Applied Physics, Vol.50, pp. 6761-6766, 1979 [25] N. A. Fleck and J.W. Hutchinson, 'A Phenomenological Theory for Strain Gradient Effects in Plasticity', Journal of Mechanics and Physics of Solids, Vol. 41, pp. 18251857,1993 
[26] H. Gao, Y. Huang, W.D. Nix and J. W. Hutchinson, 'Mechanism-based Strain Gradient Plasticity - I. Theory', Journal of the Mechanics and Physics of Solids, Vol. 47, pp. 1239-1263, 1999

[27] Y. Huang, H. Gao and J. W. Hutchinson, 'Mechanism-based Strain Gradient Plasticity. II. - Analysis', Journal of the Mechanics and Physics of Solids, Vol. 48, pp. $99-128,2000$

[28] Y. Huang, Z. Xue, H. Gao, W.D. Nix and Z.C. Xia, 'A Study of Micro-indentation Hardness Tests by Mechanism-based Strain Gradient Plasticity, Journal of Materials Research, Vol. 15, pp. 1786-1796, 2000

[29] M. F. Doerner, D. S. Gardner and W. D. Nix, 'Plastic Properties of Thin Films on Substrates as Measured by Submicron Indentation Hardness and Substrate Curvature Techniques', Journal of Materials Research, Vol. 1, pp. 845-851, 1987

[30] H. Gao, C-H. Chiu and J. Lee, 'Elastic contact versus indentation modeling of multilayered materials', International Journal of Solids and Structures, Vol. 29, pp. 2471-2492, 1992

[31] ABAQUS Version 6.3 User's Manual

[32] J.C. Hay, A. Bolshakov, G.M. Pharr, 'A critical examination of the fundamental relations used in the analysis of nanoindentation data', Journal of Materials Research, Vol. 14, pp. 2296-2305, 1999

[33] J.L. Hay, P.J. Wolff, 'Small Correction Required When Applying the Hertzian Contact Model to Instrumented Indentation Data', Journal of Materials Research, Vol. 16, pp. 1280-1286, 2001

[34] www.carbidedepot.com 
[35] A.F. Bower and B. Storakers, J. Mech. Phys. Solids 43, 1303 (1995)

[36] K. Komvopoulos, J. Tribol. 110, 477 (1998)

[37] S. D. Mesarovic and N. A. Fleck, Proc. R. Soc. A 455, 2707 (1999)

[38] Aditad Vasinonta, Jack L. Beuth, 'Measurement of interfacial toughness in thermal barrier coating systems by indentation', Engineering Fracture Mechanics, 68, 843-860 $(2001)$

[39] D. Tabor, Hardness of Metals, (Charendon Press, Oxford, United Kingdom, 1951)

[40] A.E. Giannakopoulos, P-L. Larsson, and R. Vestergaard, Int. J. Solids Struct. 31, $2679(1994)$

[41] P-L. Larsson, A.E. Giannakopoulos, E. Soderlund, D.J. Rowcliffe, and R.

Vestergaard, Int. J. Solids Struct. 33, 221 (1996)

[42] M.M. Chandhri, Acta Mater. 46, 3047 (1998)

[43] http://www.gordonengland.co.uk/hardness/

[44] John H. Underwood, 'Residual-stress Measurement Using Surface Displacements Around an Indentation', Third SESA International Congress on Experimental Mechanics held in Los Angeles, CA on May 13-18, 1973

[45] Fleck, N A and Hutchinson, J W., 'A phenomenological theory for strain gradient plasticity.' J. Mech. Phys. Solids,41(12), pp 1825-1857, 1993.

[46] Fleck, N.A., Muller, G.M., Ashby, M.F., Hutchinson, J.W., 'Strain gradient plasticity: theory and experiment.' Acta Metall. Mater. 42, 475-487, 1994 [47] Stolken, J.S., Evans, A.G., 'A microbend test method for measuring the plasticity length scale.' Acta Mater. 46, 5109-5115. 1998 
[48] Lloyd, D.J., 'Particle reinforced aluminum and magnesium matrix composites.' Int. Mater. Rev. 39,1994

[49] Stelmashenko, N.A., Walls, A.G., Brown, L.M., Milman, Y.V., 'Microindentations on W and Mo oriented single crystals: an STM study.' Acta Metall. Mater. 41, 28552865,1993

[50] Ma, Q., Clarke, D.R., 'Size dependent hardness of silver single crystals.' J. Mater.

Res. 10, 853-863, 1996

[51] McElhaney, K.W., Vlassak, J.J., Nix, W.D., 'Determination of indenter tip geometry and indentation contact area for depth-sensing indentation experiments.' J. Mater. Res. 13, 1300-1306, 1998

[52] T.A. Laursen and J.C. Simo, 'A study of the mechanics of microindentation using finite elements.' J. Mater. Res. 7, 618-626, 1992

[53] E.R. Kral, K. Komvopoulos and D.B. Bogy, 'Elastic-Plastic Finite Element Analysis of Repeated Indentation of a Half-Space by a Rigid Sphere', Journal of Applied Mechanics, 60, 829-841, 1993

[54] Private Email discussions with Dr. F.M. Haggag, President and Chief Engineer, Advanced Technology Corp

[55] Jeong-Hoon Ahn, Dongil Kwon, 'Derivation of plastic stress-strain relationship from ball indentations: Examination of strain definition and pileup effect', J. Mater. Res., 16, $3170-3178,2001$

[56] R. Hill, F.R.S., B. Storakers and A.B. Zdunek, Proc. R. Soc. London, Ser, A. 423, 301,1989

[57] www.specialmetals.com 
[58] Chuanyu Feng, Evaluation of Material Mechanical Properties Using Depth Sensing Indentation and Combined Twyman-Green Interferometry and Moiré Interferometry, Proposal for PhD Dissertation, May 2004. 


\section{Ziheng Yao}

366 Newton Ave, Apt\#103 Morgantown, WV 26505

Phone: 304-685-3952(M) 304-293-2588(H) 304-293-3111 Ext. 2454(O)

Email: zihengyao@hotmail.com zyao@mix.wvu.edu

\section{Main Areas of Interest:}

Solid Mechanics (Mechanics of Materials, Theory of Elasticity and Plasticity, Continuum Mechanics, Viscoelasticity of Polymer, Composite Materials, Fracture Mechanics, Experimental Stress Analysis, Finite Element Method), CAD/CAE, Material Science, Designing and Manufacturing

\section{Education:}

$>$ Present, $\mathrm{PhD}$ (GPA: 4.0)

Department of Mechanical and Aerospace Engineering, West Virginia University

Dissertation: Development of an Indentation Method for Materials Surface Mechanical

Properties Measurement

$>$ 06/1999, M.S., Southwest Petroleum Institute (China)

Major: Mechanical Engineering

Thesis Title: Computer Aided Design and Analysis of Plate Drilling Bit

$>$ 06/1996, B.S., Southwest Petroleum Institute (China)

Major: Mechanical Engineering

\section{Experience:}

$>08 / 2004-12 / 2004$

Department of Mechanical and Aerospace Engineering, West Virginia University

Instructor of undergrad course MAE243 'Mechanics of Materials'

$>$ 07/2004-08/2004

Department of Mechanical and Aerospace Engineering, West Virginia University

Instructor of graduate course MAE640 'Continuum Mechanics'

$>$ 01/2002-06/2004

Department of Mechanical and Aerospace Engineering, West Virginia University

Graduate Research Assistant

- Determination of Material Mechanical Properties Using Micro-Indentation

- Development of Improved Materials for Continuous Steel Hot-dipping

- Time \& Frequency Viscoelastic Property of Commercial Polymers

- Magnetic levitate train's rail design and analysis

$>01 / 2001-12 / 2001$

Department of Mechanical and Aerospace Engineering, West Virginia University

Graduate Teaching Assistant

- MAE 345 Aerospace Structures, Instructor: Dr. Nithi Sivaneri

- MAE 242 Kinetics and Dynamics, Instructor: Dr. Victor H. Mucino

- MAE 456 CAD/Finite Element Analysis, Instructor: Dr. Victor H. Mucino

$>$ 07/1999-10/2000

Center for Well Completion Techniques, Southwest Petroleum Institute (China)

Research Assistant Professor

- Research area: Rock Mechanics, Well Stability

- Lecturing undergraduate course: Introduction to Computer Application

\section{Publications:}

Barbero, E. J., Julius, M. and Yao, Z. Time and Frequency Viscoelelastic Behavior of Commercial Polymers, CCC-2003, Calabria, Italy, September.

Ziheng Yao, Bruce Kang, 'Post-yielding Stress-Strain Evaluation Using Spherical Indentation: Bulk and Thin Film Materials' submitted to journal of 'Mechanics of Advanced Materials and Structures'. 


\section{Professional License:}

Engineer-In-Training (Registration Number: 29786)

\section{Computer Skill:}

Microsoft Windows; Office (Word, Excel, PowerPoint)

ANSYS, ABAQUS, FEMAP, IDEAS, Auto CAD, Pro/E

Visual Basic, MATLAB, MAPLE, ORIGIN

\section{Awards, Honors and Activities:}

07/2004, review paper entitled 'On anisotropic laminated plate bending problem: a comparison of two energy based solution approaches' submitted to journal 'Mechanics of Advanced Materials and Structures'.

05/2004, review paper entitled 'Three-D investigation of thick single lap bolted joint' submitted to journal of 'Experimental Mechanics'.

$12 / 2002$, attend industry symposium in Charleston, WV

03/2002, attend SAMPE regional conference in University of Maryland at college park, MD Member of Society for the Advancement of Material and Processing Engineering (SAMPE) 06 07/2000, oil field technical support in Changqing Oil Field for China's first underbalanced drilling well

06/1996, being awarded 'Excellent Graduate Student of Sichuan Province' 09 10/1994, attend curriculum practice training in Chengdu Petroleum Machinery General Plant 07/1994, attend 'excellent students summer camp' organized by Sichuan Province Educational Bureau 